\title{
SULFUR COATING OF UREA IN SHALLOW SPOUTED BEDS
}

by

MICHAEL MYUNG-SOO CHOI

B.Sc., University of Alberta, 1986

\author{
A DISSERTATION SUBMITTED IN PARTIAL FULFILLMENT OF \\ THE REQUIREMENTS FOR THE DEGREE OF \\ DOCTOR OF PHILOSOPHY \\ IN \\ THE FACULTY OF GRADUATE STUDIES \\ DEPARTMENT OF CHEMICAL ENGINEERING
}
We accept this thesis as conforming
to the required standard

THE UNIVERSITY OF BRITISH COLUMBIA

APRIL, 1993

(C) Michael M.S. Choi, 1993 
In presenting this thesis in partial fulfilment of the requirements for an advanced degree at the University of British Columbia, I agree that the Library shall make it freely' available for reference and study. I further agree that permission for extensive copying of this thesis for scholarly purposes may be granted by the head of my department or by his or her representatives. It is understood that copying or publication of this thesis for financial gain shall not be allowed without my written permission.

Department of Chemical Enginering

The University of British Columbia Vancouver, Canada

Date Apri) 20,1993 


\begin{abstract}
Sulfur coated urea (SCU) is an effective and economical slow-release nitrogen fertilizer, and its production in a spouted bed was investigated. SCU was produced by batch and continuous operations. Higher quality products were typically produced by the batch process, but at significantly lower production rates than the continuous process. In order to understand such operations, mathematical models describing the coating process were developed and verified through experiments.
\end{abstract}

The production of SCU was studied in shallow spouted beds fitted with a pneumatic molten sulfur spray nozzle located at the cone inlet. Bed hydrodynamics, coating mechanism, particle coating distribution and product quality were examined under the following conditions: Bed diameter of cylindrical section -0.24 and $0.45 \mathrm{~m}$; bed height -0.11 to 0.63 $\mathrm{m}$; included cone angle $-60^{\circ}$; particle diameter -2.1 to $2.8 \mathrm{~mm}$; particle density 930 to $1490 \mathrm{~kg} / \mathrm{m}^{3}$; main spouting air $\leq 37 \mathrm{~L}($ actual)/s; atomizing air $\leq 0.87 \mathrm{~L}$ (actual)/s; urea feed rate -7.6 to $20 \mathrm{~g} / \mathrm{s}$; sulfur injection rate -2.1 to $6.1 \mathrm{~g} / \mathrm{s}$; orifice diameter -21 to 35 $\mathrm{mm}$; bed temperature -18 to $70^{\circ} \mathrm{C}$; sulfur content $<60 \%$. The temperatures of atomizing air and molten sulfur were fixed for all runs at approximately 160 and $150{ }^{\circ} \mathrm{C}$, respectively. The coating process was successfully modeled using mass and momentum balance equations, inertial sulfur droplet deposition as the dominant coating mechanism, and Monte Carlo simulations.

The hydrodynamic model was based on the one-dimensional mass and momentum balances suggested by Lefroy and Davidson (1969) for gas and particle motion in the spout, the axial pressure correlation given by Morgan and Littman (1980), and the vector form of the Ergun (1952) equation for gas motion in the annulus. The effect of atomizing air entering through the spray nozzle was successfully incorporated into the model by considering the total momentum flux into the bed. Conical beds were found to behave similar to 
conical-cylindrical beds having a column diameter of $80 \%$ of the maximum conical bed diameter.

The dominant coating mechanism was deduced from the bed hydrodynamics and spray drop sizes produced by the pneumatic atomizing nozzle (type: internal mixing; Fluid Cap \# 40100; Air Cap \# 1401110; manufactured by Spraying System Co.). The drop sizes were found to range from approximately 6 to $50 \mu \mathrm{m}$ dia. The atomizing air flow rate did not affect the drop size distribution significantly under the conditions used in the present study. For the drop sizes produced and the hydrodynamic conditions prevailing in the spouted bed, inertial deposition was found to be the dominant mechanism for coating the bed particles.

On the basis of the bed hydrodynamics and the coating mechanism, the particle coating distributions were calculated utilizing the Monte Carlo method, and the quality of SCU particles was estimated from the coating distributions. The simulation results, which were in good agreement with the experimental data, imply that the product quality improved with increasing bed diameter, spouting and atomizing air flow rates, and that it decreased with increasing urea feed rates. Some improvement in product quality was also observed after changing the urea feed location and reducing the spray angle.

The model results also indicated that products with widely varying quality can be produced in a series of spouted beds at high production rates. This implies that the spouted bed is an effective and practical coating unit for producing SCU. 


\section{Table of Contents}

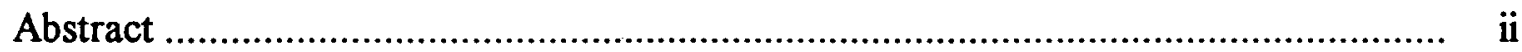

Table of Contents ................................................................................................. iv

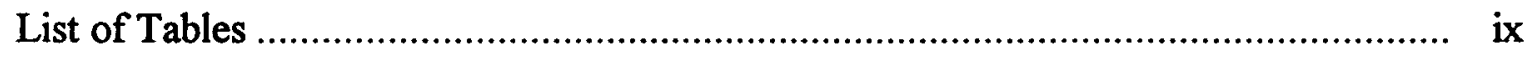

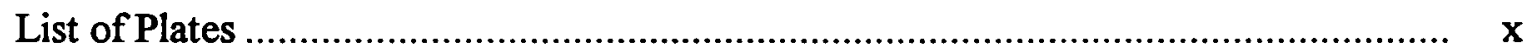

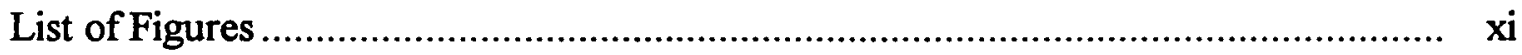

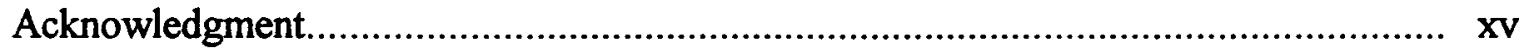

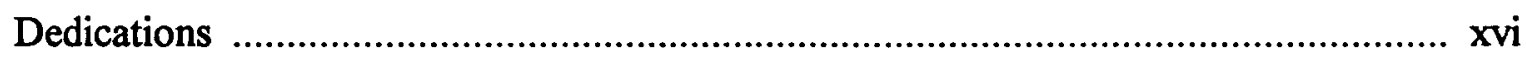

Chapter 1. Introduction ......................................................................... 1

1.1. The UBC Spouted Bed Process................................................................ 2

1.2. Objectives of Present Study................................................................. 5

1.2.1. Bed Hydrodynamics................................................................... 6

1.2.2. Coating Mechanisms................................................................ 6

1.2.3. Overall Coating Performance ...................................................... 6

1.2.4. Benefits of the Study .............................................................. 7

Chapter 2. Literature Review ................................................................... 8

2.1. UBC Process ........................................................................................ 8

2.1.1. Product Quality ........................................................................ 9

2.1.2. Effect of Bed Temperature on Product Quality ......................... 9

2.1.3. Effect of Sulfur Injection Rate on Product Quality .................... 11

2.1.4. Effect of Atomizing Air on Product Quality ............................... 11

2.1.5. Effect of Bed Depth on Product Quality.................................... 12

2.1.6. Effect of Spouting Air Flow Rate on Product Quality ................ 13

2.1.7. Effect of Chemical Additives on Product Quality ....................... 13

2.2. Models of Spouted Bed Coating Process .................................................. 13

2.3. Models and Correlations for Spouted Bed Hydrodynamics ...................... 16

2.3.1. Minimum Spouting Velocity ................................................ 16 
2.3.2. Solids Circulation and Bed Hydrodynamics.

2.3.3. Spout Diameter ............................................................... 18

2.3.4. Pressure Profile in Annulus ...................................................... 19

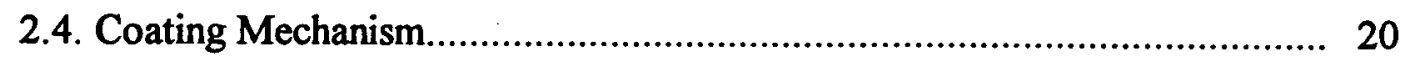

2.5. Monte Carlo Method............................................................................ 21

Chapter 3. Experimental Materials, Apparatus and Procedures ........................ 23

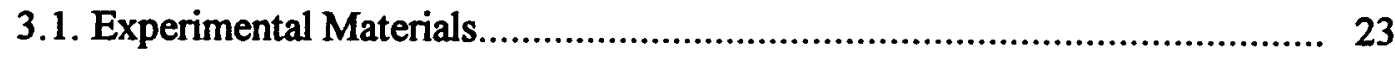

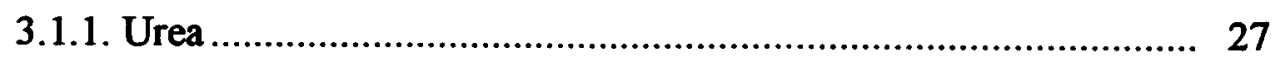

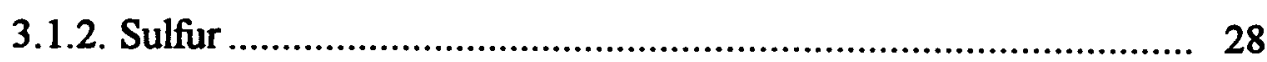

3.1.3. Particles Used in Hydrodynamics Study .................................. 29

3.2. Main Coating Apparatus ….............................................................. 30

3.2.1. Spouted Bed......................................................................... 32

3.2.2. Sulfur Supply System.......................................................... 34

3.2.2.1. Sulfur Melter............................................................. 34

3.2.2.2. Sulfur Filter................................................................ 35

3.2.2.3. Sulfur Rotameter..................................................... 35

3.2.2.4. Sulfur Line ............................................................ 36

3.2.2.5. Nitrogen Supply ................................................. 37

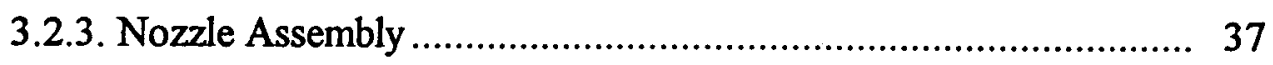

3.2.4. Urea Feeding Device .......................................................... 37

3.2.5. Product Withdrawal Device .................................................. 39

3.2.6. Product Collector ..................................................................... 39

3.2.7. Dust Collector ..................................................................... 39

3.2.8. Air, Steam, and Water Supplies .......................................... 40

3.3. Apparatus for Hydrodynamics Study ................................................... 40

3.4. Apparatus for Spray Study ................................................................ 41

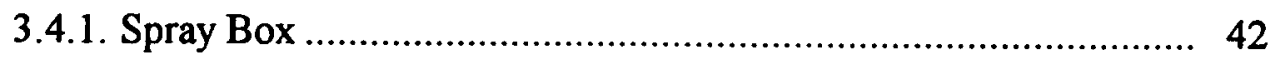

3.4.2. Spray Sampler ……....................................................... 43

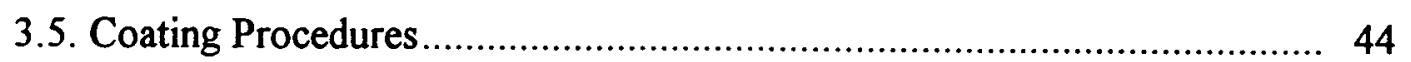

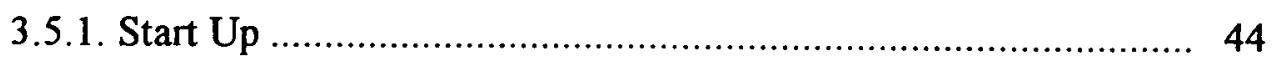


3.5.2. Coating

3.5.3. Shut Down and Clean-up...................................................... 46

3.6. Procedures for Hydrodynamics Study ................................................... 46

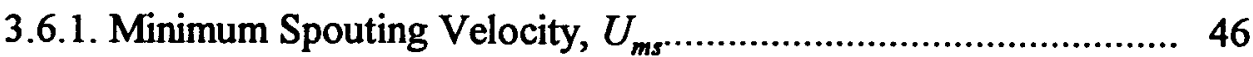

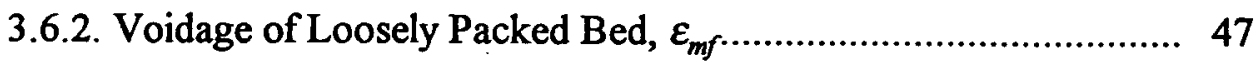

3.6.3. Mean Particle Diameter, $d_{p}$, and Sphericity, $\phi_{s} \ldots \ldots \ldots \ldots \ldots \ldots \ldots \ldots . . . . . . . . . . . . . .47$

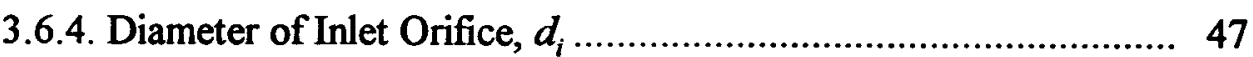

3.6.5. Static Pressure in Annulus ....................................................... 48

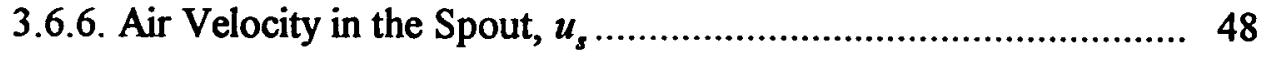

3.6.7. Radial Velocity Profile at the Base of the Bed ........................... 49

3.7. Procedures for Spray Studies ................................................................ 49

3.7.1. Operating Limits of Spray Nozzle............................................ 49

3.7.2. Spray Drop Size Measurements ............................................. 50

3.8. Product Quality Analysis ..................................................................... 50

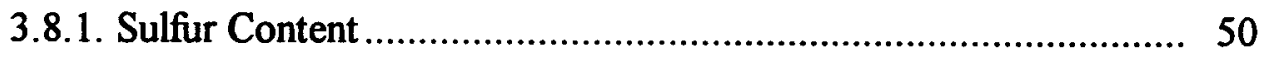

3.8.2. Particle Sulfur Content ......................................................... 51

3.8.3. Seven Day Dissolution Test ..................................................... 52

Chapter 4. Mathematical Models ...................................................................... 53

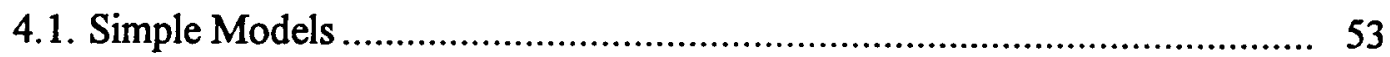

4.1.1. Model I: Residence Time Model ........................................... 53

4.1.2. Model II: Simple Spray Zone Model ........................................ 56

4.1.3. Model IIII: Variable Concentration Spray Zone Model............... 61

4.2. Model IV: Rigorous Model ................................................................ 62

4.2.1. Calculation of Models for Solids Circulation Rate and Bed Hydrodynamics................................................................... 63

4.2.2. Determination of Coating Mechanism and Concentration Profile.

4.2.3. Calculation of Coating Distribution Using the Monte Carlo Method. 68

4.2.3.1. Limitation of Analytical Model ..................................... 68

4.2.3.2. Monte Carlo Procedure for Model III......................... 69 
4.2.3.3. Monte Carlo Procedure for Model IV. 70

4.2.3.3.1. Continuous Operation................................ 70

4.2.3.3.2. Batch Operation ....................................... 72

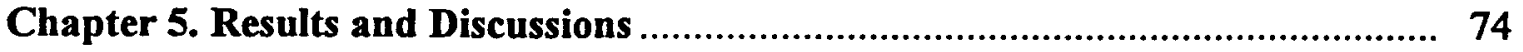

5.1. Bed Hydrodynamics ................................................................ 74

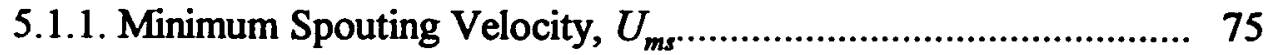

5.1.2. Pressure Profile in Annulus ............................................... 85

5.1.3. Velocity Profile in Annulus .................................................. 89

5.1.4. Velocity Profile in Spout...................................................... 89

5.1.5. Solids Movement.............................................................. 94

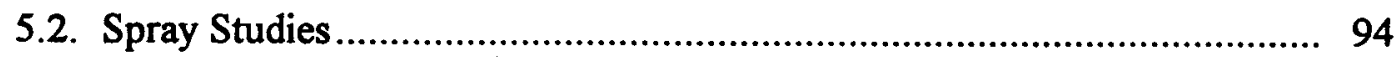

5.2.1. Operating Limits........................................................... 94

5.2.2. Spray Drop Size Distribution and Average Drop Size ............... 96

5.2.3. Coating Mechanism and Sulfur Spray Concentration.................. 102

5.3. Coating Distribution and Product Quality ........................................... 105

5.3.1. Coating Distribution .......................................................... 107

5.3.2. Product Quality ................................................................. 110

5.3.3. Effect of Operating and Model Variables on Coating Distribution

5.3.3.1. Effect of Operating Time....................................... 112

5.3.3.2. Effect of Sample Size ............................................ 115

5.3.3.2.1. Numerical Sampling................................... 115

5.3.3.2.2. Manual Sampling ..................................... 116

5.3.3.3. Effect of Spray Angle.......................................... 118

5.3.3.4. Effect of Feed Location............................................ 120

5.3.3.5. Effect of Beds-in-Series......................................... 121

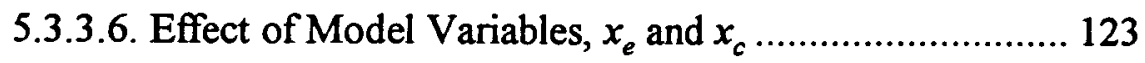

5.3.4. Sensitivity Analysis Using Model IV ..................................... 125

5.4. Commercial Implications ........................................................... 130

Chapter 6. Conclusions and Recommendations ............................................. 131

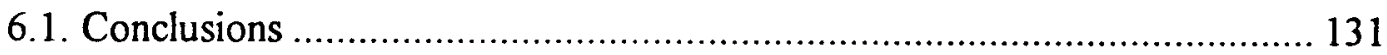




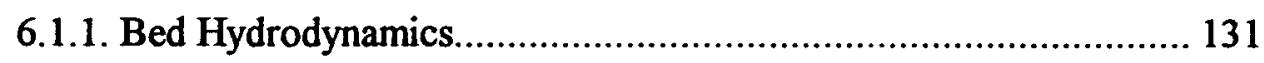

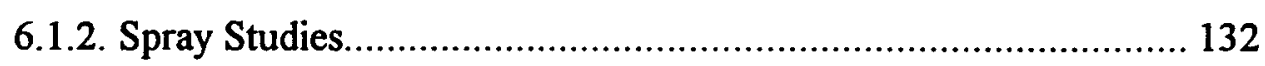

6.1.3. Coating Distribution and Product Quality.................................. 132

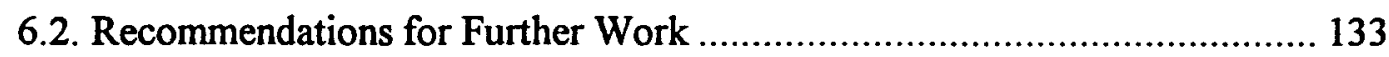

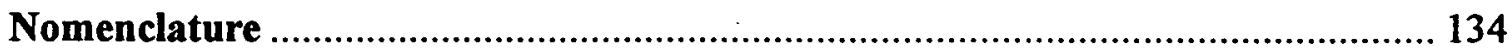

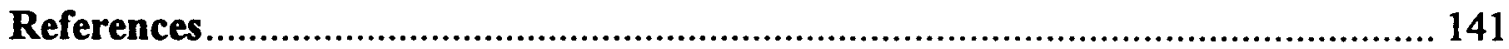

Appendix I: Supporting Derivations and Methodology ..................................... 149

I-1. Determination of Shutter Area .......................................................... 150

I-2. Model II Derivation for Forced Urea Feed.............................................. 152

I-3. Calculation Method for Vector Ergun Equation........................................ 154

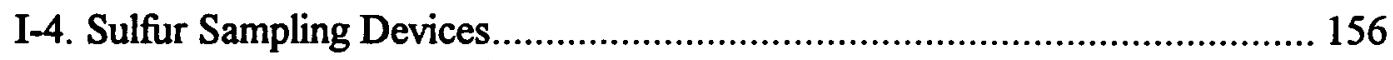

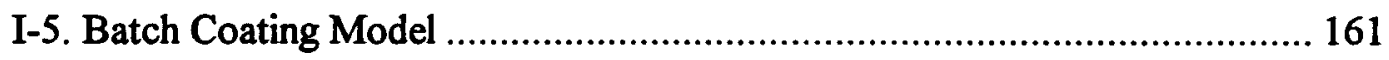

I-6. Minimum Spouting Velocity Predictions by Wan-Fyong et al. Equation ..... 163

Appendix II: Experimental Data and Calculated Results ....................................... 164

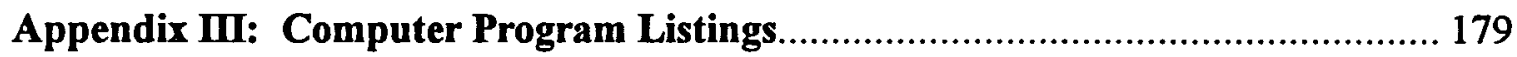

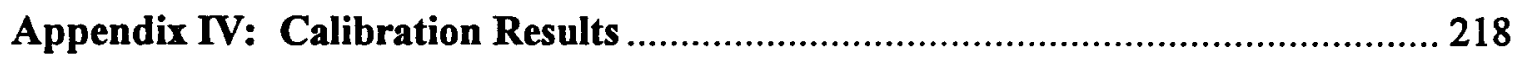




\section{List of Tables}

Table page

1.1 Major agronomic benefits associated with SCU usage (Tisdale et al., 1985) ........ 1

2.1 Range of operating conditions used in previous studies on the UBC process ........ 9

3.1 Selected physical properties of urea (Perry et al., 1984) .................................... 27

3.2 Selected chemical and physical properties of sulfur (Stauffer Chemical Co.) ......... 28

3.3 Properties of common sulfur allotropes (Donahue and Meyer, 1965; Dale and Ludwig, 1965) ............................................................................ 28

3.4 Physical properties of bed particles ....................................................... 30

3.5 Types and capacities of Brooks rotameters used in this work ........................... 36

4.1 Assumptions used in simple models ......................................................... 58

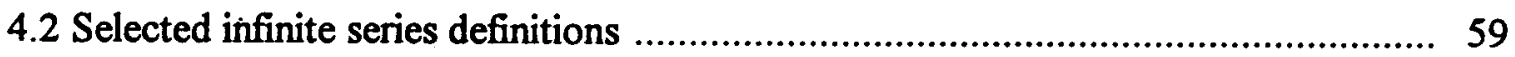

5.1 Operating ranges applicable to this work ................................................... 75

5.2 Operating conditions under which the air velocities in the spout and the pressure profiles in the annulus were determined ........................................... 76

5.3 Axial pressure profile near the spout-annulus interface .................................... 77

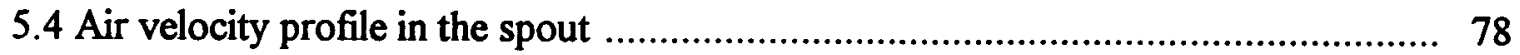

5.5 Fitted constants for the $\mathrm{Wu}$ et al. (1987) equation .......................................... 80

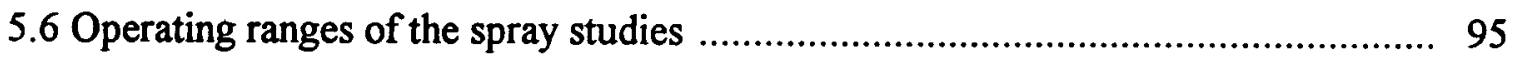

5.7 Results of drop size studies at various operating conditions ............................... 100

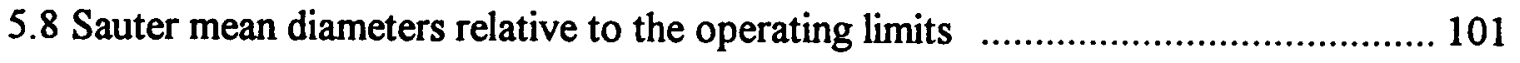

5.9 Mechanical collection efficiency (using correlations suggested by Clift et al., 1981) at selected operating conditions in the spout .................................. 102

5.10 Operating range applicable to coating distribution and product quality studies ..... 106

5.11 Operating conditions investigated for the coating study ............................... 106

5.12 Errors associated with sample sizes based on the Model II and III results ............ 116 


\section{List of Tables Continued}

Table

5.13 Effect of feed location (using Model II with $x_{c}=0.5, \bar{X}_{s}=0.25$ )

5.14 Range of model variables examined for sensitivity analysis

5.15 Results of sensitivity analysis using Model IV $\left(\bar{X}_{s}=0.25, T=60^{\circ} \mathrm{C}\right)$ 128

I-1 Direct sulfur sampling 158

I-2 Hot tip: packed bed $\mathrm{S} \Rightarrow \mathrm{SO}_{2}$ converter 158

I-3 Hot tip: plate $\mathrm{S} \Rightarrow \mathrm{SO}_{2}$ converter 159

I-4 Hot tip: laser beam $\mathrm{S} \Rightarrow \mathrm{SO}_{2}$ converter 159

I-5 Hot tip: three tube hot wire $\mathrm{S} \Rightarrow \mathrm{SO}_{2}$ converter 160

I-6 Hot tip: two tube hot wire $\mathrm{S} \Rightarrow \mathrm{SO}_{2}$ converter 160

II-1 Minimum spouting velocity data 165

II-2 Spray drop size distribution 174

II-3 Measured sulfur content of individual particles for selected continuous runs 176

II-4 Predicted sulfur content of individual particles for selected continuous runs 177

III-5 Measured sulfur content of individual particles for batch runs shown in Figures 4.3 and 5.25 178

IV-1 Calibration equations for flowmeters and refractometer 219

\section{List of Plates}

$\underline{\text { Plate }}$

page

3.1 Urea

3.2 Sulfur coated urea produced by batch process

3.3 Sulfur coated urea produced by continuous process

3.4 Polyformaldehyde 25

3.5 Polyethylene 26

3.6 Polystyrene 26 


\section{List of Figures}

Figure

page

1.1 Schematic diagram of UBC spouted bed coating unit for producing sulfur coated urea (heavy arrows indicate direction of solids flow) ........................ 3

1.2 Sulfur content of CIL and UBC products (Tsai, 1986) ................................... 5

2.1 Effect of bed temperature on product quality. (The scatter results from the effects of other variables) ................................................................. 10

2.2 Effect of atomizing air flow rate on dissolution rate normalized for the sulfur flow rate (Weiss, 1981) ............................................................ 12

3.1 Viscosity of sulfur at low temperature range (Freeport Sulfur Co., 1954) ............. 29

3.2 Simplified flowsheet of UBC spouted bed facility ........................................ 31

3.3 Sectional view of spouted bed column .................................................... 32

3.4 Shutter assembly (dimensions are given in $\mathrm{mm}$ ) designed by Mathur, Meisen

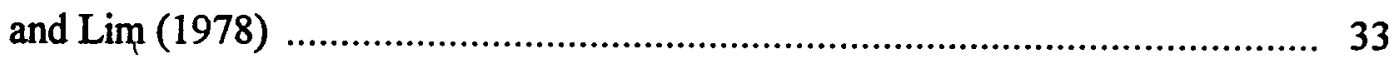

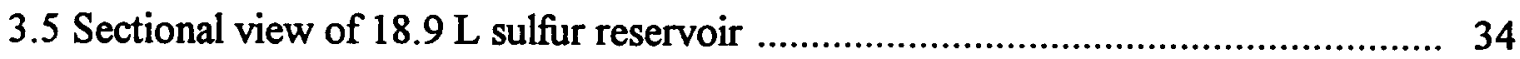

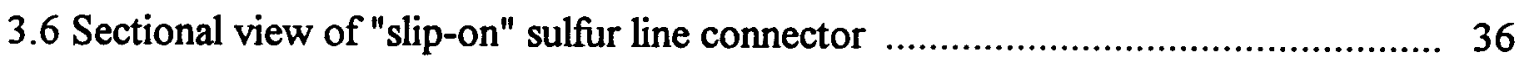

3.7 Sectional view of nozzle, perforated plate and steam chamber (all dimensions in $\mathrm{mm}$; designed by Meisen, Lee and Le, 1986) ....................................... 38

3.8 Modifications to the top of the spouted bed (i.e., see Figure 3.3) for hydrodynamics study

3.9 Schematic diagram of static pressure probe

3.10 Schematic diagram of S-type pitot tube (1/8" tubes and 3/8" tube are held in place with silver solder)

3.11 Spray box assembly 43

3.12 Simplified drawing of the rotating sulfur droplet sampler 44

3.13 Sectional view of atomizing nozzle and inlet air assembly (not drawn to scale) ... 48

4.1 Model I - perfectly mixed vessel 54 


\section{List of Figures Contimued}

Figure page

4.2 Prediction of Model I using different numbers of continuously stirred tanks in

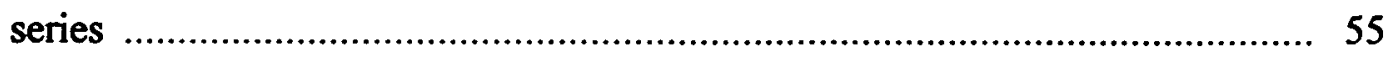

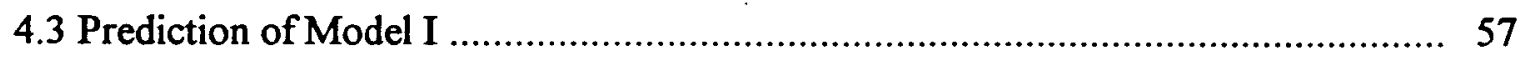

4.4 System described by Model II ................................................................... 58

4.5 Sectional view of the lower spout ............................................................... 66

4.6 Concentration balance on coating material in the spout .....................................67

5.1 Comparison between experimental and predicted $U_{m s}$ values ............................... 79

5.2 Radial velocity profile $10 \mathrm{~mm}$ above the shutter in an empty bed $\left(Q_{s}=\mathbf{2 7 . 9}\right.$

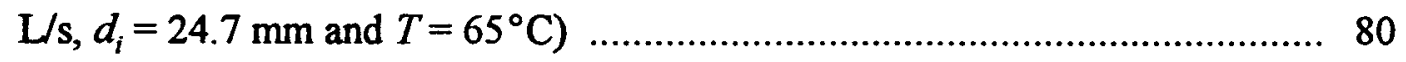

5.3 Effect of atomizing air on minimum spouting velocity (minimum spouting velocity is based only on the main spouting air flow rate; $D=0.45 \mathrm{~m}, H=$ $0.31 \mathrm{~m}, d_{i}=35 \mathrm{~mm}$ ) 82

5.4 Momentum flow of air into the spouted bed (dashed and solid lines represent $0.24 \mathrm{~m}$ and $0.45 \mathrm{~m}$ dia. beds, respectively) ...............................................

5.5 Effect of bed height on minimum spouting velocity, $U_{m s .}$ (Cone-cylinder junctions are denoted by dashed lines; solid lines represent indicated relationships fitted to experimental data)

5.6 Comparison between experimental data and predictions from correlation based on the optimum diameter $D^{\prime}$

5.7 Pressure profile in the annulus (conical bed, Run H22) 86

5.8 Axial pressure profile in the annulus near the spout-annulus interface (conicalcylindrical bed, Run H1) 86

5.9 Axial pressure profile in the annulus (conical bed, Run H22, column diameter used in calculation)

5.10 Axial pressure profile in the annulus (conical bed, Run $\mathrm{H} 22, D^{\prime}$ used in calculation)

5.11 Axial pressure profile in the annulus $(H=0.53$, Run $\mathrm{H} 17)$ 88 


\section{List of Figures Contimued}

Figure page

5.12 Comparison between measured and predicted pressure profile in the annulus ...... 88

5.13 Effect of atomizing air on axial air velocity profile in the spout $\left(Q_{s}\right.$ is fixed at approximately $32 \mathrm{~L} / \mathrm{s}$ in all runs) ..................................................... 90

5.14 Air velocities in the spout determined experimentally and from Model IV hydrodynamics (Run H1) ............................................................... 91

5.15 Air velocities in the spout determined experimentally and from Model IV hydrodynamics and Equation (5.5) (Run H22) ........................................ 91

5.16 Operating limits for atomization of sulfur .................................................. 96

5.17 Number distribution and predictions using log-normal equation (Run Sla) ......... 97

$5.18 \mathrm{Log}$-normal representation of drop size distribution (Run S1a) ........................ 98

5.19 Nukiyama-Tanasawa representation of drop size distribution (Run S1a) ............. 98

5.20 Predictions of Sauter mean diameter ........................................................ 101

5.21 Sulfur concentration profile for various spray angles ...................................... 104

5.22 Sulfur concentration profile for various coating runs $\left(\phi=20^{\circ}\right)$.......................... 104

5.23 Comparison between measured and predicted coating distributions for Run

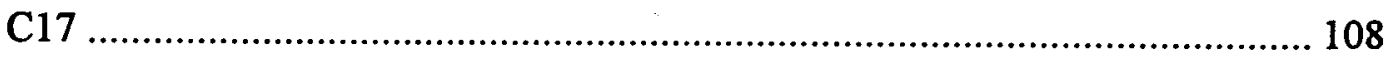

5.24 Comparison between measured and predicted coating distributions for Run C38 108

5.25 Coating distributions of batch products 109

5.26 Relationship between 7-day dissolution and sulfur content for batch products ..... 111

5.27 Comparison between measured and predicted product quality .......................... 112

5.28 Effect of operating period on coating distribution ......................................... 114

5.29 Effect of sample size on coating distribution (Models II and III) ....................... 117

5.30 Effect of sample size on coating distribution (Model IV) ............................... 117

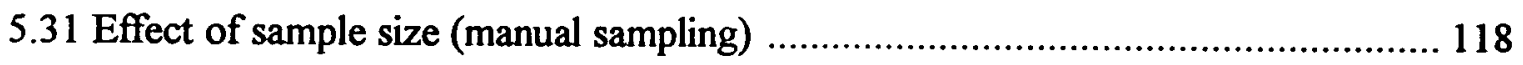

5.32 Effect of spray angle on coating distribution (results from Model IV) ................. 119 


\section{List of Figures Continued}

Figure

page

5.33 Effect of the spray concentration on coating distribution (results were obtained from Model III using Equation (4.19)) .................................................. 119

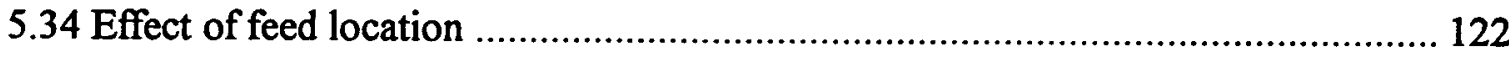

5.35 Product coating distributions for beds-in-series (from Model IV) ....................... 122

5.36 Sensitivity analysis of $x_{e}$ on coating distribution using Model II $\left(x_{c}=0.6\right) \ldots \ldots \ldots . . .124$

5.37 Sensitivity analysis of $x_{c}$ on coating distribution using Model II $\left(x_{e}=0.2\right) \ldots \ldots \ldots . .124$

5.38 Model predictions of product quality .............................................................. 126

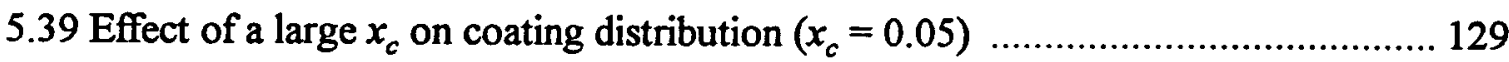

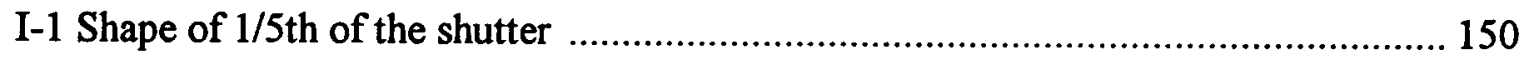

I-2 Shape of a section of fully open shutter with the same base length $(t)$ shown in

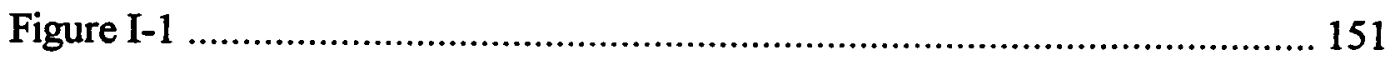

I-3 Simplified flow sheet of a forced feed system ................................................. 152

I-4 Minimum spouting velocity predictions using Wan-Fyong et al. (1969)

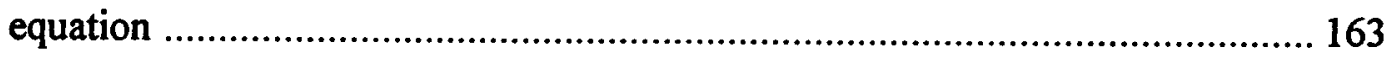

IV-1 Calibration curve for the lower capacity spouting air rotameter ........................... 220

IV-2 Calibration curve for the higher capacity spouting air rotameter ......................... 221

IV-3 Calibration curve for the atomizing air rotameter ............................................. 222

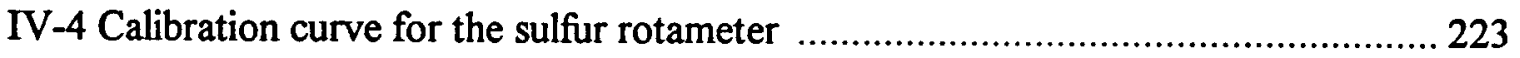

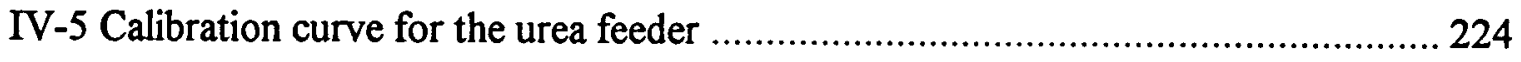

IV-6 Predictions using the calibrated value of $C_{o}$ for the static-pitot tube .................... 225

IV-7 Predictions using the calibrated value of $C_{o}$ for the S-type pitot tube .................. 226

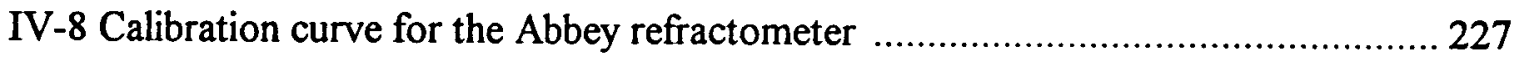




\section{Acknowledgement}

I wish to express my sincere gratitude to Dr. A. Meisen for his helpful suggestions and guidance which played a crucial role in the completion of this study.

I wish to thank my committee members for their willing assistance and suggestion - especially Dr. I. Gartshore for his assistance with designs of fluid velocity measurement devices.

I also wish to extend my sincere appreciation to the following individuals and departments for their assistance:

- Mr. Van Quan Le and Mr. Victor Lee for their assistance with the operation of the spouted bed coating unit,

- The Workshop, Stores, faculty and staff of Chemical Engineering for their assistance,

- The Department of Metals and Materials Engineering for their assistance with the use of image analyzer.

Finally, I wish to thank my family for their continued encouragement, patience and understanding. I am especially grateful to my father for encouraging me to pursue a higher degree and for understanding my inability to fulfill my role as a son during the course of this project. I am also indebted to my wife for single-handedly taking care of our daughter on top of working to make ends meet. 
Dedicated to ney father.

mes wefe gean.

and my daughter Ashleigh... 


\section{Chapter 1. Introduction}

Sulfur coated urea (SCU) has been proven as an effective and economical slow release nitrogen fertilizer. SCU is produced by applying a light coating of water resistant sulfur on urea granules. In soil, the sulfur is slowly degraded by microorganisms and the urea is thereby exposed. For this reason SCU is classified as a slow release nitrogen (SRN) fertilizer. Previous studies (Davis, 1973; Waddington and Duich, 1976; Allen et al., 1971) showed that SCU is at least as effective as other SRN fertilizers or repeated applications of uncoated nitrogen fertilizers. Moreover, SCU is the least expensive SRN fertilizer currently on the market, and has the highest nitrogen content. Other agronomic benefits of using SCU are summarized in Table 1.1.

The major disadvantages of SCU, are that the sulfur increases the soil acidity and lowers the nitrogen content of the fertilizer. Although lime application can mitigate the acidifying effect and greater fertilizer dosage can make up the necessary nitrogen requirement, both remedies add to the total cost. Therefore, it is important to reduce the sulfur content of SCU without significantly lowering the quality of SCU as a slow release nitrogen fertilizer.

Table 1.1: Major agronomic benefits associated with SCU usage (Tisdale et al., 1985).

\begin{tabular}{|c|c|}
\hline Benefits as a slow-release nitrogen (SRN) fertilizer: & $\begin{array}{l}\text { Benefits of sulfur }{ }^{*} \text { (for sulfur } \\
\text { deficient soils): }\end{array}$ \\
\hline - Increases efficiency of nitrogen use by the crop & \multirow{2}{*}{$\begin{array}{l}\text { - Increases intensity of grass } \\
\text { color } \\
\end{array}$} \\
\hline - Reduces toxicity to the crop & \\
\hline - Reduces application cost & \multirow{3}{*}{$\begin{array}{c}\text { - Enhances crop growth when } \\
\text { applied with nitrogen }\end{array}$} \\
\hline $\begin{array}{l}\text { - Decreases fertilizer run-off which pollutes local } \\
\text { waters }\end{array}$ & \\
\hline - Increases fertilizer storage life & \\
\hline
\end{tabular}


In order to produce high quality SCU an understanding of the production process is important. Two processes have been studied for manufacturing SCU: the Tennessee Valley Authority (TVA) rotary drum process and the UBC spouted bed process. The TVA process and its development were summarized by Tsai (1986) and will not be repeated here. The UBC process is the objective of this study and its description and developments are summarized in the following section.

\subsection{The UBC Spouted Bed Process}

Development of the spouted bed sulfur coating process started in 1975 by Meisen and Mathur. The equipment consisted mainly of a cylindrical vessel with a conical base filled with urea granules as shown in Figure 1.1. Air injected at the base of the apparatus forms a jet (spout) carrying particles entrained from the dense surrounding region (annulus). The particles are carried upwards until they reach the top of the bed (fountain) whence they fall back into the annulus. A cyclic pattern of particle movement is thereby established.

Coating is accomplished by spraying molten sulfur into the bottom of the bed coaxially with the spouting air. Each time a urea granule passes through the spray zone, it acquires a layer of sulfur which solidifies (if the bed is properly operated) by the time the particle reaches the top of the bed. Repeated passages through the spray zone build up the coat and reduce coat imperfections.

The SCU quality, expressed in terms of the 7-day dissolution $\left(D_{25}\right)^{1}$ value, was found to depend principally on bed temperature and sulfur flow rate. Initial experiments and dissolution tests demonstrated poor reproducibility. Operational problems including nozzle

\footnotetext{
${ }^{1} D_{25}$ denotes the percentage of urea which dissolves when $50 \mathrm{~g}$ of sample containing $25 \mathrm{wt} \%$ sulfur are placed in $250 \mathrm{~mL}$ of water at $37.8^{\circ} \mathrm{C}$ for 7 days.
} 


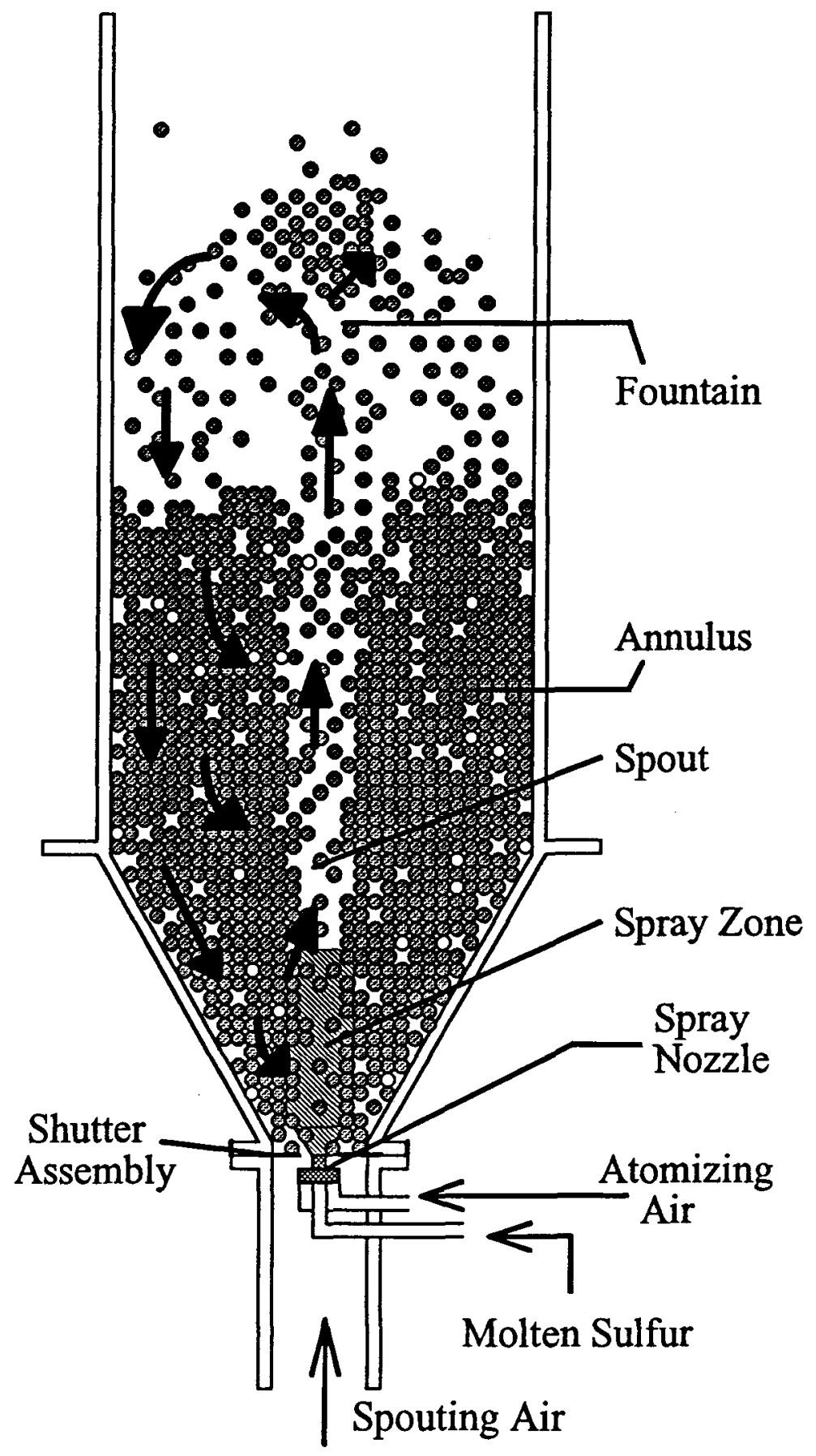

Figure 1.1: Schematic diagram of UBC spouted bed coating unit for producing sulfur coated urea (heavy arrows indicate direction of solids flow). 
plugging and sulfur handling difficulties led to further studies by Meisen and co-workers (Zee, 1977 and Lim, 1978).

Successful batch-wise coating was achieved by Weiss and Meisen $(1981,1983)$. The product quality $\left(D_{25}\right)$ was comparable to that of the CIL product made by the TVA process, and was found to depend on the sulfur droplet size, the spray distribution and the nature of the urea surface.

Some operational aspects of the continuous spouted bed process were examined by Meisen and Tsai (1986). In their initial study, they found the product from the UBC process gave higher $D_{25}$ values (i.e., had lower quality) than those of the CIL product. They suspected that the higher $D_{25}$ values of the UBC product resulted from a significant fraction of uncoated particles in the product. This is supported by Figure 1.2 which is a plot of the percentage of particles which contain less than a certain sulfur content (denoted by Probability \%) as a function of sulfur content. The cumulative percentage is shown on a "probability scale". The latter implies that the plot would be a straight line if the sulfur content is normally distributed around the mean value. Figure 1.2 shows that $27 \%$ of the particles contained less than $10 \%$ sulfur while only $5 \%$ of the particles from the CIL product contained less than $10 \%$ sulfur. Meisen and Tsai suspected that these uncoated particles resulted from fresh urea particles bypassing the spray zone and leaving the spouted bed prematurely. By changing the feed location and operating variables such as the bed height and the flow rate of the spouting air, a product comparable to the $\mathrm{CI}$ product in terms of $D_{25}$ values was obtained.

Many experiments are generally required to determine the effects of operating variables on the product quality. Since the cost of the experiments is high, an alternative method is sought. One way to reduce the number of experiments is to develop a mathematical model describing the coating process. 


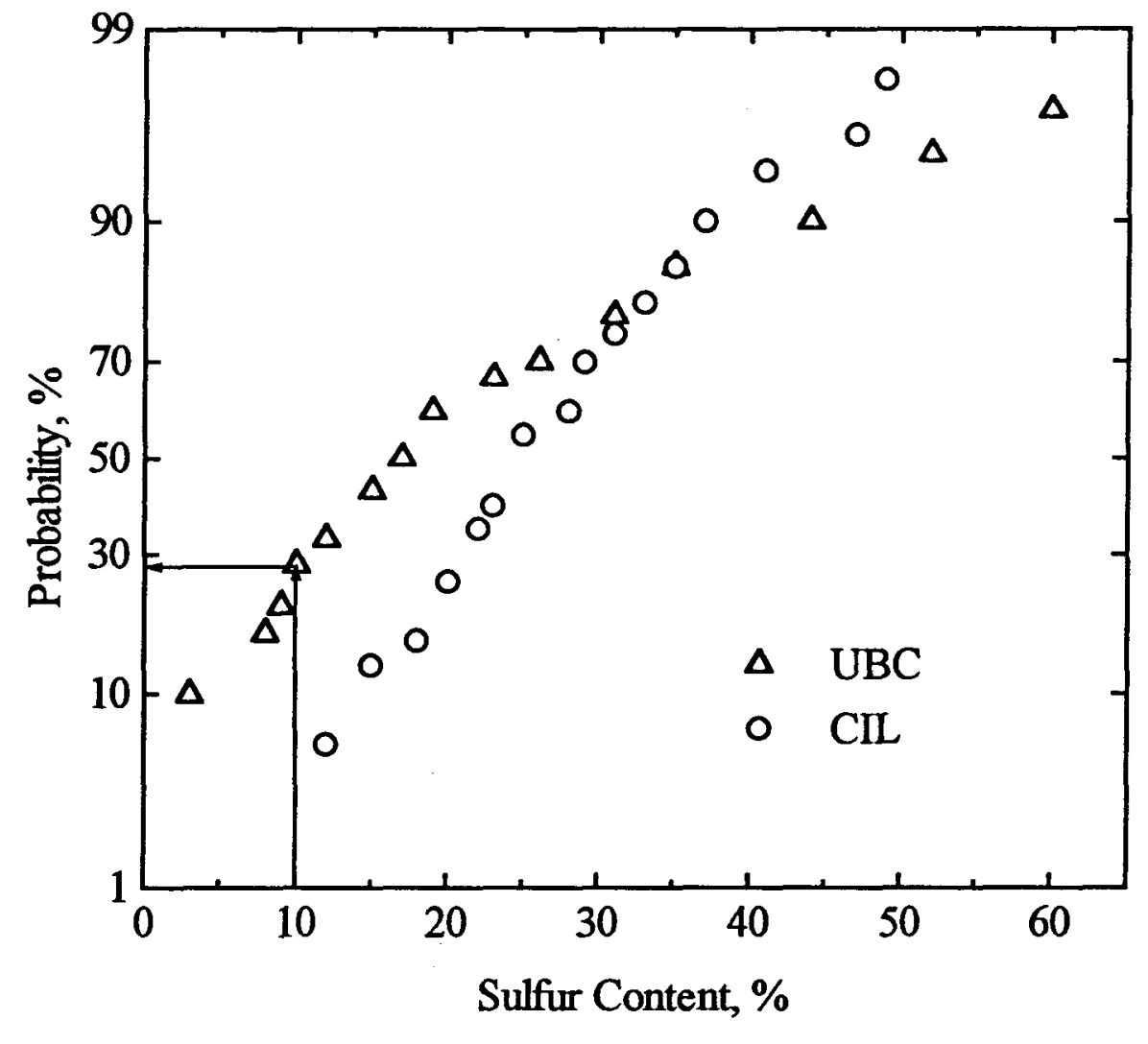

Figure 1.2: Sulfur content of $\mathrm{CIL}$ and UBC products (Tsai, 1986).

\subsection{Objectives of Present Study}

The main objective of the present study is to develop mathematical models for the spouted bed coating process for the production of sulfur coated urea. The models use probabilistic approaches and involve empirical and theoretical sub-models for the fluid and particle hydrodynamics and for the coating mechanism. Particular emphasis is placed on shallow beds (i.e., conical beds and beds extending just above the conical section) since they have low pressure drops and are therefore of greatest commercial interest. Other objectives of this study include the development and verification of the sub-models. The latter are presented in the following subsections. 


\subsubsection{Bed Hydrodynamics}

Although many hydrodynamic models have been reported for conventional spouted beds, the hydrodynamics of spouted beds configured for coating has not been addressed in the literature. In particular, the special geometry at the air inlet, atomizing air and coating agent make the coater behave differently from conventional beds. The objectives of the hydrodynamic study are to:

- determine the effects of atomizing air and bed geometry on the air velocities in the bed, including the minimum spouting velocity;

- develop mathematical models (or modify existing models) to describe the bed hydrodynamics in a spouted bed coater;

- verify the models by experimentally examining the fluid velocities in the spout and the pressure distribution in the annulus.

\subsubsection{Coating Mechanisms}

The coating mechanism governs the rate at which the sulfur droplets deposit onto urea particles and, ultimately, the spray concentration profile in the bed. The objectives of this part of the study are to determine the coating mechanism by experimentally analyzing the spray droplet size distribution.

\subsubsection{Overall Coating Performance}

An important objective of this work is the verification of the model predictions on the coating performance of the bed. Once the model is developed, the following verifications are conducted:

- the coating distribution of particles is determined for selected experiments;

- the coating distribution is correlated with the product quality expressed in terms of $D_{25}$ values. 


\subsubsection{Benefits of the Study}

Use of a mathematical model may be an inexpensive and fast alternative to conducting experiments to determine the optimum operating conditions. The model may also be useful in designing commercial plants because the product quality information is readily predictable for any size and number of beds the plant may require. The commercial implications based on model predictions are presented in Chapter 5 .

A better understanding of the following areas is also achieved as a result of this study:

- the effectiveness of coating models of various complexities is identified;

- the bed hydrodynamics of a spouted bed configured for coating are elucidated;

- the sulfur atomization and urea-sulfur contact mechanisms are characterized.

It should be noted that the physical bonding of sulfur on urea, the related effect of temperature and the influence of chemical additives are not examined in this work. The effect of bed temperature on product quality is investigated only through its impact on bed hydrodynamics. 


\section{Chapter 2. \\ Literature Review}

\subsection{UBC Process}

Although the spouted bed coating process has been studied in the past (Singiser et al., 1966; Umaki and Mathur, 1976), the effects of individual operating variables on product quality were not investigated until Meisen and Mathur commenced their research in 1975 . The results obtained by Meisen and co-workers since 1975 are reviewed in this section.

The batch-wise ${ }^{2}$ production of sulfur coated urea was studied by Meisen and co-workers - Mathur (1978), Zee (1977), Lim (1978), and Weiss (1981, 1983), and the continuous ${ }^{3}$ production was studied by Meisen and Tsai (1986). Most of the earlier work was devoted to improving the operational aspects of the process. (Detailed description of equipment modifications are provided by Weiss (1981) and by Tsai (1986)). With an improved coating facility, Lim was able to quantitatively explain the effects of the principal operating variables on the product quality for the batch process. Weiss followed up with more equipment modifications and more extensive studies of the batch process. Tsai extended the investigation to continuous operation. The operating conditions studied are summarized in Table 2.1 .

The principal operating variables that were found to affect the product quality were bed temperature $\left(T_{b}\right)$, sulfur injection rate $\left(W_{s}\right)$, atomizing air flow rate $\left(Q_{a}\right)$, bed depth $(H)$,

\footnotetext{
2 The "batch process" refers to a process where a batch of urea is placed in the spouted bed, coated and discharged.

${ }^{3}$ The "continuous process" refers to the process where urea is continously fed to the the bed and coated product (SCU) is continuously discharged from the bed.
} 
Table 2.1: Range of operating conditions used in previous studies on the UBC process. (Fixed variables: Column diameter $=0.15 \mathrm{~m}$, cone angle $=60^{\circ}$, nozzle type $=$ "internal-mixing" (supplied by Spraying Systems Co., fluid cap \#2050, air cap \#67147)).

\begin{tabular}{|l|c|c|c|c|c|c|}
\hline Authors & $H, \mathrm{~m}$ & $T_{b},{ }^{\circ} \mathrm{C}$ & $Q_{s} \mathrm{~m}^{3} / \mathrm{min}$ & $Q_{a}, \mathrm{~m}^{3} / \mathrm{h}$ & $W_{s}, g / m i n$ & $d_{i}, \mathrm{~mm}$ \\
\hline Lim, 1978 & $\sim 0.41$ & $37-80$ & $0.38-1.28$ & $0.39-0.83$ & $54-86$ & 16.1 \\
\hline Weiss, 1981 & $0.27-0.54$ & $48-86$ & $0.89-1.44$ & $0.4-0.79$ & $34-260$ & $\mathrm{n} / \mathrm{a}$ \\
\hline Tsai, 1986 & $0.15 \& 0.24$ & $50-87$ & $0.6-1.1$ & $0.35-0.65$ & $22-76$ & $\mathrm{n} / \mathrm{a}$ \\
\hline
\end{tabular}

spouting air flow rate $\left(Q_{s}\right)$ and chemical additives. A summary and review of the findings and explanations by Lim, Weiss, and Tsai are provided in the following sub-sections.

\subsubsection{Product Quality}

The quality of sulfur coated urea may be evaluated by laboratory or field tests. The seven day dissolution test was developed by TVA to measure the product quality in the laboratory and the dissolution value $\left(D_{25}\right)$ obtained from this test has been adopted as the standard measure of the product quality by Meisen and co-workers.

Tsai found that products could have the same $D_{25}$ value even though their instantaneous dissolution rates were very different. In particular, high initial dissolution rates were typically observed with products from the continuous process.

\subsubsection{Effect of Bed Temperature on Product Quality}

Previous researchers of the TVA process (Shirley and Meline, 1975) and the UBC process found that the bed temperature was the most important operating parameter affecting the product quality. For both processes, the optimum coating temperature was near $80^{\circ} \mathrm{C}$; however, the results (see Figure 2.1) for the UBC process show considerable scatter. The scatter was explained by the effects of other operating variables on $D_{25}$ (Meisen and Mathur, 1978; Weiss and Meisen, 1983). 


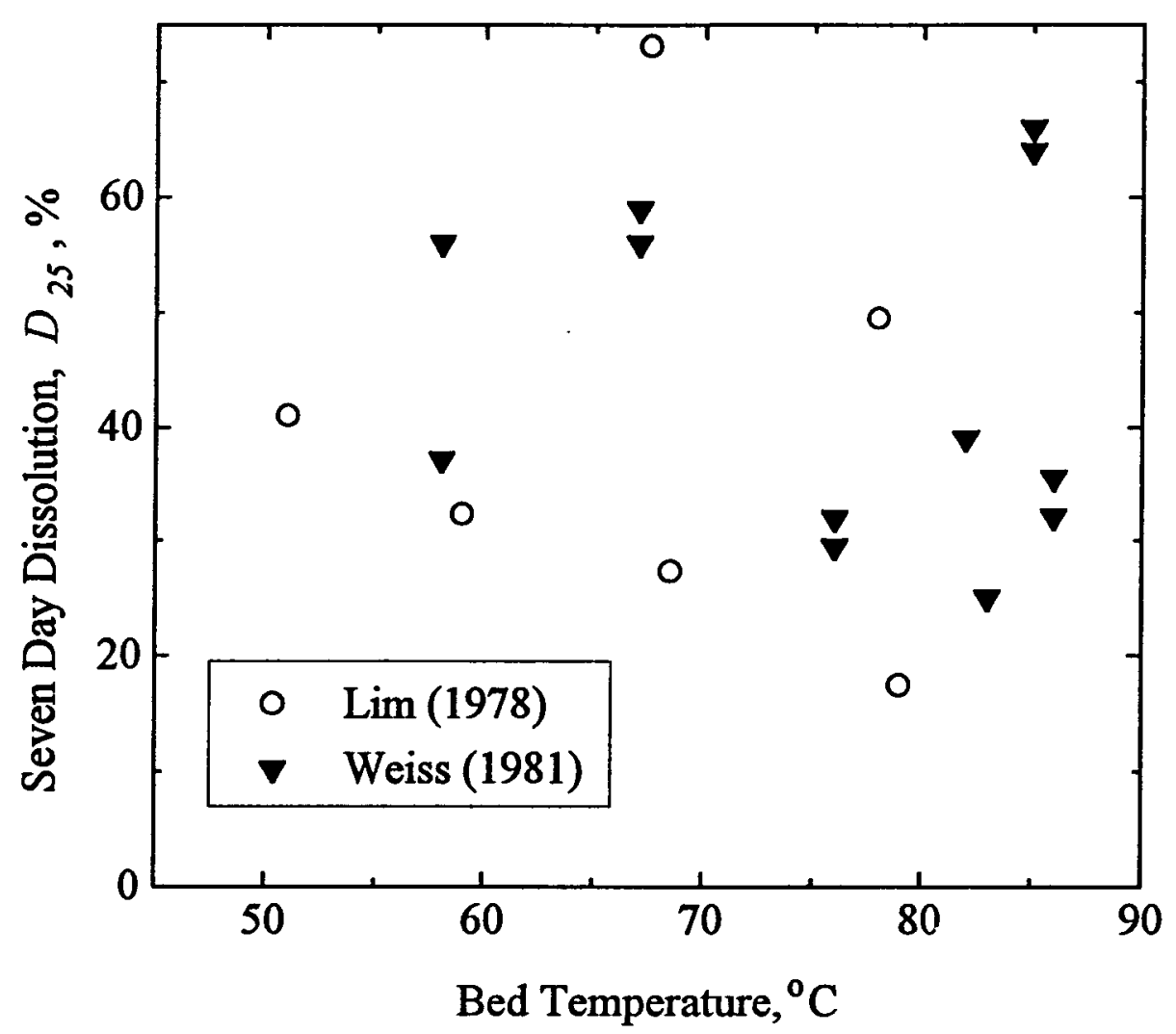

Figure 2.1: Effect of bed temperature on product quality. (The scatter results from the effects of other variables).

Upon examination of the coat surface under high magnification, Weiss was able to provide some explanations for the optimum coating temperature. For bed temperatures below 80 ${ }^{\circ} \mathrm{C}$, premature freezing of sulfur droplets before impingement onto the bed particles prevented the sulfur from spreading evenly on the urea surface. As a result, the surface appeared lumpy with gaps between the lumps, and the presence of these gaps enhanced the passage of water to the urea core thereby increasing the urea dissolution rate. Urea coated significantly above $80^{\circ} \mathrm{C}$ showed cracks in the coats. The cracks were thought to be caused by the contraction of sulfur as it changed from the monoclinic allotrope $\left(\mathrm{S}_{\beta}\right)$ of lower density to the orthorhombic form $\left(\mathrm{S}_{\alpha}\right)$ of higher density upon cooling. For bed tem- 
peratures below $80^{\circ} \mathrm{C}, \mathrm{S}_{\beta}$ was assumed to be absent from the coats and therefore major thermal contraction did not take place.

\subsubsection{Effect of Sulfur Injection Rate on Product Quality}

The experimental results obtained by Lim and Weiss suggest that the sulfur feed rate had a strong effect on the product quality. To determine the relationship between the sulfur injection rate and $D_{25}$, Weiss conducted several experiments by varying only the sulfur feed rate at a given bed temperature. The results showed the logarithm of $D_{25}$ to be inversely proportional to the sulfur flow rate. According to the Nukiyama-Tanasawa equation (1939), the spray droplet size $\left(d_{s}\right)$ increases with liquid flow rate $\left(Q_{l}\right)$ : i.e.,

$$
d_{s}=\frac{585}{u_{r}}\left(\frac{\sigma_{l}}{\rho_{l}}\right)^{0.5}+597\left(\frac{\mu_{l}}{\sqrt{\sigma_{l} \rho_{l}}}\right)^{0.45}\left(\frac{1000 Q_{l}}{Q_{a}}\right)^{1.5}
$$

Weiss therefore concluded that the product quality improves with sulfur droplet size.

\subsubsection{Effect of Atomizing Air on Product Quality}

Lim (1978) found that the product quality decreased as the atomizing air flow rate was increased. Weiss found this relationship to be linear when his results were normalized to take into account the effect of sulfur flow rate on product quality (see Figure 2.2). Using the relationship given by Nukiyama and Tanasawa (Equation (2.1)), Weiss confirmed that smaller spray droplet sizes gave rise to a lower product quality (or higher $D_{25}$ values).

The present hydrodynamic study (see Chapter 5) showed that the atomizing air flow rate could significantly influence the solids circulation and the size of the spray zone, in addition to the spary droplet size. Moreover, the spray angle was observed to vary with atomizing air flow rate. These effects of atomizing air flow rate could ultimately alter the product quality; however, such effects were not addressed in the previous studies. 


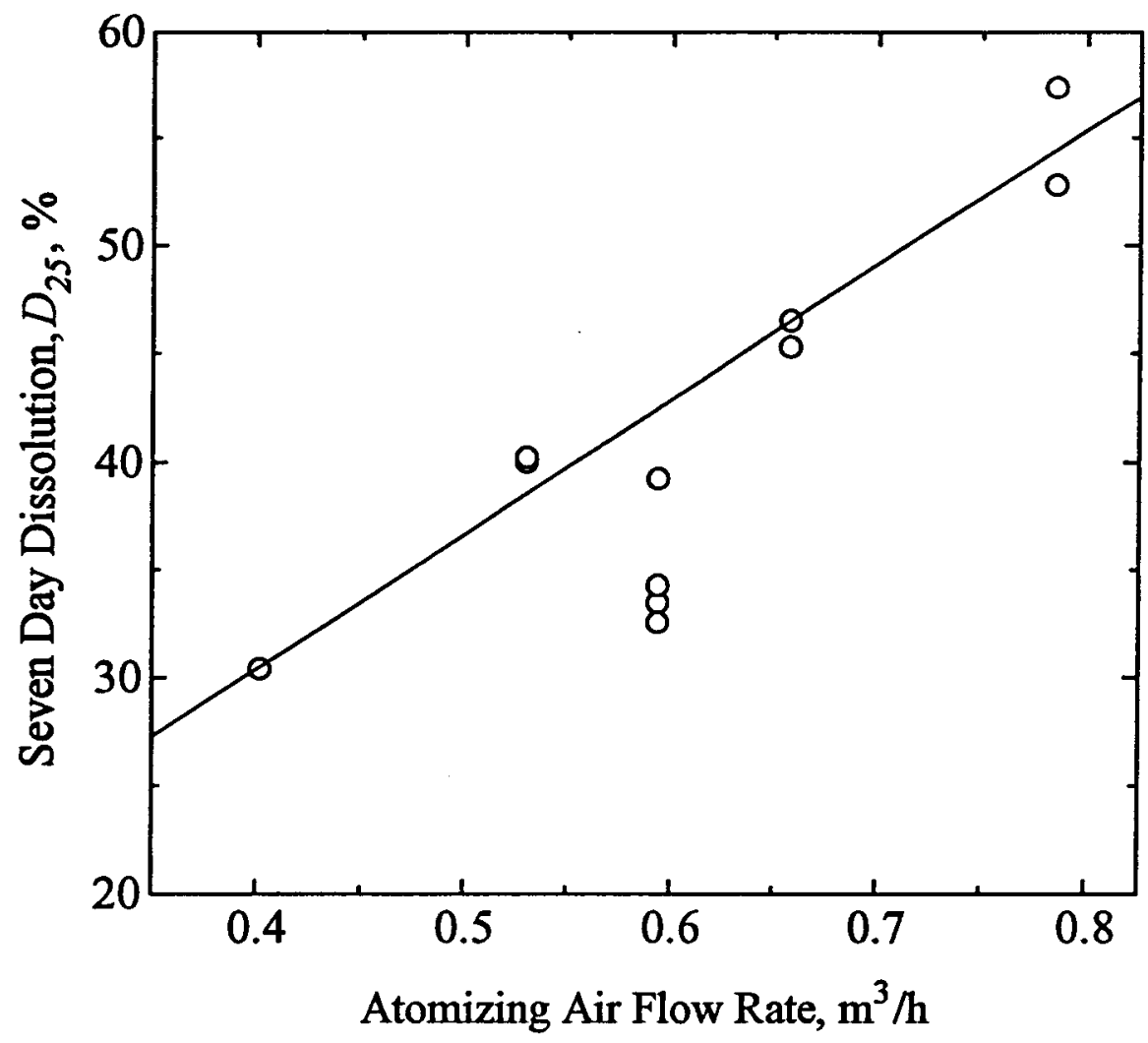

Figure 2.2: Effect of atomizing air flow rate on dissolution rate adjusted to a reference sulfur flow rate (Weiss, 1981).

\subsubsection{Effect of Bed Depth on Product Quality}

The batch studies conducted by Lim (1978) suggested that reducing the bed height improves the product quality. Shorter particle cycle times associated with shallower beds were thought to increase the chance of particles receiving uniform coats and hence improve the product quality.

However, Weiss' experimental results showed that the bed height had little effect on product quality. In his investigation on the effect of bed depth, the spouting air flow rate had to be increased with bed depth to maintain spouting (other variables were fixed). Increasing the spouting air flow rate, according to Mathur and Epstein (1974), increases the urea 
circulation rate and consequently lowers the sulfur deposition on the urea particles per pass. owever, Weiss found that the changes resulting from the variation in bed depth and spouting air flow rate did not alter the product quality significantly.

Tsai found the bed depth to have a more pronounced influence on the product quality in the continuous process. In oder to explain his results, Tsai introduced the concept of the "spray zone". The spray zone was assumed to cover the lower portion of the spout and consequently the particles that enter the spout above the spray zone did not receive any additional coating. As the bed height increases, more particles by-pass the spray zone, resulting in more inadequately coated particles and a lower product quality.

The concept of the "spray zone" is explored in the development of the mathematical models in Chapter 4.

\subsubsection{Effect of Spouting Air Flow Rate on Product Quality}

Lim (1978) found that the spouting air flow rate had little effect on the product quality in the batch coating process. Weiss and Tsai did not investigate the effect of the spouting air flow rate.

\subsubsection{Effect of Chemical Additives on Product Quality}

Silicone (Dow Corning 200) was found to improve the product quality according to Weiss (1981). Other chemical additives including $\mathrm{CO}_{2}, \mathrm{NH}_{3}, \mathrm{~N}_{2}$ and liquid dicyclopentadiene resulted in no major improvements in product quality in the study conducted by $\operatorname{Lim}(1978)$.

\subsection{Models of Spouted Bed Coating Process}

Basically three approaches for modeling spray coating in spouted beds have been reported in the past. 
The earliest paper was published by Umaki and Mathur in 1976. The model of a continuous granulation process was based on mass and number balances which took into account particle growth by solute deposition, particle breakage and dust formation due to particle abrasion and undeposited spray droplets. The model correlated the experimental particle growth rate data reasonably well. Unfortunately, as pointed out by Mann (1978), the assumptions of constant bed weight, and constant ratio of the formation rate of fresh nuclei to the number of particles in the bed implied that the particle growth resulted in a reduction of the formation of fresh nuclei, which would ultimately lead to just one or two large particles in the bed. This model was also limited to predicting the mean bed particle size. The particle size distribution, which is an important feature of slow release fertilizers, could not be predicted.

In 1983, Mann developed a model which predicted the coating distribution of the solids produced in a spout-fluid bed equipped with a draft tube and operating in batch mode. Mann believed that the coating distribution is mainly affected by the number distribution of passages through the spray zone and the distribution of the coating mass deposited on the particles per passage. Based on the findings of Cox (1967) and Mann (1974, 1975, 1979 and 1981) that the latter two distributions asymptotically approached normal distributions with time, Mann developed a 5-parameter model. The parameters were operating time, mean and variance of cycle time, and mean and variance of coating amount per cycle. Mann suggested 90 short experimental runs to relate the latter four variables to bed diameter, annulus width, height of draft tube from the base, atomizer type, air flow rate, and coating solution flow rate.

For a coating process in which the coat itself does not significantly add to the size of the bed particles and for a fixed bed geometry, this approach appears to be valid. However, if the coating material significantly increases the size of the bed particles, short experiments may not give proper indication of the effect of the latter four variables. The increase in the 
particle size results in particles receiving more coating material per cycle; this adds to the bed height which may, in turn, decrease the cycle time of the bed particles. Moreover, as the model is modified for continuous operation, additional operating variables may need to be considered. In such cases, the number of experiments required to determine the relationship between the operating variables and the model variables will increase significantly. Such an increase in the number of experiments may render this approach ineffective for practical purposes.

In 1984, Berruti et al. developed a mathematical model for predicting the size distribution of solids formed in a continuous spouted bed coating process. They assumed perfect particle mixing, constant feed composition (i.e., feed rates of coating material and bed particles), no particle segregation at the product discharge, constant hold up and negligible particle breakage and fines formation. On the basis of a particle population balance, which was developed by Randolph and Larson (1971) for describing a crystallization process, the model predicted the size distribution of the bed particles under transient conditions. Prior knowledge of the feed rate, mean feed particle size and growth rate were required to determine the product size distribution. The authors kept the growth rate and the mean feed particle size constant while altering the feed rate. Their results showed that the size distribution of the product particles approached a log-normal distribution.

Two assumptions, namely perfect particle mixing and a well-dispersed homogeneous gas phase containing the coating agent, seem, however, unrealistic for the spouted bed processes.

None of the three aforementioned models considered, in detail, the coating mechanisms and particle circulation patterns inside the spouted bed. Umaki and Mathur (1976), and Berruti et al. (1984) treated the bed as a perfectly stirred vessel and assumed that particles in all regions of the bed received the same amount of coating. Mann (1983) assumed a 
spray zone, but his model variables were empirical. These variables could not explain the particle circulation in the bed and the coating mechanism.

\subsection{Models and Correlations for Spouted Bed Hydrodynamics}

Since only a few hydrodynamic models and correlations for shallow spouted beds are available in the literature, those that are widely used for standard spouted beds are also considered here. It should be noted that detailed and critical review of most hydrodynamic models and correlations considered here can be found elsewhere (Mathur and Epstein, 1974; Epstein and Grace, 1984; Krzywanski, 1992).

\subsubsection{Minimum Spouting Velocity}

For cylindrical vessels up to about $0.6 \mathrm{~m}$ in diameter with conical base, the most reliable (within $\pm 10 \%$ ) correlation for the minimum spouting velocity is, according to Epstein and Grace (1984), the Mathur-Gishler (1955) equation:

$$
U_{m s}=\frac{d_{p}}{D}\left(\frac{d_{i}}{D}\right)^{1 / 3} \sqrt{2 g H \frac{\rho_{p}-\rho}{\rho}}
$$

Wu et al. (1987) modified the Mathur-Gishler equation by separating the density and bed height terms in the following way:

$$
U_{m s}=k \sqrt{2 g H}\left(\frac{d_{p}}{D}\right)^{\alpha}\left(\frac{d_{i}}{D}\right)^{\beta}\left(\frac{H}{D}\right)^{\gamma}\left(\frac{\rho_{p}-\rho}{\rho}\right)^{\delta}
$$

The constants $k, \alpha, \beta, \gamma$, and $\delta$ were calculated using a standard least squares technique to fit 112 data points. The values of the constants are $10.6,1.05,0.266,-0.095$ and 0.256 , respectively. The modified equation improved the $U_{m s}$ predictions significantly, especially at temperatures well above ambient. 


\subsubsection{Solids Circulation and Bed Hydrodynamics}

Basically three approaches for predicting the particle circulation rates have been reported in the literature. Two approaches, according to Morgan et al. (1985), involve using onedimensional particle force, and mass and momentum balances in the spout. A more recent and rigorous approach is based on the theory of plasticity for the solids motion in the annulus (Krzywanski et al., 1992; Amirshahidi, 1984; Khoe, 1980). The former two approaches also predict fluid velocity and voidage profiles in the spout, while the latter approach does not. However, Krzywanski et al. (1992) combined the theory of plasticity with the vector Ergun (1952) equation in the annulus and the two-phase momentum equations in the spout to solve for the bed hydrodynamics.

The most recent force balance model developed by Lim and Mathur (1978) had problems with the stiffness of the model equations at the bed inlet, although its predictions using experimental values away from the inlet as the initial conditions were reasonable. Khoe (1980) and Amirshahidi (1984) determined the solids flow in the annulus based on the theory of plasticity. The model developed by Khoe was strongly dependent on experimental results - the magnitudes and locations of sources and sinks were found experimentally. Amirshahidi encountered difficulties in the computation of the stress field in the conical region which is required to calculate the velocity field of solids. The theory of plasticity was applied to solve solids flow in the annulus; the solids flow in the rest of the bed would require additional equations. The Krzywanski et al. model required basic information such as wall and internal friction angles as well as the fluid velocity profile at the fluid inlet, which are not easily calculated nor readily available.

Although the model developed by Krzywanski et al. is the most comprehensive model available, applying it to the current study requires extraordinary computational resources. Furthermore, the model must be corrected for the unusual geometry at the bed inlet due to 
the presence of the nozzle in the coating unit (see Chapter 5). Accounting for the nozzle is a complex task because the flow rates of atomizing air and spouting air, the location and type of the spray nozzle all affect the boundary conditions of the model. Moreover, the bed hydrodynamics were found to be very sensitive to the friction angles which probably vary with the amount of sulfur on the particles.

The mass and momentum balances model, on the other hand, is much simpler, and yet, it was generally found to provide good approximations of solids circulation and bed hydrodynamics with little or no modification (Lefroy and Davidson, 1969; Morgan et al., 1985; Stocker, 1987). This model, however, required accurate estimates of spout diameter $\left(D_{s}\right)$, pressure distribution in the spout $\left(P_{s}\right)$, particle-fluid interaction $\left(\beta_{p}\right)$ and air flow into the annulus $\left(U_{r}\right)$. The correlations for the first two of these variables are reviewed in the next two sections.

\subsubsection{Spout Diameter}

Although several spout shapes have been observed (Mathur and Epstein, 1974), a constant spout diameter has been commonly assumed (Epstein and Grace, 1984). The most recent correlation of the average spout diameter was developed by Wu et al. (1987) which is a modification of the equation given by Bridgewater and Mathur (1972). The modified equation resulted from a new set of fitted constants determined by applying a least squares fit to their data (which were obtained with $D=0.154 \mathrm{~m} ; \rho_{p}=2600 \mathrm{~kg} / \mathrm{m}^{3} ; 0.945<d_{p}<$ $1.665 \mathrm{~mm} ; 12.7<d_{i}<26.6 \mathrm{~mm} ; 0.168<H<1.38 \mathrm{~m} ; 0.168<\rho<1.259 \mathrm{~kg} / \mathrm{m}^{3} ; 10.9<\mu$ $\left.<32.0 \times 10^{-6} \mathrm{~kg} / \mathrm{m} \cdot \mathrm{s}\right)$, i.e.

$$
D_{s}=5.61 G^{0.433} D^{0.583} \mu^{0.133} /\left(\rho_{b} \rho g\right)^{0.283}
$$

where $G=\rho U$

and $\quad \rho_{b}=\rho_{p}\left(1-\varepsilon_{m f}\right)$. 
This equation was found to give better predictions of their data at elevated temperatures than the McNab (1972) equation.

\subsubsection{Pressure Profile in Annulus}

Several pressure distribution correlations have been reported in the literature. Lefroy and Davidson (1969) used an empirical correlation based on the pressure measurements at the spout-annulus interface. The only model with a strong theoretical basis was developed by Epstein and Levine (1978); it was derived from the Ergun equation (1952) and the Mamuro and Hattori flow correlation (1968), and is given by

$$
\begin{aligned}
\frac{P_{a}-P_{H}}{-\Delta P_{f}}= & \frac{1}{h\left(2 \alpha_{p}-1\right)}\left[2 ( \alpha _ { p } - 1 ) \left[1.5\left(h^{2}-x^{2}\right)-\left(h^{3}-x^{3}\right)\right.\right. \\
& \left.+0.25\left(h^{4}-x^{4}\right)\right]+3\left[3\left(h^{3}-x^{3}\right)-4.5\left(h^{4}-x^{4}\right)\right. \\
& \left.\left.+3\left(h^{5}-x^{5}\right)-\left(h^{6}-x^{6}\right)+0.143\left(h^{7}-x^{7}\right)\right]\right]
\end{aligned}
$$

where $\alpha_{p}=2+\frac{129 \mu\left(1-\varepsilon_{m f}\right)}{\rho d_{p} U_{m f}}$,

$$
h=H / H_{m},
$$

and $x=z / H_{m}$.

This equation describes the pressure distribution in the annulus but, since there is a relatively small pressure drop between the spout and annulus (Rovero et al., 1985), it can also be used to estimate the pressure distribution in the spout.

Morgan and Littman (1980) developed the following general pressure drop correlation based on experimental pressure measurements reported in the literature:

$$
\Delta P_{m s} / \Delta P_{m f}=1-Y
$$

where

$$
Y^{2}+[2(X-0.2)-1.8+(3.24 / \varphi)] Y+[(X-2)(X-0.2)-(3.24 X / \varphi)]=0,(2 .
$$




$$
X=1 /[(H / D)+1]
$$

and $\quad \varphi=7.18\left(\frac{\rho U_{m f} U_{t}}{\left(\rho_{p}-\rho\right) g d_{i}} \phi_{s}\left(5 \phi_{s}^{3}-7.57 \phi_{s}^{2}+4.09 \phi_{s}-0.516\right)-\frac{d_{i}}{D}\right)+1.07$

Fluid flow models for the annulus can also be modified to predict the pressure distribution with the aid of expressions such as the Ergun (1952) equation. Mamuro and Hattori (1968) derived a fluid flow model for the annulus using Darcy's law and Rovero et al. (1983) modified the Mamuro-Hattori equation for beds having a conical base by substituting

$$
A_{a}=\pi\left(D^{2}-D_{s}^{2}\right) / 4=A_{0}, \text { for } z>H_{c}
$$

and

$$
A_{a}=\pi\left[\left(2 z \tan (\theta / 2)+D_{b}\right)^{2}-D_{s}^{2}\right] / 4, \text { for } z \leq H_{c}
$$

into $\frac{d Q_{a}}{d z}\left(\frac{d^{2} Q_{a}}{d z^{2}}\right)=\frac{B Q_{a}}{A_{a}}-B U_{a H_{m}}$

where $B=18 U_{a H_{m}} A_{a}^{2} / H_{m}^{3}$

and $\quad Q_{a}=A_{a} U_{a}$

The boundary conditions are:

$$
\begin{aligned}
& Q_{a}=Q_{a 0} \text { at } z=0 \\
& Q_{a}=U_{a H_{m}} A_{a} \text { at } z=H_{m}
\end{aligned}
$$

\subsection{Coating Mechanism}

Four mechanical collection processes ${ }^{4}$ have been reported in the literature (Clift et al., 1981 ; Lunde and Lapple, 1957): diffusional deposition, inertial deposition, direct interception and gravitational settling. These processes are often aided by electrophoretic, thermophoretic and diffusiophoretic effects (Meisen et al., 1971). However, only the inertial deposition mechanism is considered because it was inferred that it was the dominating

\footnotetext{
4 collection process, in this study, corresponds to the mechanisms by which the atomized sulfur droplets deposit onto the bed particles.
} 
mechanism under the experimental conditions prevailing in this study. Thus, the review in this section covers more recent inertial deposition correlations reported in the literature.

According to Clift et al. (1981), the correlation given by Thambimuthu (1980) is the most reliable equation for predicting the inertial impaction efficiency of a single spherical collector:

$$
\eta=\left(S_{t} /\left(S_{t}+0.062 \varepsilon\right)\right)^{3}, \text { for } 0.002<S_{t}<0.02
$$

where $S_{t}=(u-v) d_{c}^{2} \rho_{c} / 18 \mu d_{p}$

The range of Stokes numbers $\left(S_{t}\right)$, however, is rather small and is not generally applicable to the conditions of this study.

Earlier work by Behie et al. (1972) led to equations which are valid for a wider range of Stokes numbers:

$$
\begin{aligned}
& \eta=0, \text { for } S_{t} \leq 0.083 \\
& \eta=0.0036-0.2323 S_{t}+2.422 S_{t}^{2}-2.033 S_{t}^{3}, \text { for } 0.083<S_{t} \leq 0.6 \\
& \eta=S_{t}^{2} /\left(S_{t}+0.5\right)^{2}, \text { for } S_{t}>0.6
\end{aligned}
$$

These equations for the single particle collection efficiency were obtained based on the assumption that the aerosols were rigid and spherical.

\subsection{Monte Carlo Method}

The term "Monte Carlo" was introduced by von Neumann and Ulam during World War II, as a code word for the secret work at Los Alamos; it was suggested by the gambling casinos at the city of Monte Carlo in Monaco (Rubinstein, 1981). The Monte Carlo method has been often confused with "stochastic simulation", and Ripley (1987) suggested that the term "Monte Carlo method" should have the more specialized meaning of "doing something clever and stochastic with simulation". Rubinstein (1981) defines stochastic simulation as statistical sampling experiments with a model over time which involves the use of a 
random number; Monte Carlo simulation as a technique using random or pseudorandom numbers for solving model equations. The latter definition is used to describe the model developed in this work.

Although this type of simulation is often viewed as a "method of last resort" to be employed when everything else has failed, recent advances in simulation methodologies, availability of software, and technical developments have made Monte Carlo simulation one of the most widely used and accepted tools in system analysis and operations research (Rubinstein, 1981). The reasons for using the Monte Carlo method, in the past, included

(i) The data are impossible or very expensive to obtain (e.g. the performance of largescale rocket engines and the effect of proposed tax cuts on the economy);

(ii) The system cannot be described in terms of a set of mathematical equations for which analytic solutions are obtainable;

(iii) The solution to a mathematical model cannot be obtained by straight-forward analytic techniques;

(iv) The experimental verification of the mathematical models describing the system is impossible or very costly to perform.

In this work, the Monte Carlo method was used because of reason (iii). 


\section{Chapter 3. \\ Experimental Materials, Apparatus and Procedures}

The coating apparatus developed by Meisen et al. (1986) was used for all experiments conducted in this study. Minor modifications to the spouted beds were necessary for the study of bed hydrodynamics and a spray box was built to determine size distribution of the spray droplets. The experimental procedures used for the coating studies were similar to those described by previous workers (Weiss, 1981 and Tsai, 1986). Since the apparatus used in this work was largely the same as that used by previous workers, more emphasis is placed, in this chapter, on describing the modifications to the equipment.

\subsection{Experimental Materials}

Urea and sulfur are the only materials used to produce sulfur coated urea in this study; wax, silicone and other chemical additives were not needed. Urea and sulfur coated urea particles are shown in Plates 3.1 - 3.3. The chemical and physical properties of sulfur and urea are important in determining various operating limits of the coating process, and are discussed in the following subsections.

Initially, only urea and sulfur coated urea (SCU) were considered for the hydrodynamics study; however, considerable attrition was encountered with these particles. Therefore, polyformaldehyde, polyethylene and polystyrene particles in addition to the urea and SCU particles, were also used for this purpose. They are shown in Plates 3.4, 3.5 and 3.6, respectively, and discussed in Section 3.1.3. 


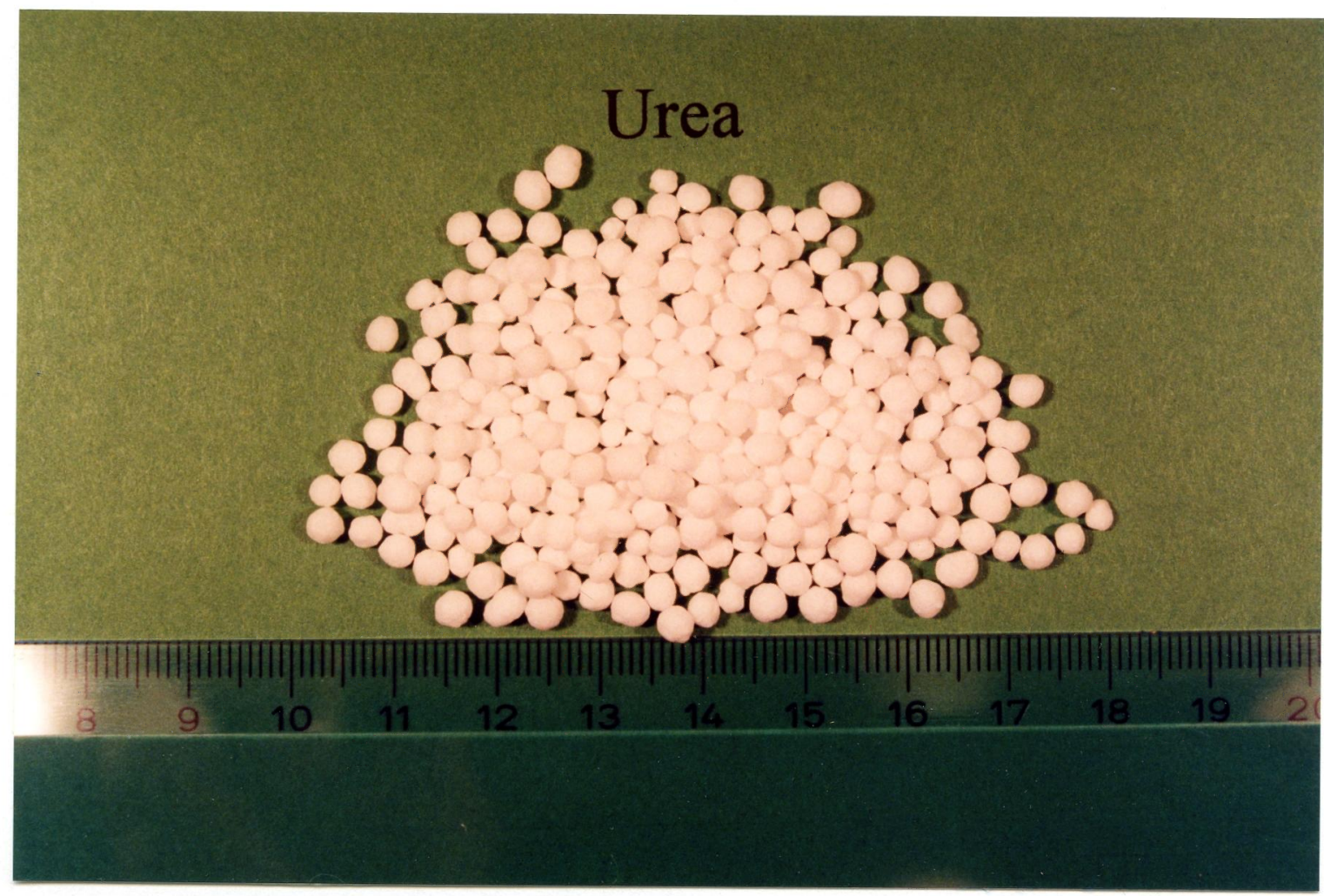

Plate 3.1: Urea.

\section{Sulfur Coated Urea} (Batch Product)

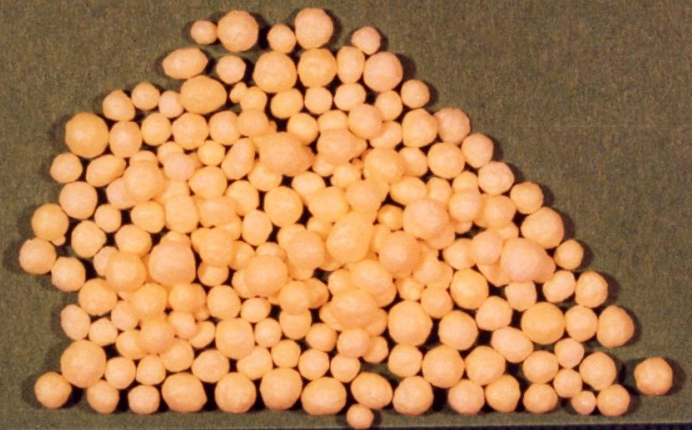

Plate 3.2: Sulfur coated urea produced by batch process. 


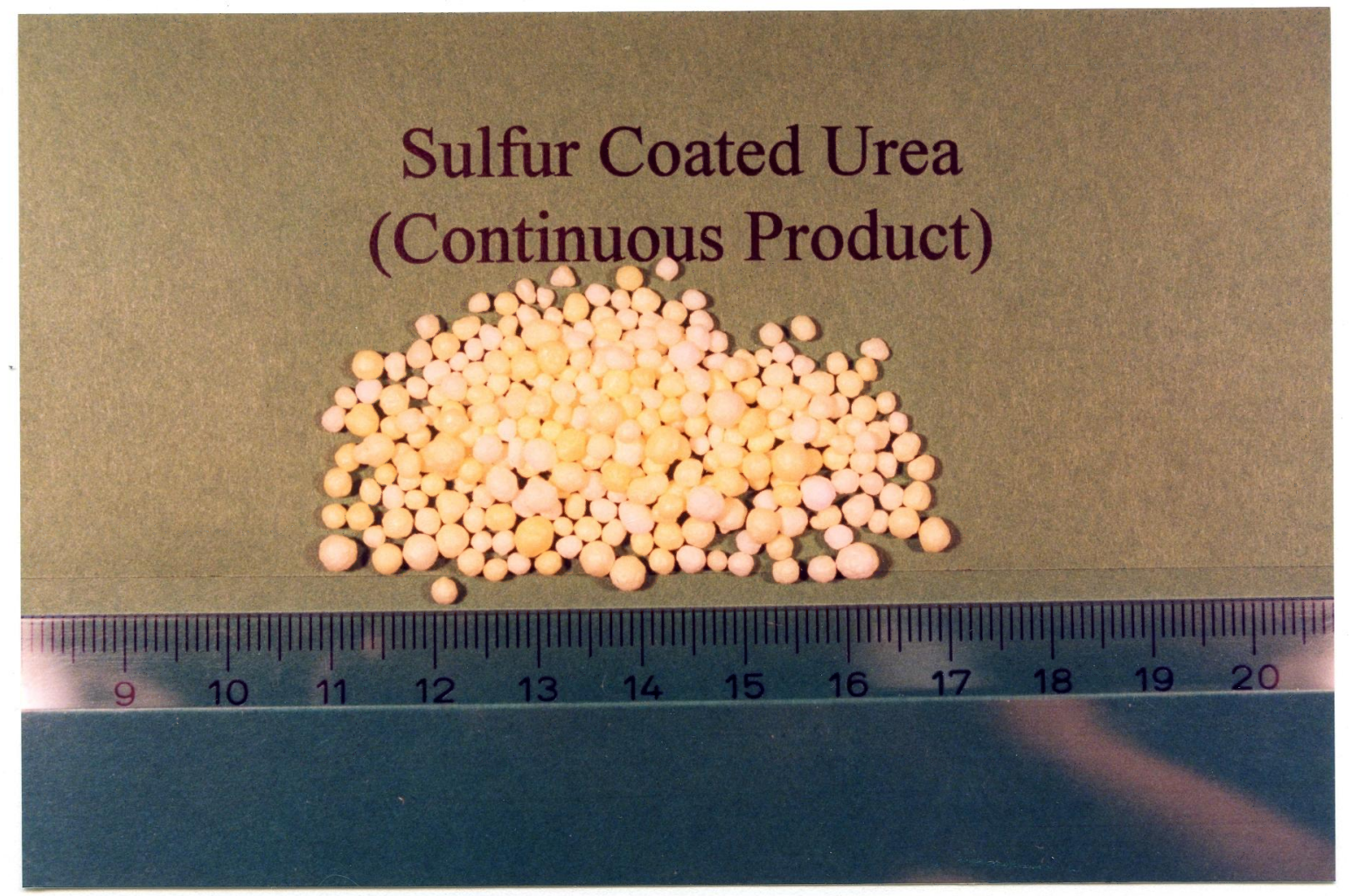

Plate 3.3: Sulfur coated urea produced by continuous process.

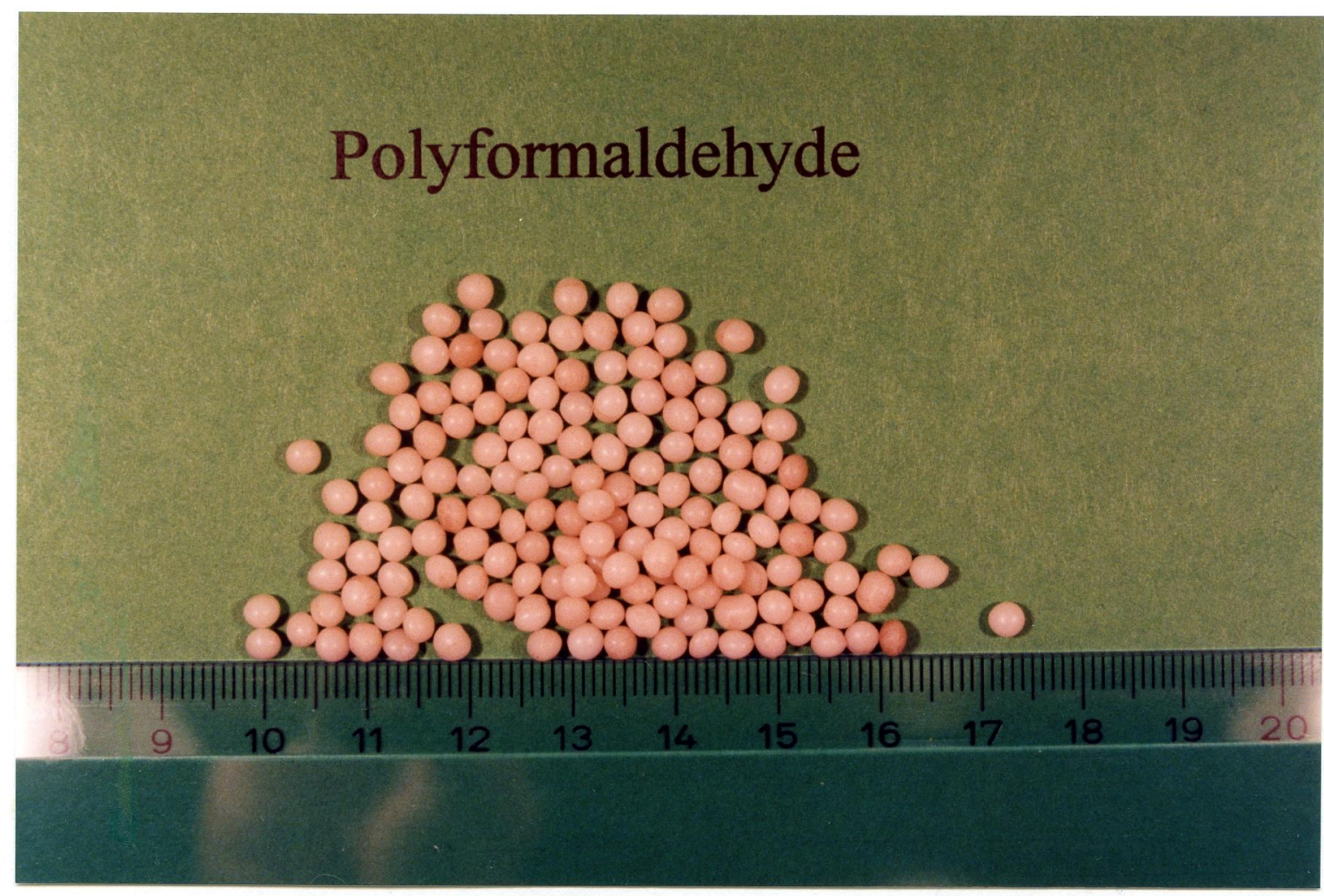

Plate 3.4: Polyformaldehyde. 


\section{Polyethylene}
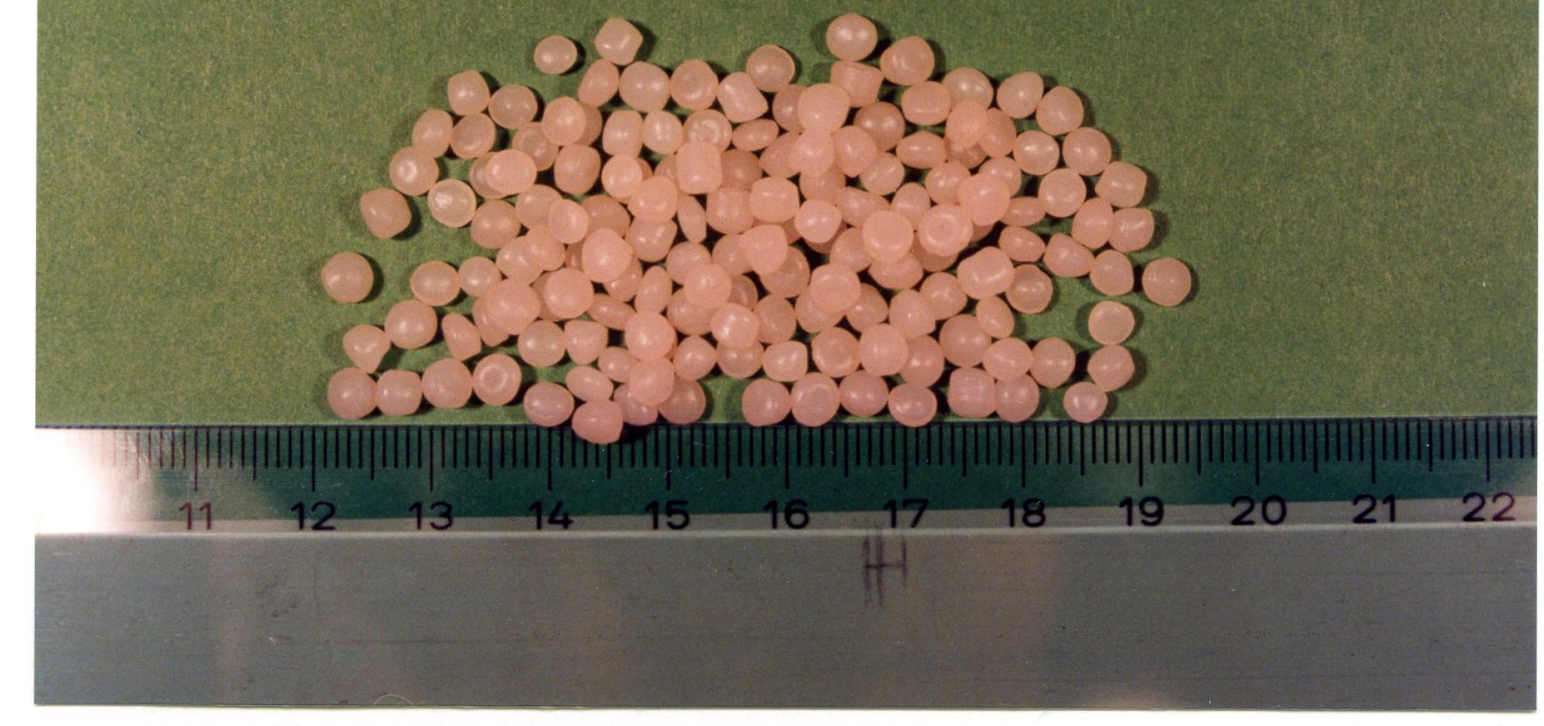

Plate 3.5: Polyethylene.

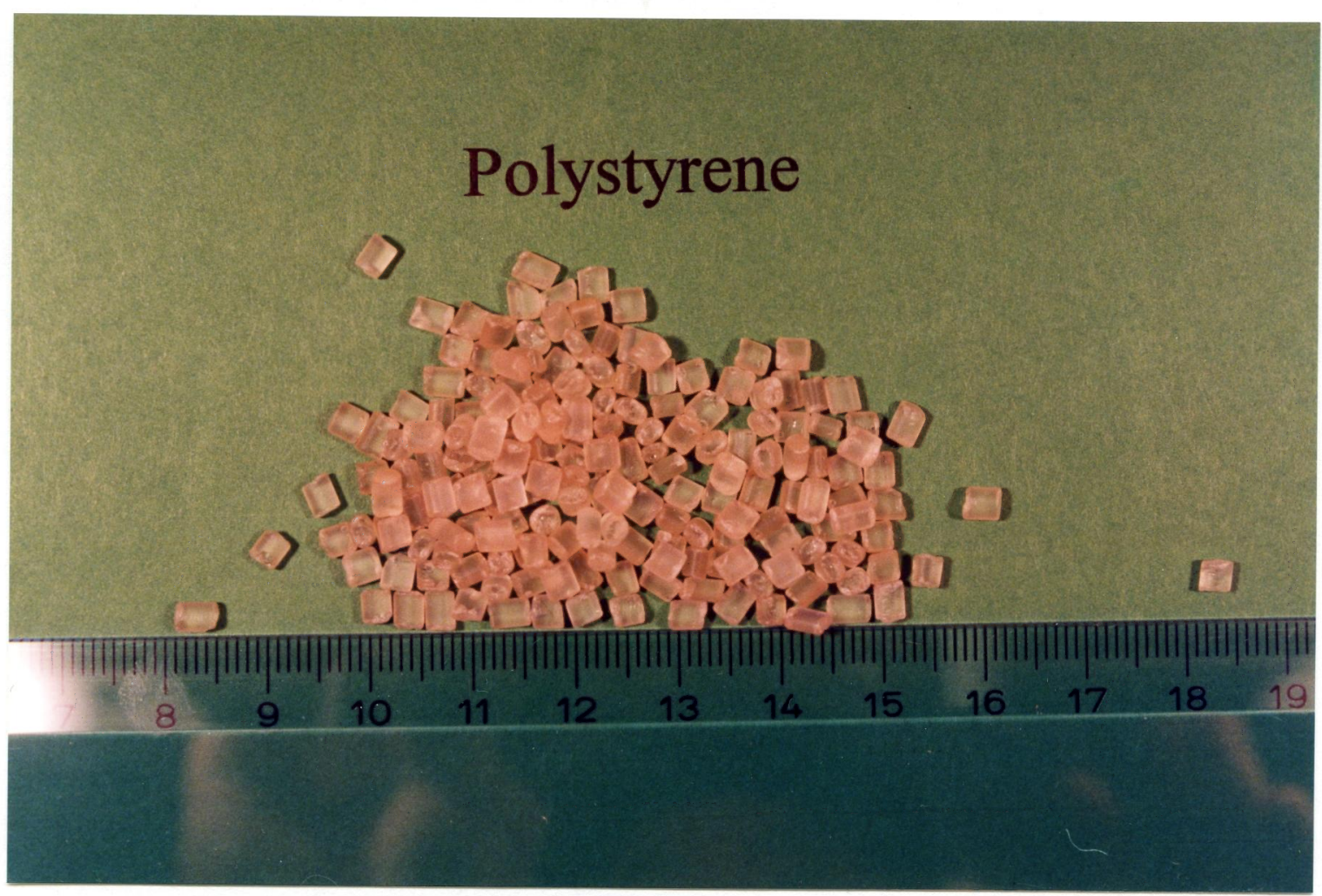

Plate 3.6: Polystyrene. 


\subsubsection{Urea}

Urea was supplied by Sherritt-Gordon Ltd. and produced by the NSM fluidized bed granulation process. Selected properties of urea are listed in Table 3.1.

The operating bed temperature was kept above $60^{\circ} \mathrm{C}$. Heavy attrition was observed at room temperatures, i.e., dust build up on the Plexiglas column was observed almost immediately after spouting started. The attrition rate gradually decreased with increasing temperature; however, no data were collected to determine the effect of temperature on the attrition rate. The lower operating limit of $60^{\circ} \mathrm{C}$ was adopted based on visual inspection of dust build up on the column walls.

Other factors that appeared to influence the attrition rate were spouting air flow rate, spouting air orifice diameter, and bed diameter. Higher spouting air flow rates, small orifices, and smaller bed diameter increased the attrition rate.

Table 3.1: Selected physical properties of urea.

Melting point (Perry et al., 1984)

$133^{\circ} \mathrm{C}$

Sphericity

Particle density (Perry et al., 1984)

Size distribution (Sherritt-Gordon Ltd.)

\begin{tabular}{rcc} 
Mesh (CDN) & Aperture Size $(\mathrm{mm})$ & Wt. fraction $(\%)$ \\
\hline+6 & 3.36 & 2.8 \\
$-6+7$ & 2.83 & 24.5 \\
$-7+8$ & 2.38 & 33.5 \\
$-8+10$ & 2.0 & 30.1 \\
$-10+16$ & 1.19 & 9.1 \\
-16 & & trace
\end{tabular}

mean size $=\left[\sum_{i} x_{i} / d_{p i}\right]^{-1} \quad 2.16$ 


\subsubsection{Sulfur}

PETROSUL International Ltd. provided sulfur for this work. The sulfur purity exceeded $99.5 \mathrm{wt} \%$ due to the presence of only minor traces of ash and carbon (total of $0.10 \%$ on average).

Selected properties of solid and liquid sulfur are given in Tables 3.2 and 3.3, and Figure

Table 3.2: Selected chemical and physical properties of sulfur (Stauffer Chemical Co.).

\begin{tabular}{|l|l|}
\hline Physical state (@ 21 $\left.{ }^{\circ} \mathrm{C}, 1 \mathrm{~atm}\right)$ & Solid \\
\hline Bulk density, $\mathrm{kg} / \mathrm{m}^{3}$ & $1200-1394$ Lumps, 560 - 960 Powder \\
\hline Boiling point & $444^{\circ} \mathrm{C}$ \\
\hline Melting point & $119^{\circ} \mathrm{C}$ (approximate) \\
\hline Odor & None \\
\hline Flash point & $188^{\circ} \mathrm{C}, \mathrm{COC}$ \\
\hline Auto ignition temperature (dust in air) & $190^{\circ} \mathrm{C}$ \\
\hline Vapor pressure @ 20 $0^{\circ} \mathrm{C}$ & $<0.0001 \mathrm{~mm} \mathrm{Hg}$ \\
\hline Explosive limits (dust in air) & between 35 and $1400 \mathrm{~g} / \mathrm{m}^{3}$ \\
\hline
\end{tabular}

Table 3.3: Properties of common sulfur allotropes (Donahue and Meyer, 1965; Dale and Ludwig, 1965).

\begin{tabular}{|l|c|c|}
\hline Property & $\mathrm{S}_{\alpha}$ & $\mathrm{S}_{\beta}$ \\
\hline Common name & Orthorhombic sulfur & Monoclinic sulfur \\
\hline Recommended name & Orthorhombic $(\alpha)$ sulfur & Monoclinic $(\beta)$ sulfur \\
\hline Molecular formula & $\mathrm{S}_{128}$ & $\mathrm{~S}_{48}$ \\
\hline Crystalline form & Orthorhombic & Monoclinic \\
\hline Unit cell & 16 molecules of $\mathrm{S}_{\lambda}\left(\mathrm{S}_{8}\right)$ & 6 molecules of $\mathrm{S}_{\lambda}\left(\mathrm{S}_{8}\right)$ \\
\hline Stability region & $<95.5^{\circ} \mathrm{C}$ & $95.5^{\circ} \mathrm{C}$ to $119^{\circ} \mathrm{C}$ \\
\hline Color & Opaque yellow at $24^{\circ} \mathrm{C}$ & Between yellow and orange \\
\hline Density, kg/m 3 & 2070 & 1960 \\
\hline Shore B-2 hardness & 90 & 1.96 \\
\hline Tensile strength, $\mathrm{kPa}$ & 330 & 410 \\
\hline \hline
\end{tabular}


3.1. The minimum sulfur viscosity occurs at $159^{\circ} \mathrm{C}$; higher temperatures result in the formation of polymeric sulfur which is very viscous as shown in Figure 3.1. The explosive limits and auto ignition temperatures were considered in designing the sulfur spray box. The sulfur allotropes listed in Table 3.3 were important in defining particle properties in the simulation of the coating process, and assessing the quality of sulfur coated urea.

\subsubsection{Particles Used in Hydrodynamics Study}

Considerable attrition was encountered with urea and sulfur coated urea (SCU) particles and, as a result, they were restricted to determining the minimum spouting velocity in the

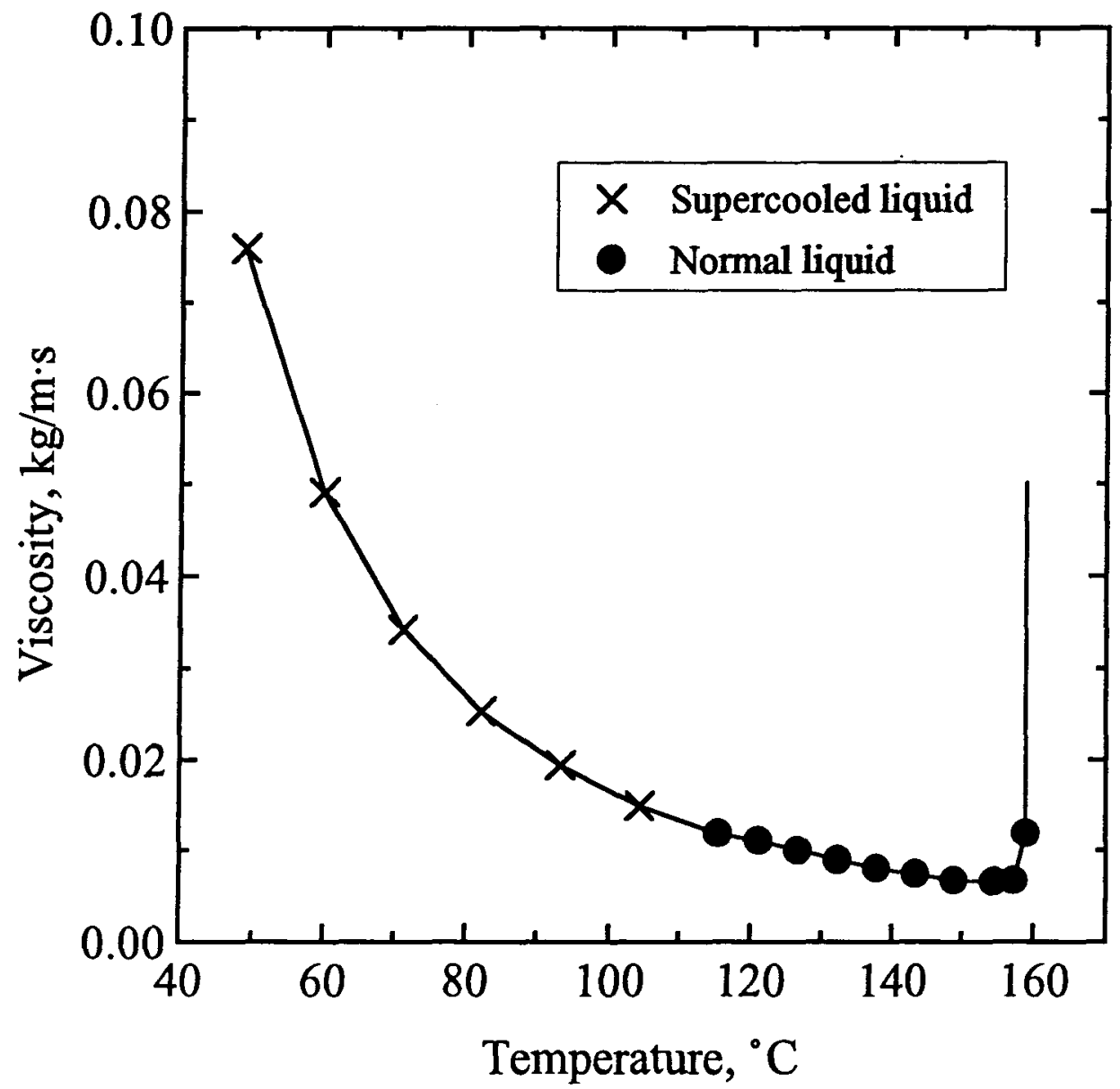

Figure 3.1: Viscosity of sulfur at low temperature range (Freeport Sulfur Co., 1954). 
Table 3.4: Physical properties of bed particles.

\begin{tabular}{|c|c|c|c|c|}
\hline Material & $\begin{array}{c}d_{n} \\
(\mathrm{~mm})\end{array}$ & $\begin{array}{c}\rho_{p}{ }^{*} \\
\left(\mathrm{~kg} / \mathrm{m}^{3}\right)\end{array}$ & $\varepsilon_{m f}$ & $\phi_{s}$ \\
\hline Urea & 2.16 & 1335 & 0.42 & 1 \\
\hline SCU, 19\% Sulfur & 2.27 & 1427 & 0.42 & 1 \\
\hline SCU, 28\% Sulfur & 2.33 & 1471 & 0.42 & 1 \\
\hline SCU, 31 \% Sulfur & 2.36 & 1490 & 0.42 & 1 \\
\hline Polyethylene & 2.80 & 927 & 0.40 & 1 \\
\hline Polystyrene & 2.30 & 1045 & 0.44 & 0.85 \\
\hline Polyformaldehyde & 2.70 & 1385 & 0.43 & 1 \\
\hline
\end{tabular}

* The density was measured in the standard way by using a pycnometer.

$0.24 \mathrm{~m}$ bed. Other materials were therefore used as well including polystyrene, polyethylene, and polyformaldehyde particles (see Plates 3.4 - 3.6) with similar size and density as urea. Attrition was also encountered with polystyrene, and its study was limited to determining the hydrodynamics in the $0.24 \mathrm{~m}$ bed. The physical properties of these particles are given in Table 3.4 .

\subsection{Main Coating Apparatus}

As shown by Figure 3.2, the main components of the experimental apparatus were the spouted bed, sulfur supply system, nozzle assembly, urea feeding and product withdrawal devices, and the steam, air and water supply systems. The coating facility was rebuilt by Meisen et al. (unpublished report, 1986) incorporating some of the equipment used earlier by Tsai. The major changes included larger beds ( 0.24 and $0.45 \mathrm{~m}$ diameters), different nozzle assembly, larger sulfur melting pot, and urea feeding and product withdrawal devices. 


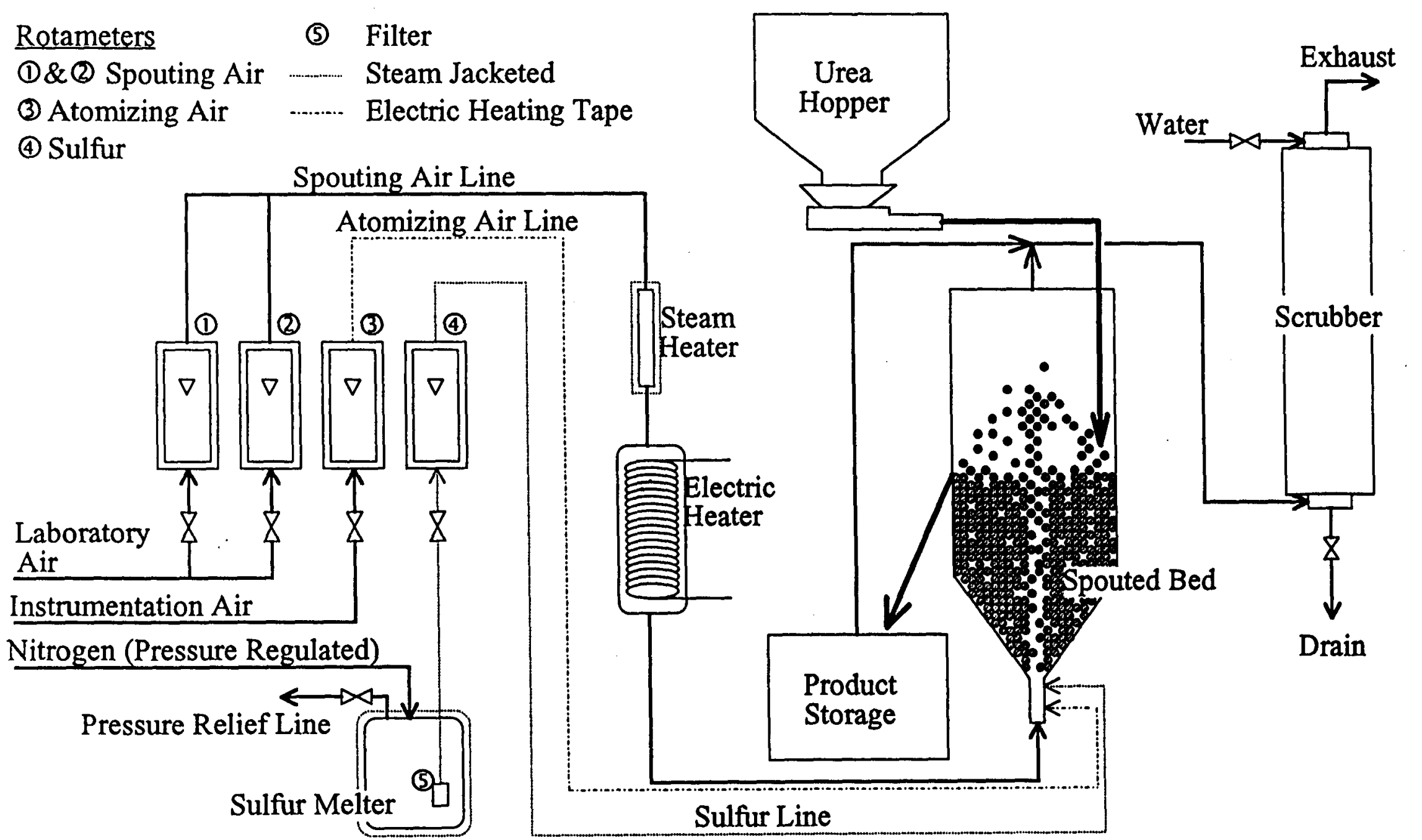

Figure 3.2: Simplified flowsheet of UBC spouted bed facility. 


\subsubsection{Spouted Bed}

The coating operation took place in a spouted bed which consisted of a Plexiglas column, a stainless steel cone with a shutter assembly, and a stainless steel cap. Columns 0.24 and $0.45 \mathrm{~m}$ in diameter were used in this study. The dimensions of the $0.24 \mathrm{~m}$ column and the cone extension for the $0.45 \mathrm{~m}$ column are given in Figure 3.3.

The $0.24 \mathrm{~m}$ column had four $25.4 \mathrm{~mm}$ dia. product discharge holes drilled at heights 0.28 , $0.36,0.44$ and $0.56 \mathrm{~m}$ from the base of the cone, while the larger column had only one discharge hole ( $32 \mathrm{~mm} \mathrm{dia}$ ) ) at the top of the cone ( $0.36 \mathrm{~m}$ from the base of the cone). Urea

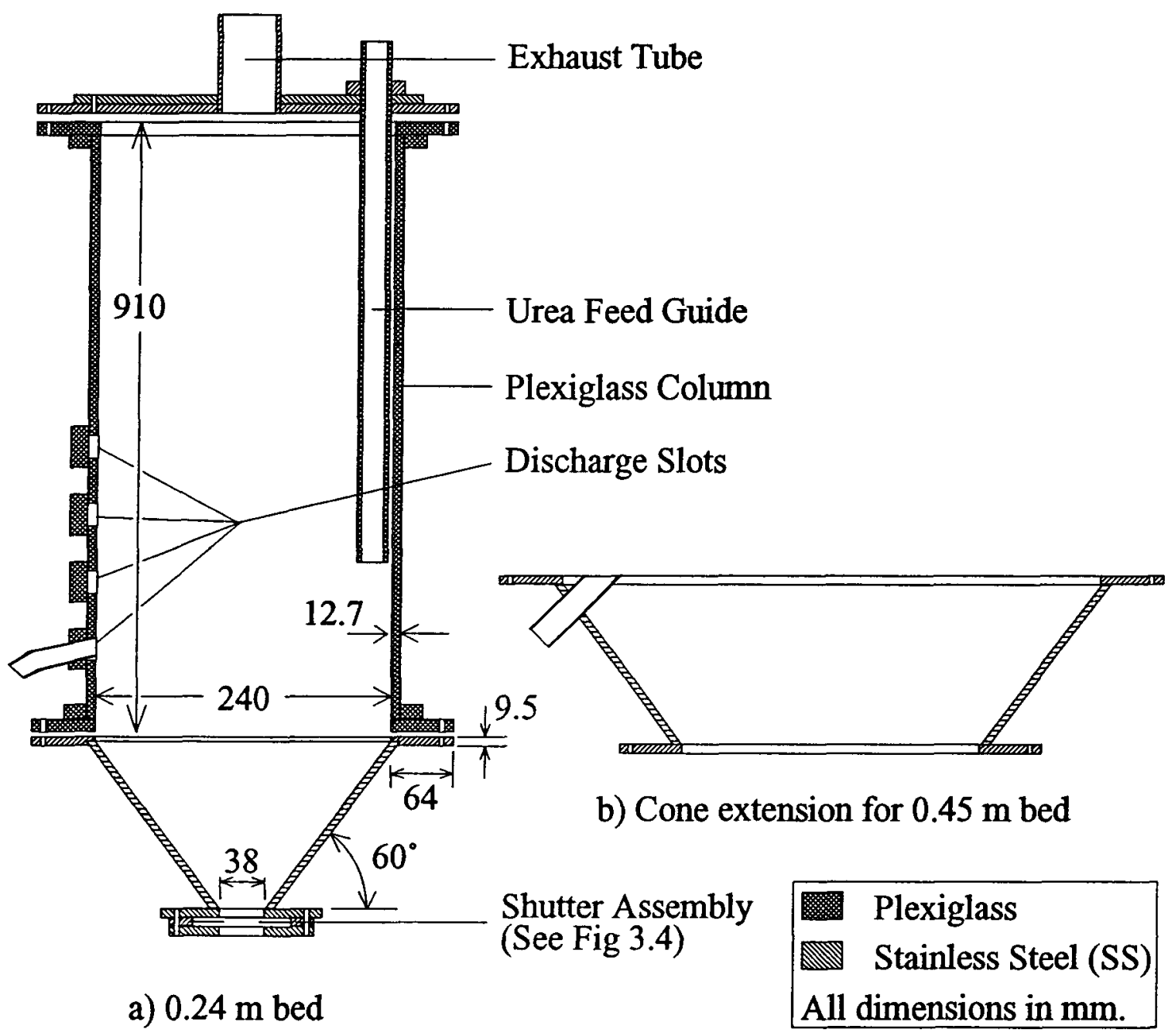

Figure 3.3: Sectional view of spouted bed column 
was fed through a Plexiglas tube opposite the product discharge locations and just above the bed. The Plexiglas column could operate continuously at temperatures up to $110^{\circ} \mathrm{C}$.

The shutter mechanism controlled the size of the orifice opening at the base of the bed. As shown in Figure 3.4, the shutter consisted of five S-shaped, overlapping stainless steel leaves arranged in a circle. The range of opening was 3.2 to $38 \mathrm{~mm}$ dia. Since the shutter
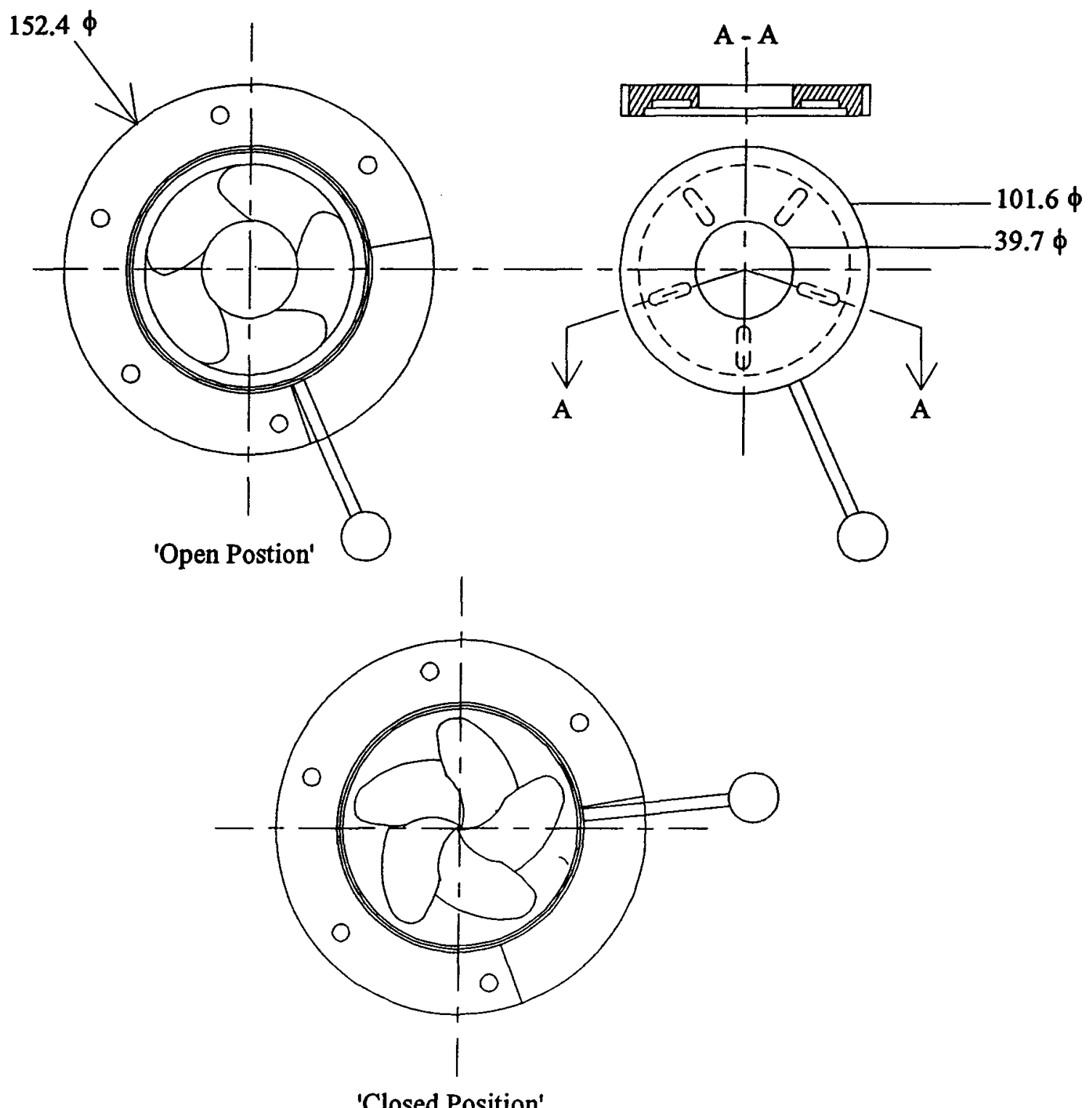

Figure 3.4: Shutter assembly (dimensions are given in $\mathrm{mm}$ ) designed by Mathur, Meisen and $\operatorname{Lim}(1978)$. 
shape changed from a circular to an irregular shape as the shutter was closed, calculating its open area required a special procedure which is given in Appendix I.

\subsubsection{Sulfur Supply System}

Only minor modifications were made to the system designed by Meisen et al. (1986). The sulfur and atomizing air lines to the upper plate had to be reinforced to allow a "slip-on" type of sulfur line connector (see Section 3.2.2.4). This type of connector was necessary for a completely steam traced sulfur line. Major components of the sulfur supply system were the sulfur melter, filter, rotameter, flow control valve, sulfur line and nitrogen supply.

\subsubsection{Sulfur Melter}

The sulfur melter used in this study was originally designed as the sulfur reservoir connected to a steam jacketed sulfur melter. Unfortunately, the steam supply was insufficient to maintain the reservoir and the melter at the desired temperature. In this work, only the sulfur reservoir was used because of its large capacity $(18.9 \mathrm{~L})$. A schematic diagram of the reservoir is shown in Figure 3.5.

The reservoir was a modified Bink 83-5404 pressure tank (0.3 m dia. OD and $0.48 \mathrm{~m}$ high), insulated with fiberglass. Holes were drilled through the cap to facilitate sulfur feeding, molten sulfur withdrawl, and pressurizing. The solid sulfur fed into the reservoir was melted by contact with a stainles steel steam coil $(9.5 \mathrm{~mm}$ dia. tube wound to $0.178 \mathrm{~m}$ dia. nine times). A full sulfur charge of $30 \mathrm{~kg}$ melted in approximately six hours. A pressure relief valve was also added on top of the reservoir to prevent excessive pressure build-up. 


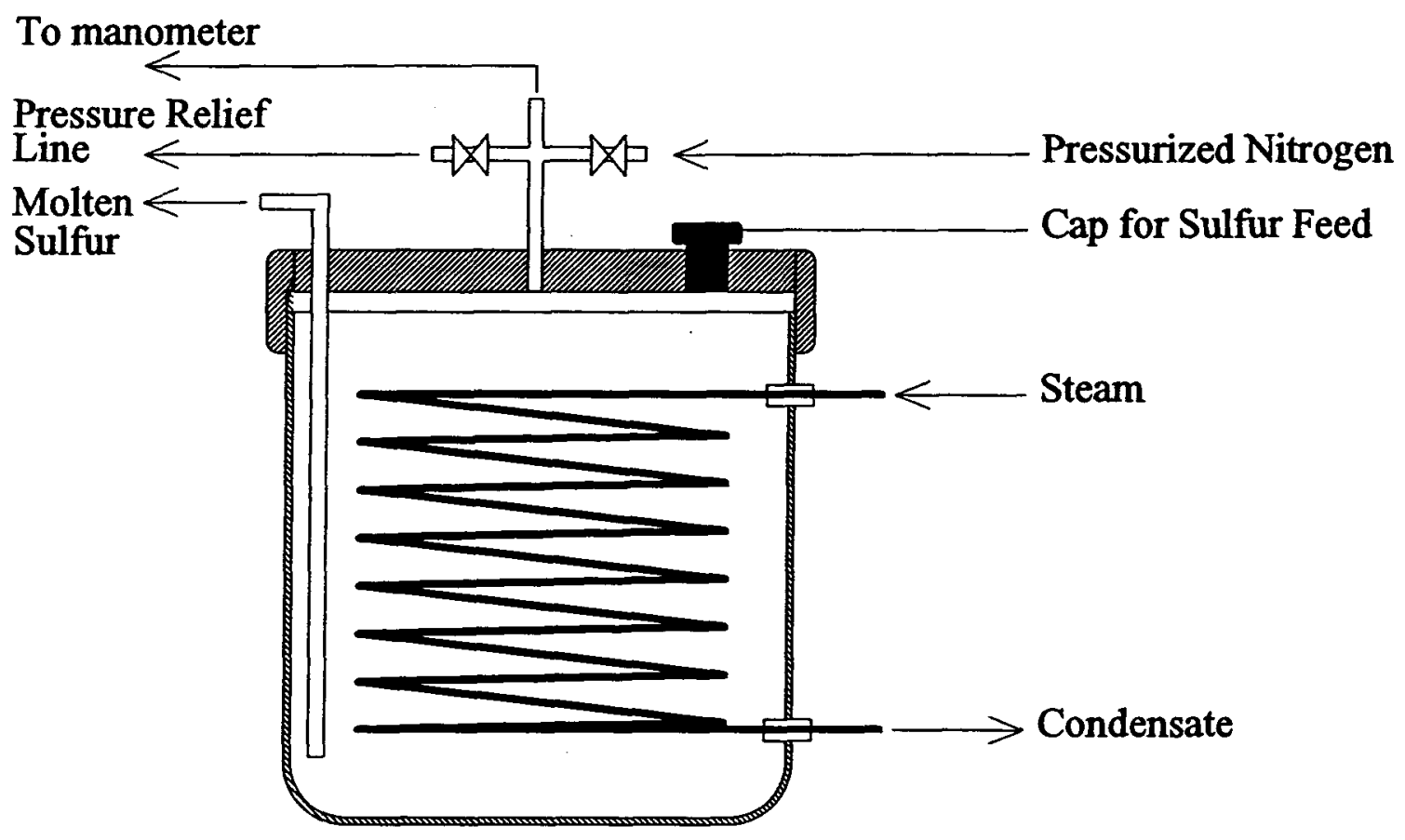

Figure 3.5: Sectional view of $18.9 \mathrm{~L}$ sulfur reservoir.

\subsubsection{Sulfur Filter}

The stainless steel 316 in-line cartridge filter (Rigimesh, manufactured by Pall Canada Ltd.) was located at the mouth of the molten sulfur outlet. The screen size of the filter was $149 \mu \mathrm{m}$ (screen size \#100).

\subsubsection{Sulfur Rotameter}

A standard rotameter tube (Brooks, Model R-6M-25-A) inside a steam heated brass block was used as the sulfur rotameter (designed by Weiss and Meisen, 1983). Two stainless steel pieces on the top and bottom of the brass casing were used to hold the rotameter tube in place and Viton O-rings were used to seal the ends. Two polycarbonate windows with heat resistant gaskets were placed in front and back of the brass block to allow a clear view of the rotameter tube. A set of glass and stainless steel floats was used. The 
specifications of all rotameters are given in Table 3.5, and their calibration curves are presented in Appendix IV.

\subsubsection{Sulfur Line}

The main sulfur line was a $6.4 \mathrm{~mm}$ dia. $316 \mathrm{SS}$ tube enclosed by $13 \mathrm{~mm}$ dia. O.D. TFE tubing overbraided with 304 SS and insulated with fiberglass. Complete steam tracing was not possible at the points where the line joined the melter, rotameter and base of the bed and frequent plugging was observed at the connection to the base of the bed. A fully steam traced connection required a "slip-on" connector as shown in Figure 3.6. This connector was enclosed in a $19 \mathrm{~mm}$ O.D. SS tube for the passage of steam. No sulfur plug-

Table 3.5: Types and capacities of Brooks rotameters used in this work.

\begin{tabular}{|l|c|l|}
\hline Stream & Maximum Capacity & Rotameter \\
\hline Atomizing air & $0.44 \mathrm{~L} / \mathrm{s}$ & $\begin{array}{l}\text { Tube: R-7M-25-1 } \\
\text { Float: Glass }\end{array}$ \\
\hline Spouting air & $23.9 \mathrm{~L} / \mathrm{s}$ & $\begin{array}{l}\text { Tube: } R-12 \mathrm{M}-25-4 \\
\text { Float: } 12-\mathrm{RS}-221\end{array}$ \\
\cline { 2 - 4 } & $42.3 \mathrm{~L} / \mathrm{s}$ & $\begin{array}{l}\text { Tube: R-12M-127-3 } \\
\text { Float: } 12-R S-221\end{array}$ \\
\hline Sulfur & $1.7 \mathrm{~g} / \mathrm{s}$ & $\begin{array}{l}\text { Tube: } R-6 \mathrm{M}-25-1 \\
\text { Float: } \text { glass and stainless steel }\end{array}$ \\
\hline
\end{tabular}

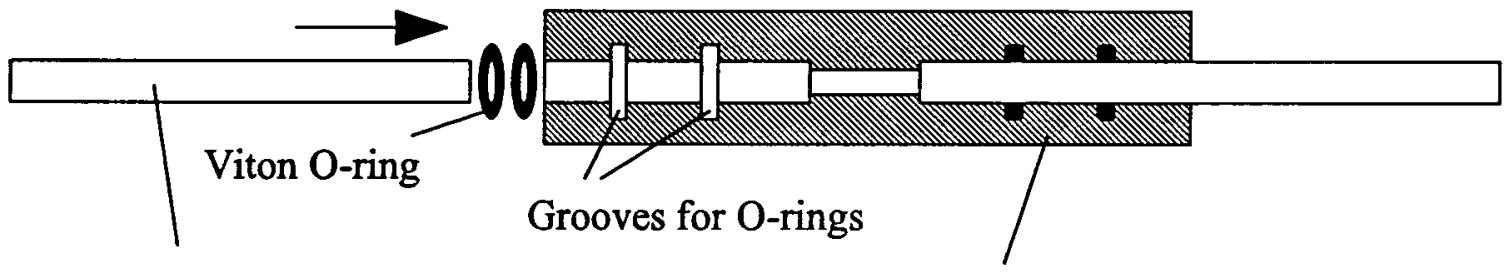

Sulfur line (6.35 mm SS tube) Line connector (9.53 mm SS tube)

Figure 3.6: Sectional view of "slip-on" sulfur line connector. 
ging of the sulfur lines was experienced with this modification.

\subsubsection{Nitrogen Supply}

Industrial grade pressurized nitrogen (typically up to $20 \mathrm{psi}$ ) was used to force sulfur out of the melter. The sulfur flow rate was controlled by the $\mathrm{N}_{2}$ pressure using the regulator on the gas cylinder. The rotameter valve was also used to control the flow rate of the sulfur.

\subsubsection{Nozzle Assembly}

The nozzle assembly consisted of a perforated plate, "steam chamber", and spray nozzle as shown in Figure 3.7. The perforated plate served as a flow straightener and air distributor for the spouting air. The steam chamber kept the sulfur and atomizing air lines at a constant temperature. The atomizing air and sulfur lines were connected to the upper flange and sealed with Viton O-rings.

An "internal-mixing" type pneumatic nozzle was used for spraying sulfur (see Figure 3.7). It consisted of fluid cap \# 40100, air cap \# 1401110 and retainer ring manufactured by Spraying Systems Co. Molten sulfur flowed through the fluid cap that narrowed into a fine tip, before entering the air cap with the atomizing air. Atomizing air entered through three equally spaced holes into the gap between the air cap and the fluid cap. The air and sulfur streams converged just above the nozzle tip thus forming sulfur droplets.

\subsubsection{Urea Feeding Device}

Urea pellets were stored in a $170 \mathrm{~L}$ steel drum with a conical base. The drum was placed $0.75 \mathrm{~m}$ above the spouted bed. From the storage bin, urea pellets fell into a vibrating magnetic feeder (Model F-T01A, manufactured by FMC Corp.) mounted directly under 


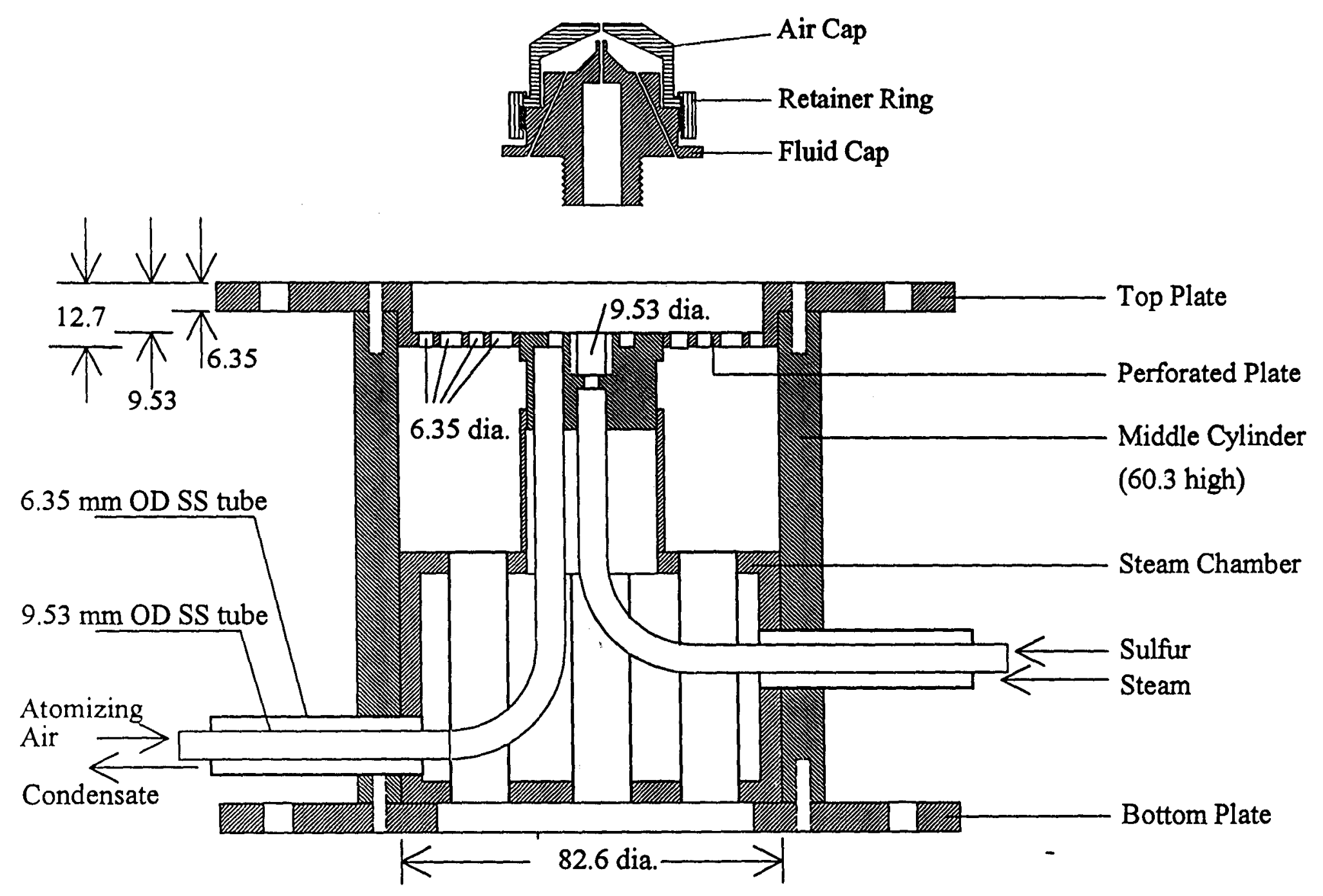

Figure 3.7: Sectional view of nozzle, perforated plate and steam chamber (all dimensions in mm; designed by Meisen et al., 1986). 
neath the bin. The urea feed rate was controlled with an electric controller (Model CSCR 1B, FMC Corp.). The urea was introduced into the bed through a $25 \mathrm{~mm}$ ID Plexiglas tube just above the annulus near the wall and opposite the product withdrawal port. Urea could not be fed below the surface of the annulus due to slow moving bed particles near the wall.

\subsubsection{Product Withdrawal Device}

The product discharged through a column slot and a $25.4 \mathrm{~mm} \mathrm{ID}$ Plexiglas tube. The tube was connected to a PVC flexible plastic hose which directed the SCU into the product storage bin. The discharge mechanism depended on the gravity and air flow into the storage bin from the bed resulting from the pressure difference between the two.

\subsubsection{Product Collector}

The product leaving the spouted bed was collected in a large wooden box $(1.21 \times 0.85 \mathrm{x}$ $0.64 \mathrm{~m}$ high). The box was air sealed with silicone and gaskets to contain any dust. Three holes were drilled through the top board: a $19 \mathrm{~mm}$ dia. hole for the incoming product, a 54 $\mathrm{mm}$ dia. hole for air exhaust, and a $0.3 \mathrm{~m}$ dia. hole for cleaning the box. The $0.3 \mathrm{~m}$ hole was covered with a $13 \mathrm{~mm}$ thick Plexiglas lid held in place by attaching the cover to a 0.05 $x 0.33 \mathrm{~m}$ Plexiglas board inside the box. A $25 \mathrm{~mm}$ dia. hole was also drilled through the bottom board to empty the box.

\subsubsection{Dust Collector}

Urea and sulfur fines elutriated from the top of the spouted bed passed through a flexible exhaust hose into a water scrubber (see Figure 3.2). The treated air was then vented directly into the laboratory exhaust system. A nylon mosquito mesh (approximately $1 \mathrm{~mm}$ mesh size) was placed on top of the bed in the exhaust air line to prevent the bed particles from leaving the bed. 


\subsubsection{Air, Steam, and Water Supplies}

All air lines were connected to the laboratory compressed air supply (maximum pressure of $510 \mathrm{kPa}$ ). Instrument air (maximum pressure of $650 \mathrm{kPa}$ ) was used for sulfur atomization to provide cleaner air. Rotameters were used for all flow measurements. The type and capacities of the rotameters are given in Table 3.5.

The spouting air was heated by a steam heater and a $3 \mathrm{~kW}$ electric heater. The atomizing air was heated by a flexible electric heating tape (Type silicone rubber, 312 Watts; manufactured by Thermolyne Corp.) wrapped around the air line. The electric heater was controlled by a proportional-integral controller supplied by Omega Engineering Inc. (Model No. 49J, range of 0 to $200^{\circ} \mathrm{C}$ ). The temperatures were monitored with iron-constantan thermocouples.

Steam was generated by a $30 \mathrm{~kW}$, three phase, "Electro-Steam" boiler (Type F-10; manufactured by Fulton Ltd.) capable of steam flows up to $48 \mathrm{~kg} / \mathrm{h}$ at $720 \mathrm{kPa}$. The operating pressure was set at $650 \mathrm{kPa}$ for all runs in this study. The pressure downstream from the boiler typically fell into the range of 580 to $620 \mathrm{kPa}$. All steam traps discharged into a common atmospheric header that drained into the main sewer system.

Cold tap water was used in the water scrubber.

\subsection{Apparatus for Hydrodynamics Study}

The modifications to the spouted beds for the hydrodynamics study included replacing the stainless steel caps with $1 / 2^{\prime \prime}(13 \mathrm{~mm})$ plywood caps. The new caps were slotted to allow a sampling tube to enter from the top of the bed, and the tube could be positioned at any radial position. The modified cap is shown in Figure 3.8. 


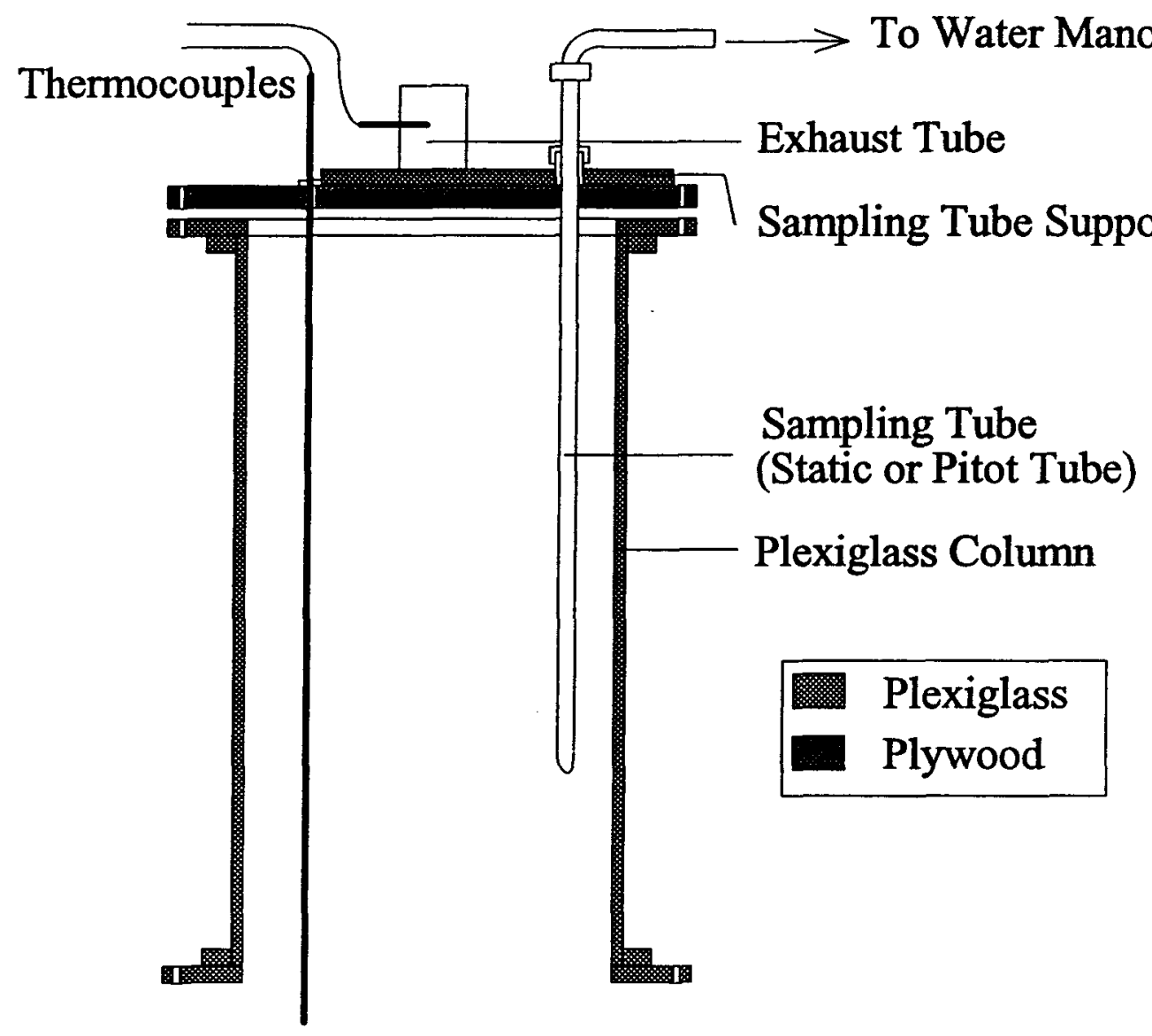

Figure 3.8: Modifications to the top of the spouted bed (i.e., see Figure 3.3) for hydrodynamics study.

A static pressure probe (see Figure 3.9) and an S-type pitot tube (see Figure 3.10) were used to measure the static pressure in the annulus and the fluid velocity in the spout, respectively. The tubes were secured to the top of the bed by a $64 \times 230 \mathrm{~mm}$ Plexiglas sampling tube support and Swagelok fittings.

\subsection{Apparatus for Spray Study}

Sulfur spray droplets produced by the atomizing nozzle described in Section 3.2.3 were captured in an air-sealed spray box. The box was designed to be placed immediately above the nozzle assembly (see Section 3.2 .3 ). The nozzle assembly was not modified for this study. The two main components of the spray box are the box and the spray sampler. 


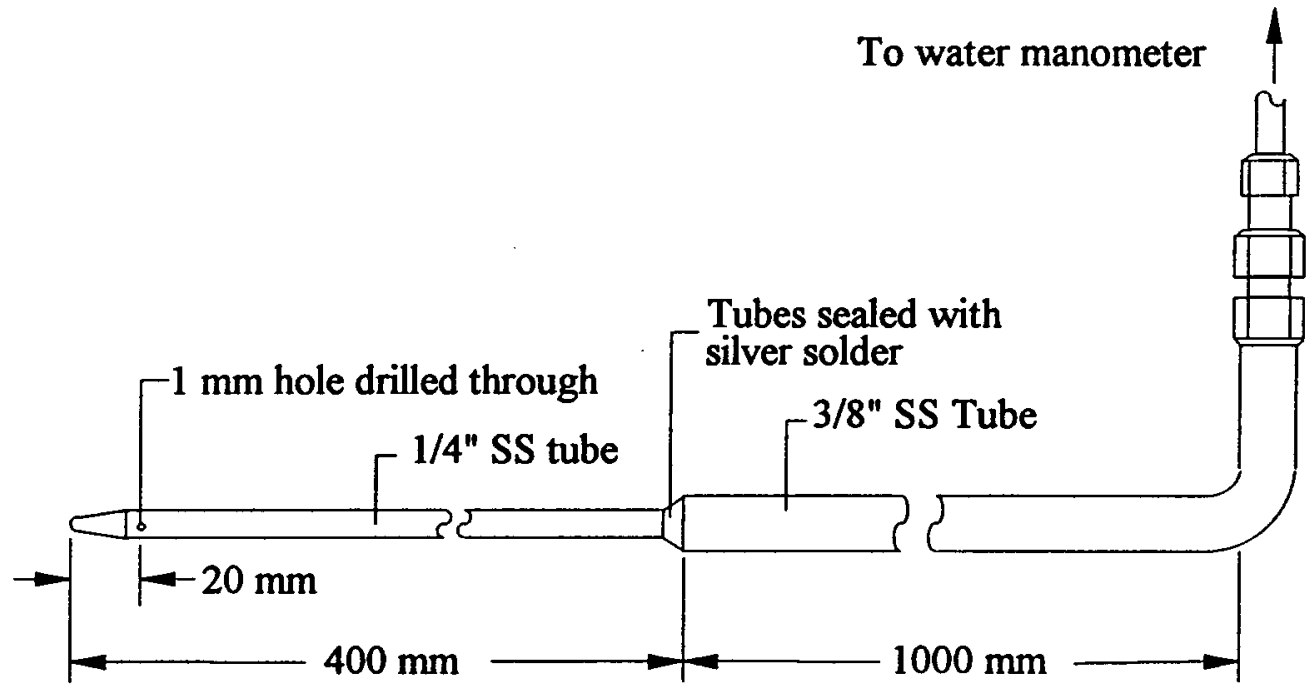

Figure 3.9: Schematic diagram of static pressure probe.

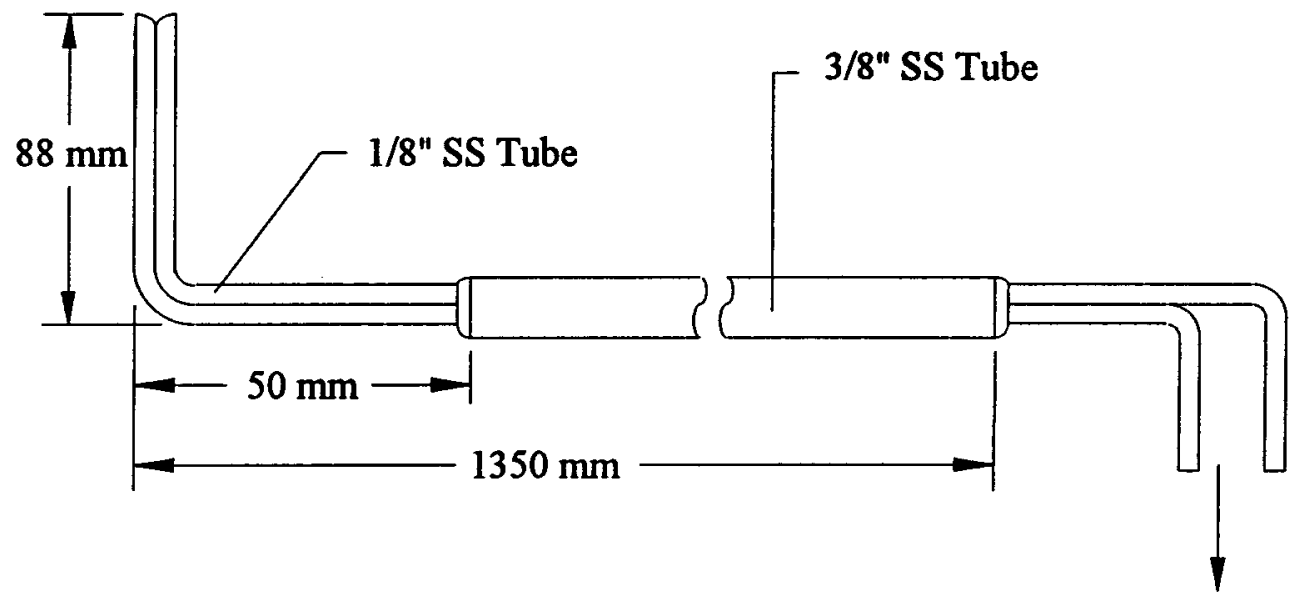

To water manometer

Figure 3.10: Schematic diagram of S-type pitot tube (1/8" tubes and 3/8" tube are held in place with silver solder).

\subsubsection{Spray Box}

The design of the $0.61 \times 0.61 \times 1.22 \mathrm{~m}$ spray box used to sample sulfur droplets is shown in Figure 3.11. The box was constructed of a rigid 1/2" $(13 \mathrm{~mm})$ plywood base, back and side walls with $6.4 \mathrm{~mm}$ Plexiglas viewing panels and a $3.2 \mathrm{~mm}$ aluminum cap. Side walls were slotted $(57 \mathrm{~mm})$ for the insertion of the sampling tube 0.15 to $0.3 \mathrm{~m}$ from the base of 


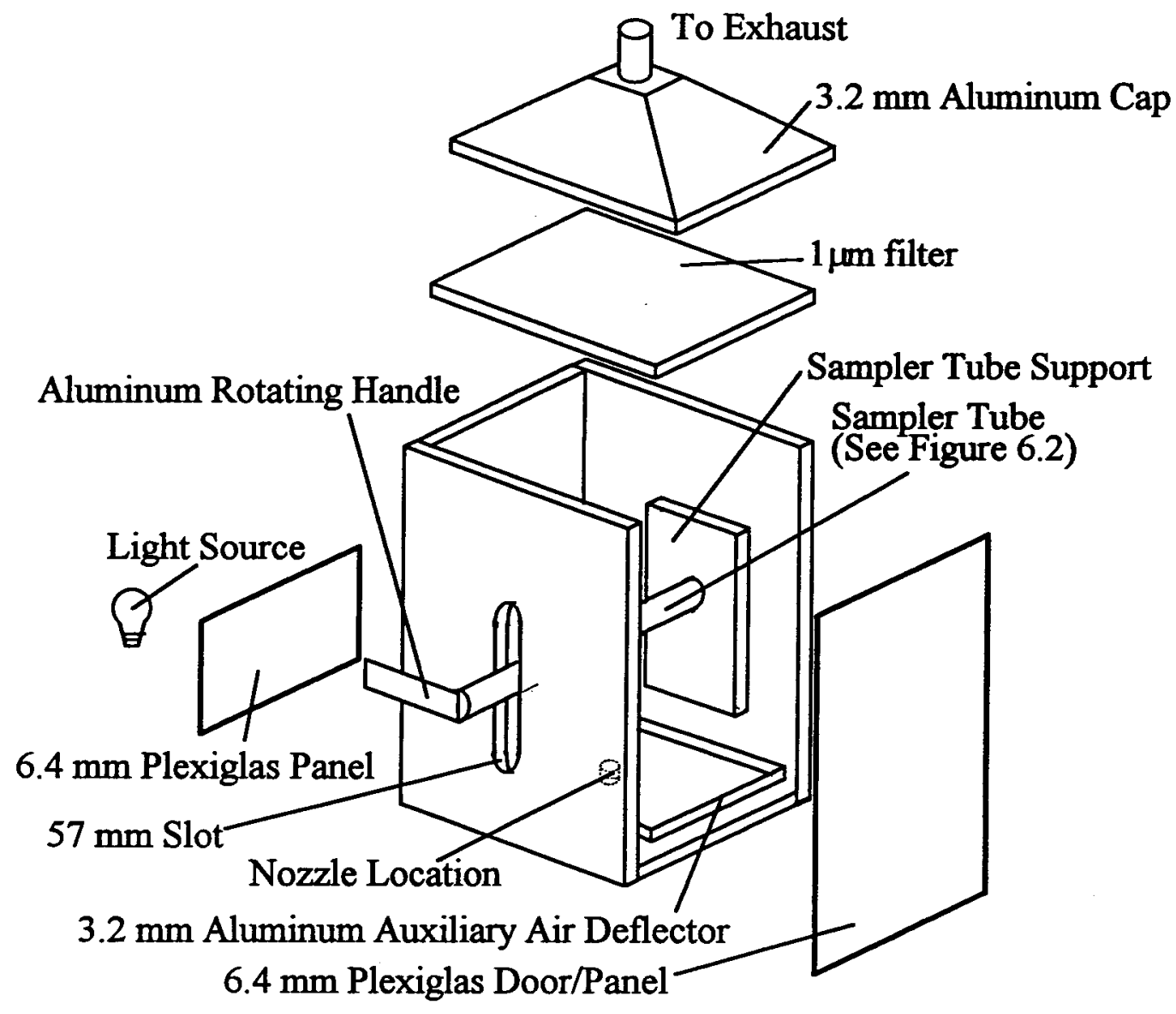

Figure 3.11: Spray box assembly.

the nozzle, and the slots were sealed once the sampling tube was placed inside the box. A light source (100 W lamp) was placed behind the box to improve the visibility of the spray. An aluminum plate was placed over the base of the bed to force auxiliary air (normally used as the spouting air in spouted bed operations) along the walls to reduce spray depositions on the walls and to improve the spray's visibility. The spent air was cleaned using an air filter ( $1 \mu \mathrm{m}$ pore size; type: Blue \#100 SYN; manufactured by B.C. Air Filter Ltd.) before being vented into the laboratory exhaust system.

\subsubsection{Spray Sampler}

The spray sampler consisted of an outer aluminum shell and a glass slide support made of a $51 \mathrm{~mm}$ O.D. aluminum rod as shown in Figure 3.12. The rod was machined to hold 
Protective Shell: $51 \mathrm{~mm}$ I.D. SS tube

Cross-Sectional

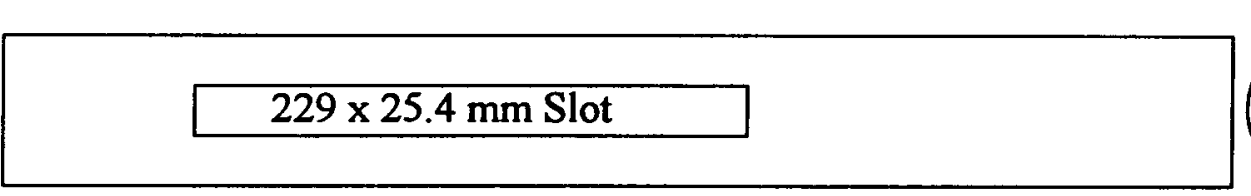

View

Glass Slide Support: $51 \mathrm{~mm}$ O.D. Aluminum Rod
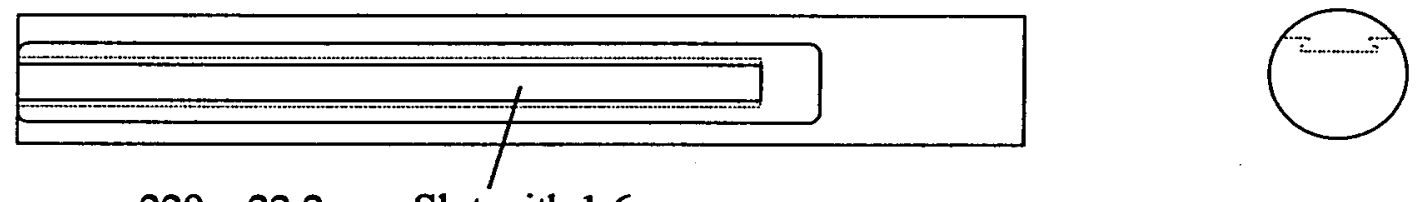

$229 \times 22.2 \mathrm{~mm}$ Slot with $1.6 \mathrm{~mm}$ grooves

Figure 3.12: Simplified drawing of the rotating sulfur droplet sampler.

three sample cells ( $25.4 \times 76.2 \mathrm{~mm}$ glass slides). The sampling unit could be withdrawn through the side of the box to replace the glass slides. The outer shell was secured by rings fitted to the sampler tube supports, and could be rotated using a rotating handle outside the box.

\subsection{Coating Procedures}

The operating procedures for the production of sulfur coated urea in a spouted bed consists of three steps: "start up", "coating", and "shut down".

\subsubsection{Start Up}

Due to the lengthy melting time of sulfur, the sulfur was typically melted overnight. This step required the following procedure: the boiler (steam generator) was turned on with all valves connected to the coating facility closed, the melter was filled with solid sulfur, the water flow to the scrubber was turned on (the water from the scrubber served to reduce loud crackling noises created by the water traps), and the main power supply and the temperature readout were switched on to monitor the temperatures at various points of the 
equipment. Once the pressure in the boiler reached $80 \mathrm{psig}(650 \mathrm{kPa})$, the steam was introduced to the melter by opening the appropriate valves.

In the meantime, the urea hopper was filled and the spray nozzle was placed in position. The nozzle was checked to ensure there were no plugs.

\subsubsection{Coating}

The coating operation required the following steps: (i) the spouted bed was placed in the "coating" position with the shutter open; (ii) atomizing air, spouting air, and their respective heaters were turned on; (iii) the urea feeder was turned on after the air temperatures reached 80 and $160{ }^{\circ} \mathrm{C}$ for the spouting and atomizing air, respectively; (iv) the bed was left spouting with the withdrawal outlet open until steady state temperatures were observed; (v) the nitrogen supply (set at approximately 5 psig) was opened to the sulfur melter, and the valve attached to the rotameter was opened (an electric heating gun was frequently used to unblock the valve); (vi) the nitrogen pressure regulator and the rotameter valve were adjusted to set the sulfur flow rate to the desired values; (vii) steady state (indicated by a constant bed temperature and fountain height) was usually reached after ten minutes of coating for a given set of operating conditions, and product samples were collected; (viii) when the experiment was complete, the nitrogen supply valve and the urea feeder were turned off; (ix) the pressure was relieved from the melter by opening the pressure relief valve and the sulfur flow valve was shut off after the sulfur had flowed back into the melter; $(x)$ the air heaters were turned off, the atomizing air was reduced to minimum, and the shutter and the spouting air valve were closed simultaneously, (xi) the spouted bed was detached from its base, and the bed particles were unloaded.

The operating bed temperature $\left(T_{b}\right)$ was taken as the steady state bed temperature after the sulfur was injected into the bed. This temperature was typically 5 to $10^{\circ} \mathrm{C}$ higher than the temperature before the sulfur injection. 
To take a sample of the product, the flexible hose in the product withdrawal line was disconnected from the product storage bin and attached to a small container. A sudden surge of particles resulted from the pressure difference between the bed and atmosphere, and approximately a minute was allowed for the bed to return to steady state conditions before the products were sampled. The sampling time was also recorded to determine the production rate.

All samples at different operating conditions were collected by repeating the above procedure for consistency, i.e. the bed was emptied before starting a new run.

\subsubsection{Shut Down and Clean-up}

The procedure for shutting down was as follows: the spray nozzle was removed, inspected for plugging, and soaked in $50 \% \mathrm{NaOH}$ solution, all sources of steam, power, water and air were turned off, and the column was cleaned.

\subsection{Procedures for Hydrodynamics Study}

\subsubsection{Minimum Spouting Velocity, $U_{m s}$}

The minimum spouting velocity was found by spouting the particles and then gradually reducing the air flow rate until spouting ceased. $U_{m s}$ was calculated from the total volumetric air flow rate, $Q_{m s}$ just before cessation of spouting, i.e.,

$$
U_{m s}=4 Q_{m s} / \pi D^{2}
$$

where $D$ denotes the diameter of the cylindrical portion of the bed. It should be noted that the total air flow rate $\left(Q_{t}\right)$ is the sum of the spouting $\left(Q_{s}\right)$ and atomizing $\left(Q_{a}\right)$ air flow rates. The errors in the measurements, calculated from the rotameter scale readings and from repeat measurements, were typically less than $\pm 5 \%$. 


\subsubsection{Voidage of Loosely Packed Bed, $\varepsilon_{m f}$}

The voidage $\left(\varepsilon_{m f}\right)$ was determined by quickly inverting and re-inverting a glass cylinder ( $0.1 \mathrm{~m}$ dia. by $0.6 \mathrm{~m}$ high) filled approximately one-third with particles. The voidage was calculated from the following equation:

$$
\varepsilon_{m f}=\frac{V_{b}-m_{p} / \rho_{p}}{V_{b}}
$$

\subsubsection{Mean Particle Diameter, $d_{p}$, and Sphericity, $\phi_{s}$}

The mean diameter was found by sieving the particles using \#5 to \#14 Tyler mesh screens and the equation

$$
d_{p}=1 / \sum_{i} x_{i} / d_{p i}
$$

where $x_{i}$ denotes the mass fraction of particles with diameter $d_{p i}$

With the exception of polystyrene, all particles were nearly spherical (see Plates 3.1 - 3.5). The sphericity of the elliptical-cylindrical polystyrene particles (see Plate 3.6) was determined by measuring the principal dimensions $(\sim 2.2 \times 3.1 \times 3.3 \mathrm{~mm})$ of a representative sample containing over 80 particles. Other particles were assumed to be perfect spheres, i.e., $\phi_{s}=1$.

\subsubsection{Diameter of Inlet Orifice, $d_{i}$}

The orifice is partly obstructed by the presence of the atomizing air nozzle (see Figure 3.13). The effective orifice diameter $\left(d_{i}\right)$ is therefore calculated from

$$
d_{i}^{2}=d_{o}^{2}-d_{a}^{2}
$$

and $\quad d_{o}^{2}=4 A_{o} / \pi$

where $A_{o}, d_{o}$, and $d_{a}$ denote the area of the orifice opening, the area equivalent diameter of the orifice opening and the diameter of atomizing air nozzle, respectively. 


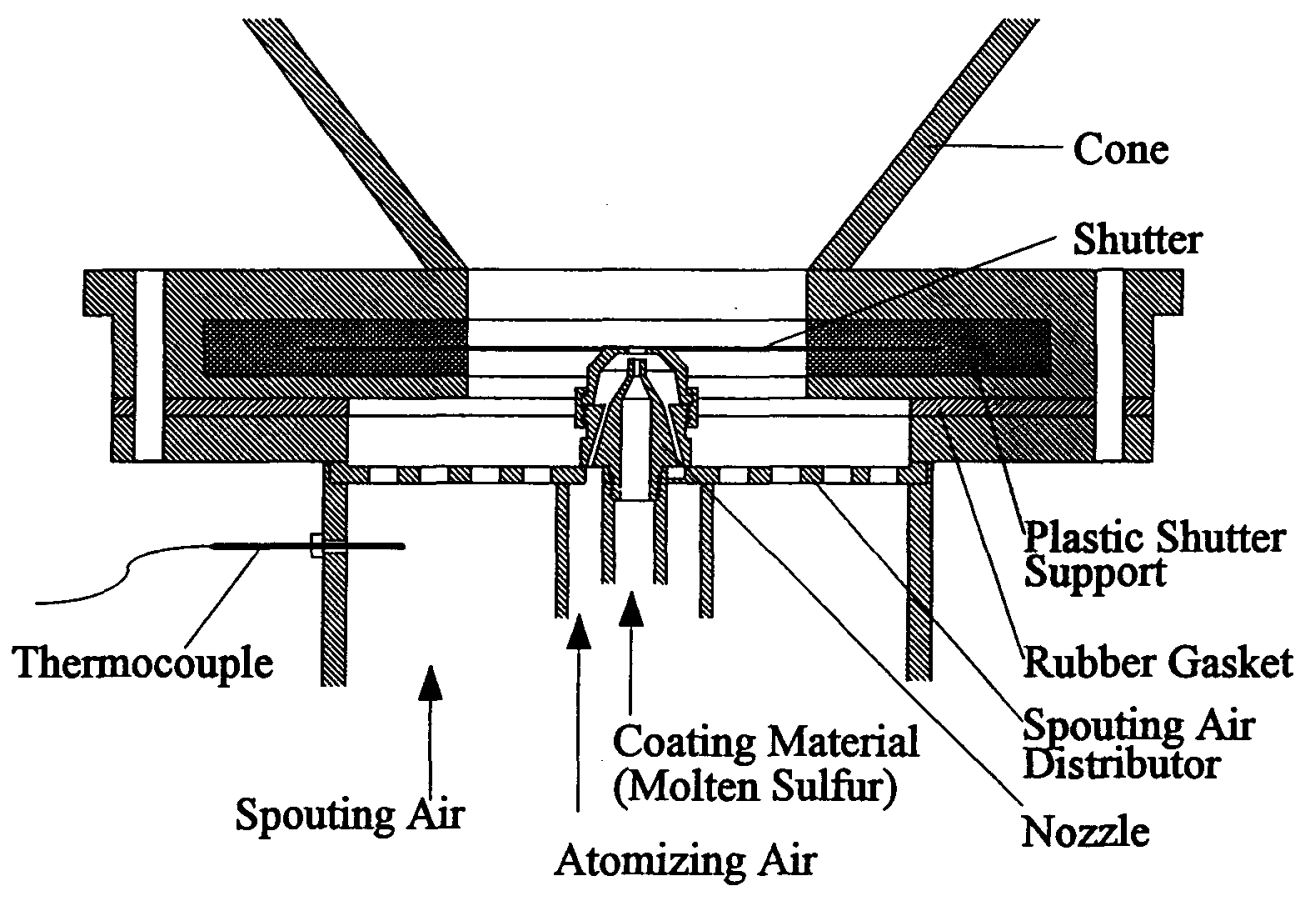

Figure 3.13: Sectional view of atomizing nozzle and inlet air assembly (not drawn to scale).

\subsubsection{Static Pressure in Annulus}

The static pressure was determined by using the probe shown in Figure 3.9. The probe was inserted into the top of the bed and moved horizontally and vertically to the desired location. The pressure readings were taken with a water manometer, one leg of which was open to the atmosphere.

\subsubsection{Air Velocity in the Spout, $u_{s}$}

The air velocity in the spout was determined by using the S-type Pitot tube shown in Figure 3.10 and connected to a water manometer. The tube was inserted into the top of the bed and lowered to the desired position. It was then moved radially until the maximum pressure difference was noted. This corresponded to the axis of the spout and was used to 
calculate $u_{s}$. (Therefore, the measured $u_{s}$ corresponds to the maximum velocity in the spout.)

The tube was calibrated against an ASME (1971) standard static pitot tube to $40 \mathrm{~m} / \mathrm{s}$ in a wind tunnel ( $0.30 \times 0.41 \mathrm{~m}$ by $3.7 \mathrm{~m}$ long): The standard pitot tube coefficient $\left(C_{\mathrm{o}}\right)$ was then determined from the following equation:

$$
u=C_{o} \sqrt{2 \Delta P / \rho}
$$

The calibration curve is shown in Appendix IV.

\subsubsection{Radial Velocity Profile at the Base of the Bed}

In the absence of the bed particles, the radial air velocity distribution $10 \mathrm{~mm}$ above the shutter was measured with a static pitot tube connected to a water manometer. The tube consisted of a $1 / 16^{\prime \prime}(1.6 \mathrm{~mm})$ stainless steel inner tube and a $1 / 8^{\prime \prime}(3.2 \mathrm{~mm})$ stainless steel outer tube. The calibration curve and coefficient $\left(C_{o}\right)$ for the static pitot tube are provided in Appendix IV.

\subsection{Procedures for Spray Studies}

\subsubsection{Operating Limits of Spray Nozzle}

The operating envelope of the spray nozzle used in this work was investigated by determining the upper and lower limits of the atomization velocity. The lower limit or the minimum atomization velocity $\left(u_{m a}\right)$ was determined by gradually reducing the atomizing air flow rate from a fully atomized state until no atomization was observed. The velocity was calculated from the superficial air flow rate referenced to the nozzle orifice $\left(d_{a t}\right)$. The upper limit or the minimum pulsating velocity $\left(u_{m p}\right)$ was determined by gradually increasing the atomizing air flow rate until inconsistent atomization (indicated by flickering of the spray) was observed. 


\subsubsection{Spray Drop Size Measurements}

All samples of sulfur droplets were collected $0.2 \mathrm{~m}$ above the nozzle. At this distance, the sprays were fully developed and the spray density was low enough for easy sampling. The sampling time and the slot size on the protective tube were also varied to obtain low spray density (effects of sample time and the slot size are discussed in Chapter 5).

The procedure for collecting the spray samples was as follows: (i) the glass slides were placed on the slide holder (see Figure 3.12); (ii) the holder was placed in the spray box; (iii) the spray was turned on at desired sulfur and atomizing air flow rates; (iv) the outer shell was rotated using the rotating handle to expose the glass slides to the spray; (v) the glass slides were removed and the procedure was repeated for a new operating condition.

The sulfur drops on the glass slides were analyzed under a microscope attached to a LIETZ TAS Image Analyzer available in the Department of Metals and Materials Engineering at UBC. At least ten frames of size 750 by $750 \mu \mathrm{m}$ were analyzed to obtain approximately 100 droplets for each run. Depending on the operating condition, most drop samples contained more than 100 drops in ten frames. Non-spherical particles were not included in the analysis.

\subsection{Product Quality Analysis}

\subsubsection{Sulfur Content}

The total sulfur deposited on the urea particles was determined by two methods. One method involved mass balance calculations based on the urea and sulfur feed rates; negligible loss of sulfur and urea fines was assumed. The other method was more direct and is referred to as the crush test. In the latter method, either the sulfur or urea could be analyzed to determine the sulfur content. The former was used in this work to obtain the mean sulfur content of the product. 
The procedure for the crush test was as follows: a weighted sample (approximately $20 \mathrm{~g}$ ) of sulfur coated urea was ground in a crucible with $10 \mathrm{~mL}$ of water to obtain a fine slurry (the exposed urea readily dissolved). The slurry was washed into a filter ( $1 \mu \mathrm{m}$ pores) with excess water. The filtered sulfur was left over night to dry and the dried sulfur was weighed the following day. The results could be reproduced to approximately $\pm 1 \%$ for batch products, and $\pm 3 \%$ for continuous products.

\subsubsection{Particle Sulfur Content}

Up to one hundred individual SCU particles, randomly selected, were analyzed to determine the sulfur content distribution for each product. The analysis of sulfur (as described in Section 3.8.1) was difficult due to the relatively small mass of sulfur on each particle. Consequently, the urea content was analyzed to determine the sulfur content of each particle. Each particle was weighed, placed in a $0.5 \mathrm{~mL}$ vial and crushed (using a paper clip) in $0.5 \mathrm{~mL}$ of distilled water. The solution was capped and left overnight to ensure the complete dissolution of urea. The refractive index of the solution was measured with an Abbe refractometer (Model JB7150, Bausch and Lomb Optical Co.). The urea concentration was determined from the refractometer calibration curve (see Appendix IV). The urea content in the solution could be calculated once its concentration was known. The sulfur content was determined by the difference between the urea and the total particle weights.

The error due to the particle weight measurement (typically 5 to $30 \mathrm{mg}$ ) was as large as \pm $20 \%$ while the error due to the urea content measurement using the refractometer was even higher (up to $\pm 25 \%$ ) for $5 \mathrm{mg}$ particle. However, the errors for particles bigger than $5 \mathrm{mg}$ were typically less than \pm 10 and $12 \%$, respectively. 


\subsubsection{Seven Day Dissolution Test}

The standard seven day test developed by TVA was used, i.e. $50 \mathrm{~g}$ of sulfur coated urea and $250 \mathrm{~mL}$ of distilled water were placed in a capped jar for seven days at $37.8^{\circ} \mathrm{C}$ in an incubator. The solution was carefully poured out and stirred. A sample of the solution was analyzed under the refractometer for the urea concentration as mentioned in the previous section (Section 3.8.2). The seven day dissolution rate $\left(U_{D m}\right)$ was determined as

$$
U_{D m}=\frac{\text { weight of urea dissolved }}{\text { weight of urea in sample }} \times 100 \%
$$

The results could be reproduced to approximately $\pm 2 \%$ for batch products, and to less than $\pm 5 \%$ for continuous products. 


\section{Chapter 4. Mathematical Models}

Mathematical models describing the spouted bed coating process for the production of sulfur coated urea (SCU) are developed in this chapter. The first section deals with simple models based on assumptions used for previously published coating models (see Section 2.2). The simple models are then improved by progressively relaxing various assumptions to determine how they limit these models. Subsequent sections deal with the development of a "rigorous" model and programming strategy.

\subsection{Simple Models}

The objectives of developing simple models are to test some of the assumptions used in the previously mentioned models in the literature, to determine the limitations of the models, and to establish the need to develop a more comprehensive model. The simple models can provide quick estimates of the coating performances of a spouted bed, which may be useful in preliminary plant design.

\subsubsection{Model I: Residence Time Model}

In developing the first model, two principal assumptions are made: perfect mixing of the bed particles and uniform sulfur spray concentration throughout the bed. In addition, negligible coating (sulfur spray) is assumed lost. A schematic diagram of Model $I$ is shown in Figure 4.1. The tracer tests conducted by previous investigators (Becker and Sallans, 1961; Kugo et al., 1965; Barton et al., 1968) on spouted beds indicated that the residence time distribution of the bed particles approximated that of a perfectly mixed vessel. The residence time distribution, $F\{t\}$, for bed particles leaving a perfectly mixed vessel is given by:

$$
F\{t\}=1-e^{-t / t}
$$




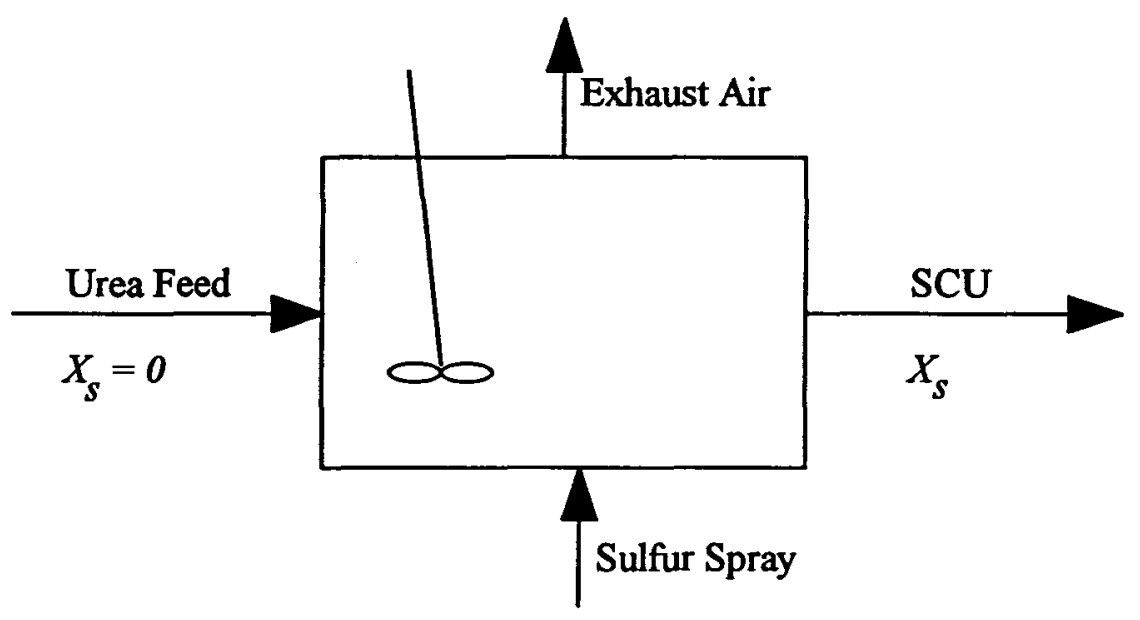

Figure 4.1: Model I - perfectly mixed system.

$F\{t\}$ may be regarded as the probability that a particle entering the bed at time zero leaves after time $\leq t$.

In Model I, the amount of coating (or sulfur), $m_{s}$, on a particle leaving the bed is assumed to be directly proportional to its residence time in the bed, i.e.,

$$
m_{s} \propto t
$$

This assumption is consistent with assuming that a uniform spray concentration exists throughout the bed. It is convenient to express the particle coat distribution in terms of the sulfur content of the particle $\left(X_{s}\right)$ rather than $m_{s}$, i.e.,

$$
X_{s}=\frac{m_{s}}{m_{s}+m_{u}}
$$

where $m_{u}$ denotes the mass of urea in a coated particle. Assuming $m_{u}$ is the same for all particles, Equations (4.2) and (4.3) may be combined to give

$$
t / \bar{t}=\frac{X_{s}}{\overline{X_{s}}} \frac{1-\overline{X_{s}}}{1-X_{s}}
$$

Equation (4.1) may now be rewritten in terms of $X_{s}$ as well: 


$$
G\left\{X_{s}\right\}=1-\exp \left(-\frac{X_{s}}{\overline{X_{s}}} \frac{1-\overline{X_{s}}}{1-X_{s}}\right)
$$

where $G\left\{X_{s}\right\}$ denotes the probability that a particle has a sulfur content less than or equal to $X_{s}$. Only the average sulfur content of the product $\left(\overline{X_{s}}\right)$ must be known to determine the sulfur content distribution of the product. Typical results from this equation are plotted in Figure $4.2(n=1)$.

The correlation given by Barton et al. (1968) showed that the particle residence time distribution in the spouted bed deviated from that in the perfectly mixed bed, i.e.,

$$
F\{t\}=1.026-0.92 \exp (-1.087(t / \bar{t}-0.1))
$$

The results were explained by using mixed-flow models where $8-10 \%$ of the total bed volume in the spouted bed was in plug flow (Quinlan and Ratcliffe, 1970). Chatterjee (1970)

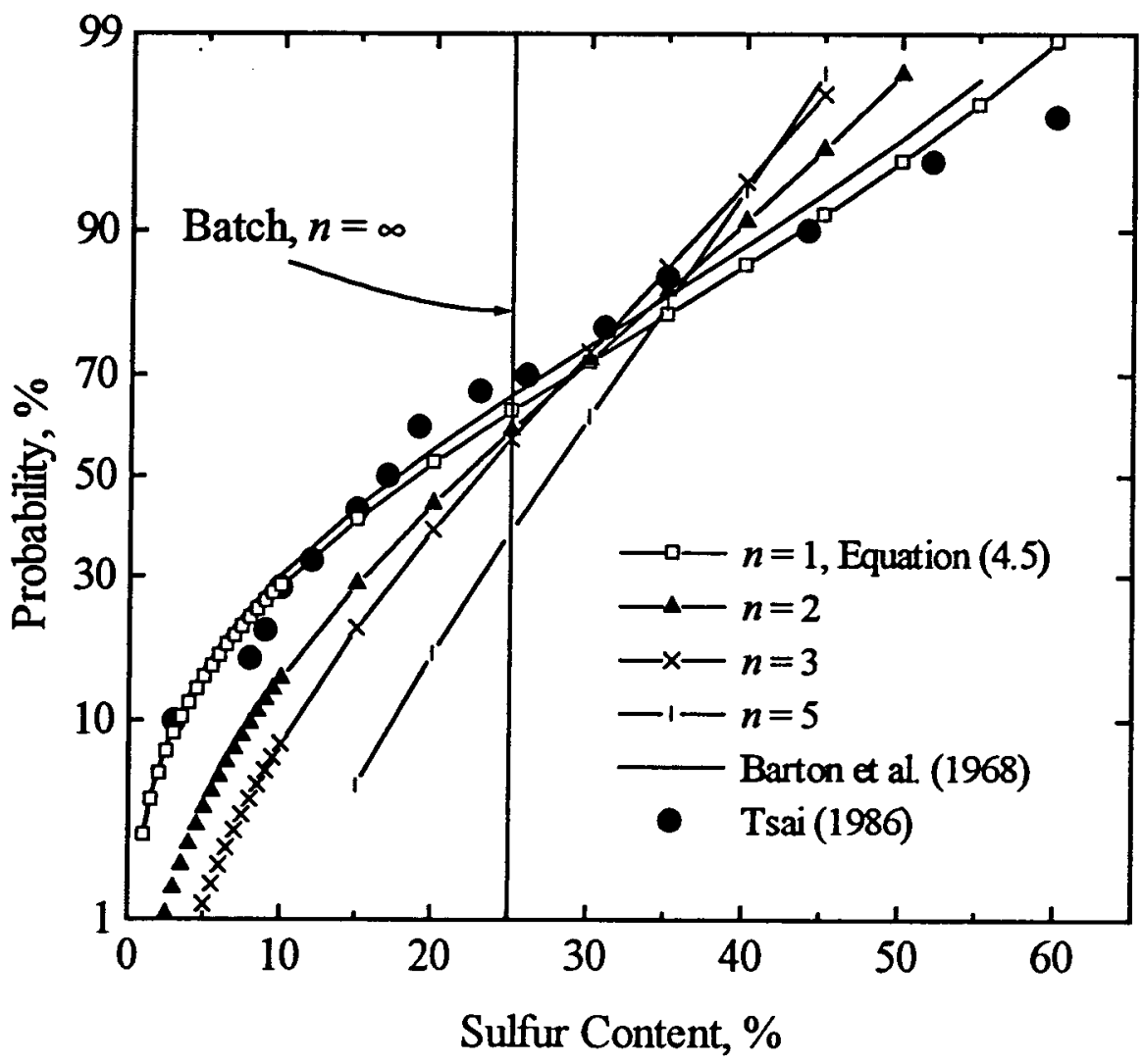

Figure 4.2: Prediction of Model I using different numbers of continuously stirred tanks in series. 
interpreted the residence time distributions in terms of two continuously stirred tanks (CST). The results based on these models are also shown in Figure 4.2. The results of CST's in series were calculated from the following expression (Hill, 1977):

$$
F\{t\}=1-e^{-n t i t}\left[1+\frac{n t}{\bar{t}}+\frac{1}{2 !}\left(\frac{n t}{\bar{t}}\right)^{2}+\cdots+\frac{1}{(n-1) !}\left(\frac{n t}{\bar{t}}\right)^{n-1}\right]
$$

Note that, to obtain Figure $4.2, t / \bar{t}$ in all cases was replaced with the relationship given by Equation (4.4). Batch results for a coating time $\bar{t}$ are also given in Figure 4.2 for comparison.

Tsai's coating distribution data are shown in Figure 4.2 and are fairly well represented in the mid-range of sulfur concentrations by Equation (4.5), except that most of the experimental data are slightly under predicted. In the same range, some improvement was observed with the model given by Barton et al. (1968). Models based on several CST's deviate further from the experimental data.

The presence of significant fractions of uncoated urea particles produced in the continuous process and the particle sulfur content distribution found in the batch product (see Figure 4.3) cannot be predicted by Model I. This leads to the conclusion that the assumption of a uniform spray concentration in the bed is not valid for spouted bed coaters.

\subsubsection{Model II: Simple Spray Zone Model}

The aforementioned deficiencies of Model I suggest the presence of a limited spray zone which some particles do not enter before leaving the spouted bed. In order to incorporate such a spray zone, Model II was created on the premise that the bed could be divided into three regions: spout, annulus and fountain. The spray zone is located in the lower region of the spout, and the amount of coat a particle receives per pass through the spray zone is fixed, i.e., the same amount of coat is applied on all particles regardless of the positions at 


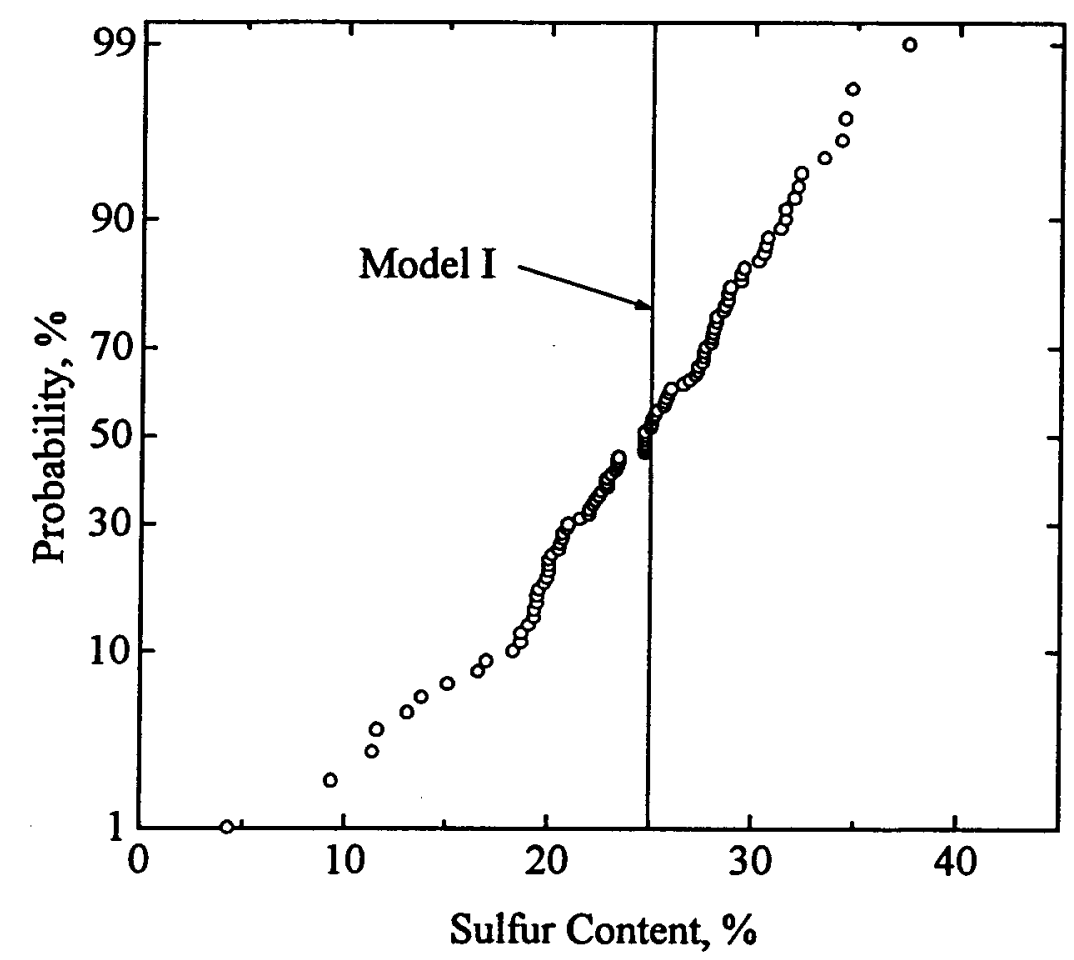

Figure 4.3: Prediction of Model I (Equation (4.7), $n=\infty$ ) and batch product from the current work $\left(\bar{X}_{s}=0.25, W_{s}=4.1 \mathrm{~g} / \mathrm{s}, d_{i}=28.2 \mathrm{~mm}, H=0.25 \mathrm{~m}, D=0.24\right.$ $\mathrm{m}, Q_{a}=0.57 \mathrm{~L} / \mathrm{s}$ and $T_{b}=75^{\circ} \mathrm{C}$ ).

which they enter the spray zone. It is presumed that urea is introduced directly into the annulus and that SCU is withdrawn from the fountain. Other assumptions are listed in Table 4.1. The system described by Model II is illustrated in Figure 4.4.

The mathematical derivation of the model equations is accomplished by examining the probability of particles receiving certain amounts of coating by the time they leave the bed. For example, the total probability of particles receiving no coat before exiting the bed $\left(P_{O_{c}}\right)$ is the sum of the probability of the particles by-passing the spray zone $\left(x_{b}\right)$ combined with the probability of leaving the bed $\left(x_{e}\right)$, i.e.,

$$
\begin{aligned}
P_{0 c} & =x_{e} x_{b}+x_{e} x_{r} x_{b}^{2}+x_{e} x_{r}^{2} x_{b}^{3}+\cdots x_{e} x_{r}^{n-1} x_{b}^{n}+\cdots \\
& =\sum_{n=1}^{\infty} x_{e} x_{r}^{n-1} x_{b}^{n}
\end{aligned}
$$


Table 4.1: Assumptions used in simple models.

\begin{tabular}{|c|c|c|c|c|c|}
\hline & \multicolumn{3}{|c|}{ Perfect mixing } & \multicolumn{2}{c|}{ Spray zone } \\
\cline { 2 - 6 } & Spout & Annulus & Fountain & Location & $\begin{array}{c}\text { Sulfur drop- } \\
\text { let concen- } \\
\text { tration }\end{array}$ \\
\hline Model I & Yes & Yes & Yes & All & Uniform \\
\hline Model II & No & No & Yes & Lower spout & Fixed \\
\hline Model III & No & No & Yes & Lower spout & Variable \\
\hline
\end{tabular}

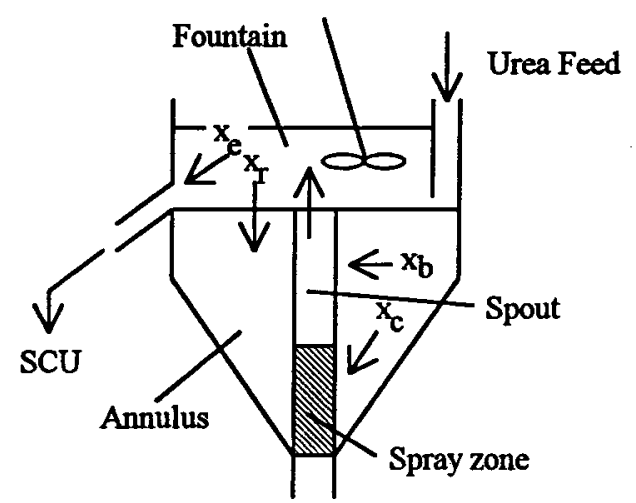

a). Spouted Bed

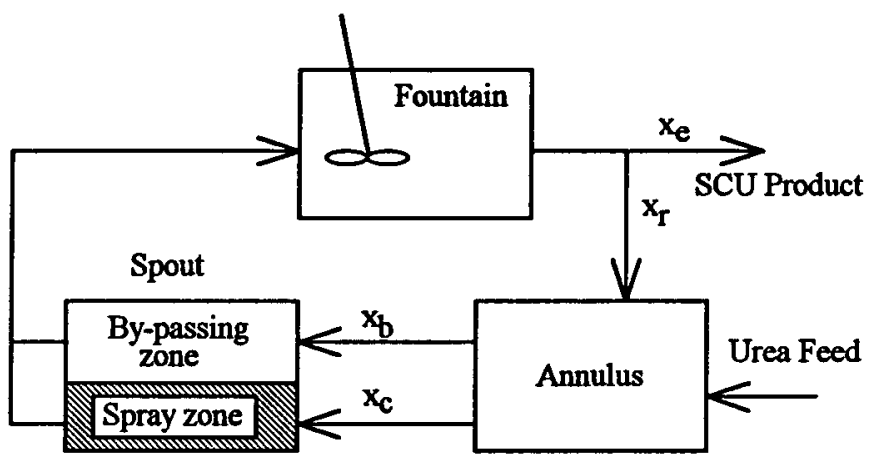

b). Simplified Spouted Bed

Figure 4.4: System described by Model II.

where $x_{r}=1-x_{e}$ denotes the probability of a particle not exiting from the bed when it leaves the fountain. The terms on the right hand side of Equation (4.8) represent the probabilities of particles not receiving any coat after one to an infinite number of cycles. Inspection of the infinite series formulas given in Table 4.2 reveals that Equation (4.8) reduces to:

$$
P_{0 c}=x_{e} x_{b} /\left(1-x_{r} x_{b}\right)
$$

Similarly, the probability of particles receiving one or more coats $\left(P_{k c}\right)$ is given by

$$
P_{I c}=\left(\begin{array}{l}
1 \\
1
\end{array}\right) x_{e} x_{c}+\left(\begin{array}{l}
2 \\
1
\end{array}\right) x_{e} x_{r} x_{c} x_{b}+\left(\begin{array}{l}
3 \\
1
\end{array}\right) x_{e} x_{r}^{2} x_{c} x_{b}^{2}+\cdots+\left(\begin{array}{l}
n \\
1
\end{array}\right) x_{e} x_{r}^{n-1} x_{c} x_{b}^{n-1}+\cdots
$$


Table 4.2: Selected infinite series definitions.

$$
\begin{aligned}
f\{x\} & =\frac{1}{1-x}=\sum_{n=0}^{\infty} x^{n}=1+x+x^{2}+x^{3}+\cdots \\
f^{\prime}\{x\} & =\frac{1}{(1-x)^{2}}=\sum_{n=1}^{\infty} n x^{n-1}=1+2 x+3 x^{2}+4 x^{3}+\cdots \\
f^{\prime \prime}\{x\} & =\frac{2}{(1-x)^{3}}=\sum_{n=2}^{\infty} n(n-1) x^{n-2}=2+6 x+12 x^{2}+20 x^{3}+\cdots \\
f^{\prime \prime \prime}\{x\} & =\frac{6}{(1-x)^{4}}=\sum_{n=3}^{\infty} n(n-1)(n-2) x^{n-3}=6+24 x+60 x^{2}+\cdots \\
& \vdots \\
f^{k}\{x\} & =\frac{k !}{(1-x)^{k+1}}=\sum_{n=k}^{\infty} \frac{n !}{(n-k) !} x^{n-k}
\end{aligned}
$$

$$
\begin{aligned}
& =\sum_{n=1}^{\infty} n x_{e} x_{r}^{n-1} x_{c} x_{b}^{n-1} \\
& =x_{e} x_{c} /\left(1-x_{r} x_{b}\right)^{2} \\
P_{2 c} & =\left(\begin{array}{l}
2 \\
2
\end{array}\right) x_{e} x_{r} x_{c}^{2}+\left(\begin{array}{l}
3 \\
2
\end{array}\right) x_{e} x_{r}^{2} x_{c}^{2} x_{b}+\left(\begin{array}{l}
4 \\
2
\end{array}\right) x_{e} x_{r}^{3} x_{c}^{2} x_{b}^{2}+\cdots+\left(\begin{array}{l}
n \\
2
\end{array}\right) x_{e} x_{r}^{n-1} x_{c} x_{b}^{n-1}+\cdots \\
& =\sum_{n=2}^{\infty}\left(\begin{array}{l}
n \\
2
\end{array}\right) x_{e} x_{r}^{n-1} x_{c}^{2} x_{b}^{n-2} \\
& =x_{e} x_{c}^{2} x_{r} /\left(1-x_{r} x_{b}\right)^{3} \\
& \vdots \\
P_{k c} & =x_{e} x_{c}^{k} x_{r}^{k-1} /\left(1-x_{r} x_{b}\right)^{k+1}
\end{aligned}
$$

where $x_{c}=1-x_{b}$ denotes the probability of a particle going into the spray zone.

The amount of coat $\left(m_{s l}\right)$ each particle receives per pass through the spray zone can be related to the average coat on the particles $\left(\overline{m_{s}}\right)$, 


$$
\overline{m_{s}}=\sum_{k=0}^{\infty} k \cdot m_{s 1} \cdot P_{k c} / \sum_{k=0}^{\infty} P_{k c}=\sum_{k=0}^{\infty} k \cdot m_{s 1} \cdot P_{k c}
$$

since the denominator in the first equality of Equation (4.10) is equal to one. Substituting $P_{k c}$ from Equation (4.9) into Equation (4.10) and applying infinite series definitions, $\overline{m_{s}}$ reduces to

$$
\overline{m_{s}}=m_{s 1} \cdot x_{c} / x_{c}
$$

Assuming all urea particles have the same mass, the sulfur content distribution can be calculated from Equation (4.9). Furthermore, it follows from Equations (4.3) and (4.11) that

$$
\begin{aligned}
X_{s}\{k\} & =\frac{k \cdot m_{s 1}}{k \cdot m_{s 1}+m_{u}}, \text { for } k=0,1,2, \ldots, \infty \\
& =\frac{k \cdot x_{e} \cdot \overline{X_{s}}}{k \cdot x_{e} \cdot \overline{X_{s}}+\left(1-\bar{X}_{s}\right) x_{c}}, \text { for } k=0,1,2, \ldots, \infty
\end{aligned}
$$

The variables $x_{e}, x_{c}$ and $\bar{X}$ must be specified or known in order to use this model.

The average sulfur content $\left(\overline{X_{s}}\right)$ can be determined experimentally by a crush test of the SCU or it may be calculated from the sulfur and urea flow rates. The probability of particles exiting from the fountain, $x_{e}$, may be determined if the circulation rate $\left(W_{c}\right)$ is known;

$$
x_{c}=W_{p} / W_{c}
$$

The production rate of SCU $\left(W_{p}\right)$ can be calculated from the urea feed rate $\left(W_{u}\right)$,

$$
W_{p}=W_{u} /\left(1-\overline{X_{s}}\right)
$$

assuming negligible loss of sulfur and urea fines.

The probability of the particles entering the spray zone $\left(x_{c}\right)$ is more difficult to determine. It may be estimated by using the average mass of coating material deposited on the particles per pass; however, such an estimate requires information about the deposition rate of the coat onto the bed particles and the density of particles in the spray zone. This information is not readily available. One method of determining this information is to correlate 
$x_{c}$ and $x_{e}$ to the operating variables. The value of $x_{c}$ may be determined by optimizing $x_{c}$ to fit experimental coat distribution data.

This model can also be modified for operations where the urea feed is forced into the spray zone by moving the feed location near the wall of the column. (Tsai (1986) claimed that such a change in feed location improved SCU quality.) The assumptions used in this model are that all urea feed particles pass through the spray zone, and that the volume of the particles does not change significantly as a result of coating. (See Appendix I for the details of the derivation.) The resulting model equations are

$$
P_{k c}=\frac{x_{e} x_{c}^{k-1} x_{r}^{k-1}}{\left(1-x_{b} x_{r}\right)^{k}}, \text { for } k=1,2, \ldots, \infty
$$

and $\quad \overline{X_{s}}\{k\}=\frac{k \cdot x_{s} \overline{X_{s}}}{k \cdot x_{r} \overline{X_{s}}+\left(x_{b} x_{c}+x_{c}\right)\left(1-\overline{X_{s}}\right)}$ for $k=1,2, \ldots, \infty$

The assumption that all particles receive equal amounts of coat as they pass through the spray zone is questionable since the spray concentration decreases with height. Particles which enter the spray zone further away from the nozzle will be exposed to a lower sulfur droplet concentration and spend less time in the spray zone. Such particles receive less coating material than the particles that enter the spray zone closer to the nozzle. In the following section, a model which addresses such a spray zone is developed.

\subsubsection{Model III: Variable Concentration Spray Zone Model}

The purpose of developing Model III is to see the effects of varying the sulfur concentration in the spray zone on sulfur content distribution. The sulfur droplet concentration $\left(C_{c}\right)$ in the spray zone is assumed to vary with the distance from the nozzle, i.e.,

$$
C_{c} \propto\left(z_{c}-z\right)^{a}
$$


where $z_{c}=$ height of the spray zone, $z=$ distance from the nozzle, and $a=$ index. The amount of coat a particle receives is assumed to be directly proportional to the sulfur droplet concentration in the spout, i.e.,

$$
m_{s 1} \propto C_{c}
$$

Therefore, it follows that

$$
m_{s 1}=k_{c}\left(z_{c}-z\right)^{a}
$$

The probability of a particle entering at any location in the spray zone is the same; therefore, the probability density $(\Phi\{z\})$ may be expressed as

$$
\Phi\{z\}=k_{p}
$$

Since $\int_{0}^{z_{c}} \Phi\{z\} d z=x_{c}$, then $k_{p}=x_{c} / z_{c}$. The average amount of coat a particle receives $\left(\overline{m_{s}}\right)$ is given by

$$
\overline{m_{s 1}}=\int_{0}^{z_{c}} m_{s 1} \Phi\{z\} d z / \int_{0}^{z_{c}} \Phi\{z\} d z
$$

which, after solving for $k_{c}$ and substituting into Equation (4.19), gives

$$
m_{s 1}=(a+1) \overline{m_{s 1}}\left(z_{c}-z\right)^{a} / z_{c}^{a}
$$

All other assumptions are the same as for Model II.

Due to the complexity of deriving an analytical expression for Model III, the Monte Carlo method was applied to find the coating distribution. See Section 4.2.3 for more details.

\subsection{Model IV: Rigorous Model}

The ultimate goal of the present study is to model the sulfur coating of urea in a spouted bed by developing sub-models for particle circulation and coating mechanism, and then, based on the histories of single particles, determine the coating distribution. This model should require only the operating and design specifications as inputs and should not depend on other information to determine the coating distribution. 
The approach taken to develop the mathematical model for predicting the concentrations of the sulfur droplets in the gas phase (i.e. spray zone) in the spouted bed is similar to that taken by Meisen and Mathur (1974) for a spouted bed aerosol collector. The bed is divided into three regions as shown in Figure 1.1. Each region is considered separate and distinct from each other although interactions exist along the boundaries. The hydrodynamics and coating in the fountain are expected to be unimportant and, therefore, they are not considered further in this work.

Several key assumptions are required for Model IV. The particles in the fountain are assumed to be perfectly mixed, while those in the spout and annulus are assumed to be in plug flow. In addition, particle segregation due to changing size and density is assumed to be absent. The change in sulfur properties resulting from solidification and allotropic transformations is neglected as far as their effect on bed hydrodynamics, bed temperature and particle circulation is concerned. Consequently the effects such as the influence of bed temperature on the quality of coating (Tsai, 1986; Weiss, 1981) cannot be determined from Model IV.

The Model IV equations and methods used to calculate the bed hydrodynamics, sulfur droplet concentration and coating distribution are outlined in the following sections. The computer program listings for Model IV are given in Appendix III.

\subsubsection{Calculation of Solids Circulation Rate and Bed Hydrodynamics}

The hydrodynamics model used in this work is based on the one-dimensional gas and solids mass and momentum balances for the spout developed by Lefroy and Davidson (1969) and the vector form of the Ergun (1952) equation for gas flow in the annulus. The spout diameter is assumed constant and estimated using Equation (2.4). The pressure distribution at the spout-annulus interface (equal to the radially averaged pressure in the spout) is determined by Equation (2.11) and 


$$
P_{a}\{z\}=\Delta P_{m s}\{H\}-\Delta P_{m s}\{z\}
$$

Equation (4.23) is based on the findings of Grbavcic et al. (1976) that the axial pressure gradient at any elevation above the spout inlet is independent of the height of the bed. By specifying the pressure distribution at the interface, the annulus gas flow problem can be uncoupled from the hydrodynamics in the spout.

The vector Ergun equation, as suggested by Stanek and Szekely (1974) and modified for the annulus by Rovero et al. (1983), is given by

$$
-\nabla P_{a}=\mathbf{U}\left(f_{1}+f_{2}|\mathbf{U}|\right)
$$

where $f_{1}=150 \mu\left(1-\varepsilon_{a}\right)^{2} / d_{p}^{2} \varepsilon_{a}^{3}$

and $f_{2}=1.75 \rho\left(1-\varepsilon_{a}\right) / d_{p} \varepsilon_{a}^{3}$

The equation is solved subject to atmospheric pressure at the top of the annulus, the extended Morgan-Littman distribution at the spout-annulus interface and zero normal pressure gradient at the cylindrical and conical walls of the column. Because the method for solving Equation (4.24) is lengthy and is very similar to the method outlined by Rovero et al., it is given in Appendix I. It should be noted, however, that second order finite difference equations are used to solve for the fluid stream functions (see Appendix I), from which the gas velocity components and pressure can be calculated. Additional assumptions are that $\varepsilon_{a}=\varepsilon_{m f}$ and that $\left|\nu_{a}\right|<<\left|u_{a}\right|$. The solution of the equation governing fluid in the annulus also provides the leakage flow $\left(U_{r}\right)$ of gas from the spout. The latter is required by the gas mass balance in the spout.

The mass and momentum balances of Lefroy and Davidson (1969) for gas and solids motion in the spout are given by

$$
\begin{aligned}
& A_{s} \frac{d\left(\varepsilon_{s} u_{s}\right)}{d z}+\pi D_{s} U_{r}=0 \\
& A_{s} \frac{d\left(\left(1-\varepsilon_{s}\right) v_{s}\right)}{d z}+\pi D_{s} V_{r}=0
\end{aligned}
$$




$$
\begin{aligned}
& \rho A_{s} \frac{d\left(\varepsilon_{s} u_{s}^{2}\right)}{d z}=-\varepsilon_{s} A_{s} \frac{d P_{s}}{d z}-\beta_{p}\left(u_{s}-v_{s}\right)\left|u_{s}-v_{s}\right| A_{s} \\
& \begin{aligned}
\rho_{p} A_{s} \frac{d\left(\left(1-\varepsilon_{s}\right) v_{s}^{2}\right)}{d z}=-\left(1-\varepsilon_{s}\right) A_{s} \frac{d P_{s}}{d z} & +\beta_{p}\left(u_{s}-v_{s}\right)\left|u_{s}-v_{s}\right| A_{s} \\
& -\left(1-\varepsilon_{s}\right)\left(\rho_{p}-\rho\right) A_{s} g
\end{aligned}
\end{aligned}
$$

Equations (4.27) and (4.28) represent the gas and solids mass balances while Equations (4.29) and (4.30) are the gas and solids momentum balances, respectively. The terms on the right hand sides of the momentum equations account for the normal stress, drag between the two phases and, in case of solids, the gravity force. The particle-fluid interaction parameter $\left(\beta_{p}\right)$ is approximated by the Richardson and Zaki (1954) equation as applied by Lefroy and Davidson (1969):

$$
\beta_{p}=0.33\left(1-\varepsilon_{s}\right) \rho / d_{p} \varepsilon_{s}^{178}
$$

The initial conditions for Equations (4.27), (4.29) and (4.30) are:

$$
\varepsilon_{s}=1, u_{s}=u_{0} \text { and } v_{s}=0 \text { at } z=0
$$

This set of equations is solved numerically using UBC ODEPACK (Moore, 1989) running under the UBC MTSG (Runnals, 1987) main frame operating system. Once $\varepsilon_{s} u_{s}$ and $v_{s}$ have been obtained, the particle entrainment flux $\left(V_{r}\right)$ at the spout-annulus interface can be calculated from Equation (4.28).

Finally, the radially averaged particle velocity in the annulus can be determined by equating the solids up-flow in the spout with the solids down-flow in the annulus at any height (z), i.e.,

$$
v_{a}=v_{s} A_{s}\left(1-\varepsilon_{s}\right) / A_{a}\left(1-\varepsilon_{a}\right)
$$

As well as radial uniformity, Equation (4.33) assumes that there is no particle segregation in the annulus and negligible mass change due to coating in the spout. 


\subsubsection{Determination of Coating Mechanism and Concentration Profile}

In determining the coating mechanism and the concentration of sulfur droplets in the spouted bed, it is assumed that the solidification of sulfur droplets, as mentioned earlier, does not occur in the gas phase. Furthermore, it is established from the spray studies (see Section 5.2.3) that the primary mechanism for sulfur droplet deposition on the bed particles is inertial impaction. The latter phenomenon occurs when the gas carrying small sulfur droplets approaches the bed particles; the gas deflects around the particles, while the sulfur droplets, by virtue of their greater inertia, deposit on the bed particles. The validity of the assumption that inertial deposition is the dominant collection mechanism is based on the measured droplet size distribution and information about aerosol collection provided by Clift et al. (1981).

The inertial impaction coating deposition rate $\left(N_{i}\right)$ on the spherical bed particles per unit volume of the bed is calculated from

$$
N_{i}=C_{s} A_{p} \eta|u-v|
$$

where the projected area of particles per unit bed volume $\left(A_{p}\right)$ is

$$
A_{p}=1.5(1-\varepsilon) / d_{p}
$$

The expressions given by Behie et al. (1972), i.e., Equations (2.23) - (2.26), were used to determine the inertial impaction efficiency $(\eta)$.

Two additional assumptions are used to calculate the sulfur spray concentration. First, the sulfur spray is presumed not to penetrate into the annulus (i.e., coating only occurs in the spout), since the spray occupies a very small volume of the spout (see Figure 4.5). Second, coating does not occur until the spray reaches the spout-annulus interface (at which point the spray is assumed to be fully developed). The second assumption was based on visual observations of the bed particles in a half column spouted bed and of the sulfur spray in the spray box. The concentration of the bed particles is low in the lower section 


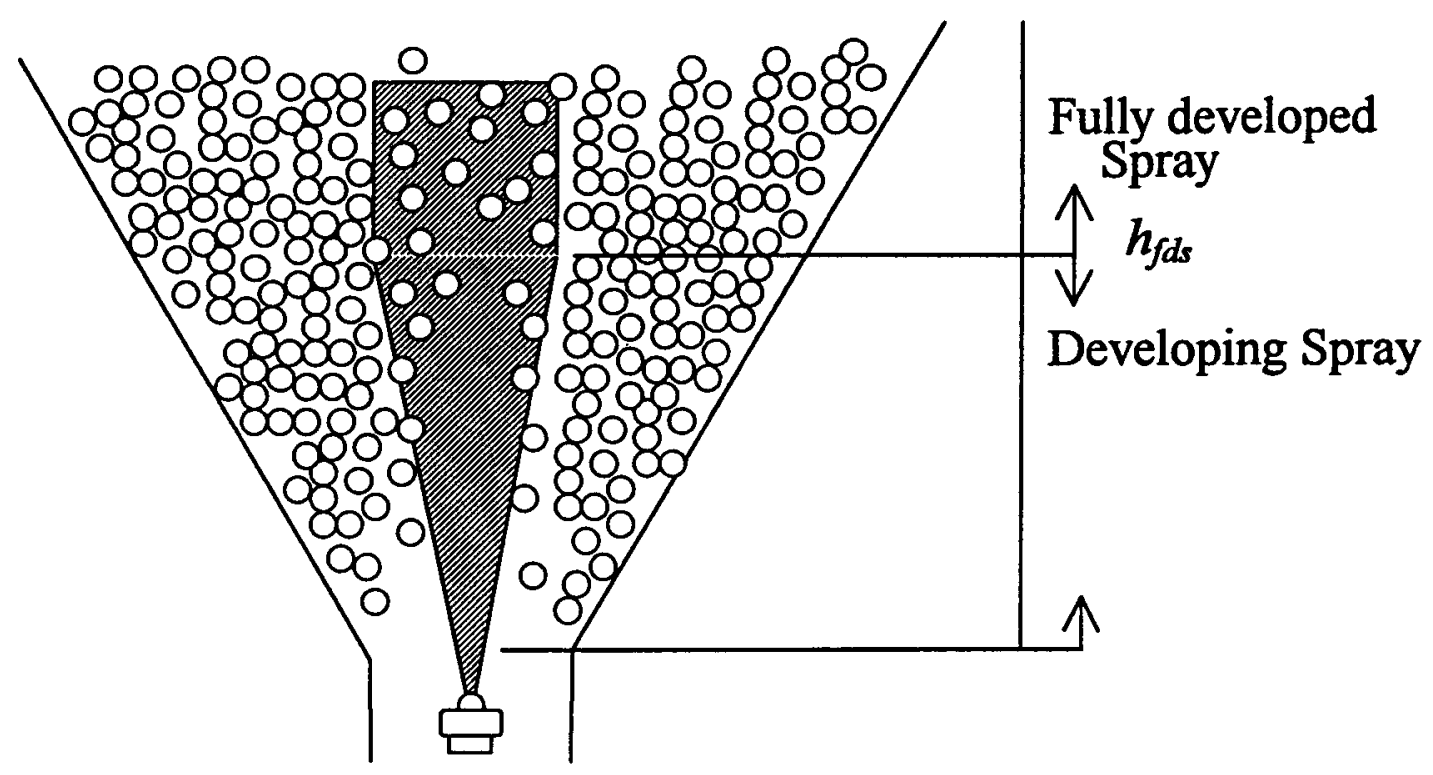

Figure 4.5: Sectional view of the lower spout.

of the spout and the particles are located primarily near the wall of the spout. The spray only occupies a small area just above the nozzle.

With these assumptions, a mass balance for coating material in the spout gives (see Figure 4.6):

$$
\begin{aligned}
& \frac{\mathrm{d}\left(C_{s} u_{s} \varepsilon_{s} A_{s}\right)}{\mathrm{d} z}=0, \text { for } z<h_{f d s} \\
& \frac{\mathrm{d}\left(C_{s} u_{s} \varepsilon_{s} A_{s}\right)}{\mathrm{d} z}+N_{i} A_{s}=0, \text { for } z \geq h_{f d s}
\end{aligned}
$$

The initial condition is

$$
C_{s}=C_{s 0} \text { at } z=0
$$

Note that Equation (4.38) assumes that the sulfur droplet concentration below $z=h_{f d s}$ can be smeared out radially, in order that a one-dimensional approximation may be applied. Equations (4.36) and (4.37) are solved simultaneously with Equations (4.27) to (4.30) using UBC ODEPACK. The spray angle of $20^{\circ}$ (provided by the manufacturer of the spray nozzle for spraying water into still air) is used in the calculations to determine $h_{f d s}$. 


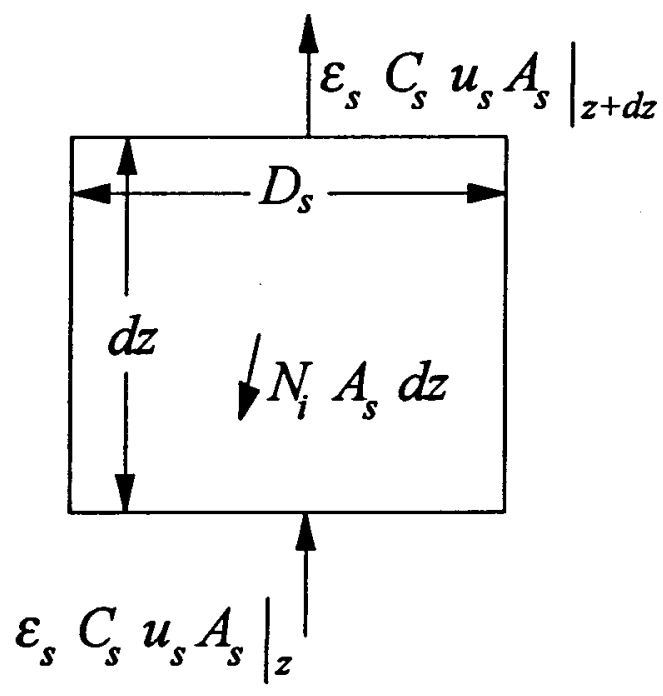

Figure 4.6: Mass balance on coating material in the spout.

\subsubsection{Calculation of Coating Distribution Using the Monte Carlo Method}

\subsubsection{Limitation of Analytical Model}

The equations governing Models I and II can be solved analytically. Such models are called deterministic models. However, Model III and Model IV are not amenable to analytical solutions. For example Models II - IV may be represented by

$$
\operatorname{Pr}\left\{m_{s} \leq m_{s}^{*}\right\}=\sum_{i=1}^{\infty} \operatorname{Pr}\{N=i\} \operatorname{Pr}\left\{\sum_{j=1}^{i} m_{s 1} \leq m_{s}^{*} \mid N=i\right\}
$$

where $\operatorname{Pr}\left\{m_{s} \leq m_{s}^{*}\right\}$ is the probability that no more than $m_{s}^{*}$ is deposited on a particle during its residence in the bed. $\operatorname{Pr}\{N=i\}$ denotes the probability that a particle stays in the bed for $i$ cycles, and $\operatorname{Pr}\left\{\sum_{j=1}^{i} m_{s 1} \leq m_{s}^{*} \mid N=i\right\}$ is the conditional probability that the total coat a particle receives is less than $m_{s}^{*}$ given that the particle goes through $i$ cycles before exiting the bed. From the analysis of the exit probabilities, it can be shown that 


$$
\operatorname{Pr}\{N=i\}=x_{e} x_{b}^{i-1}
$$

Mann (1983) showed that the remainder of the term in Equation (4.39) can be reduced to

$$
\operatorname{Pr}\left\{\sum_{j=1}^{i} m_{s 1} \leq m_{s}^{*} \mid N=i\right\}=\operatorname{Pr}^{*}\left\{m_{s 1} \leq m_{s}^{*} \mid N=i\right\}
$$

where $\operatorname{Pr}^{*_{i}}\left\{m_{s 1} \leq m_{s}^{*} \mid N=i\right\}$ denotes the $i$ th order convolution of $\operatorname{Pr}\left\{m_{s 1} \leq m_{s}^{*} \mid N=i\right\}$. Simple analytical expressions can be obtained if an exact solution of the convolution in Equation (4.41) exists and if an infinite series representation exists for Equation (4.39). If both conditions are satisfied, Equation (4.39) can be reduced to a simple analytical expression. The equation can usually be solved numerically if the conditions are not satisfied. However, the derivations of analytical expressions or numerical solutions can be difficult for complicated models such as Models III and IV, and are susceptible to errors from dealing with large arrays of coating probabilities or from numerical procedures. Moreover, both processes must be repeated if model specifications change (e.g., change in feed location for Model II). The errors associated with these methods can be avoided, and the repeat derivations are unnecessary if the Monte Carlo method is used.

\subsubsection{Monte Carlo Procedure for Model III}

The path of a particle and the sulfur deposited on the particle are simulated from given values of $x_{e}, x_{c}$ and $a$, and random parameters $N_{R I}$ and $N_{R 2}$. As each particle enters the bed, its path through the spout is determined by $N_{R I}$, from which $m_{s I}$ can be calculated

$$
\begin{aligned}
& m_{s 1}=0, \text { for } N_{\mathrm{R} 1}>x_{c} \\
& m_{s 1}=(a+1) \overline{m_{s 1}}\left(z_{c}-z\right)^{a} / z_{c}^{a}, \text { for } N_{\mathrm{R} 1} \leq x_{c}
\end{aligned}
$$

where $z=N_{R 1} \cdot H, 0<N_{R 1}<1$ and $z_{c}$ is the distance indicating the upper limit of the spray zone. Once the particle leaves the spout and enters the fountain, its path from the fountain is determined by $N_{R 2}$, i.e., 


$$
\begin{aligned}
& \text { if } N_{R 2}<x_{e}, \text { the particle leaves the bed } \\
& \text { if } N_{R 2} \geq x_{e} \text {, the particle stays in the bed }
\end{aligned}
$$

where $0<N_{R 2}<1$. If the particle stays in the bed, the procedure is repeated. If the particle leaves the bed, another particle is introduced until a sufficient number of particles is simulated to determine the coating distribution. The value of $\overline{m_{s 1}}$ is determined from the following definition of the mean sulfur weight in the product $\left(\bar{m}_{s}\right)$ :

$$
\overline{m_{s}} \approx \frac{\sum \text { coating on the particles in units of } \overline{m_{s 1}}}{\text { number of particles sampled }}
$$

and the sulfur content is found by applying Equation (4.3). The random numbers $N_{R I}$ and $N_{R 2}$ are generated using the UBC RANDOM (Nicol, 1986) package available in UBC MTSG (Runnals, 1987). A pseudo-random number generator was used, i.e. the same 'seed' value produces the same sequence of random number.

\subsubsection{Monte Carlo Procedure for Model IV}

The Monte Carlo method (see Section 2.5) was used to determine the distribution of sulfur on the particles leaving the spouted bed. The sulfur deposit on a particle is determined by following the path of the particle in the bed and calculating the amount of coating material the particle receives until it leaves the bed. The procedure is then repeated for another feed particle until a sufficient number of particles has been considered to give a good representation of the coating distribution. The detailed procedure for determining the coat on an individual particle during its residence in a spouted bed is given in the following subsections:

\subsection{Contimuous Operation}

1. When a feed particle enters the unit or a cycling particle returns via the fountain to the top of the bed, its radial position at the top of the annulus is randomly assigned. The feed urea particle, as it enters the bed, is assumed to land within $\left(\Delta D_{f}=0.03 \mathrm{~m}\right.$ of the column 
wall at the surface of the annulus. This assumption is based on the observations that majority of particles fall within $0.03 \mathrm{~m}$ of the column. The radial position of the feed particle on the annulus surface is assigned using a random number generated by the UBC RANDOM (Nicol, 1986) package available in the MTSG (Runnals, 1987) main frame operating system at UBC. The generated number $\left(N_{R}\right)$ falls between 0 and 1 , and it is used to assign the radial position $\left(D_{r}\right)$ of the particle according to

$$
N_{R}=\frac{D_{r}^{2}-D_{f}^{2}}{D^{2}-D_{f}^{2}}
$$

where $D_{f}=D-\Delta D_{f}$

$\Delta D_{f}$ denotes twice the width (adjacent to the bed wall) of the bed surface area occupied by the feed and $D$ denotes the column diameter. Similarly, the radial position of the returning particle (via the fountain) on the annulus is calculated from

$$
\begin{aligned}
& N_{R}=\frac{D_{r}^{2}-D_{s}^{2}}{\left(D_{f}^{2}-D_{s}^{2}\right)+\left(1-x_{f}\right)\left(D^{2}-D_{f}^{2}\right)}, \text { for } D_{r} \geq D_{f} \\
& N_{R}=\frac{\left(1-x_{f}\right)\left(D_{r}^{2}-D_{f}^{2}\right)+\left(D_{f}^{2}-D_{s}^{2}\right)}{\left(D_{f}^{2}-D_{s}^{2}\right)+\left(1-x_{f}\right)\left(D^{2}-D_{f}^{2}\right)}, \text { for } D_{r}<D_{f}
\end{aligned}
$$

where $x_{f}=\frac{W_{u} / \rho_{u}}{v_{a}\left(D^{2}-D_{f}^{2}\right)\left(1-\varepsilon_{a}\right) \pi /\left.4\right|_{H}}$

The denominator in Equations (4.49) and (4.50) represents the total area occupied by the recycling particles, and $x_{f}$ represents the fraction of area the feed particles occupy in the area defined by $\pi\left(D^{2}-D_{f}^{2}\right) / 4$.

2. The particle is followed around the bed, i.e. entry into the annulus, transport in the annulus, entry into the spout, transport in the spout, entry into the fountain, transport in the fountain, entry into the discharge tube or re-entry into the annulus. As the particle spends 
significantly more time in the annulus than the spout or fountain, the total cycle time $\left(t_{c}\right)$ is estimated using the residence time of the particle in the annulus:

$$
t_{c}=\int_{H}^{z_{s}} d z / v_{a}\{z\}
$$

$z_{s}$ can be determined by equating the particle flow rate at the surface of the annulus between $D$ and $D_{r}$ to that in the spout at $z_{s}$, i.e.,

$$
\left.v_{a}\left(1-\varepsilon_{a}\right)\left(D_{c}^{2}-D_{r}^{2}\right)\right|_{H}=\left.v_{s}\left(1-\varepsilon_{s}\right) D_{s}^{2}\right|_{z_{s}}
$$

where $\left.v_{a}\right|_{H}=\left.v_{s} \frac{1-\varepsilon_{s}}{1-\varepsilon_{a}} \frac{D_{s}^{2}}{D^{2}-D_{s}^{2}}\right|_{H}$

The total residence time $\left(t_{r}\right)$ in the bed can be found from the sum of the cycle times

$$
t_{r}=\sum_{i=1}^{N} t_{c}
$$

where $N$ denotes the total number of cycles a particular particle makes before leaving the bed. The coating amount per cycle $\left(m_{s 1}\right)$ can be calculated from

$$
\begin{aligned}
& m_{s 1}=\int_{z_{s}}^{H} A_{p}^{*} C_{s}\left|u_{s}-v_{s}\right| \eta / v_{s} \mathrm{~d} z, \text { for } z_{s}>h_{f d s} \\
& m_{s 1}=\int_{h_{f d s}}^{H} A_{p}^{*} C_{s}\left|u_{s}-v_{s}\right| \eta / v_{s} \mathrm{~d} z, \text { for } z_{s} \leq h_{f d s}
\end{aligned}
$$

where $A_{p}{ }^{*}$ is the projected area of a single urea particle and $h_{f d s}$ is the height at which the spray becomes fully developed and, hence, coating occurs beyond this point (see Figure 4.6). The spray angle $(\phi)$ was assumed to be $20^{\circ}$ and $h_{f d s}$ is calculated from

$$
h_{f d s}=D_{s} \tan (\phi / 2) / 2
$$

Since coating is assumed not to occur in the annulus and fountain, $m_{s 1}$ in Equations (4.56) and (4.57) represent the total amount of sulfur deposited on the particle in one cycle.

3. The position of the particle is reassigned on the annulus surface. If the particle lands in the exit stream, the simulation ends for this particular particle and begins for another. Otherwise, the next cycle begins as before. 
The sulfur content $\left(X_{s p, i}\right)$ of the ith particle leaving the bed is given by

$$
X_{s p, i}=\Sigma_{j=1}^{N} m_{s 1} /\left(\Sigma_{j=1}^{N} m_{s 1}+m_{k}\right)
$$

where $m_{u}$ is the mass of urea in a single bed particle. Because the average sulfur content of simulated particles $\left(\bar{X}_{s p}\right)$ does not always agree with the sulfur content calculated from the sulfur and urea feed rates $\left(\bar{X}_{s}\right)$, especially when a small number of particles are simulated, $X_{s p, i}$ is normalized as follows:

$$
X_{s p, i}=X_{s p, i} \bar{X}_{s p} / \bar{X}_{s}
$$

In total, 1000 particles were typically simulated to determine the coating distribution for each set of operating condition. The values of $\bar{X}_{s}$ and $\bar{X}_{s p}$ typically differed by $2 \%$ before normalizing according to Equation (4.60). The effect of the sample size is discussed in Chapter 5.

\subsection{Batch Operation}

The simulation procedure for the batch coating operation is the same as that for the continuous operation except that the start and the termination of the particle simulation are different. The simulation of batch coating terminates when the particle's residence time is equal to the total coating time of the batch product. The cycle and residence times are calculated from Equations (4.52) and (4.55). The detailed derivation of the batch model is given in Appendix I. 


\section{Chapter 5. \\ Results and Discussions}

The results of the studies conducted on the bed hydrodynamics and sulfur spray are discussed in the first two sections of this chapter. The third section deals with the coating experiments including the coating distribution and product quality. The commercial implications of the present work are then discussed.

\subsection{Bed Hydrodynamics}

The minimum spouting velocity, pressure in the annulus, and fluid velocity in the spout were measured in the absence of sulfur injection. The validity of expressions presented in Chapter 2 were compared with the experimental data obtained from the present coating unit. Modifications were made in the expressions to improve their predictive ability where necessary and possible. The modifications resulted from the presence of the pneumatic atomizing nozzle in the spouting air inlet, the presence of atomizing air, and operating with shallow conical beds.

The ranges of variables examined in this study are summarized in Table 5.1. A total of 327 runs were performed to determine the minimum spouting velocity and the spout velocity and annulus pressure profiles were measured in 32 runs. The operating conditions of the experiments performed to determine the pressure profiles and spout velocities are given in Table 5.2. Pressure and velocity data are given in Tables 5.3 and 5.4, respectively. The minimum spouting velocity data are provided in Appendix II. 
Table 5.1: Operating ranges applicable to this work.

\begin{tabular}{|cc|cc|}
\hline Parameter & Operating Range & Parameter & Operating Range \\
\hline$H$ & $0.11-0.63 \mathrm{~m}$ & $Q_{a}$ & $0-0.87 \mathrm{~L} / \mathrm{s}$ \\
$D$ & 0.24 and $0.45 \mathrm{~m}$ & $T$ & $18-70^{\circ} \mathrm{C}$ \\
$d_{i}$ & $21-35 \mathrm{~mm}$ & $\rho$ & $1-1.2 \mathrm{~kg} / \mathrm{m}^{3}$ \\
$Q_{s}$ & $0-37 \mathrm{~L} / \mathrm{s}$ & & \\
\hline
\end{tabular}

\subsubsection{Minimum Spouting Velocity, $\boldsymbol{U}_{m s}$}

Besides providing the lower limit of operation, the minimum spouting velocity $\left(U_{m s}\right)$ information may be used to study the effects on bed hydrodynamics of atomizing air, presence of the nozzle, and operating with shallow conical beds.

Figure 5.1 provides comparison between the measured $U_{m s}$ and the values calculated by the standard equations due to Mathur and Gishler (1955) and Wu et al. (1987) (given by Equations (2.2) and (2.3), respectively). The values of the constants in Equation (2.3) are repeated in Table 5.5.

As shown by Figure 5.1, poor agreement was obtained between the experimental measurements and the aforementioned standard equations. The discrepancy was thought to result from determining the experimental $U_{m s}$ values based on the total air flow rate (i.e., the sum of $Q_{a}$ and $Q_{s}$ ) without due regard to the momentum of the main and atomizing air streams. The atomizing air, which enters at high velocity (up to $131 \mathrm{~m} / \mathrm{s}$ ) in the centre of the spout, should enhance the effectiveness of the main spouting air stream (typical velocities: 22 to $78 \mathrm{~m} / \mathrm{s}$ ) and therefore reduce the minimum spouting velocity. This is confirmed by the radial velocity profile measured at the bottom of the cone (just above the shutter) in the absence of bed particles (see Figure 5.2). 
Table 5.2: Operating conditions under which the air velocities in the spout and the pressure profiles in the annulus were determined.

\begin{tabular}{|c|c|c|c|c|c|c|c|c|}
\hline $\begin{array}{c}\text { Run } \\
\#\end{array}$ & Particle Type & $\begin{array}{c}Q_{s} \\
\mathrm{~L} / \mathrm{s}\end{array}$ & $\begin{array}{r}Q_{a} \\
\mathrm{~L} / \mathrm{s} \\
\end{array}$ & $\begin{array}{l}H \\
\mathrm{~m} \\
\end{array}$ & $\begin{array}{l}D \\
\mathrm{~m} \\
\end{array}$ & $\begin{array}{l}d_{i} \\
\mathrm{~m} \\
\end{array}$ & $\begin{array}{c}T \\
{ }^{\circ} \mathrm{C} \\
\end{array}$ & $\begin{array}{c}\rho \\
\mathrm{kg} / \mathrm{m}^{3}\end{array}$ \\
\hline H1 & Polyformaldehyde & 33.77 & 0.53 & 0.29 & 0.24 & 0.0308 & 60 & 1.06 \\
\hline $\mathrm{H} 2$ & Polyformaldehyde & 32.96 & 0.53 & 0.29 & 0.24 & 0.0308 & 66 & 1.04 \\
\hline $\mathrm{H} 3$ & Polyformaldehyde & 28.50 & 0.53 & 0.29 & 0.24 & 0.0308 & 65 & 1.05 \\
\hline $\mathrm{H} 4$ & Polyformal & 29.65 & 0.53 & 0.29 & 0.24 & 0.0308 & 61 & 1.06 \\
\hline $\mathrm{H} 5$ & Polyt & 31.97 & 0.87 & 0.29 & 0.24 & 0.0308 & 67 & 1.04 \\
\hline $\mathrm{H} 6$ & Polyt & 31.89 & 0.70 & 0.29 & 0.24 & 0.0308 & 68 & 1.04 \\
\hline $\mathrm{H} 7$ & Poly & 31.53 & 0.53 & 0.29 & 0.24 & 0.0308 & 66 & 1.04 \\
\hline $\mathrm{H} 8$ & Poly & 31.19 & 0.00 & 0.29 & 0.24 & 0.0308 & 68 & 1.04 \\
\hline $\mathrm{H} 9$ & Pol & 33.15 & 0.53 & 0.29 & 0.24 & 0.0351 & 68 & 1.04 \\
\hline $\mathrm{H} 10$ & $\mathrm{Pol}$ & 31.82 & 0.53 & 0.29 & 0.24 & 0.0282 & 70 & 1.03 \\
\hline H11 & & 30.47 & 0.53 & 0.29 & 0.24 & 0.0247 & 71 & 1.03 \\
\hline $\mathrm{H} 12$ & Pol & 27.78 & 0.53 & 0.29 & 0.24 & 0.0212 & 66 & 1.04 \\
\hline H13 & Pol & 28.65 & 0.53 & 0.29 & 0.24 & 0.0308 & 21 & 1.20 \\
\hline H14 & de & 33.77 & 0.53 & 0.34 & 0.24 & 0.0308 & 66 & 1.06 \\
\hline H15 & de & 33.97 & 0.53 & 0.37 & 0.24 & 0.0308 & 62 & 1.05 \\
\hline H16 & Poly & 36.02 & 0.53 & 0.37 & 0.24 & 0.0308 & 66 & 1.04 \\
\hline H17 & Poly & 34.17 & 0.53 & 0.53 & 0.24 & 0.0308 & 64 & 1.05 \\
\hline H18 & Poly & 28.50 & 0.53 & 0.34 & 0.24 & 0.0308 & 65 & 1.05 \\
\hline H19 & Poly & 31.81 & 0.53 & 0.36 & 0.24 & 0.0308 & 69 & 1.03 \\
\hline H20 & Poly & 27.54 & 0.53 & 0.29 & 0.24 & 0.0308 & 62 & 1.05 \\
\hline H21 & Poly & 29.48 & 0.53 & 0.30 & 0.45 & 0.0308 & 60 & 1.06 \\
\hline $\mathrm{H} 22$ & Poly & 31.17 & 0.53 & 0.25 & 0.45 & 0.0308 & 62 & 1.05 \\
\hline $\mathrm{H} 23$ & Pol & 35.39 & 0.53 & 0.31 & 0.45 & 0.0308 & 62 & 1.05 \\
\hline $\mathrm{H} 24$ & Pol & 32.05 & 0.00 & 0.29 & 0.45 & 0.0308 & 62 & 1.05 \\
\hline $\mathrm{H} 25$ & Polyfor & 32.91 & 0.87 & 0.29 & 0.45 & 0.0308 & 62 & 1.05 \\
\hline $\mathrm{H} 26$ & Polyformalc & 31.17 & 0.53 & 0.29 & 0.45 & 0.0308 & 62 & 1.05 \\
\hline $\mathrm{H} 27$ & Polyformaldehyde & 33.97 & 0.53 & 0.29 & 0.45 & 0.0308 & 62 & 1.05 \\
\hline $\mathrm{H} 28$ & Polyformaldehyde & 32.57 & 0.53 & 0.29 & 0.45 & 0.0308 & 62 & 1.05 \\
\hline H29 & Polyformaldehyde & 32.57 & 0.53 & 0.29 & 0.45 & 0.0282 & 62 & 1.05 \\
\hline $\mathrm{H} 30$ & Polyformaldehyde & 30.46 & 0.53 & 0.29 & 0.45 & 0.0247 & 62 & 1.05 \\
\hline H31 & Polyformaldehyde & 35.39 & 0.53 & 0.29 & 0.45 & 0.0351 & 62 & 1.05 \\
\hline 432 & Polyformaldehyde & 32.08 & 0.53 & 0.29 & 0.45 & 0.0308 & 24 & 1.19 \\
\hline
\end{tabular}


Table 5.3: Axial pressure profile near the spout-annulus interface. ( $R$ - Run number as shown in Table 5.2; $z$ - height from the base of the bed, $\mathrm{mm}$; pressures are expressed in $\mathrm{mm}$ water).

a) Small Bed $(D=0.24 \mathrm{~m})$

\begin{tabular}{|c|c|c|c|c|c|c|c|c|c|c|c|c|c|c|c|c|c|c|c|c|c|c|}
\hline Runlz & 15 & 35 & 55 & 75 & 95 & 115 & 135 & 155 & 175 & 195 & 215 & 235 & 255 & 275 & 295 & 315 & 335 & 355 & 385 & 435 & 485 & 535 \\
\hline $\mathrm{Hl}$ & 96 & 92 & 86 & 82 & 78 & 68 & 56 & 48 & 40 & 29 & 22 & 14 & 5 & 0 & - & - & - & - & - & - & - & - \\
\hline $\mathrm{H} 2$ & 86 & 88 & 83 & 78 & 76 & 66 & 57 & 46 & 36 & 32 & 22 & 16 & 8 & 4 & - & - & - & - & - & - & - & - \\
\hline $\mathrm{H3}$ & - & 98 & 92 & 87 & 79 & 73 & 58 & 48 & 39 & 32 & 26 & 18 & 9 & 6 & - & - & - & - & - & - & - & - \\
\hline $\mathrm{H} 4$ & 97 & 98 & 91 & 84 & 78 & 70 & 61 & 50 & 42 & 31 & 20 & 12 & 6 & 2 & - & - & - & - & - & - & - & - \\
\hline H5 & 94 & 96 & 92 & 85 & 80 & 70 & 62 & 49 & 40 & 32 & 25 & 17 & 8 & 2 & - & - & - & - & - & - & $\cdot$ & - \\
\hline H6 & 91 & 92 & 90 & 81 & 76 & 65 & 56 & 46 & 36 & 30 & 21 & 15 & 7 & 0 & - & - & - & - & - & - & - & $\bullet$ \\
\hline $\mathrm{H} 7$ & 89 & 93 & 87 & 82 & 75 & 68 & 60 & 49 & 38 & 28 & 25 & 17 & 6 & 1 & - & - & - & - & - & - & - & - \\
\hline H8 & 97 & 100 & 94 & 89 & 82 & 70 & 65 & 56 & 47 & 39 & 28 & 22 & 13 & 6 & - & - & - & - & - & - & - & - \\
\hline $\mathrm{H} 9$ & 91 & 105 & 94 & 88 & 83 & 73 & 62 & 51 & 41 & 31 & 23 & 14 & 7 & 0 & - & - & - & - & - & - & - & - \\
\hline $\mathrm{HlO}$ & - & 86 & 81 & 79 & 75 & 68 & 61 & 51 & 43 & 36 & 28 & 19 & 10 & 4 & - & - & - & - & - & $\cdot$ & - & - \\
\hline H11 & - & 76 & 76 & 71 & 67 & 64 & 60 & 50 & 40 & 32 & 28 & 21 & 10 & 5 & - & - & - & - & - & - & - & - \\
\hline $\mathrm{H} 12$ & - & 67 & 75 & 71 & 72 & 63 & 59 & 51 & 47 & 40 & 32 & 22 & 8 & 0 & - & - & - & $\bullet$ & - & - & - & - \\
\hline $\mathrm{H} 13$ & - & 82 & 80 & 75 & 68 & 63 & 59 & 49 & 39 & 29 & 19 & 10 & 2 & 0 & - & - & - & - & - & - & - & - \\
\hline $\mathrm{H} 14$ & 102 & 114 & 111 & 108 & 100 & 92 & 86 & 72 & 62 & 54 & 44 & 34 & 28 & 20 & 12 & 1 & 2 & - & - & - & - & - \\
\hline $\mathrm{H} 15$ & - & - & - & - & 107 & 103 & 90 & 85 & 81 & 67 & 62 & 52 & - & 35 & - & 21 & 1 & - & - & - & - & - \\
\hline H16 & 63 & 105 & 104 & 99 & 96 & 83 & 73 & 70 & - & 65 & - & 55 & - & 38 & - & 22 & - & 1 & - & - & - & - \\
\hline $\mathrm{H} 17$ & 154 & 148 & 149 & 140 & 134 & 128 & 128 & 127 & - & 119 & - & 99 & - & - & 86 & - & 78 & - & 52 & 36 & 21 & 1 \\
\hline $\mathrm{H} 18$ & 67 & 62 & 59 & - & 55 & 49 & 47 & - & 37 & - & 27 & - & 20 & - & 7 & - & 0 & - & - & - & - & - \\
\hline H19 & 99 & 95 & 87 & 83 & 82 & 74 & 70 & 64 & - & 49 & - & 35 & - & 25 & - & 19 & - & 2 & - & - & - & - \\
\hline $\mathrm{H} 20$ & 76 & 73 & 66 & 60 & 59 & 52 & 46 & 35 & 26 & 25 & - & 12 & - & 2 & - & - & - & - & - & - & - & - \\
\hline
\end{tabular}

b) Large $\operatorname{Bed}(D=0.45 \mathrm{~m})$

\begin{tabular}{|c|c|c|c|c|c|c|c|c|c|c|c|c|c|c|c|c|}
\hline Runlz & 20 & 40 & 60 & 80 & 100 & 120 & 140 & 160 & 180 & 200 & 220 & 240 & 260 & 270 & 280 & 300 \\
\hline $\mathrm{H} 2 \mathrm{I}$ & 60 & 60 & 56 & 53 & 48 & 44 & - & 34 & - & 23 & - & 11 & - & - & 0 & - \\
\hline $\mathrm{H} 22$ & 74 & 72 & 67 & 62 & 55 & 49 & - & 34 & - & 18 & - & 1 & - & - & - & - \\
\hline $\mathrm{H} 23$ & 91 & 96 & 86 & 80 & 73 & - & 63 & - & 49 & - & 33 & - & 25 & - & - & 0 \\
\hline $\mathrm{H} 24$ & 80 & 80 & 76 & 70 & 64 & - & 51 & - & 35 & - & 17 & - & - & 0 & - & - \\
\hline $\mathrm{H} 25$ & 80 & 77 & 74 & 69 & 62 & - & 50 & - & 34 & - & 19 & - & - & 0 & - & - \\
\hline $\mathrm{H} 26$ & 81 & 81 & 76 & 70 & 64 & - & 51 & - & 35 & - & 18 & - & - & 0 & - & - \\
\hline $\mathrm{H} 27$ & 80 & 80 & 76 & 72 & 64 & - & 51 & - & 35 & - & 18 & - & - & 0 & - & - \\
\hline $\mathrm{H} 28$ & 81 & 85 & 76 & 74 & 63 & - & 53 & - & 36 & - & 22 & - & - & 2 & - & - \\
\hline H29 & 76 & 73 & 70 & 65 & 63 & - & 49 & - & 36 & - & 19 & - & - & 0 & - & - \\
\hline $\mathrm{H} 30$ & 49 & 60 & 60 & 58 & 56 & - & 47 & - & 34 & - & 18 & - & - & 0 & - & - \\
\hline H31 & 97 & 92 & 84 & 80 & 74 & - & 56 & - & 40 & - & 22 & - & - & 1 & - & - \\
\hline H32 & 81 & 81 & 76 & 69 & 59 & - & 48 & - & 32 & - & 12 & - & - & 0 & - & - \\
\hline
\end{tabular}


Table 5.4: Air velocity profile in the spout. ( $R$ - Run number as shown in Table 5.2; $z$-height from the base of the bed, mm; velocities are expressed in $\mathrm{m} / \mathrm{s}$ ).

a) Small Bed $(D=0.24 \mathrm{~m})$

\begin{tabular}{|c|c|c|c|c|c|c|c|c|c|c|c|c|c|c|c|c|c|}
\hline Runlz & 105 & 125 & 145 & 165 & 185 & 205 & 225 & 245 & 265 & 285 & 305 & 325 & 335 & 355 & 405 & 455 & 505 \\
\hline $\mathrm{HI}$ & 37.6 & 30.5 & 27.5 & 24.6 & 21.9 & 19.4 & 18.1 & 15.9 & - & - & - & - & - & - & - & - & - \\
\hline $\mathrm{H} 2$ & 37.6 & 33.2 & 26.8 & 23.2 & 21.2 & 19.0 & 17.5 & 15.2 & - & - & - & - & - & - & - & - & - \\
\hline $\mathrm{H} 3$ & 34.7 & 28.2 & 22.6 & 20.2 & 17.5 & 16.8 & 15.2 & 13.9 & - & - & - & - & - & - & - & - & - \\
\hline $\mathrm{H} 4$ & 35.6 & 29.3 & 24.1 & 20.7 & 18.8 & 17.4 & 15.9 & 14.2 & - & - & - & - & - & - & - & - & - \\
\hline H5 & 39.9 & 32.5 & 26.4 & 22.7 & 21.2 & 19.0 & 17.2 & 15.6 & - & - & - & - & - & - & - & - & - \\
\hline H6 & 39.0 & 30.9 & 25.9 & 23.3 & 20.3 & 18.7 & 16.9 & 14.8 & - & - & - & - & - & - & - & - & - \\
\hline $\mathrm{H} 7$ & 37.9 & 30.0 & 25.3 & 22.7 & 19.6 & 17.9 & 16.8 & 15.2 & - & - & - & - & - & - & - & - & - \\
\hline H8 & 38.0 & 30.1 & 25.9 & 22.1 & 19.0 & 18.0 & 16.5 & 14.8 & - & - & - & - & - & - & - & - & - \\
\hline H9 & 33.3 & 28.3 & 24.4 & 20.9 & 18.7 & 16.9 & 15.7 & 14.4 & - & $\because$ & - & - & - & - & - & - & - \\
\hline $\mathrm{H} 10$ & 35.7 & 30.1 & 25.5 & 22.8 & 19.7 & 18.0 & 15.3 & 13.0 & - & - & - & - & - & - & - & - & - \\
\hline H11 & 31.5 & 27.0 & 23.4 & 20.4 & 18.4 & 16.9 & 14.9 & 13.0 & - & - & - & - & - & - & - & - & - \\
\hline $\mathrm{H} 13$ & - & 13.8 & $17.0^{\circ}$ & 19.5 & 16.3 & 14.9 & 13.8 & 12.5 & 8.8 & - & - & - & - & - & - & - & - \\
\hline $\mathrm{H} 14$ & 40.8 & 32.5 & 27.5 & 24.1 & 20.7 & 18.8 & 15.9 & 15.9 & 15.1 & 14.2 & 12.3 & 7.1 & - & - & - & - & - \\
\hline $\mathrm{H} 16$ & - & 25.3 & 24.3 & 17.5 & 16.8 & 14.3 & 14.3 & 14.3 & 14.3 & 13.4 & 12.4 & 11.9 & 11.9 & - & - & - & - \\
\hline $\mathrm{H} 17$ & - & - & 34.8 & 28.8 & 24.0 & - & 18.2 & - & 14.3 & - & 12.9 & - & - & 10.1 & 10.1 & 10.1 & 8.0 \\
\hline $\mathrm{H} 18$ & 38.4 & 31.8 & 26.5 & 21.2 & 17.9 & - & 14.3 & - & 12.9 & - & 8.8 & - & - & - & - & - & - \\
\hline $\mathrm{H} 19$ & 30.3 & 23.9 & 20.4 & 17.6 & 13.0 & 11.9 & - & 10.8 & - & 10.8 & - & 10.8 & - & - & - & - & - \\
\hline $\mathrm{H} 20$ & 25.9 & 21.1 & 17.1 & 14.2 & 11.3 & 10.1 & 9.4 & 8.0 & 5.0 & - & - & - & - & - & - & - & - \\
\hline
\end{tabular}

b) Large Bed $(D=0.45 \mathrm{~m})$

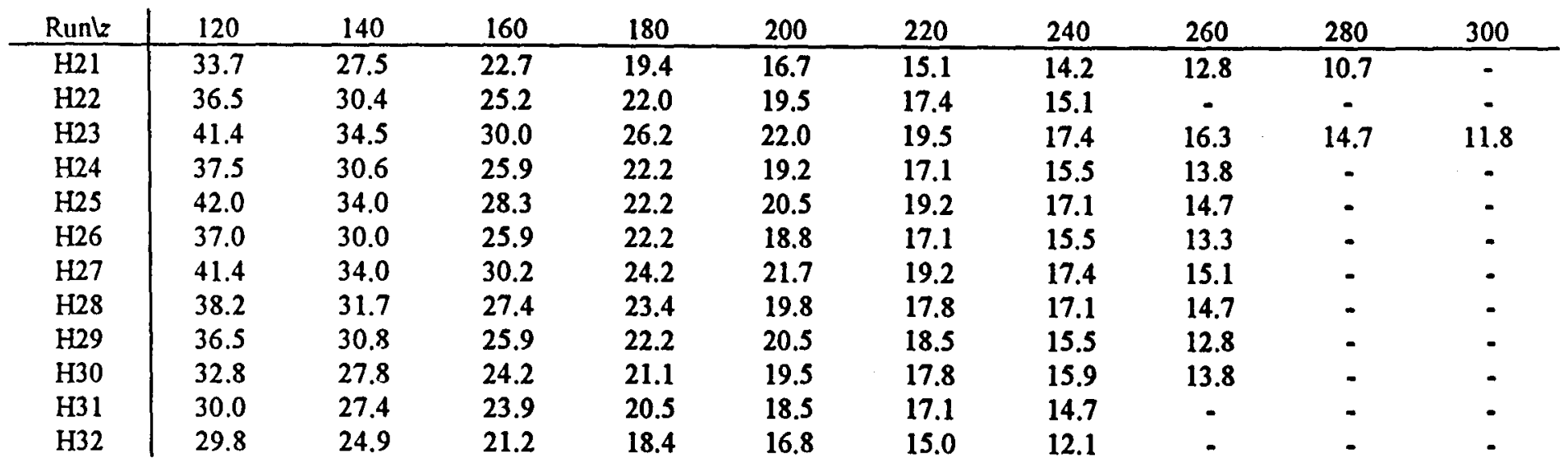




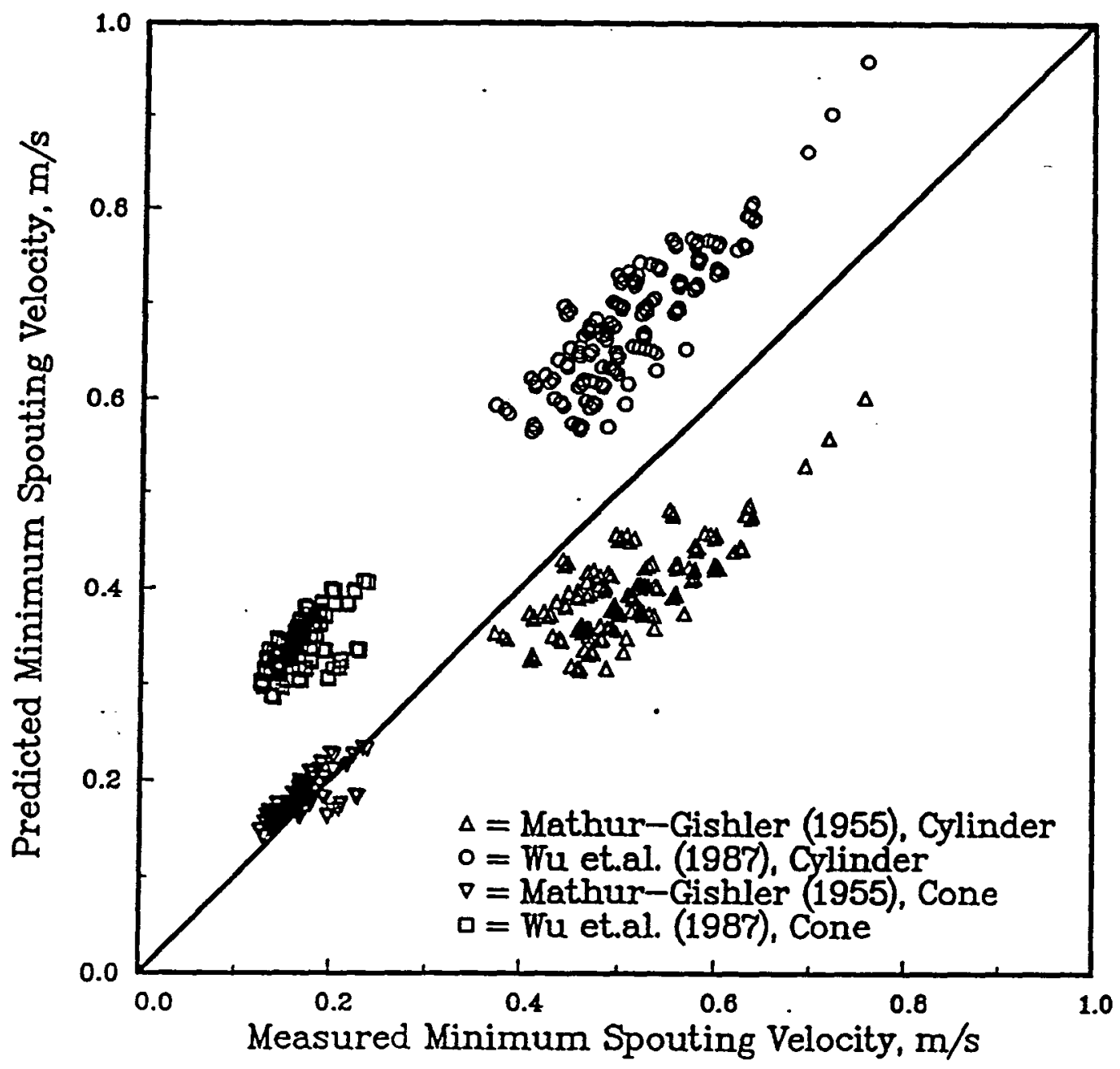

Figure 5.1: Comparison between experimental and predicted $U_{m s}$ values. 
Table 5.5: Fitted constants for $U_{m s}$ correlations.

\begin{tabular}{|c|c|c|c|c|c|c|}
\hline Authors & $k$ & $\alpha$ & $\beta$ & $\gamma$ & $\delta$ & $\begin{array}{c}S_{d} \\
(\mathrm{~m} / \mathrm{s})\end{array}$ \\
\hline Wu et al. (1987) & 10.6 & 1.05 & 0.266 & -0.095 & 0.256 & 0.171 \\
\hline Mathur-Gishler (1955) & 1 & 1 & $1 / 3$ & 0 & 0.5 & 0.092 \\
\hline \multicolumn{7}{|c|}{ Present Work: } \\
\hline All beds ${ }^{a}$ & 6.10 & 1.27 & 0.551 & -0.004 & 0.520 & 0.033 \\
\hline $\begin{array}{l}\text { Conical-Cylindrical } \\
\text { beds onlya }\end{array}$ & 18.5 & 1.19 & 0.373 & -0.193 & 0.263 & 0.030 \\
\hline Conical beds onlya & 0.147 & 0.610 & 0.243 & 0.508 & 0.477 & 0.0095 \\
\hline All beds $b$ & 4.47 & 1.22 & 0.492 & -0.007 & 0.518 & 0.034 \\
\hline All beds ${ }^{c}$ & 13.5 & 1.17 & 0.372 & -0.148 & 0.289 & 0.033 \\
\hline \multicolumn{7}{|c|}{$\begin{array}{l}\text { The diameter of the cylindrical bed is used; }{ }^{b} \text { The maximum annulus diameter }\left(D_{m}\right) \text { is used; } \\
\text { c The diameter is given by } D^{\prime}=0.8 D_{m} \text { for the conical beds and } D^{\prime}=D \text { for the conical- } \\
\text { cylindrical beds. }\end{array}$} \\
\hline
\end{tabular}

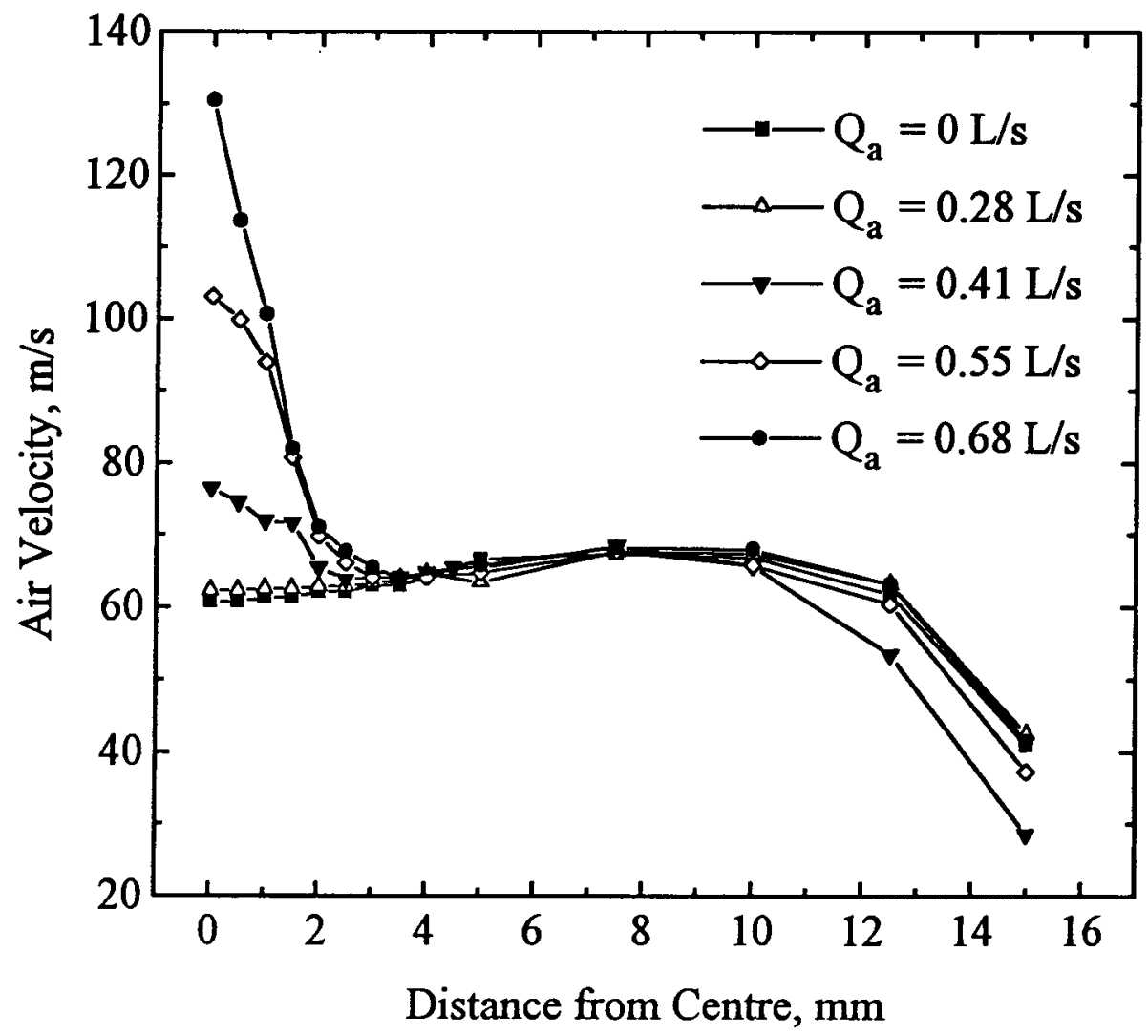

Figure 5.2: Radial velocity profile $10 \mathrm{~mm}$ above the shutter in an empty bed $\left(Q_{s}\right.$ $=27.9 \mathrm{~L} / \mathrm{s}, d_{i}=24.7 \mathrm{~mm}$ and $T=65^{\circ} \mathrm{C}$ ). 
To examine the effect of $Q_{a}$ on $U_{m s}$, two series of experiments were performed with polystyrene particles under the following conditions: $D=0.24 \mathrm{~m}$ and $H=0.35 \mathrm{~m} ; D=0.45 \mathrm{~m}$ and $H=0.31 \mathrm{~m}$. In both cases, the inlet orifice diameter $\left(d_{i}\right)$ was $35 \mathrm{~mm}$. The results obtained with the larger bed are shown in Figure 5.3 and it is apparent that, as $Q_{a}$ was increased, the main air flow rate $\left(Q_{s}\right)$ decreased more rapidly in order to achieve minimum spouting. As a result, $U_{m s}$ decreased with $Q_{a}$. The total momentum flow into the beds, which results from $Q_{a}$ and $Q_{s}$, was therefore calculated as

$$
M_{t}=\frac{4 \rho Q_{s}^{2}}{\pi d_{o}^{2}}+\frac{4 \rho Q_{a}^{2}}{\pi d_{a t}^{2}}
$$

where $d_{a t}$ denotes the diameter of the nozzle tip through which the atomizing air is discharged (see Figures 3.7 and 3.13). As can be seen from Figure 5.4, the total momentum flow at minimum spouting is approximately constant. Since Equations (2.2) and (2.3) do not account for the momentum introduced into the bed by the atomizing air, $Q_{a}$ was incorporated into the equations by calculating a normalized inlet diameter $\left(d_{i}^{\prime}\right)$ given by

$$
d_{i}^{\prime 2}=4 \rho\left(Q_{s}+Q_{a}\right)^{2} / \pi M_{t}
$$

The total momentum flux $\left(M_{t}\right)$ is found from Equation (5.1).

Since Equations (2.2) and (2.3) were developed primarily for conical-cylindrical beds, experiments were undertaken to examine $U_{m s}$ as a function of bed height for shallow beds where the particles are restricted to a portion of the conical section only. The experiments were conducted with polystyrene particles under the following conditions: $d_{i}=25 \mathrm{~mm}, Q_{a}$ $=0.53 \mathrm{~L} / \mathrm{s}, T=60^{\circ} \mathrm{C}$. The results are shown in Figure 5.5 and indicate that when the bed is confined to the conical section, $U_{m s}$ is not proportional to $H^{0.5}$ as suggested by the Mathur-Gishler equation, but it is approximately proportional to $H$. More specifically, Table 5.5 indicates that $U_{m s} \propto H^{1.08}$ for conical beds. The $H^{0.5}$ relationship suggested by the Mathur-Gishler equation predicts higher $U_{m s}$ values than are found experimentally for shallow conical beds. The reason is that, in reality, more air flows through the spout than 


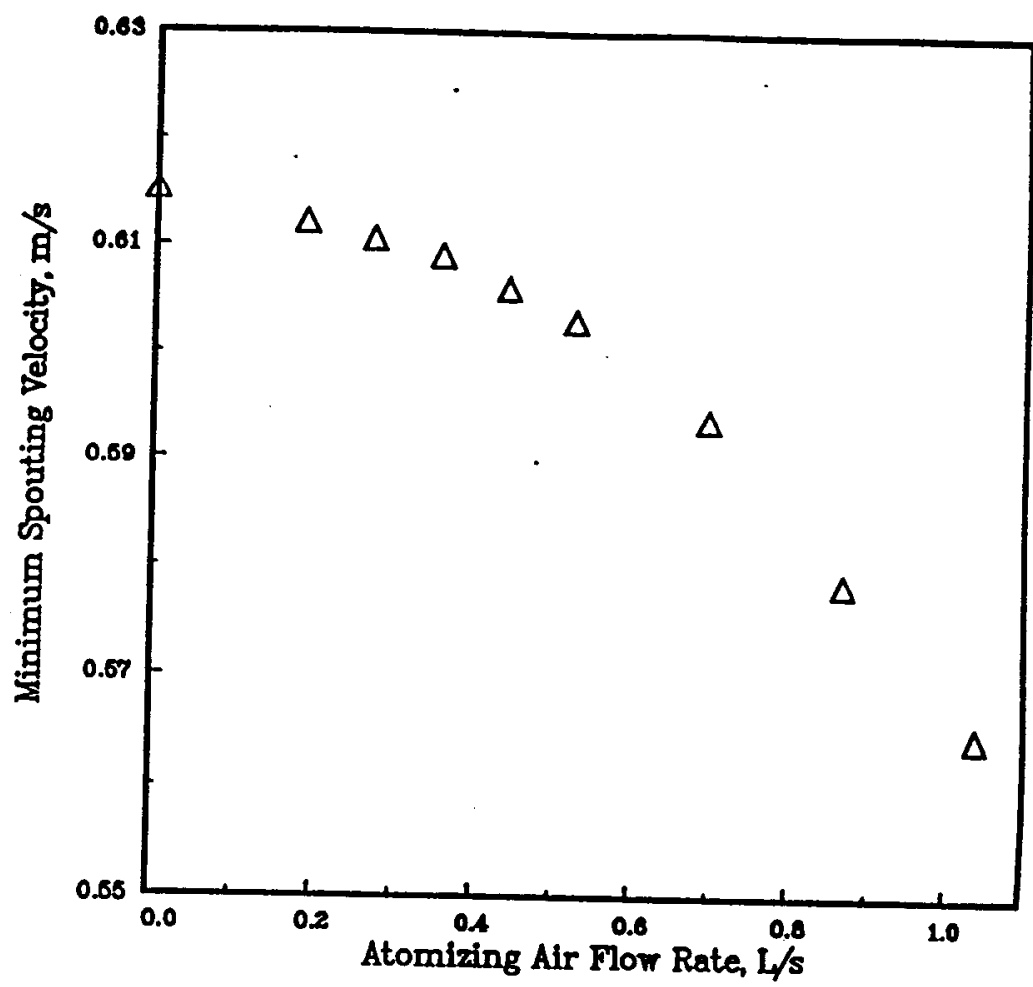

Figure 5.3: Effect of atomizing air on minimum spouting velocity (minimum spouting velocity is based only on the main spouting air flow rate; $D=0.45 \mathrm{~m}, H=$ $0.31 \mathrm{~m}, d_{i}=35 \mathrm{~mm}$ ).

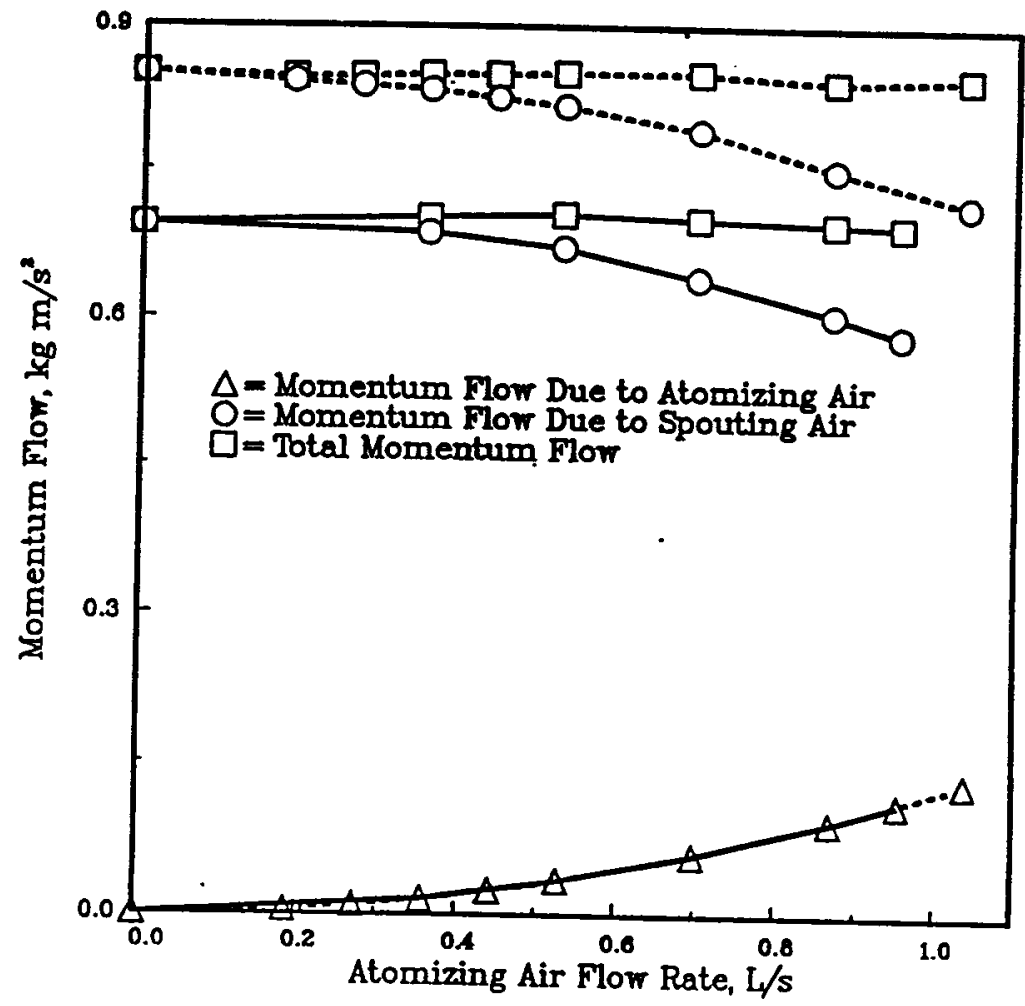

Figure 5.4: Momentum flow of air into the spouted bed (dashed and solid lines represent the $0.24 \mathrm{~m}$ and $0.45 \mathrm{~m}$ dia. beds, respectively). 


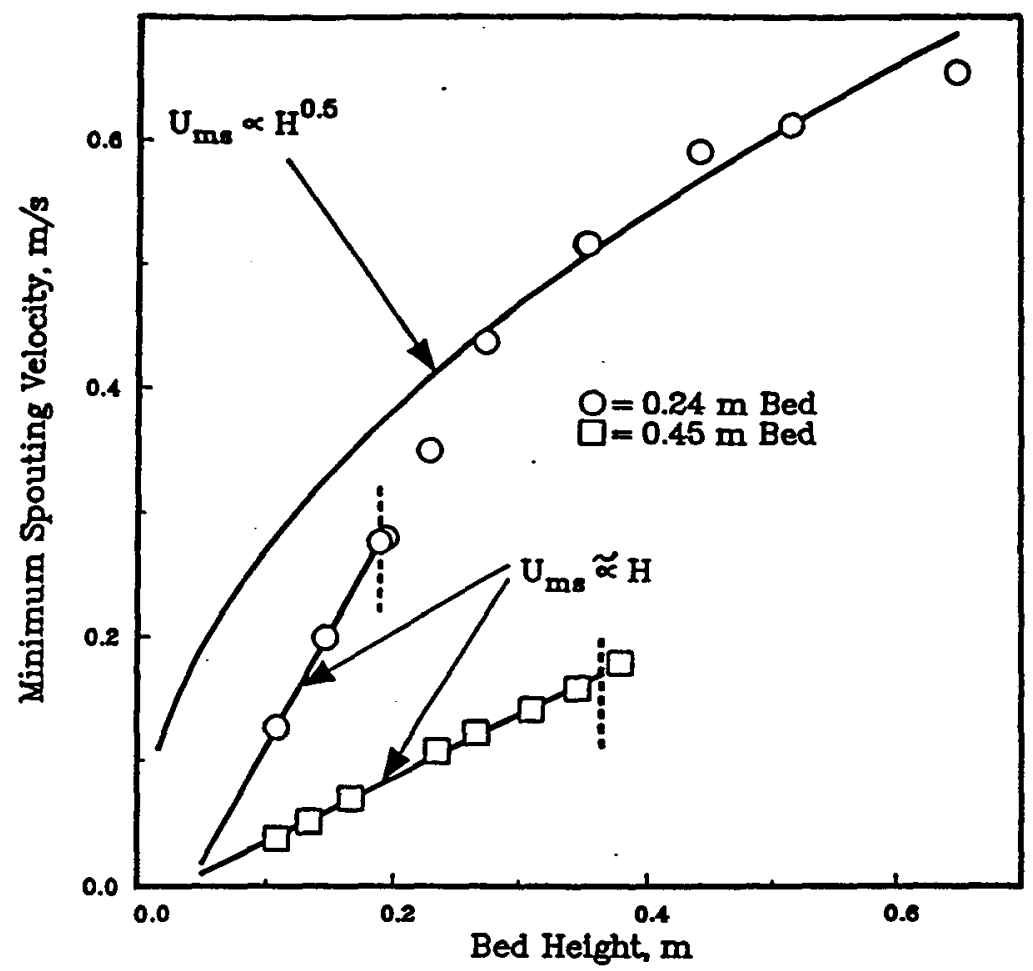

Figure 5.5: Effect of bed height on minimum spouting velocity, $U_{m s .}$ (Cone-cylinder junctions are denoted by dashed lines; solid lines represent indicated relationships fitted to experimental data).

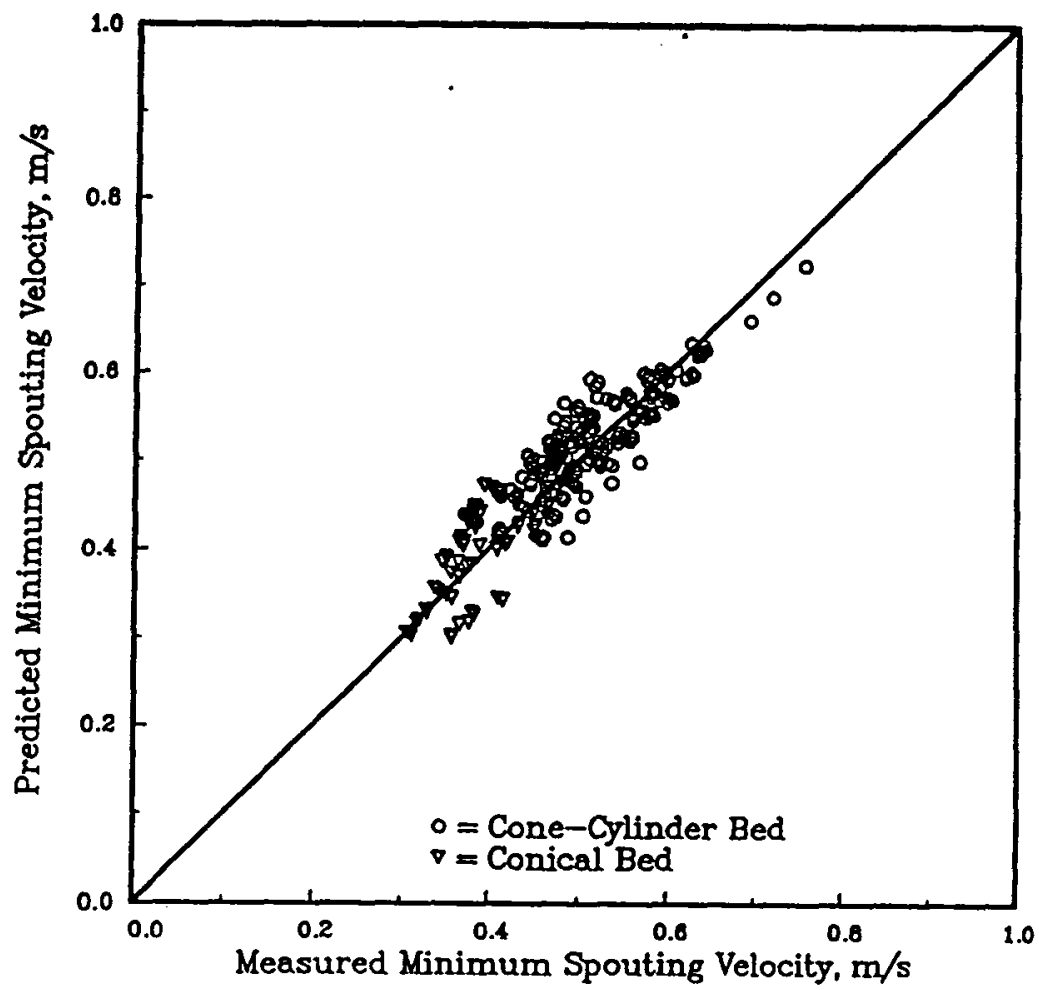

Figure 5.6: Comparison between experimental data and predictions from correlation based on the optimum diameter $D^{\prime}$. 
implied by this equation because the inlet air is unable to spread into the annulus as effectively since the annulus is smaller and the wall is closer to the centre of the bed.

Since the constants obtained for the Wu et al. equation are different in the case of conical and conical-cylindrical beds, an attempt was made to unify the results by using the maximum bed diameter $\left(D_{m}\right)$ covered by the particles. When the bed particles extend into the cylindrical region of the spouted bed, this diameter corresponds to the cylinder diameter. The coefficients resulting from the least squares fit are presented in Table 5.5.

Upon observing the solids movement in the conical beds, $D_{m}$ was suspected to be an inappropriate variable representing $U_{m s}$. The bed particles near the wall on the surface of the annulus were observed to move significantly slower than the particles near the centre of the bed. These differences in particle velocities were much more pronounced in conical beds than in conical-cylindrical beds. Consequently, the conical beds were suspected to behave as conical-cylindrical beds with $D<D_{m}$.

To determine the "effective" bed diameter $(D)$ representing $U_{m s}$ for shallow, conical beds the following relationship was considered:

$$
D^{\prime}=c D_{m}
$$

The proportionality constant $(c)$ was determined by obtaining the best fit between the experimental and predicted results. The optimum value of $c$ was found to be 0.8 and the corresponding coefficients in the Wu et al. equation are also given in Table 5.5. As shown by Figures 5.1 and 5.6, the agreement between predicted and experimental results is improved. The optimal, modified Wu et al. equation thus becomes:

$$
U_{m s}^{\prime}=13.5 \sqrt{2 g H}\left(\frac{d_{p}}{D^{\prime}}\right)^{1.17}\left(\frac{d_{i}^{\prime}}{D^{\prime}}\right)^{0.372}\left(\frac{H}{D^{\prime}}\right)^{-0.148}\left(\frac{\rho_{p}-\rho}{\rho}\right)^{0.289}
$$

It is interesting to note that Equation (5.4) implies that $U_{m s}^{\prime} \propto H^{0.352}$ for deep beds. The corresponding exponents of $H$ for the equations of $\mathrm{Wu}$ et al. (1987) and Mathur and 
Gishler (1955) are 0.4 and 0.5 , respectively. This result also supports the contention made by Wu et al. that the $H$ and $\rho$ terms should not be combined as suggested by Mathur and Gishler. For shallow conical beds where $D^{\prime} \propto H$, Equation (5.4) reduces to $Q_{m s}(=\pi / 4$ $\left.\cdot U_{m s}^{\prime}(D)^{2}\right) \propto H^{0.96}$, i.e., the relationship is virtually linear. This agrees well with the experimental data shown in Figure 5.5 (note that $U_{m s}$ is based on the column diameter in this figure; hence, $\left.Q_{m s} \propto U_{m s}\right)$. The conical bed data also could be well represented by the Wan-Fyong et al. (1969) equation, corrected for atomizing air (see Appendix I).

\subsubsection{Axial Pressure Profile in Annulus}

The axial pressure profiles near the spout-annulus interface are given in Table 5.3. The temperature, atomizing air and spouting air flow rates did not have a significant effect on the pressure profiles. Furthermore, the variations in the radial pressure profiles were small for conical-cylindrical and conical beds (see, for example, Figure 5.7).

The axial pressure profiles for two beds are shown in Figures 5.8 and 5.9 together with predictions based on the equations provided by Epstein-Levine (1980), Rovero et al. (1983) (which is the Epstein-Levine equation modified for beds with a conical base), and Morgan and Littman (1980). $U_{m f} U_{b} U_{a H m}$ and $H_{m}$ in these equations were calculated from the correlations given by Grace (1982), Clift et al. (1978), Epstein et al. (1978) and McNab and Bridgwater (1977), respectively. The first two expressions under-predicted the axial pressure profile whereas the Morgan-Littman equation gave fairly good agreement in the case of conical-cylindrical beds (see Figure 5.8). By contrast, all three equations performed poorly for conical beds as shown by Figure 5.9. However, when the equations are modified by using $D^{\prime}$ (see Equation (5.3)), the agreement is significantly improved, especially in the case of the Morgan-Littman equation (see Figure 5.10).

Further examination of the Morgan-Littman correlation showed that for the deeper bed $(0.53 \mathrm{~m})$ and changes in $d_{i}$, the agreement was not as good. In general, the correlation 


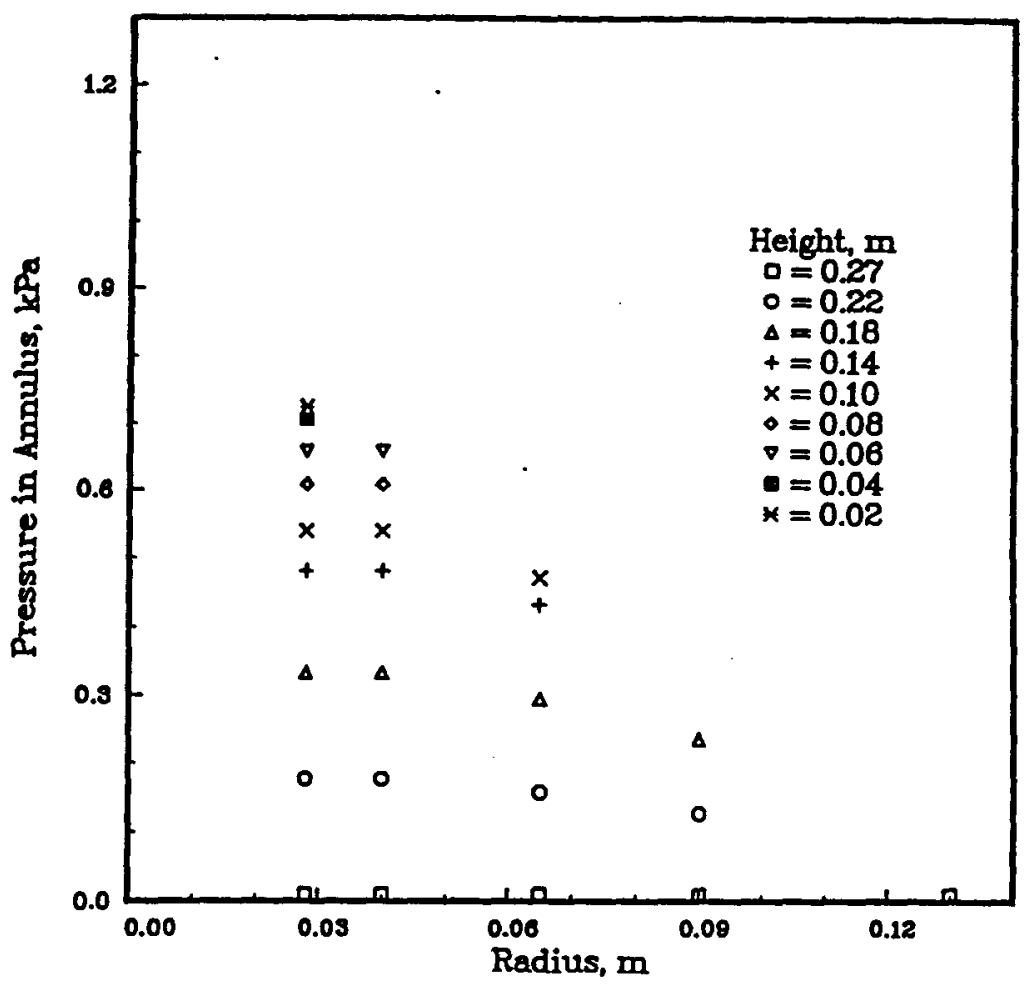

Figure 5.7: Pressure profile in the annulus (conical bed, Run H22).

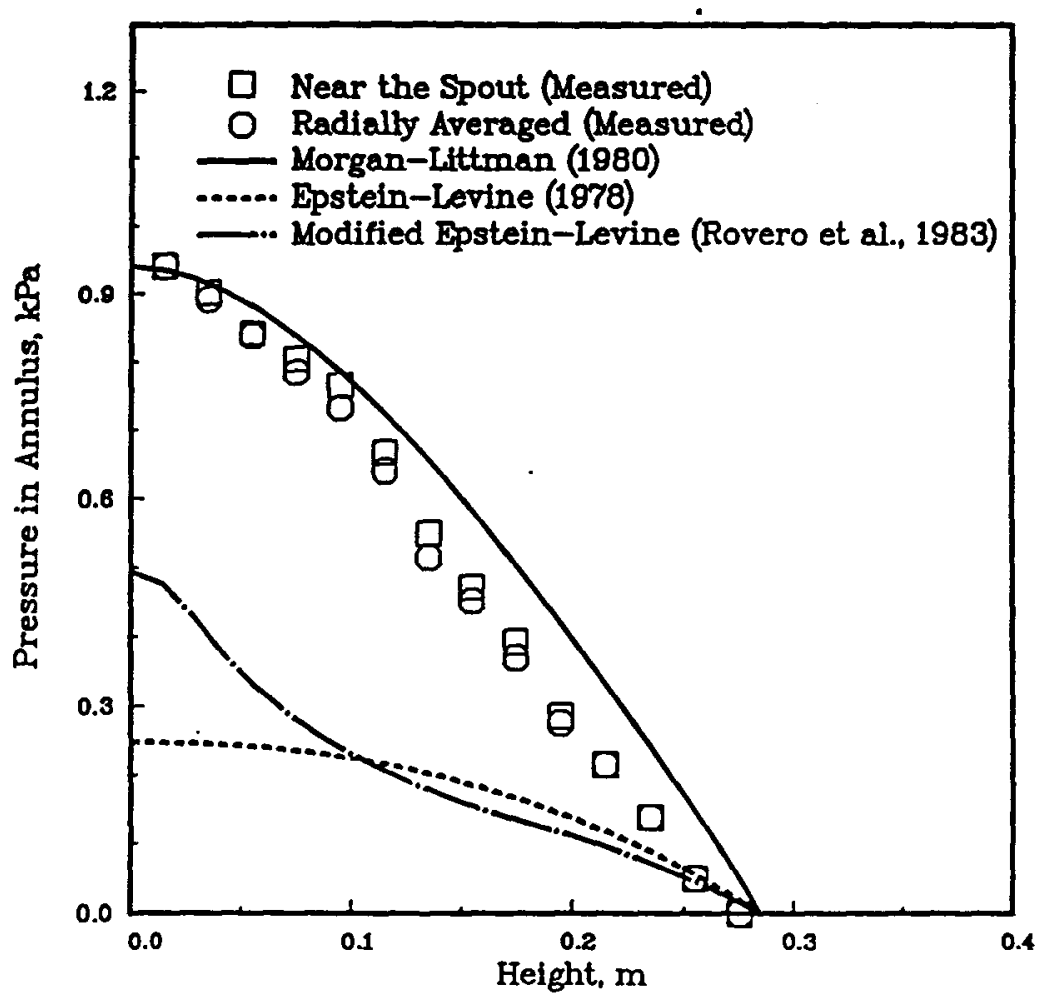

Figure 5.8: Axial pressure profile in the annulus near the spout-annulus interface (conical-cylindrical bed, Run $\mathrm{Hl}$ ). 


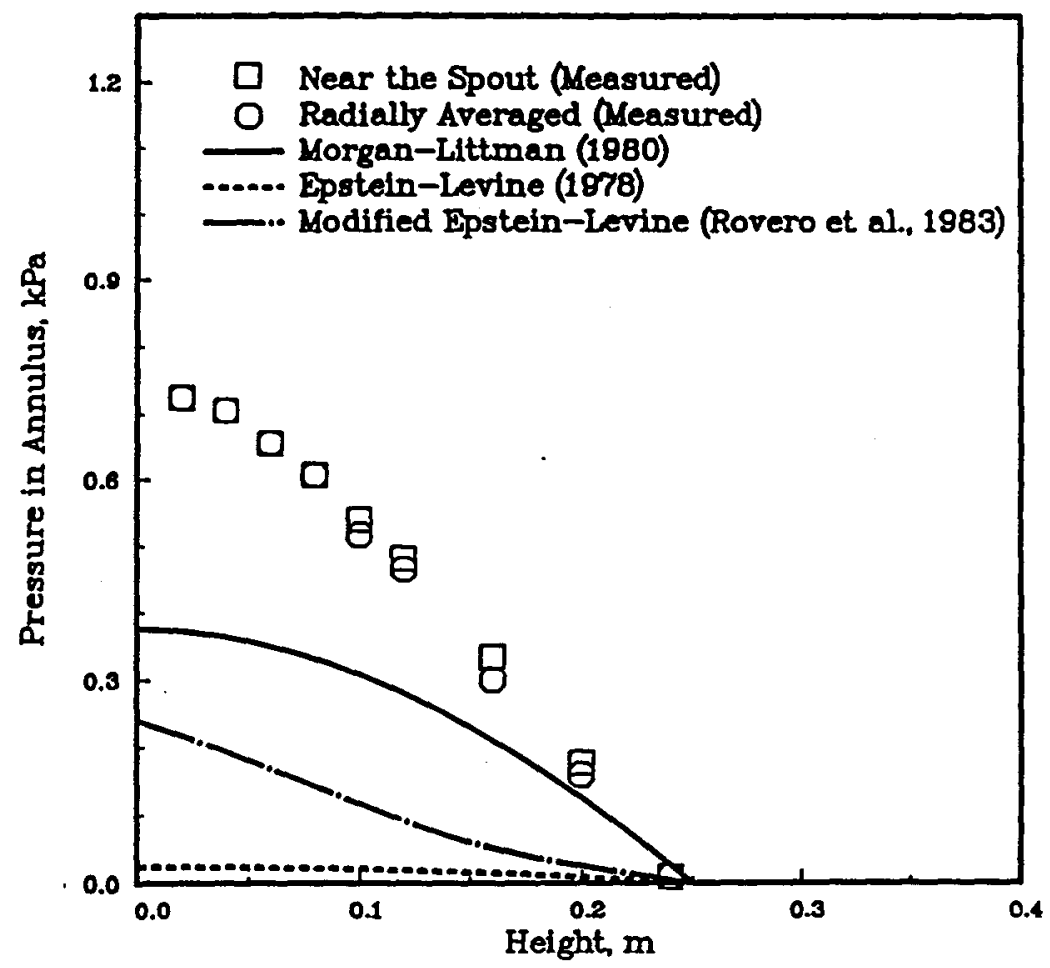

Figure 5.9: Axial pressure profile in the annulus (conical bed, Run H22, column diameter used in calculation).

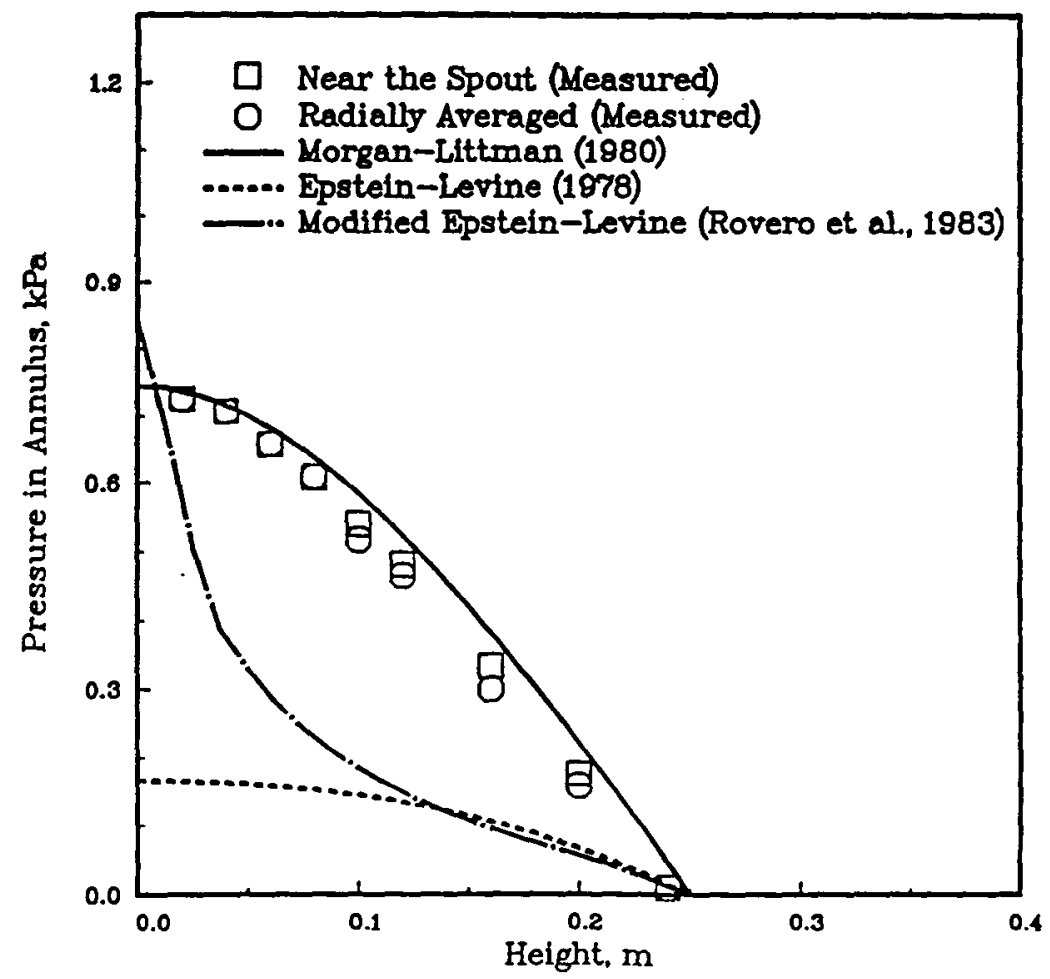

Figure 5.10: Axial pressure profile. in the annulus (conical bed, Run H22, $D^{\prime}$ used in calculation). 


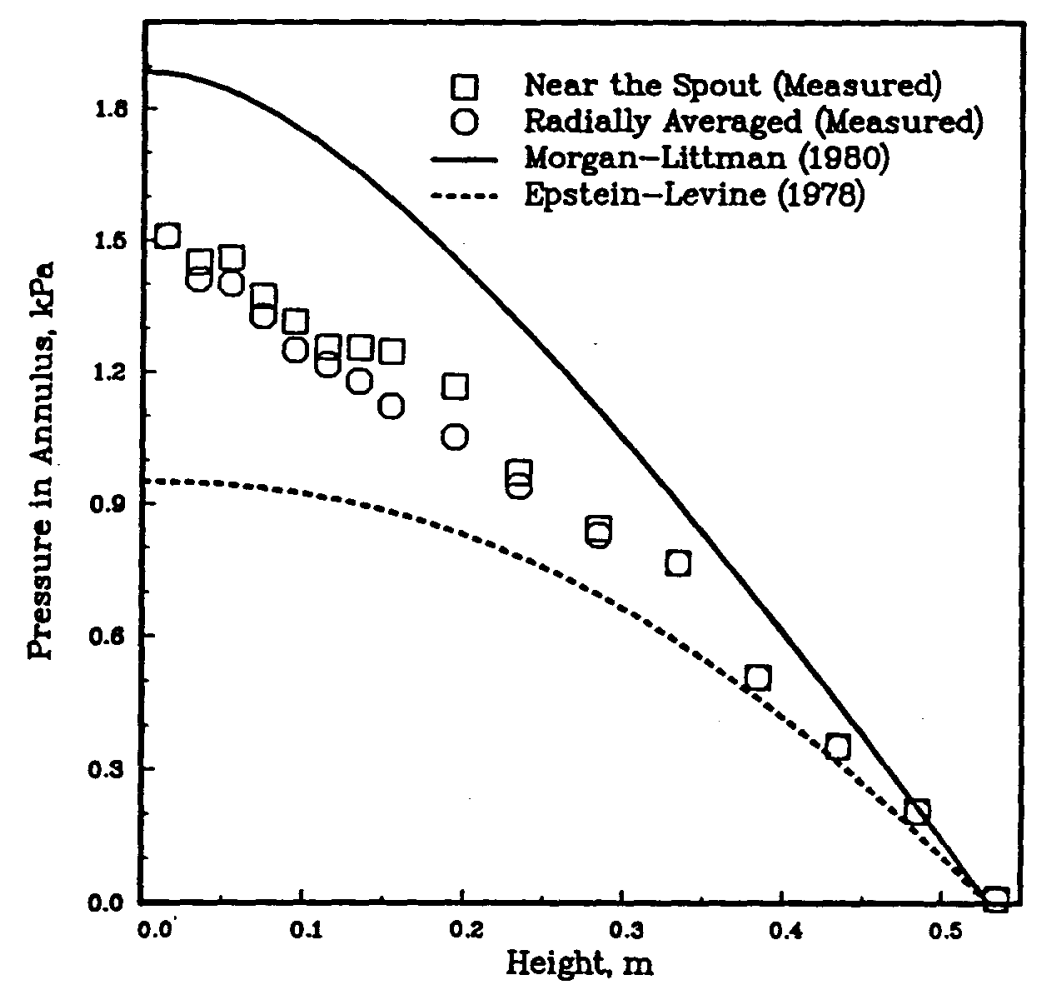

Figure 5.11: Axial pressure profile in the annulus $(H=0.53, \operatorname{Run} H 17)$.

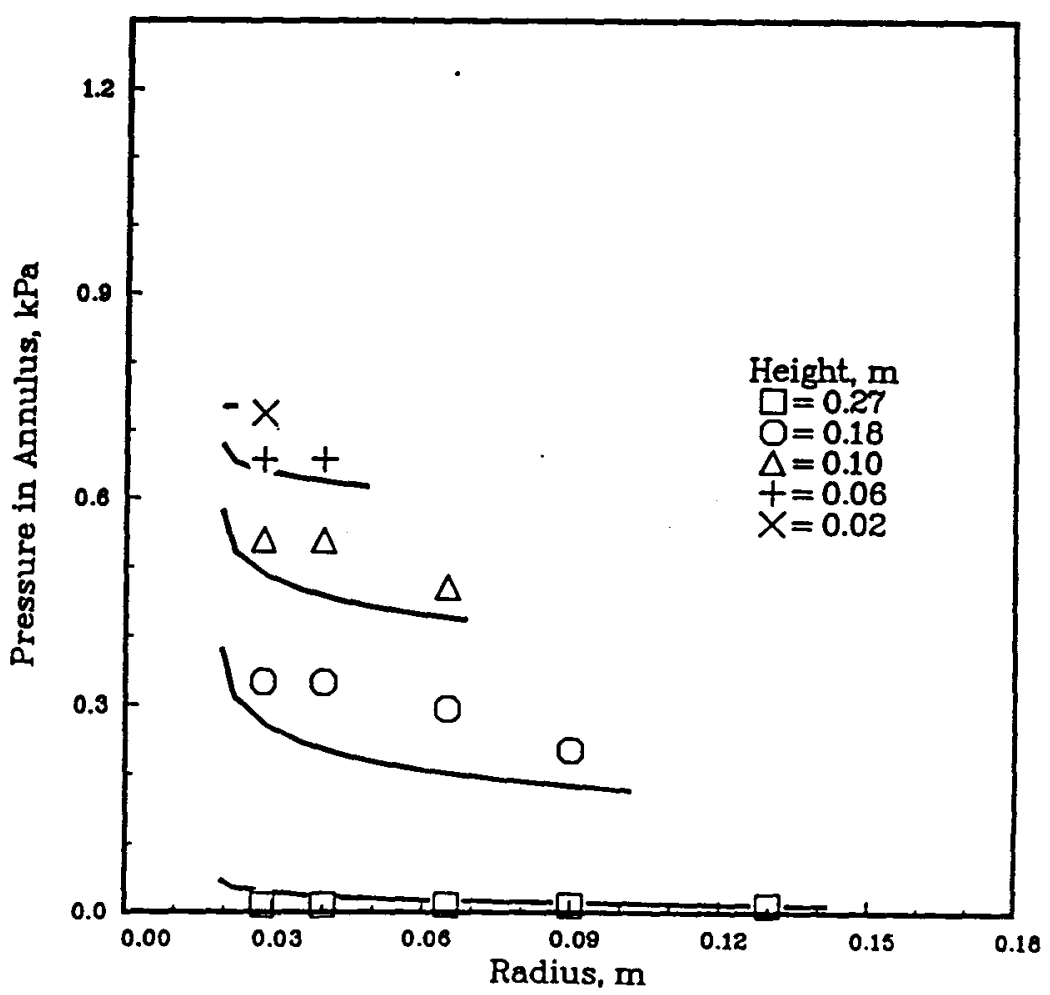

Figure 5.12: Comparison between measured and predicted pressure profile in the annulus. 
over-estimated the pressure drop in the $0.53 \mathrm{~m}$ bed (see Figure 5.11), and under-estimated the effect of $d_{i}$. In an attempt to improve the predictions, the optimal constants in Equation (2.14) for the $0.24 \mathrm{~m}$ bed data were determined by a least squares technique. Although the optimal constants improved the agreement for deeper beds, the predictions for the shallower beds were somewhat impaired.

\subsubsection{Velocity Profile in Annulus}

The radial pressure variation in the annulus was found to be small (see Figure 5.7). However, even small pressure gradients can lead to significant changes in the air flow rate in the annulus. If no radial variation of pressure is assumed and the Morgan-Littman correlation is used, significant errors in the air flow rate result. This result is due to the larger flow area away from the centre of the bed. For this reason, the vector Ergun (1952) equation is solved to determine the fluid flow in the annulus.

The solution of the vector Ergun equation for Run $\mathrm{H} 22$ is shown in Figure 5.12 and the method of solution is given in Appendix I. The model predictions, in general, were in good agreement with the measured values. Moreover, significant improvement to the spout velocity predictions was observed when the fluid flow in the annulus is calculated using the vector Ergun equation rather than the one-dimensional equation.

\subsubsection{Axial Velocity Profile in Spout}

The measured spout velocity profiles are given in Table 5.4. The effect of atomizing air flow on $u_{s}$ is significant and is particularly noticeable in the lower section of the bed as shown by Figure 5.13. The total spouting air flow exhibits a similar effect on $u_{s}$. Changes in bed height did not greatly influence the velocity profile in the spout.

The spout velocities predicted by the mass and momentum balance equations (Lefroy and Davidson, 1969) introduced in Chapter 4 represented the measured velocities well (e.g., 


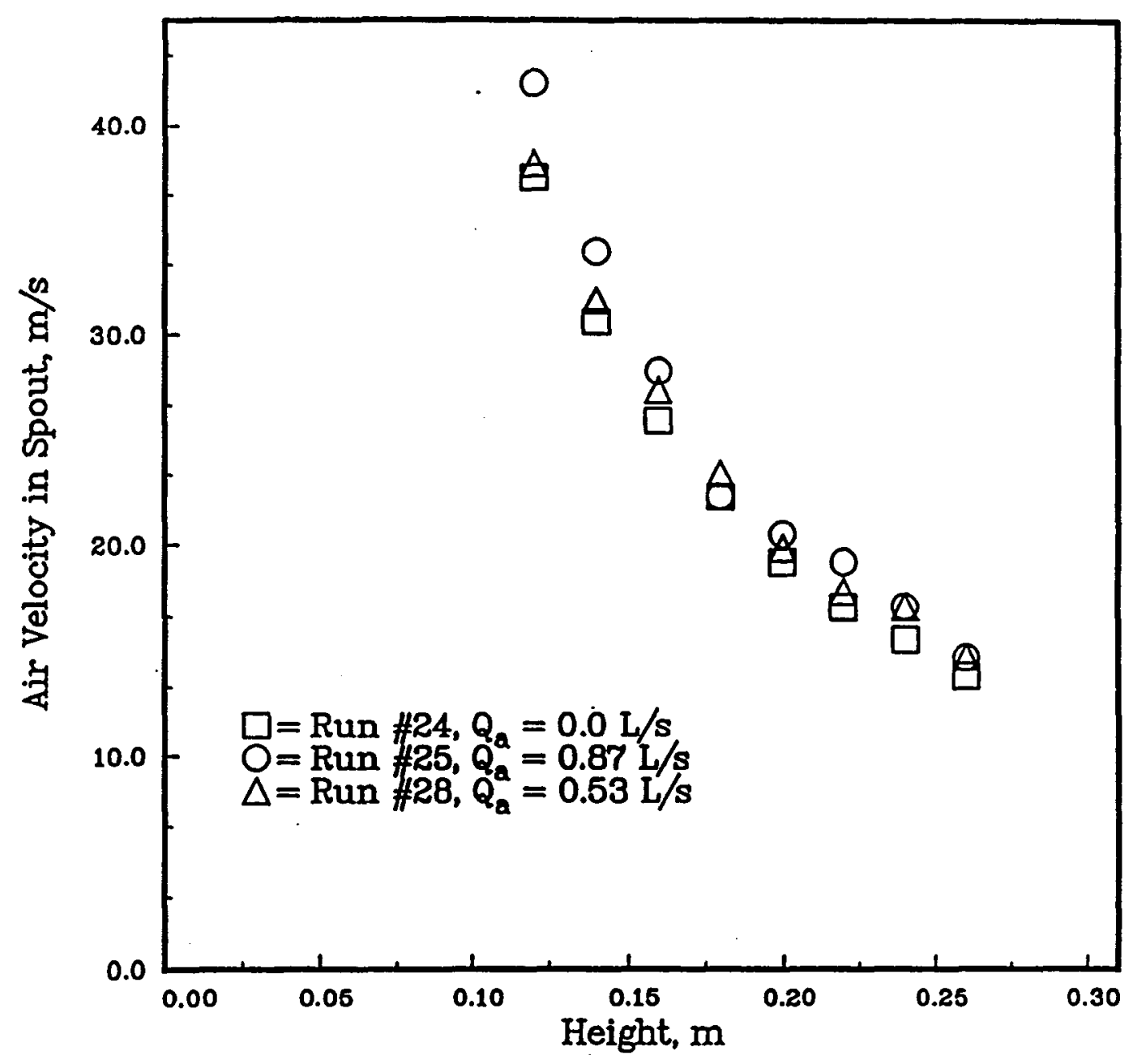

Figure 5.13: Effect of atomizing air on axial air velocity profile in the spout $\left(Q_{s}\right.$ is fixed at approximately $32 \mathrm{~L} / \mathrm{s}$ in all runs). 


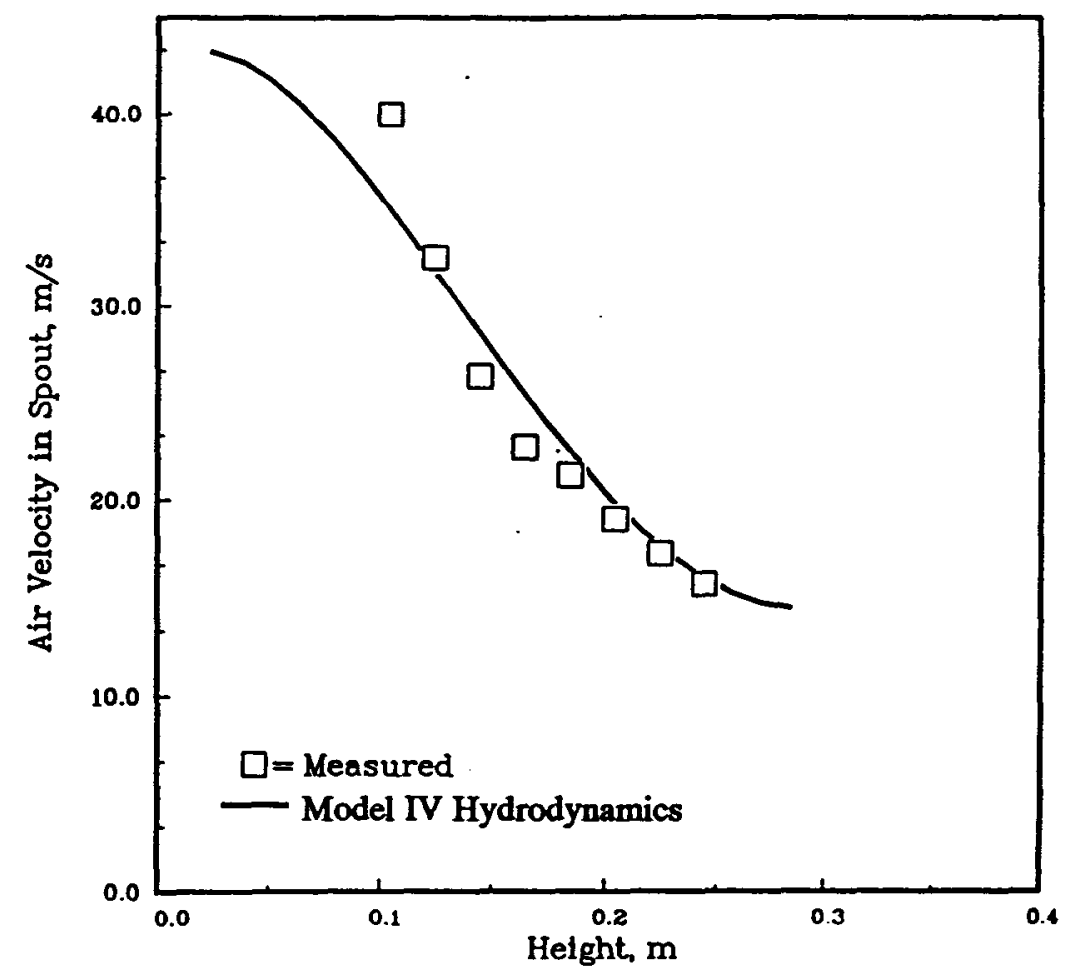

Figure 5.14: Air velocities in the spout determined experimentally and from Model IV hydrodynamics (Run H1).

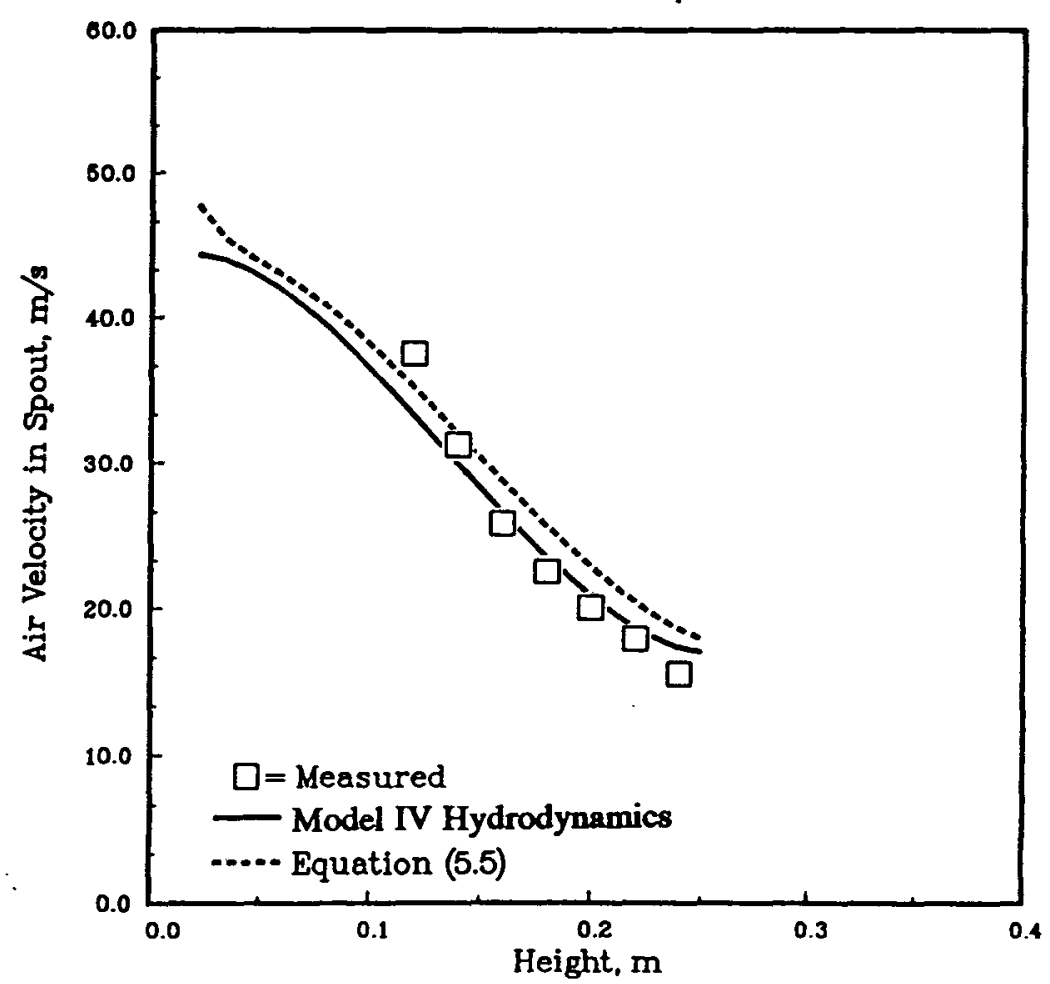

Figure 5.15: Air velocities in the spout determined experimentally, from Model IV hydrodynamics and from Equation (5.5)(Run H22). 
see Figures 5.14 and 5.15) if the aforementioned modified equations were used. The correlations and assumptions used in the mass and momentum balance equations included:

- The spout diameter is constant and is given by the Wu et al. correlation (1987);

- The pressure profile is given by the Morgan-Littman (1980) equation modified for conical beds and effective bed diameter $D^{\prime}$;

- The air flow rate in the annulus is given by the vector Ergun (1952) equation;

- The fluid-particle interaction term proposed by Richardson and Zaki (1954) is valid;

- The spouting air flow rate is calculated from the total momentum flux into the bed rather than the total flow rate,

- The air density is based on the initial spouting air temperature rather than the average bed temperature.

A sensitivity analysis for these variables showed that the strongest factor influencing the agreement between the velocity predictions and measurements was the correlation used to predict the pressure profile; good agreement with the velocity measurements was observed when the difference between the predicted and measured pressures was small.

Figures 5.14 and 5.15 show more pronounced deviations between the measured and predicted velocities near the base of the bed. Regardless of the presence of the atomizing air, the measured velocity was expected to be higher than the predicted (i.e., average) velocity since the measurements were based on the highest pressure difference indicated by the pitot tube (which corresponds to the highest velocity). The large discrepancy near the inlet may be the result of a large radial velocity gradient in the spout (Krzywanski, 1992; Abramovich, 1963).

Moreover, the velocity profiles shown in Figures 5.2 and 5.13 suggest considerable influence of the atomizing air on the radial velocity profiles, especially near the base of the bed. In an attempt to model the atomizing air velocity, the following equations were developed, 
based on the assumptions that no mixing between the atomizing jet and spouting air occurred and that the solids voidage remained the same as the average voidage in the spout:

$$
\mathrm{d}\left(\varepsilon_{s} u_{a s} A_{a s}\right) / \mathrm{d} z=0
$$

where $A_{a s}=\pi D_{a s}^{2} / 4$

and $\quad \mathrm{d} D_{a s} / \mathrm{d} z=k_{\theta}\left|u_{a s}-u_{s}\right| /\left(\left|u_{a s}\right|+\left|u_{s}\right|\right)$

Equation (5.7) is given in Abramovich (1963). $D_{a s}$ represents the spread of the jet boundary layer and $k_{\theta}$ represents the fully developed jet angle in still air. Using the spray angle $\left(k_{\theta}\right)$ value of $20^{\circ}$, Equation (5.5) was solved together with Equations (4.27) to (4.30). The results are presented in Figure 5.15 and show that the atomizing air velocity changes rapidly to a value slightly higher than the spouting air velocity a short distance away from the base of the bed. Above this height, the atomizing air velocity changes at the same rate as the spouting air velocity. This result is most likely due to the assumption that no mixing occurs across the jet boundary layer. In reality, mixing across the atomizing air jet boundary should occur (Rushton and Oldshue, 1953; Abramovich, 1963) especially with the entrainment of solids into the spout, and consequently, the boundary should quickly disappear. To model the atomizing air velocity in the spout appropriately, a means of estimating the mixing length should be devised; however, this task was beyond the scope of this thesis and is pursued no further.

Other explanations for the discrepancy in the measured and predicted velocities near the base of the bed include the constant spout diameter assumption and the inability to predict the pressure peak by the Morgan-Littman correlation. Most spout shapes quickly diverge to a constant spout diameter ( $\mathrm{Lim}, 1978$ ); therefore, the gas velocities in the lower spout region will be higher than if a constant spout diameter is assumed. In most of the pressure measurements (see Table 5.3), a pressure peak occurred near the base of the bed and none of the correlations given in the literature predicts such behavior. It is very likely that the 
inability to predict the pressure peak by the correlation used in this work resulted in a lower predicted spout velocity near the base of the bed.

\subsubsection{Solids Movement}

The claims that particle segregation occurs in the annulus were made by Kutlouglu et al. (1983), Cook and Bridgewater (1978), Robinson and Waldie (1978), and Piccinini et al. (1977). The claims were visually investigated by using sulfur coated urea particles. Using a batch of screened particles of size less than $1.17 \mathrm{~mm}$ and greater than $3.35 \mathrm{~mm}$ (using US \# 16 and \# 6 sieves) dyed blue and red, respectively, the movement of the tagged particles in a larger batch of sulfur coated urea particles was observed in a six inch half-column. The urea used for this experiment was a low grade urea, and contained $10 \%$ by mass (after coating) of particles with $d_{p}>3.35 \mathrm{~mm}$ and $25 \%$ with $d_{p}<1.17 \mathrm{~mm}$. Inspection of the these particles spouting at various bed heights and air flow rates using a video camera showed no observable particle segregation in the annulus.

Observations of the solids also indicated that the solids near the spout-annulus interface moved faster than the solids near the wall; this is consistent with the findings by Rovero et al. (1985).

\subsection{Spray Studies}

The operating ranges examined are summarized in Table 5.6. The drop size data detected by the image analyzer are extensive and are included in Appendix II.

\subsubsection{Operating Limits}

As stated in Chapter 3, the operating range of the atomizing nozzle used in this study was limited by no atomization and inconsistent atomization at low and high atomizing air flow 
Table 5.6: Operating ranges of the spray studies.

\begin{tabular}{|l|c|}
\hline \multicolumn{2}{|c|}{ Fixed Operating Conditions } \\
\hline Atomizing Air Temperature & $160^{\circ} \mathrm{C}$ \\
\hline Auxiliary (Spouting Air) Temperature & $60^{\circ} \mathrm{C}$ \\
\hline Steam Pressure & $75-80 \mathrm{psi}$ \\
\hline Molten Sulfur Temperature & $\sim 150^{\circ} \mathrm{C}$ \\
\hline \multicolumn{2}{|c|}{ Varied Operating Conditions } \\
\hline Atomizing Air Flow Rate & 0.23 to $0.70 \mathrm{~L} / \mathrm{s}$ \\
\hline Sulfur Flow Rate & 2.1 to $6.1 \mathrm{~g} / \mathrm{s}$ \\
\hline
\end{tabular}

rates, respectively. Both limits can be seen in Figure 5.16. The lower limit of the atomization velocity $\left(U_{m a}\right)$ could be well represented by

$$
U_{m a}=13.8+1.61 W_{s}
$$

where $W_{s}$ and $U_{m a}$ have units of $\mathrm{kg} / \mathrm{h}$ and $\mathrm{m} / \mathrm{s}$, respectively. The upper limit of consistent atomization or the minimum pulsating velocity $\left(U_{m p}\right.$, in $\left.\mathrm{m} / \mathrm{s}\right)$ obeyed the following linear relationship for $W_{s}>8 \mathrm{~kg} / \mathrm{h}$ :

$$
U_{m p}=3.88+4.59 \mathrm{~W}_{s}
$$

$U_{m a}$ corresponds to the minimum energy required to atomize sulfur and Equation (5.8) should be valid for most fluid atomizers. $U_{m p}$ corresponds to the maximum atomization energy, i.e. additional air is wasted. This upper limit is probably caused by the pressure feed system used in this work and may not apply if other feed systems such as a sulfur pump were used.

The atomizing air velocity used in the coating runs was kept approximately mid-way between the operating limits to ensure that the spray was properly developed. 


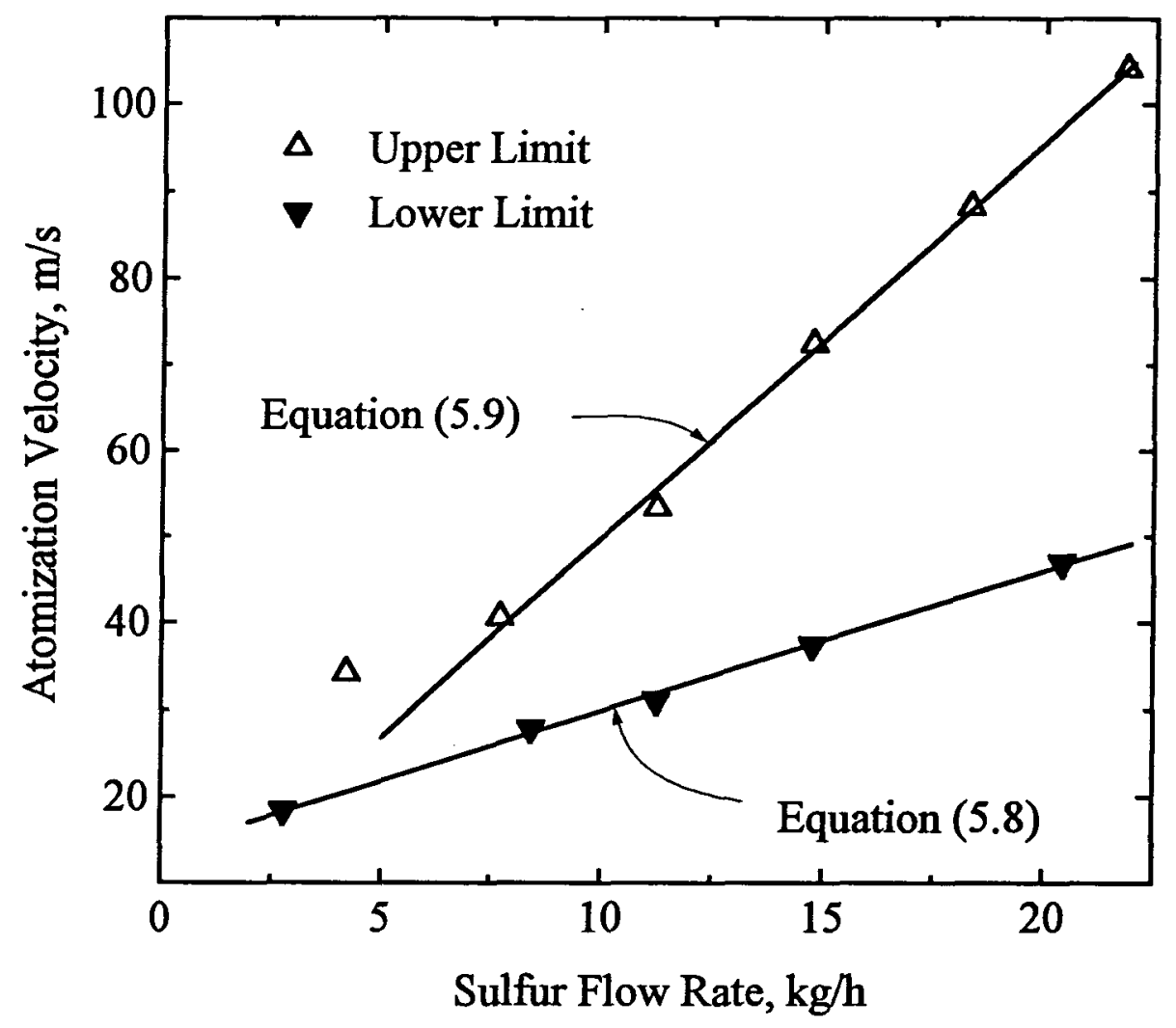

Figure 5.16: Operating limits for atomization of sulfur.

\subsubsection{Spray Drop Size Distribution and Average Drop Size}

A typical sulfur droplet size distribution detected by the image analyzer is shown in Figure 5.17. The majority of droplets fell into the size range of 6 to $50 \mu \mathrm{m}$ and appeared to follow a log-normal distribution (Orr, 1966), i.e.,

$$
N=\frac{\Sigma N}{\ln \sigma_{g} \sqrt{2 \pi}} \exp \left(\frac{-\left(\ln d_{s}-\ln d_{g c}\right)^{2}}{2 \ln ^{2} \sigma_{g}}\right)
$$

where $N=$ frequency of observation of the spray droplet diameter $d_{s}$

$$
\begin{aligned}
& \ln d_{g c}=\Sigma\left(N \ln d_{s}\right) / \Sigma N \\
& \ln \sigma_{g}=\sqrt{\Sigma\left[N\left(\ln d_{s}-\ln d_{g c}\right)^{2}\right] / \Sigma N}
\end{aligned}
$$




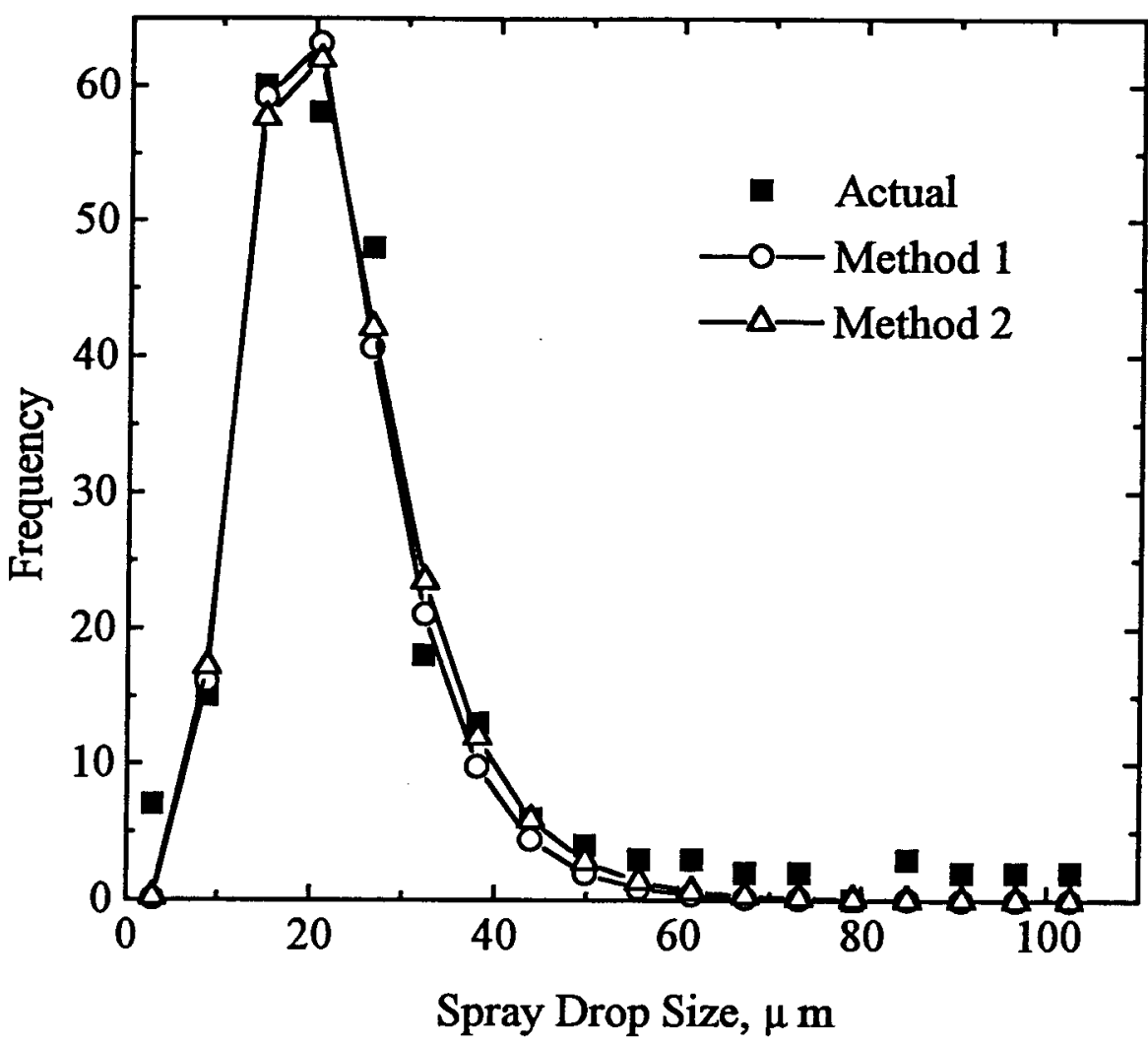

Figure 5.17: Experimental drop size distribution and predictions using lognormal equation (Run S1a).

A log-normal plot is shown in Figure 5.18. The Nukiyama-Tanasawa distribution (Lewis et al., 1948) gave a good representation of the larger drop sizes (see Figure 5.19), but failed for the range 6 to $50 \mu \mathrm{m}$ where the highest frequencies of drops were recorded. The Nukiyama-Tanasawa equation is given by

$$
\ln \left(\frac{N}{d_{s}^{2} \Delta d_{s}}\right)=\ln k_{1}-k_{2} d_{s}^{k_{3}}
$$

where $\Delta d_{s}$ denotes the diameter range of the $N$ droplets. The log-normal distribution was therefore used to represent the drop size data, and the mean drop size and the deviation were calculated from Equations (5.11) to (5.12), respectively. 


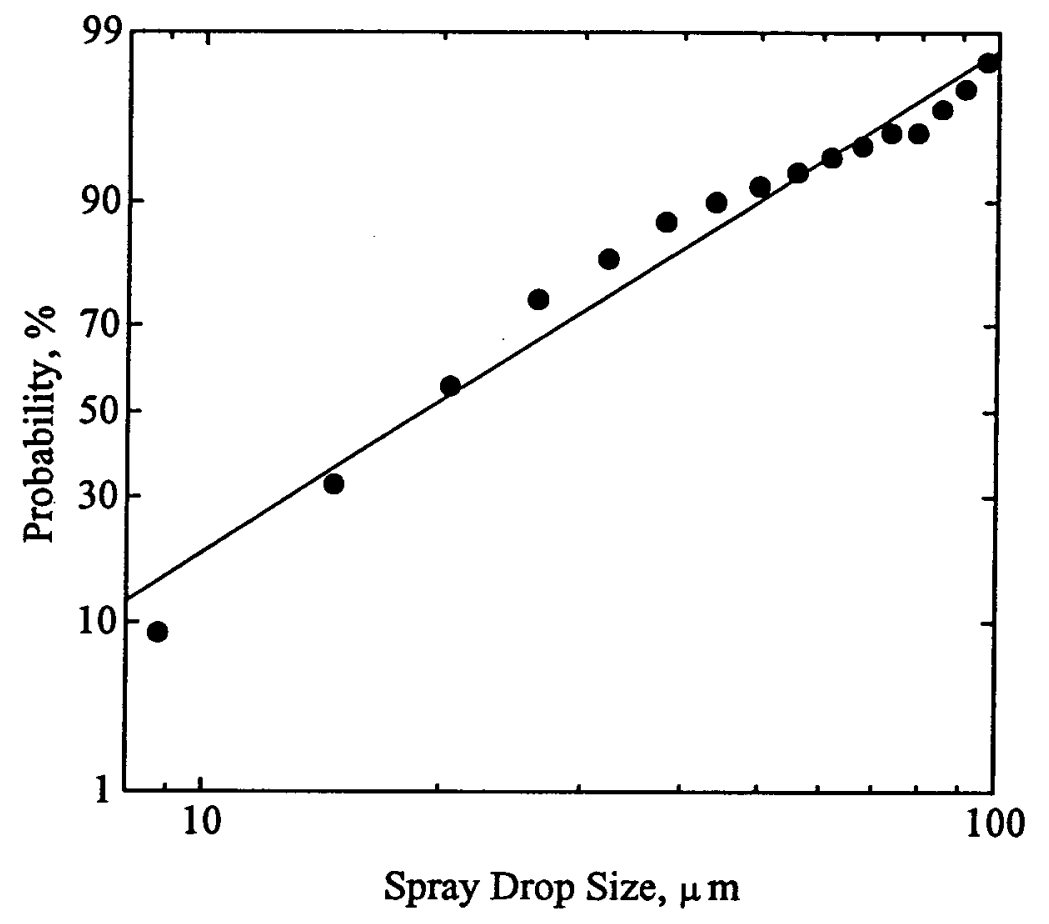

Figure 5.18: Log-normal representation of drop size distribution (Run S1a).

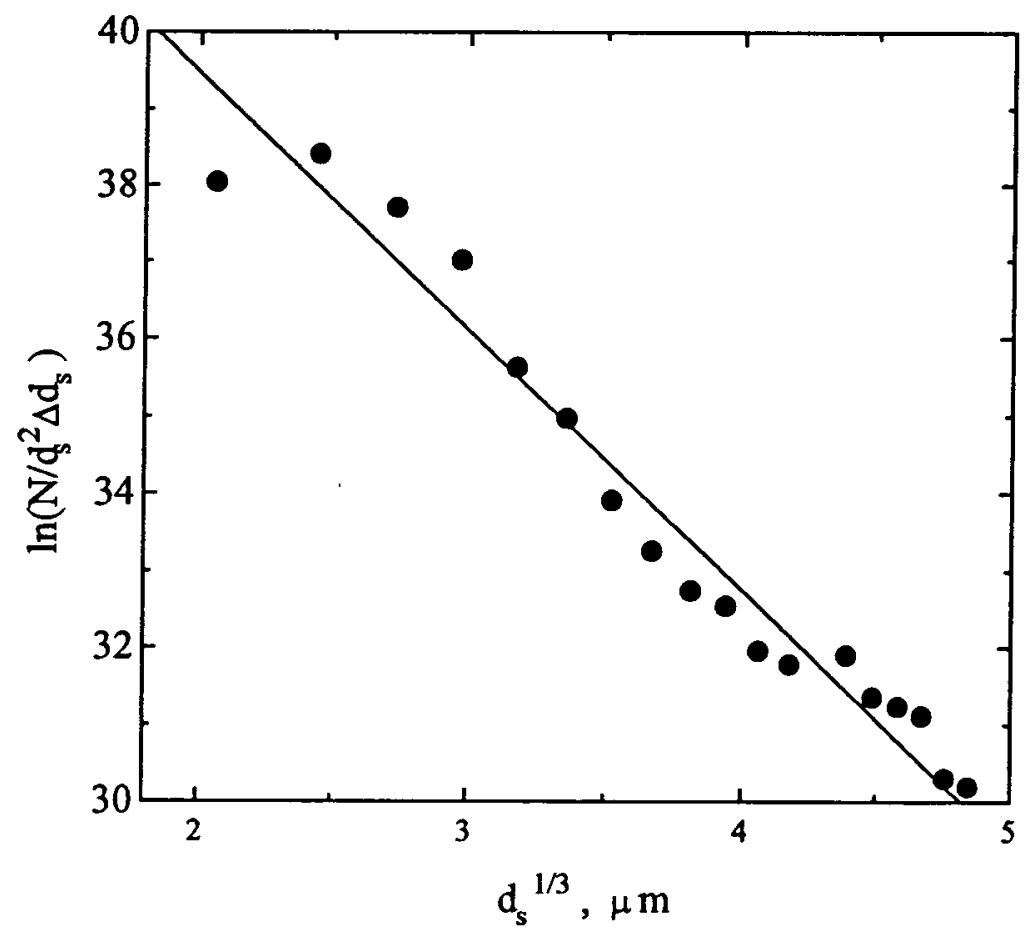

Figure 5.19: Nukiyama-Tanasawa representation of drop size distribution (index of 1/3 appeared to give optimum fit; Run S1a). 
The frequency of the droplets outside the 6 to $50 \mu \mathrm{m}$ size range was much lower (see Figure 5.17) and therefore only the droplets in the size range 6 to $50 \mu \mathrm{m}$ were used in the calculations. Two methods were employed to determine the drop size distribution: the first method employed Equations (5.11) and (5.12) to determine the coefficients for Equation (5.10); the second method used an optimization procedure to determine the coefficients directly, i.e.,

$$
\text { Minimize } \sum\left(N_{\text {counted }}-\int_{\text {cut }} \frac{\Sigma N}{\ln \sigma_{g} \sqrt{2 \pi}} \exp \left(\frac{-\left(\ln d_{s}-\ln d_{g c}\right)^{2}}{2 \ln ^{2} \sigma_{g}}\right) \mathrm{d}\left(\ln d_{s}\right)\right)^{2}
$$

where 'cut' represents the droplets in a given size range and $\Sigma N$ denotes the frequency of droplets in each cut. The values of $\sigma_{g}, d_{s}$ and $\Sigma N$ that best represent the 6 to $50 \mu \mathrm{m}$ data according to Equation (5.10) were determined using this least squares technique. Both results can be seen in Figure 5.17. Although both methods appear to give good representation to this set of data (Run S1a), the second method resulted in large errors for runs with cuts that deviated from the log-normal shape. To avoid this error, only the first method was used for all subsequent calculations including the results given in Table 5.7.

The Sauter mean diameter $\left(d_{s v}\right)$, also known as the surface-volume diameter, was then calculated from

$$
\ln d_{s v}=\ln d_{g c}+2.5 \ln ^{2} \sigma_{g}
$$

using the calculated values of $d_{g c}$ and $\sigma_{g}$. The results are presented in Tables 5.7 and 5.8. The Sauter diameter is commonly used for mass transfer operations (Mugele and Evans, 1951 ) including coating operations. For liquids atomized in small converging nozzles with compressed air, Nukiyama and Tanasawa (1939) correlated $d_{s v}$ in the following way:

$$
d_{s v}=k_{1} / u_{r}+k_{2}\left(1000 Q_{l} / Q_{a}\right)^{15}
$$

where $d_{s v}$ and $u_{r}\left(=u_{a}-u_{l}\right)$ are expressed in $\mu \mathrm{m}$ and $\mathrm{m} / \mathrm{s}$, respectively. The optimum values of the constants $k_{1}$ and $k_{2}$ were -560 and 3.45 , respectively, using the data given in Table 5.7. The drop size data obtained above the minimum pulsating velocity did not ap- 
Table 5.7: Results of drop size studies at various operating conditions.

\begin{tabular}{|c|c|c|c|c|c|c|}
\hline Run \# & $N$ & $d_{g c,} \mu \mathrm{m}$ & $\sigma_{g,} \mu \mathrm{m}$ & $d_{s v} \mu \mathrm{m}$ & $Q_{a,} \mathrm{~L} / \mathrm{s}$ & $W_{s,} \mathrm{~g} / \mathrm{s}$ \\
\hline S1a & 254 & 20.5 & 1.47 & 29.8 & 0.357 & 3.12 \\
\hline S1b & 101 & 20.3 & 1.40 & 27.0 & 0.357 & 3.12 \\
\hline S1c & 123 & 18.4 & 1.61 & 32.5 & 0.357 & 3.12 \\
\hline S1abc & 478 & 19.9 & 1.50 & 30.1 & 0.357 & 3.12 \\
\hline S2a & 223 & 16.8 & 1.54 & 26.8 & 0.314 & 3.12 \\
\hline S2b & 140 & 18.8 & 1.54 & 30.0 & 0.314 & 3.12 \\
\hline S2ab & 363 & 17.5 & 1.55 & 28.2 & 0.314 & 3.12 \\
\hline S3 & 251 & 15.8 & 1.55 & 25.4 & 0.442 & 3.12 \\
\hline S4 & 229 & 17.9 & 1.80 & 42.6 & 0.336 & 3.12 \\
\hline S5 & 87 & 15.0 & 1.56 & 24.7 & 0.314 & 2.14 \\
\hline S6 & 73 & 13.7 & 1.57 & 22.7 & 0.272 & 2.14 \\
\hline S7 & 58 & 15.7 & 1.53 & 24.2 & 0.229 & 2.14 \\
\hline S8 & 287 & 27.0 & 1.57 & 44.7 & 0.698 & 6.06 \\
\hline S9 & 184 & 16.9 & 1.88 & 45.9 & 0.570 & 6.06 \\
\hline S10 & 111 & 14.4 & 1.75 & 31.7 & 0.442 & 6.06 \\
\hline S11 & 356 & 20.3 & 1.68 & 44.3 & 0.527 & 5.08 \\
\hline S12 & 184 & 17.9 & 1.83 & 44.5 & 0.442 & 5.08 \\
\hline S13 & 430 & 18.1 & 1.76 & 40.3 & 0.612 & 5.08 \\
\hline S14 & 236 & 14.6 & 1.66 & 27.7 & 0.442 & 4.10 \\
\hline S14a & 696 & 13.5 & 1.58 & 22.8 & 0.442 & 4.10 \\
\hline S14b & 80 & 18.6 & 1.67 & 36.3 & 0.442 & 4.10 \\
\hline S14c & 744 & 14.3 & 1.65 & 26.9 & 0.442 & 4.10 \\
\hline S15 & 140 & 16.4 & 1.62 & 29.4 & 0.527 & 4.10 \\
\hline S16 & 128 & 17.2 & 1.70 & 34.4 & 0.357 & 4.10 \\
\hline
\end{tabular}

pear to follow any clear trend (see Table 5.8) and were therefore not used in the optimization calculations. The results are displayed in Figure 5.20 and show poor agreement with the measured data. The following power-law equation, determined by a least squares method, gives a better representation of the data:

$$
d_{s v}=261\left(Q_{a} / Q_{m a}\right)^{1.7}\left(Q_{l} / Q_{a}\right)^{19}
$$

The units of $d_{s v}$ are mm. This equation suggests that $Q_{a}$ and $Q_{l}$ should not be grouped together without adding a separate term to correct for their differing effects on $d_{s v}$. Equation (5.17) clearly shows (from the exponents of the $Q_{l}$ and $Q_{a}$ which are 1.9 and 0.2 , respectively) that the effect of $Q_{l}$ on $d_{s v}$ is much greater than that of $Q_{a}$. 
Table 5.8: Sauter mean diameters relative to the operating limits.

\begin{tabular}{|c|c|c|c|c|}
\hline & $\begin{array}{l}\text { Near middle of } \\
\text { operating limits }\end{array}$ & $\begin{array}{c}\text { Near pulsating } \\
\text { point }\end{array}$ & $\begin{array}{l}\text { Above pulsat- } \\
\text { ing point }\end{array}$ & $W_{s}, \mathrm{~g} / \mathrm{s}$ \\
\hline$Q_{\infty} \mathrm{L} / \mathrm{s}$ & 0.229 & 0.272 & 0.315 & \multirow[t]{2}{*}{2.14} \\
\hline$d_{\mathrm{nn}} \mu \mathrm{m}$ & 24.7 & 22.7 & 24.7 & \\
\hline$Q_{m} \mathrm{~L} / \mathrm{s}$ & 0.315 & 0.357 & 0.442 & \multirow[t]{2}{*}{3.12} \\
\hline$d_{\mathrm{nn}} \mu \mathrm{m}$ & 30.0 & 29.8 & 25.4 & \\
\hline$Q_{\infty} \mathrm{L} / \mathrm{s}$ & 0.360 & 0.442 & 0.527 & \multirow[t]{2}{*}{4.10} \\
\hline$d_{\mathrm{snn}} \mu \mathrm{m}$ & 34.4 & 27.7 & 29.4 & \\
\hline$Q_{\infty} \mathrm{L} / \mathrm{s}$ & 0.442 & 0.527 & 0.612 & \multirow[t]{2}{*}{5.08} \\
\hline$d_{s p} \mu \mathrm{m}$ & 44.5 & 44.3 & 40.3 & \\
\hline$Q_{\infty} \mathrm{L} / \mathrm{s}$ & 0.442 & 0.57 & - & \multirow[t]{2}{*}{6.06} \\
\hline$d_{s, \mu m}$ & 45.9 & 44.7 & - & \\
\hline
\end{tabular}

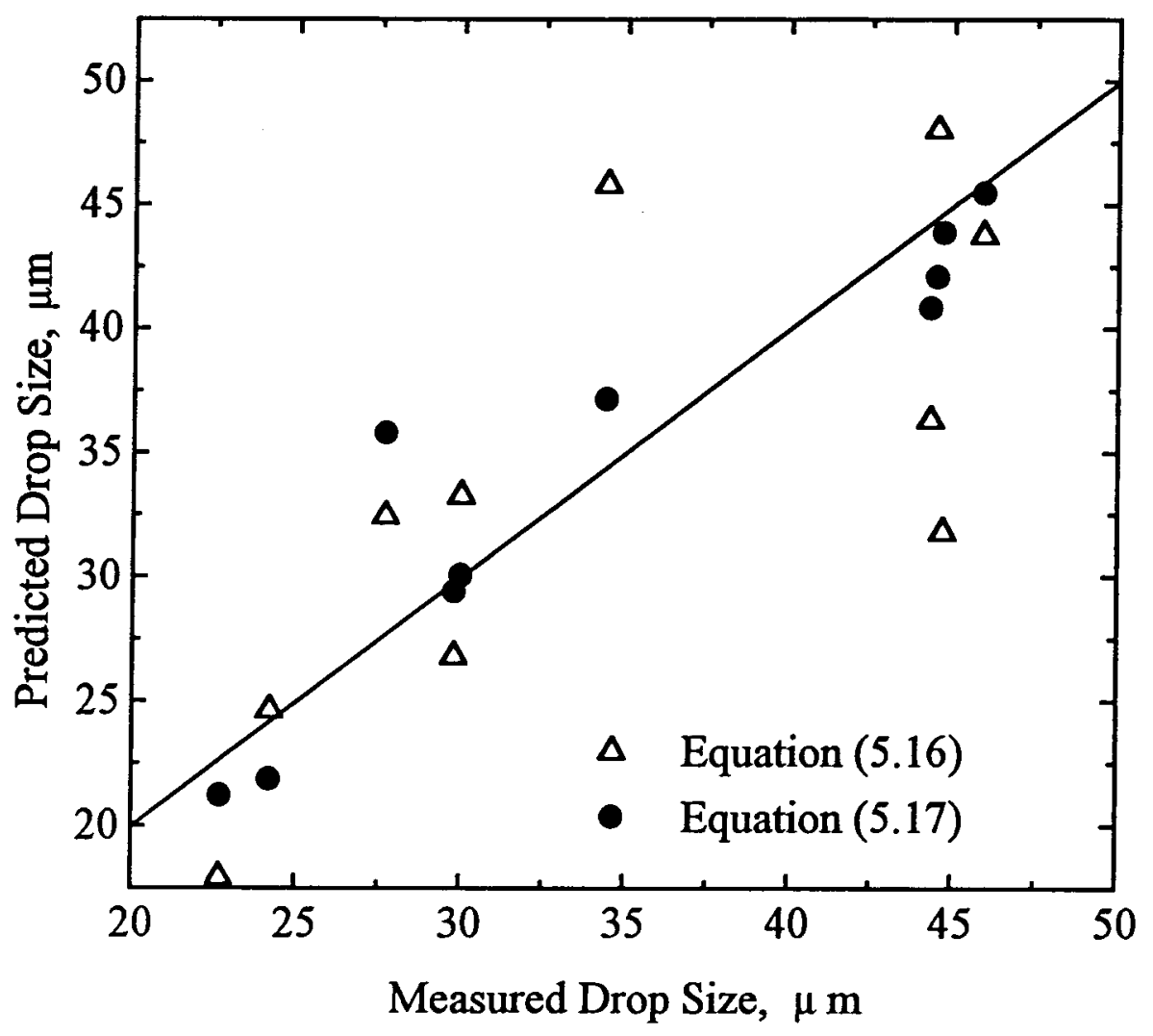

Figure 5.20: Predictions of Sauter mean diameter. 
The main errors in $d_{s v}$ were suspected to arise from uncertainties associated with the sample size, sample location, and sampling procedure. The errors associated with the sample size and the sample locations were investigated in runs S1a to $S 2 b$ (see Table 5.7), and the largest error was $\pm 10 \%$. The maximum error associated with the rotation speed of the protective tube was even higher at $\pm 31 \%$ (runs S14 and S14b).

In the model coating calculations which follow, it is assumed that all sulfur droplets have the same size equal to the Sauter mean diameter calculated from Equation (5.17).

\subsubsection{Coating Mechanism and Sulfur Spray Concentration}

The assumption that inertial deposition is the dominating mechanism for the deposition of sulfur droplets on the bed particles is verified by using the collection efficiency equations suggested by Clift et al. (1981). Table 5.9 shows typical values of the collection efficiency due to diffusion $\left(E_{D}\right)$, inertial deposition $\left(E_{I}\right)$, gravitational settling $\left(E_{G}\right)$ and direct inter-

Table 5.9: Mechanical collection efficiency (using correlations suggested by Clift et al., 1981 and Behie et al., 1972) at selected operating conditions in the spout $\left(\rho_{c}\right.$ $\left.=1773 \mathrm{~kg} / \mathrm{m}^{3}, d_{p}=2.16 \mathrm{~mm}, \mu=2.08 \times 10^{-5} \mathrm{~kg} / \mathrm{m} \cdot \mathrm{s}\right)$.

\begin{tabular}{|c|c|c|c|c|}
\hline$U, \mathrm{~m} / \mathrm{s}$ & \multicolumn{2}{|c|}{5} & \multicolumn{2}{|c|}{50} \\
\hline$d_{s, \mu \mathrm{m}}$ & 6 & 50 & 6 & 50 \\
\hline$S_{\perp}$ & 0.40 & 27 & 4.0 & 275 \\
\hline$E_{D}=(4.36 / \varepsilon)\left(\wp / U d_{s}\right)^{2 / 3}$ & $3.3 \times 10^{-6}$ & $7.9 \times 10^{-7}$ & $7.1 \times 10^{-7}$ & $1.7 \times 10^{-7}$ \\
\hline $\begin{aligned} E_{I}= & 0.0036-0.2323 S_{t}+2.422 S_{t}^{2} \\
& -2.033 S_{t}^{3}, 0.083<S_{t} \leq 0.6\end{aligned}$ & 0.165 & - & - & - \\
\hline$E_{I}=S_{t}^{2} /\left(S_{t}+0.5\right)^{2}, S_{t}>0.6$ & - & 0.965 & 0.788 & 0.996 \\
\hline$E_{G}=0.075 \sqrt{N_{G}}$ & 0.00074 & 0.0061 & 0.00023 & 0.0019 \\
\hline$E_{D I}=6.3 N_{D I}^{2} \varepsilon^{-2.4}$ & 0.00011 & 0.0077 & 0.00011 & 0.0077 \\
\hline
\end{tabular}


ception $\left(E_{D l}\right)$ at selected operating conditions. The values of the air velocity $(U)$ and the droplet size $\left(d_{s}\right)$ given in Table 5.9 are close to the operating conditions in the spout. The results clearly indicate that inertial deposition is the dominating collection mechanism under the present coating conditions.

The collection efficiency alone, however, is not sufficient to calculate the sulfur droplet deposition rate onto bed particles; information on the spray distribution above the nozzle is required also. Consequently, experiments were attempted to determine the spray distribution; however, a suitable device for detecting the sulfur concentration in the bed could not be developed. A sampler without a heated tip could not deal with the molten sulfur spray, which plugged up the tip of the sampler. Consequently, efforts were made to heat the tip electrically (to oxidize the sulfur) with $\mathrm{NiCr}$ wires wrapped around the tip. Unfortunately, the wires only lasted a few seconds in the bed because they could not withstand collisions with the fast moving bed particles and the presence of urea dust which left carbon residue on wires. Internal heating of the tip was not possible due to space limitations of the sampler (i.e., the sampler had to be small enough to be placed in the spout without significantly upsetting the upstream flow). The details of the samplers which were tested in this work are provided in Appendix I.

Since the spray distribution could not be measured directly, the assumptions concerning the spray angle (see Chapter 4$)$ and the height $\left(h_{f d s}\right)$ at which the sulfur droplets start to deposit onto the bed particles could not be verified. However, the angle and height could be deduced from the particle coating distribution, which is the topic of the next section. The predicted sulfur concentration profiles for various spray angles for Run $\mathrm{C} 17$ are shown in Figure 5.21; corresponding values for various coating runs using a spray angle of $20^{\circ}$ are shown in Figure 5.22. The operating conditions for the runs shown in Figures 5.21 and 5.22 are given in Table 5.11. Decreasing spout velocity causes the initial increase in the sulfur concentration until $z=h_{f d s}$. Note that in Figure 5.21, the spray angle 


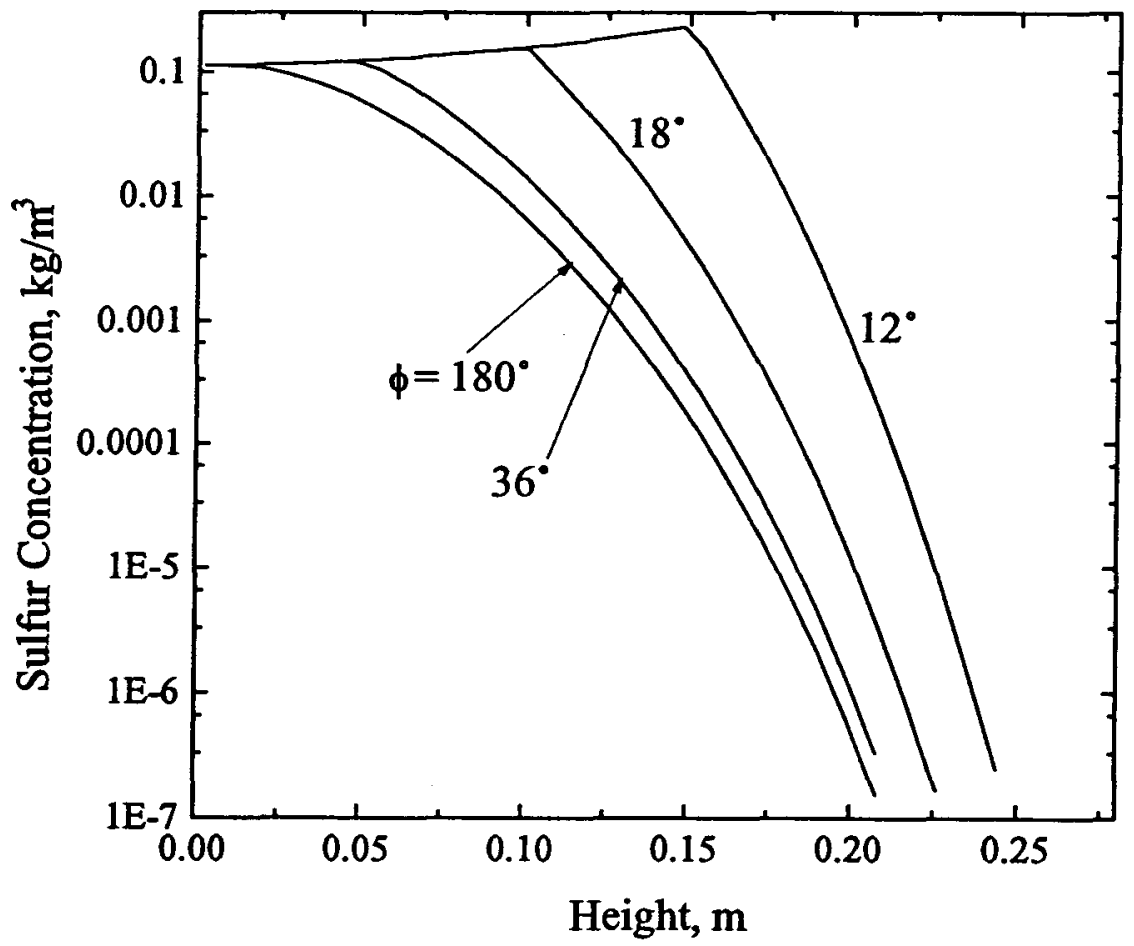

Figure 5.21: Predicted sulfur concentration profile for various spray angles.

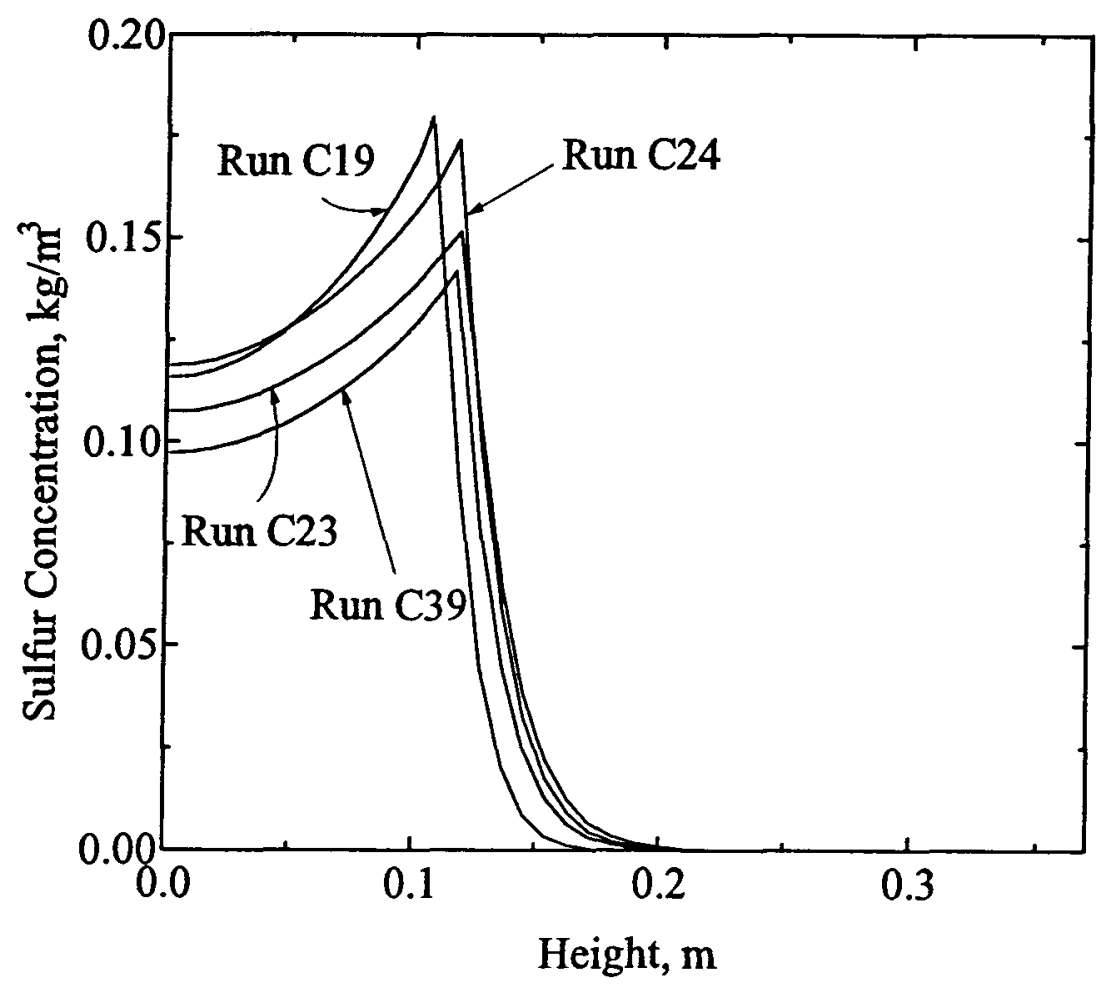

Figure 5.22: Predicted sulfur concentration profile for various coating runs $\left(\phi=20^{\circ}\right)$. 
of $180^{\circ}$ corresponds to completely dispersed spray droplets in the main air stream or $h_{f d s}=$ 0 ; in reality, such a spray angle does not exist.

\subsection{Coating Distribution and Product Quality}

The coating distribution and quality of products were measured according to the methods outlined in Chapter 3. The coating distribution was also calculated using the models presented in Chapter 4 and the product quality was calculated using the methods outlined in this section. The operating ranges applicable to this study are listed in Table 5.10, and the experimental and model results are given in Table 5.11.

One deviation from the methods described in Chapter 3 relates to the measurement of the urea feed rate. The urea feed rates often did not match the calibrated rates (see Appendix IV); as a result, the urea feed rate $\left(W_{u}\right)$ was determined from the product discharge rate $\left(W_{p}\right)$ and the mean sulfur content found by the crush test $\left(\bar{X}_{s m}\right)$ : i.e.,

$$
W_{u}=W_{p}\left(1-\bar{X}_{s m}\right)
$$

The mean sulfur content $\left(\bar{X}_{s}\right)$ based on the feed rates of urea and sulfur (also given in Table 5.11) was never in complete agreement with and was usually somewhat larger than $\bar{X}_{s m}$. One reason for the discrepancy is that the products may have been sampled before steady state conditions were achieved. Consequently, the predicted product quality, $U_{D}$, (see Section 5.3.2) given in Table 5.10 is based on $\bar{X}_{s}$ rather than $\bar{X}_{s m}$ and represents the seven day urea dissolution value for the products obtained under steady state operations.

It should be noted that comparisons between the coating distribution predictions of Models II and III with Models I and IV are not possible as the relationships between the variables in Models II and III to the operating variables were not determined in this work (see Chapter 4). Furthermore, the predictions of Models II and III could not be compared directly with the measured results for the same reason. Consequently, the discussions regar- 
Table 5.10: Operating range applicable to coating distribution and product quality studies.

\begin{tabular}{|l|c|}
\hline Operating Variable & Operating Range \\
\hline Spouting air flow rate, $Q_{s}$ & $20.1-29.2 \mathrm{~L} / \mathrm{s}$ \\
\hline Atomizing air flow rate, $Q_{a}$ & $0.23-0.78 \mathrm{~L} / \mathrm{s}$ \\
\hline Bed Height, $H$ & $0.28-0.36 \mathrm{~m}$ \\
\hline Bed Diameter, $D$ & 0.24 and $0.45 \mathrm{~m}$ \\
\hline Spouting air orifice diameter, $d_{i}$ & $24.7-35.1 \mathrm{~mm}$ \\
\hline Urea feed rate, $W_{\nu}$ & $7.6-19.9 \mathrm{~g} / \mathrm{s}$ \\
\hline Sulfur injection rate, $W_{s}$ & $3.12-6.06 \mathrm{~g} / \mathrm{s}$ \\
\hline Bed Temperature, $T_{b}$ & $57-64{ }^{\circ} \mathrm{C}$ \\
\hline
\end{tabular}

Table 5.11: Operating conditions investigated for the coating study.

\begin{tabular}{|c|c|c|c|c|c|c|c|c|c|c|c|c|}
\hline $\begin{array}{c}\text { Run } \\
\#\end{array}$ & $\begin{array}{c}D_{i} \\
(\mathrm{~mm})\end{array}$ & $\begin{array}{c}Q_{s} \\
(\mathrm{~L} / \mathrm{s})\end{array}$ & $\begin{array}{c}Q_{a} \\
(\mathrm{~L} / \mathrm{s})\end{array}$ & $\begin{array}{c}W_{s} \\
(\mathrm{~g} / \mathrm{s})\end{array}$ & $\begin{array}{c}W_{u} \\
(\mathrm{~g} / \mathrm{s})\end{array}$ & $\begin{array}{c}T_{b} \\
\left({ }^{\circ} \mathrm{C}\right)\end{array}$ & $\begin{array}{c}D \\
(\mathrm{~m})\end{array}$ & $\begin{array}{c}H \\
(\mathrm{~m})\end{array}$ & $\begin{array}{c}\bar{X}_{s m} \\
\%\end{array}$ & $\begin{array}{c}U_{D m} \\
\%\end{array}$ & $\begin{array}{c}\bar{X}_{s} \\
\%\end{array}$ & $\begin{array}{c}U_{D} \\
\%\end{array}$ \\
\hline $\mathrm{C} 17$ & 24.7 & 21.1 & 0.44 & 4.1 & 8.6 & 57 & 0.24 & 0.28 & 25.9 & 50.7 & 32.3 & 42.2 \\
\hline C19 & 24.7 & 21.1 & 0.44 & 4.1 & 5.65 & 57 & 0.24 & 0.28 & 32.6 & 37.5 & 42.1 & 32.8 \\
\hline C20 & 24.7 & 21.1 & 0.44 & 4.1 & 14.6 & 57 & 0.24 & 0.28 & 23.0 & 64.2 & 21.9 & 59.4 \\
\hline C21 & 24.7 & 21.1 & 0.44 & 4.1 & 19.9 & 57 & 0.24 & 0.28 & 17.1 & 69.2 & 17.1 & 70.0 \\
\hline C22 & 24.7 & 22.8 & 0.23 & 5.08 & 10.1 & 63 & 0.24 & 0.28 & 30.0 & 52.3 & 33.3 & 41.9 \\
\hline C23 & 24.7 & 24.5 & 0.44 & 5.08 & 8.75 & 64 & 0.24 & 0.28 & 32.7 & 44.1 & 36.8 & 38.5 \\
\hline C24 & 24.7 & 22.8 & 0.57 & 5.08 & 10.1 & 64 & 0.24 & 0.28 & 28.0 & 40.6 & 33.4 & 41.6 \\
\hline C25 & 24.7 & 20.1 & 0.44 & 4.1 & 9.91 & 64 & 0.24 & 0.28 & 26.1 & 48.2 & 29.3 & 46.8 \\
\hline C26 & 24.7 & 22.8 & 0.44 & 4.1 & 9.73 & 64 & 0.24 & 0.28 & 23.1 & 46.3 & 29.7 & 46.6 \\
\hline C29 & 28.2 & 24.5 & 0.44 & 4.1 & 9.92 & 64 & 0.24 & 0.28 & 22.5 & 51.0 & 29.3 & 47.7 \\
\hline C30 & 35.1 & 29.2 & 0.44 & 4.1 & 10.5 & 64 & 0.24 & 0.28 & 21.8 & 74.6 & 28.2 & $\mathrm{n} / \mathrm{s}$ \\
\hline C31 & 24.7 & 21.1 & 0.23 & 3.12 & 9.43 & 60 & 0.24 & 0.28 & 21.1 & 64.7 & 24.9 & 54.0 \\
\hline C33 & 24.7 & 21.1 & 0.53 & 6.06 & 11.3 & 60 & 0.24 & 0.28 & 24.5 & 38.0 & 34.9 & 39.2 \\
\hline C38 & 24.7 & 22.8 & 0.44 & 4.1 & 11.7 & 60 & 0.24 & 0.36 & 23.2 & 51.5 & 25.9 & 52.3 \\
\hline C39 & 24.7 & 27.6 & 0.44 & 4.1 & 7.6 & 64 & 0.45 & 0.355 & 17.6 & 49.9 & 35.1 & $\mathrm{n} / \mathrm{s}$ \\
\hline C40 & 24.7 & 27.6 & 0.78 & 6.06 & 11.1 & 64 & 0.45 & 0.355 & 15.3 & 72.4 & 35.3 & $\mathrm{n} / \mathrm{s}$ \\
\hline
\end{tabular}


ding Models II and III results are mostly confined to a sensitivity analysis of the model variables.

\subsubsection{Coating Distribution}

Forty sulfur coated urea particles were randomly sampled and the sulfur content of the individual particles were determined using the particle crush test described in Chapter 3. The sulfur content $\left(X_{s p, i}\right)$ was then normalized according to

$$
\left(X_{s p, i}\right)_{n e w}=X_{s p, i} \bar{X}_{s p} / \bar{X}_{s m}
$$

where $\bar{X}_{s p}$ is the average sulfur content of the 40 particles sampled. The results can be seen in Figures 5.23 and 5.24 for Runs C17 and C38, respectively; the results for other selected runs are provided in Appendix II.

As shown by Figure 5.24, Model IV with the spray angle $(\phi)$ of $20^{\circ}$ and Model I appear to give good predictions of the measured sulfur content distributions. Both models, however, under-predicted the amount of the inadequately coated particles (i.e., $X_{s}<10 \%$ ). This result was expected for Model I as discussed already in Chapter 4. In case of Model IV, the size of the actual spray zone was suspected to be lower than that predicted, which meant that the actual spray angle was larger or that coating occurred below the assumed $h_{f d s}$. It is unlikely that the spray angle is larger than the angle provided by the manufacturer $\left(\phi=20^{\circ}\right)$ as the angle should decrease in the presence of spouting air (see Equation (5.5)). Therefore, $h_{f d s}$ was suspected to be incorrect.

Consequently, the effect of $h_{f d s}$ was investigated by examining the coating distribution of batch products. The batch products were used because the effects of the feed location and exit probability are absent, and the influence of $h_{f d s}$ can be isolated. Figure 5.25 shows the coating distributions for the batch products coated for 210 and $480 \mathrm{~s}$ under the following conditions: $W_{s} \approx 3.5 \mathrm{~g} / \mathrm{s}, H \approx 0.3 \mathrm{~m}, D=0.24 \mathrm{~m}, T \approx 60^{\circ} \mathrm{C}$ and $d_{i}=28.2 \mathrm{~mm}$. 


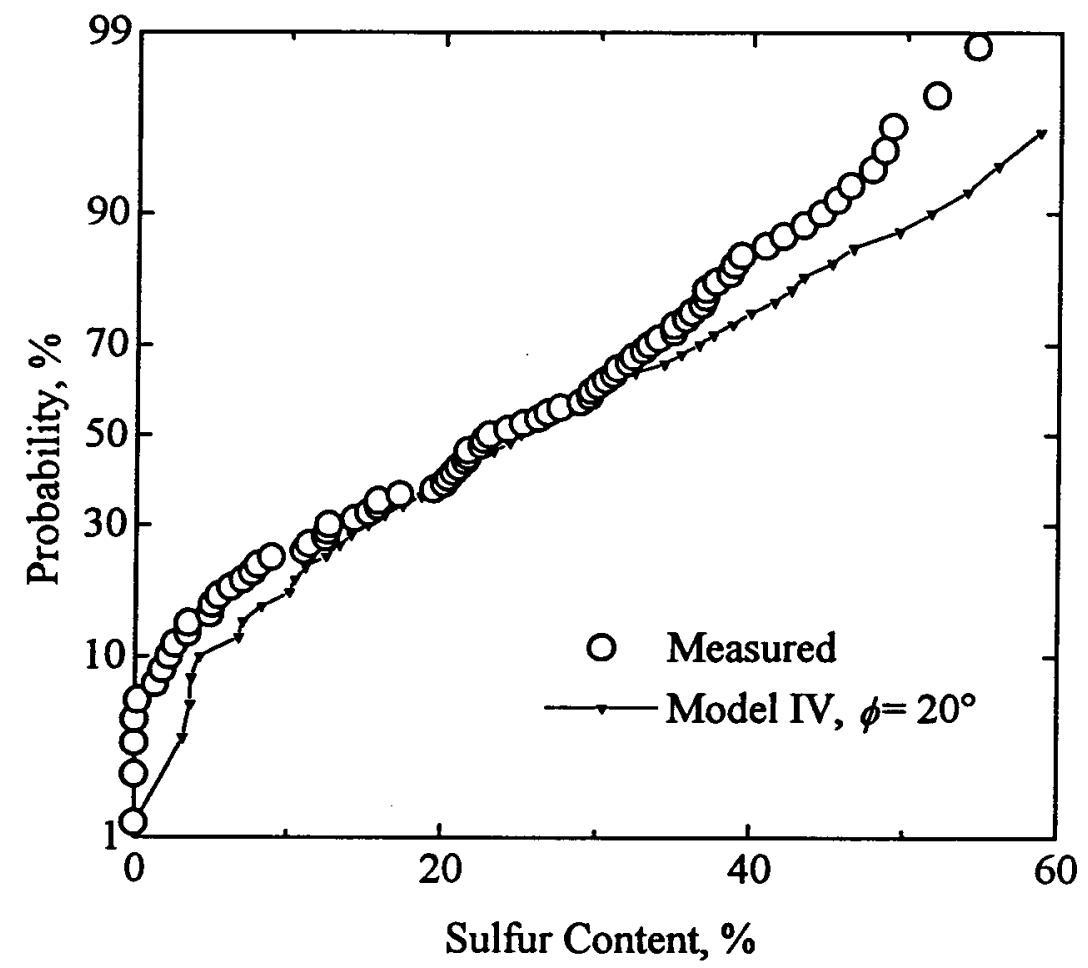

Figure 5.23: Comparison between measured and predicted coating distributions for Run C17.

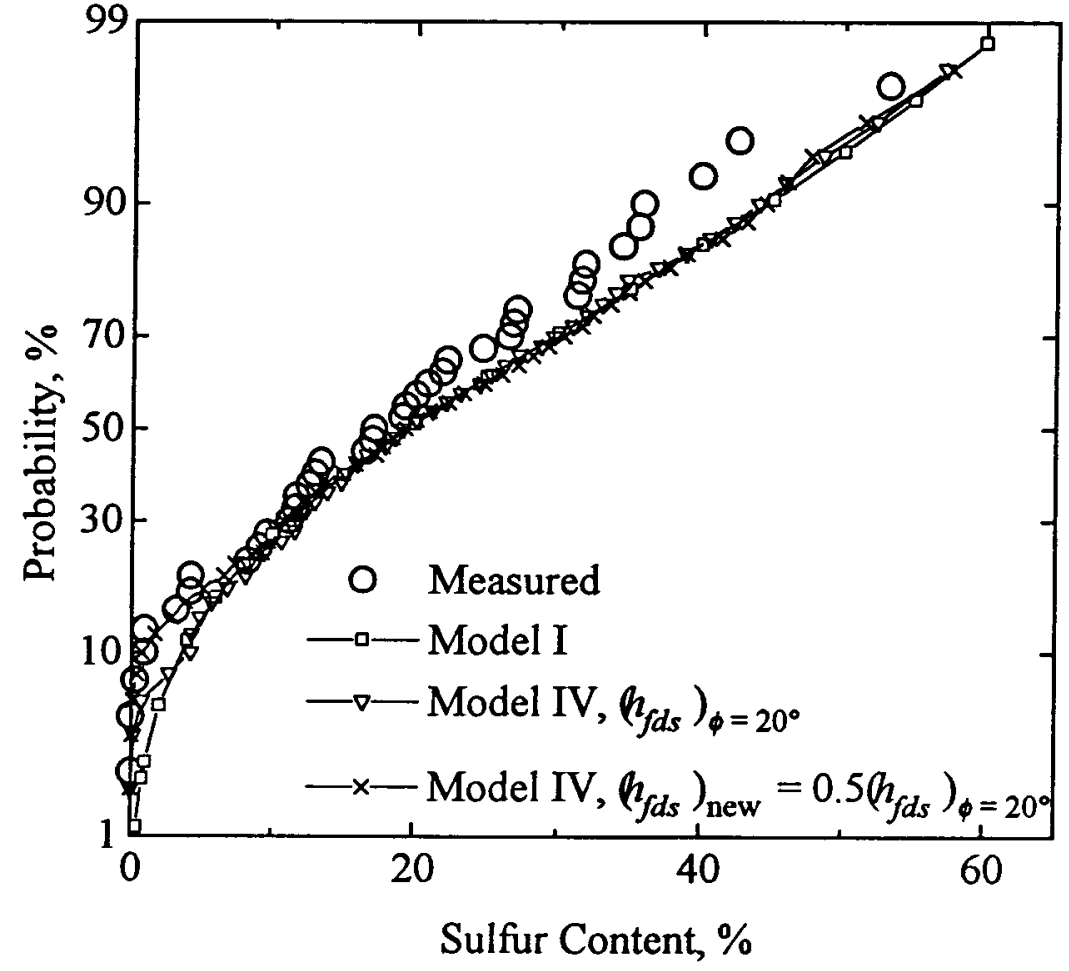

Figure 5.24: Comparison between measured and predicted coating distributions for Run C38. 


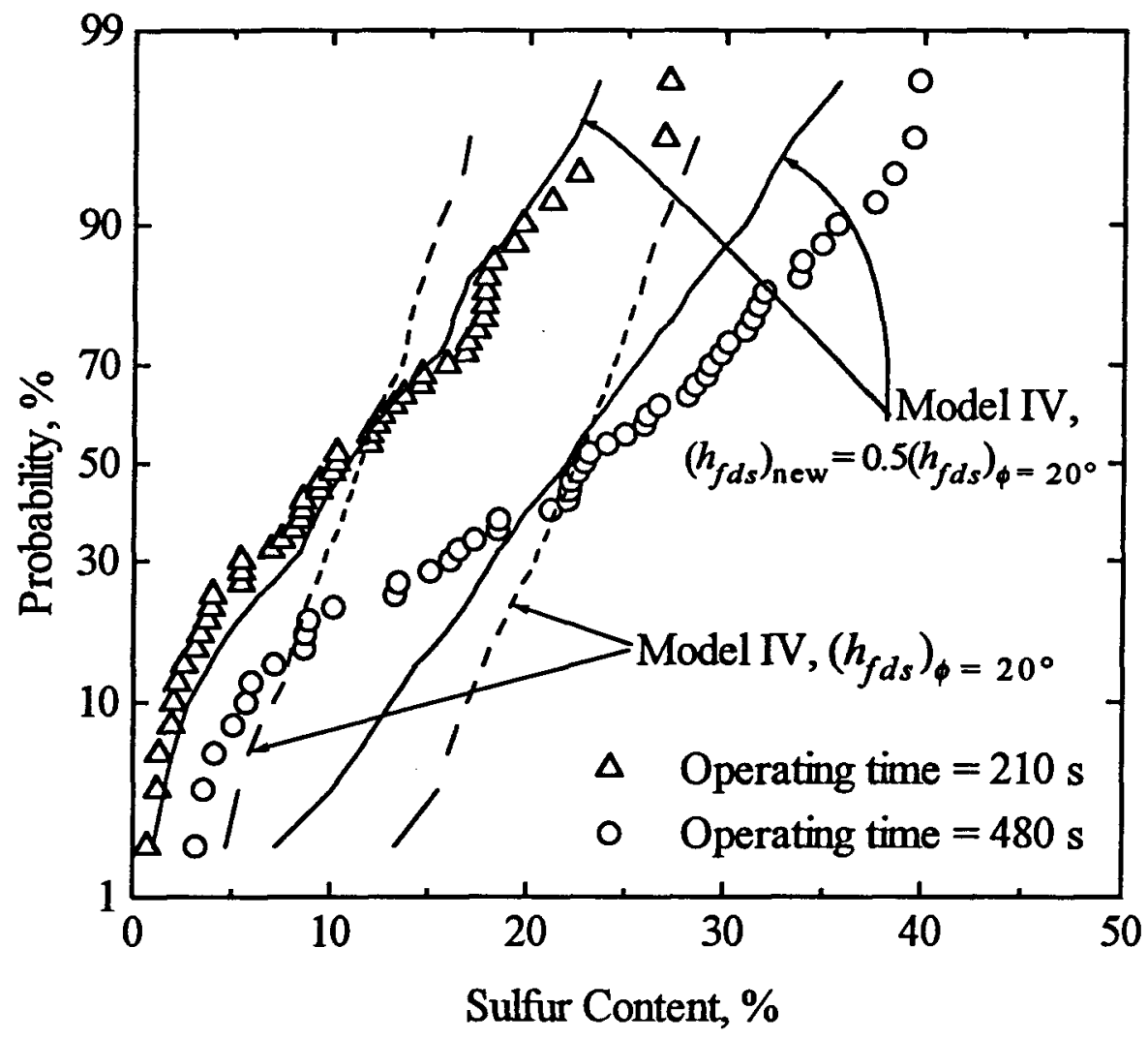

Figure 5.25: Coating distributions of batch products.

Again, the model under-predicted the amount of inadequately coated particles but, when $\left(h_{f d s}\right)_{\text {new }}=0.5\left(h_{f d s}\right)_{\phi=20^{\circ}}$ was used, the predictions improved considerably. The physical explanation for this result (assuming the spray angle is fixed at $20^{\circ}$ ) is that the bed particles start to be coated at a height which is approximately half of the distance at which the spray reaches the spout-annulus interface. Significant improvements were also noted for the continuous data in both Figure 5.24 when these new $h_{f d s}$ values were used in the calculations.

The discrepancy between the measured and predicted distributions may be attributed to uncertainties in the measurements of the sulfur flow rate, urea feed rate, and individual particle sulfur content. The error in the sulfur flow rate is typically \pm 1 scale reading on the sulfur rotameter which amounts to less than $\pm 5 \%$ error in the flow rate. The error in 
the urea feed rate using Equation (5.18) is less than $\pm 9 \%$. The largest error contributing to the coating distribution is probably the sulfur content measurements of individual particles (see Chapter 3); the error was as large as $\pm 25 \%$ in determining the urea content of individual particles. The errors in the measurements of $\bar{X}_{s m}$ and $U_{D m}$ amounted to less than $3 \%$.

The discrepancies between the measured and predicted distributions shown in Figures 5.23 to 5.25 fall within the maximum measurement errors; however, consistently lower values of $\bar{X}_{s m}$ compared to $\bar{X}_{s}$ in Table 5.11 suggest that other errors may be involved. Other errors were suspected to result from the small sample sizes used to measure the coating distribution and from sampling beds operating under unsteady state conditions. These matters are further investigated in Section 5.3.3.

\subsubsection{Product Quality}

The measured and predicted values of the product quality (expressed in terms of the seven day dissolution test) are given in Table 5.11. In determining the predicted values, the quality of an individual particle was assumed to be the same as that of batch products having the same sulfur content.

The quality of the batch product was measured and correlated using a hyperbolic function which was found to give a good representation of the data: i.e.,

$$
U_{D, i}=\frac{1}{2}\left[\tanh \left(\frac{c_{1}-X_{s p, i}}{X_{s p, i}\left(c_{2}-X_{s p, i}\right)}\right)+1\right]
$$

where $U_{D, i}$ and $X_{s p, i}$ represent the seven day urea dissolution rate and sulfur content, respectively. Twenty-three batch runs were conducted to find the optimum values of $c_{1}$ and $c_{2}$ which were 0.198 and 0.715 , respectively, using a non-linear least squares optimization method. The results are plotted as Figure 5.26. All runs were conducted under the fol- 


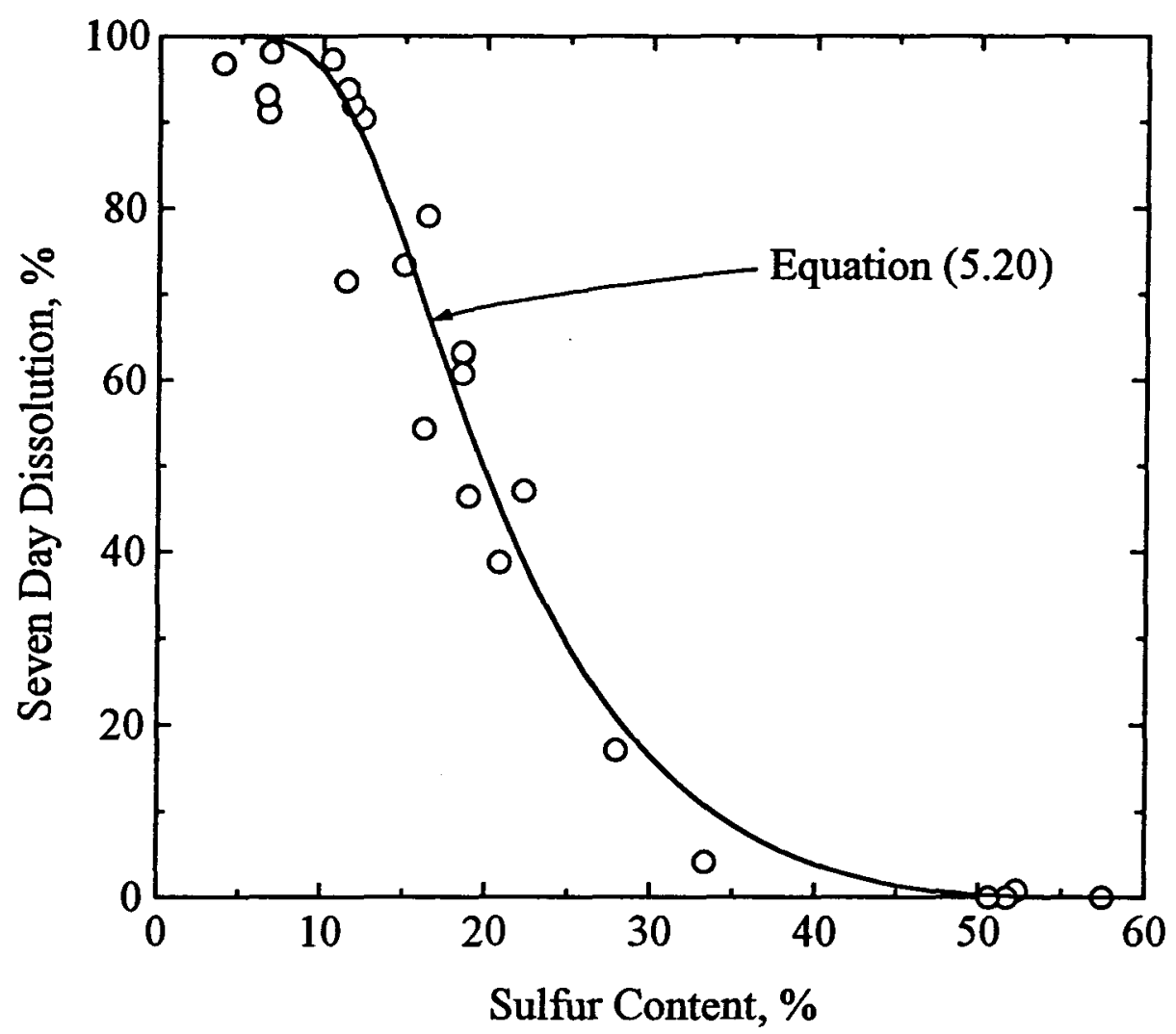

Figure 5.26: Relationship between 7-day dissolution and sulfur content for batch products.

lowing initial operating conditions: $T=60^{\circ} \mathrm{C}, H=0.25 \mathrm{~m}, D=0.24 \mathrm{~m}, d_{i}=24.7 \mathrm{~mm}, Q_{s}$ $=25 \mathrm{~L} / \mathrm{s}, Q_{a}=0.44 \mathrm{~L} / \mathrm{s}$ and $W_{s}=4.1 \mathrm{~g} / \mathrm{s}$. Adjustment in the spouting air flow rate $\left(Q_{s}\right)$ was required to keep the bed from collapsing, and the bed temperature (T) varied up to 15 ${ }^{\circ} \mathrm{C}$ due to the steady injection of molten sulfur (introduced at $\sim 150{ }^{\circ} \mathrm{C}$ ). Other than these operating variables, only the operating time was varied to control the sulfur content of the products.

The predicted dissolution values $\left(U_{D, i}\right)$ were calculated from Equation (5.20) and averaged to determine the predicted product quality $\left(U_{D}\right)$ given in Figure 5.27 and Table 5.11. All model predictions are based on steady state operations. Of all runs listed in Table 5.11, only four runs were approximately under steady state when product sampling occurred 


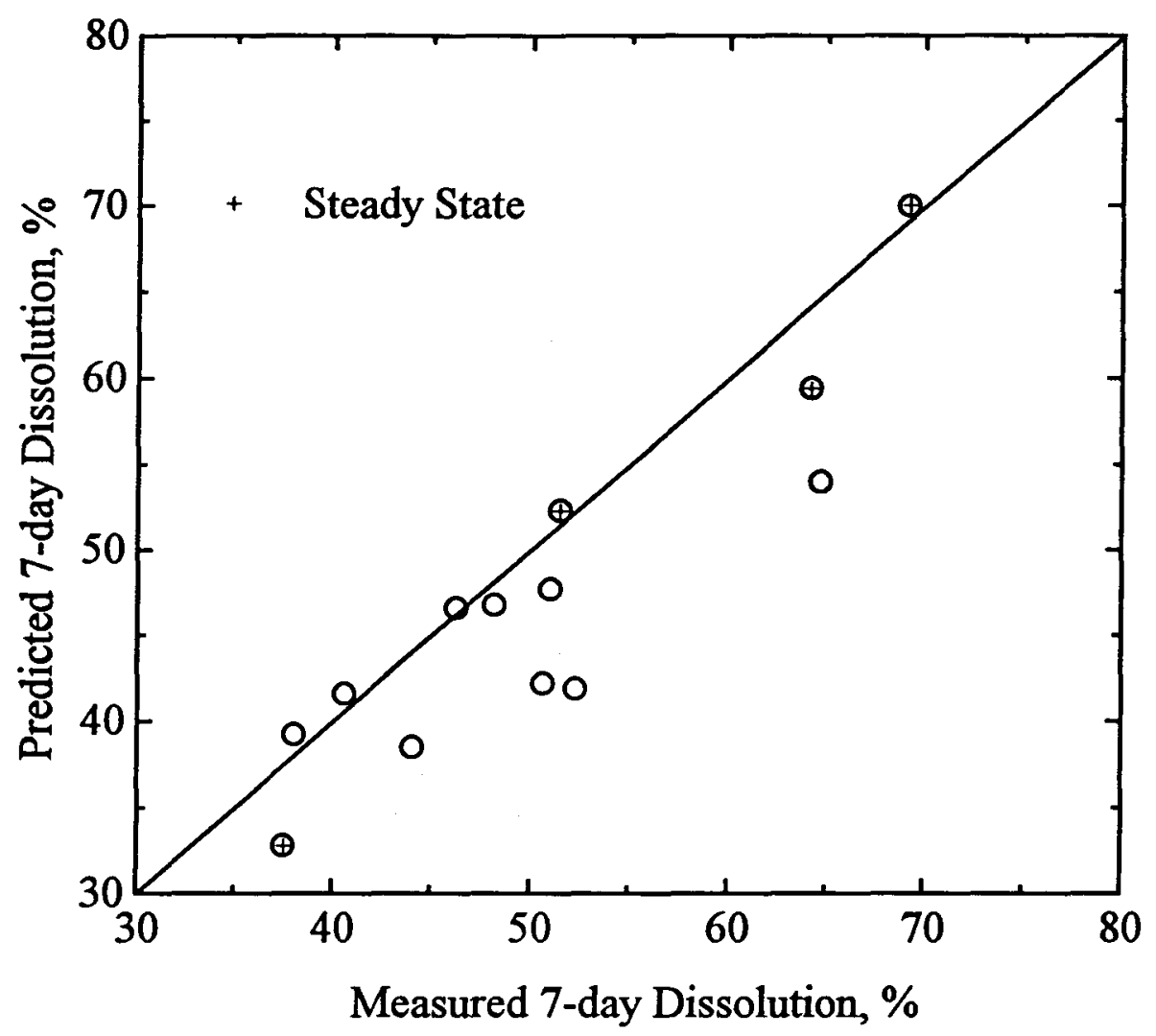

Figure 5.27: Comparison between measured and predicted product quality.

(see next section), and they are noted in Figure 5.27. Other runs had probably not reached steady state when sampling occurred and, as a result, the measured urea dissolutions were higher than the predicted dissolutions.

\subsubsection{Effect of Operating and Model Variables on Coating Distribution}

\subsubsection{Effect of Operating Time}

All product samples were collected after (at least) ten minutes of coating operation at which time steady state was assumed to exist (see Chapter 3). For a typical coating operation (e.g., $W_{p}=14 \mathrm{~g} / \mathrm{s}, \rho_{p}=1400 \mathrm{~kg} / \mathrm{m}^{3}, \varepsilon=0.42, H=0.28 \mathrm{~m}$ and $D=0.24 \mathrm{~m}$ ), the average residence time $(\bar{t})$ of the bed particles is $\sim 400 \mathrm{~s}$, and ten minutes $(t=600 \mathrm{~s})$ of operation corresponds to $t / \bar{t}=1.5$ and $F(t) \cong 0.8$ for a perfectly mixed bed of particles 
(see Equation (4.1)). $(F(t)=1$ indicates the true steady state condition, and occurs when $t$ $\rightarrow \infty$.) For most coating runs, the average coating time was approximately 20 minutes which corresponds to $t / \bar{t}=3$ and $F(t) \cong 0.95$.

The large values of $F(t)$ indicate that most of the runs were close to steady state conditions; however, the coating distribution and sulfur content results indicated that some runs might not have reached steady state at the time of sampling. Consequently, an experiment was conducted to investigate this effect. The effect of the operating time was investigated in a deep bed $(0.36 \mathrm{~m})$ with a very low urea feed rate (approximately $4 \mathrm{~g} / \mathrm{s}$ ) to accentuate the effect. The average residence time of the particles under this condition was $\sim 20 \mathrm{~min}$ utes. The results can be seen in Figure 5.28. As indicated by the changing shapes of the coating distribution curve, steady state was not achieved after ten minutes $(t / \bar{t}=0.5)$ of coating operation. Even after operating for two hours $(t / \bar{t}=6$ and $F(t)=0.998$; i.e., very close to steady state for a perfectly mixed bed), the sulfur content of the heavily coated fraction was still changing. Similar trends were also found in Tsai's (1986) investigation with a $0.15 \mathrm{~m}$ bed after three hours of continuous operation.

An explanation for this result is that the spouted bed does not behave as a perfectly mixed bed. The spouted bed may be seen as a partly well-mixed and partly plug-flow vessel in which the bed particles spend most of their time in the plug flow zone (annulus). Consequently, the spouted bed approaches steady state more slowly than does a perfectly mixed vessel.

The steady state condition is also indicated by the values of sulfur content. The measured sulfur content values $\left(\bar{X}_{s m}\right)$ for the three samples collected are given in Figure 5.28 and the sulfur content based on the feed rates of urea and sulfur $\left(\bar{X}_{s}\right)$ was $42.3 \%$. When steady state is achieved, $\bar{X}_{s m}$ should approach $\bar{X}_{s}$. (The fact that the $\bar{X}_{s m}$ values for the one and two hour runs are slightly higher than the $\bar{X}_{s}$ value is probably due to uncertainty in pro- 


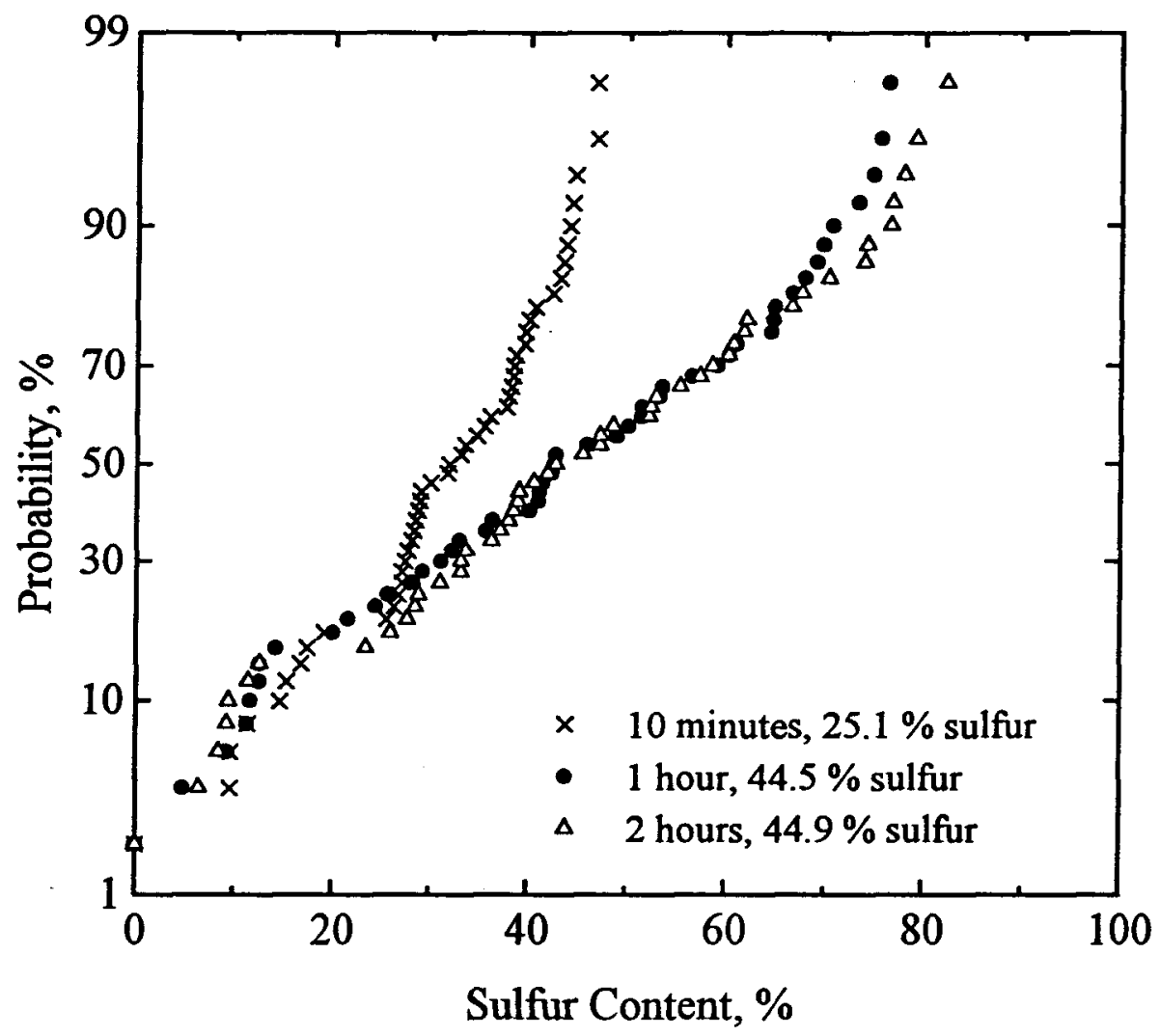

Figure 5.28: Effect of operating period on measured coating distribution.

duction rate measurement). Consequently, the sulfur content can be used as a measure to determine whether steady state was achieved. In this work, steady state was assumed to exist if the following condition was met:

$$
\bar{X}_{s}-\bar{X}_{s m} \leq 3 \%
$$

The value of $3 \%$ was chosen based on the uncertainty of the crush test (see Chapter 3). The steady state results based on Equation (5.23) are noted in Figure 5.27.

The results from this investigation provide an explanation for the discrepancy between the predicted and measured results for the heavily coated fraction in Run 17 (see Figure 5.23). The product was probably sampled from the bed while it was still under a transient operation (e.g., $\bar{X}_{s}-\bar{X}_{s m}>6 \%$; see Table 5.11 ) and, as a result, its heavily coated fraction is shown to deviate from the model steady-state predictions. 


\subsubsection{Effect of Sample Size}

Ideally, the sample size should be as large as possible to obtain a small sampling error; however, the sample size is limited by the resources available for the analysis. The sample sizes used to determine the coating distributions in this work were limited to 1000 and 40 particles for numerical (Monte Carlo) and manual sampling, respectively. These sizes were chosen on the basis of the costs associated with computer time and the time required to analyze the coated urea products.

\subsection{Numerical Sampling}

The errors associated with sample sizes and the effect of sample size on coating distribution were investigated for the various mathematical models. The errors associated with the sample sizes were determined by comparing the results of Models II and III. Models II and III were chosen for this purpose because both models should yield identical results when $a=0$ for Model III (see Equation (4.19)), but the methods of solution are different - i.e., the Model II solutions are exact whereas those of Model III are based on the numerically approximate Monte Carlo method. The differences between the model predictions of the average coating amount were calculated from

$$
\sigma=\sqrt{\sum_{i=1}^{n}\left[\left(m_{s 1}\right)_{i}-\left(m_{s 1}\right)_{\infty}\right]^{2} / n_{s i m}},
$$

where $\left(m_{s 1}\right)_{i}$ represents the Model III results given by Equation (4.22), $\left(m_{s 1}\right)_{\infty}$ represents the Model II results given by Equation (4.11), and $n_{\text {sim }}$ represents the number of simulations performed. The results are summarized in Table 5.12 and include three simulations per sample size. For each simulation, a different seed or initialization was used to generate random numbers. Note that the $m_{s 1}$ values are directly related to the average sulfur content $\left(\bar{X}_{s m}\right)$ values of the sampled particles; therefore, the $\sigma$ values apply to $\bar{X}_{s m}$ as well. The results are also plotted in Figure 5.29. The step-wise nature of the curves in 
Table 5.12: Values of $\boldsymbol{m}_{s 1}$ for various sample sizes based on the Model II and III results.

\begin{tabular}{|c|c|c|c|c|}
\hline$N$ & seed $=1$ & seed $=2$ & seed $=5$ & $\sigma, \%$ \\
\hline 100 & $6.75446 \times 10^{-7}$ & $6.17060 \times 10^{-7}$ & $5.83438 \times 10^{-7}$ & 7.0 \\
\hline 500 & $6.42544 \times 10^{-7}$ & $5.58211 \times 10^{-7}$ & $6.06776 \times 10^{-7}$ & 5.7 \\
\hline 1000 & $6.46193 \times 10^{-7}$ & $5.66462 \times 10^{-7}$ & $6.10643 \times 10^{-7}$ & 5.4 \\
\hline 5000 & $6.11628 \times 10^{-7}$ & $5.86313 \times 10^{-7}$ & $6.03037 \times 10^{-7}$ & 2.0 \\
\hline 10000 & $6.09172 \times 10^{-7}$ & $6.07758 \times 10^{-7}$ & $6.07282 \times 10^{-7}$ & 1.1 \\
\hline$\infty$ & $6.06776 \times 10^{-7}$ & $6.06776 \times 10^{-7}$ & $6.06776 \times 10^{-7}$ & 0.0 \\
\hline
\end{tabular}

Figure 5.29 are due to the spray zone assumption used in Model $I$, and are discussed further in Section 5.3.3.3.

Besides showing considerable scatter in the average and individual sulfur content values, the probabilities of the particles with the lower sulfur content are higher for smaller sample sizes. This trend is probably due to the small probabilities associated with particles having a high sulfur content which could easily be missed when the sample size is small. This effect appears to be more pronounced in Figure 5.29 for $N=100$.

The deviation between the measured results $(N=40)$ and the predicted results $(N=1000)$ for Run C38 (see Figure 5.24) appears similar to the deviation between the $N=50$ and $N$ $=1000$ results shown in Figure 5.30. Since Run C38 had probably reached steady state at the time of sampling (as indicated by $\bar{X}_{s} \cong \bar{X}_{s m}$ ), the deviation between the predicted and measured results probably arose from the small sample size used to determine the measured coating distribution.

\subsection{Manual Sampling}

A similar investigation was conducted for the manually sampled results. Two samples of 40 particles from Run $\mathrm{C} 17$ were analyzed for their sulfur content, and the sum of the two 


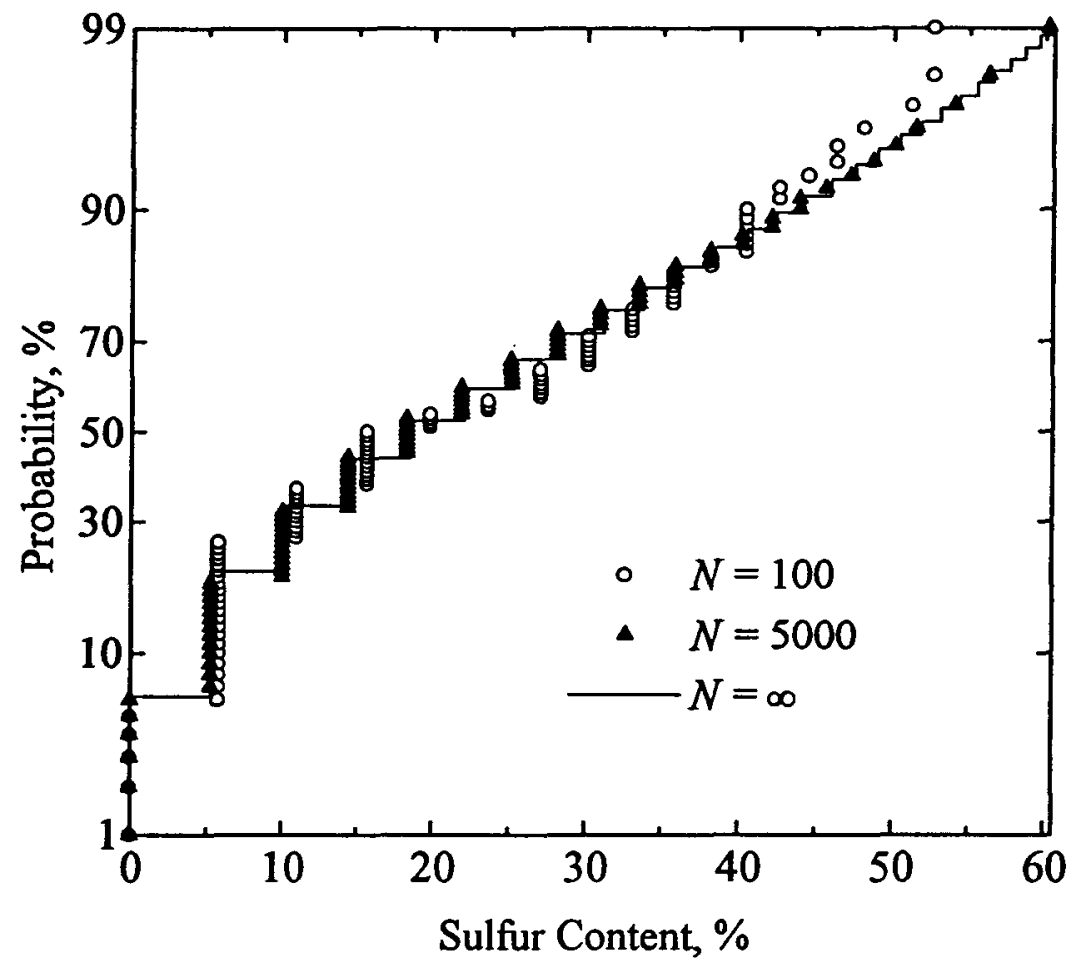

Figure 5.29: Effect of sample size on predicted coating distribution (Models II and III).

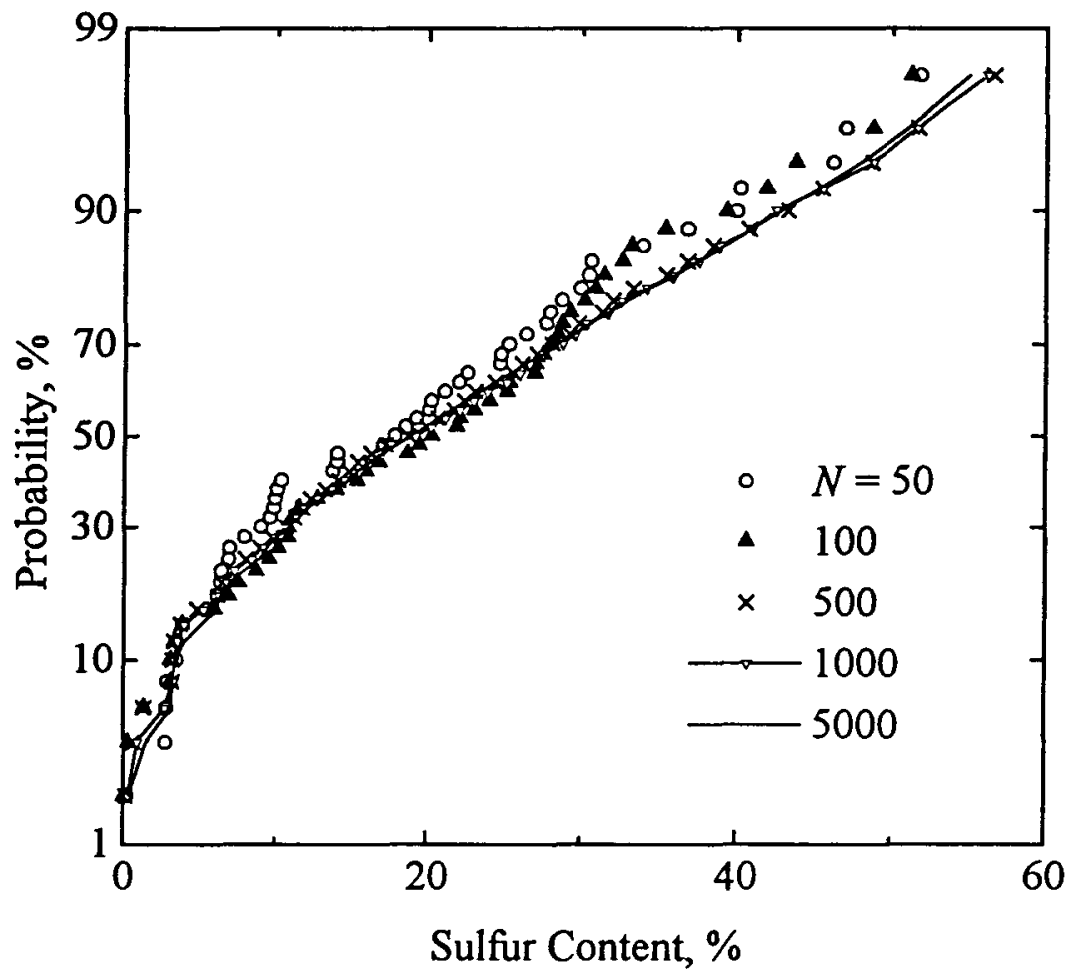

Figure 5.30: Effect of sample size on predicted coating distribution (Model IV). 
samples was used as the third sample. The results are plotted in Figure 5.31. The scatter provides some measure of the magnitude of the errors between the two sample sizes.

\subsubsection{Effect of Spray Angle}

The spray angle $(\phi)$ is a term used to define the lower height at which the coating occurs $\left(h_{f d s}\right)$ in Model IV. It should not be confused with the actual spray angle of the sulfur spray as the spray density of an actual spray varies radially while the density within $\phi$ does not. Furthermore, the effect of increasing $\phi$ may be seen as that of decreasing $h_{f d s}$.

As shown by Figure 5.32, decreasing the spray angle reduces the fraction of uncoated particles. This result is similar to the Model III results for decreasing the value of $a$, as

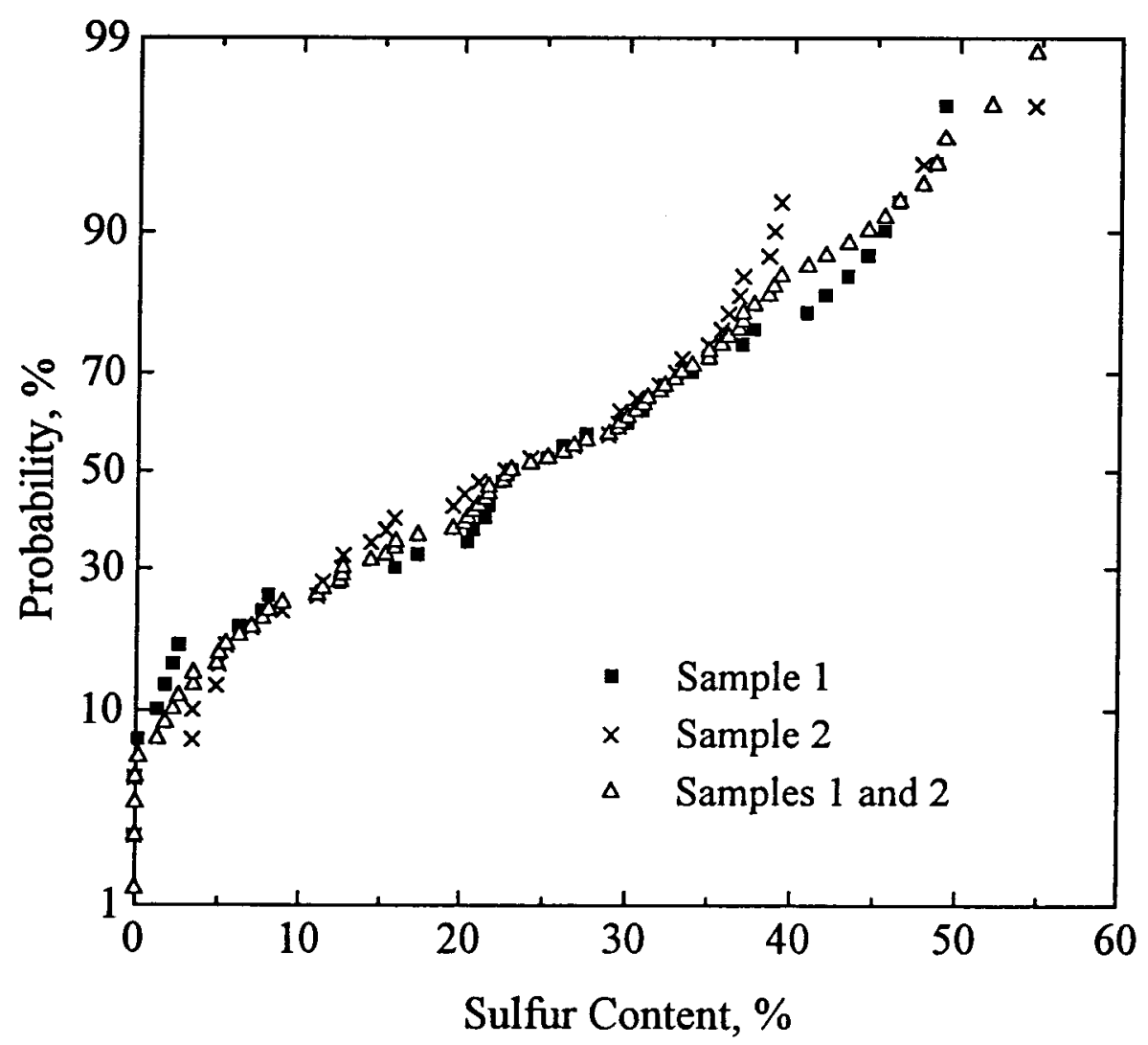

Figure 5.31: Effect of sample size (manual sampling). 


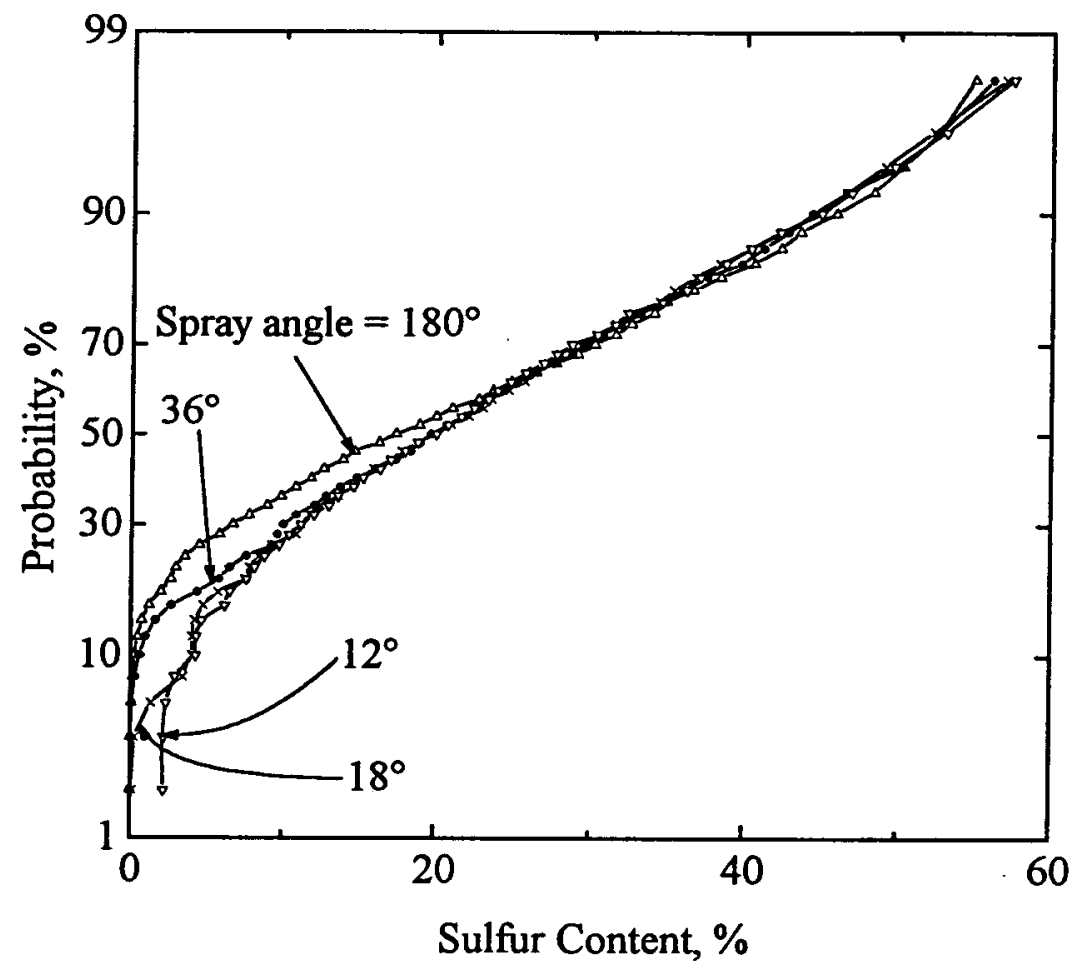

Figure 5.32: Effect of spray angle on coating distribution (results from Model IV).

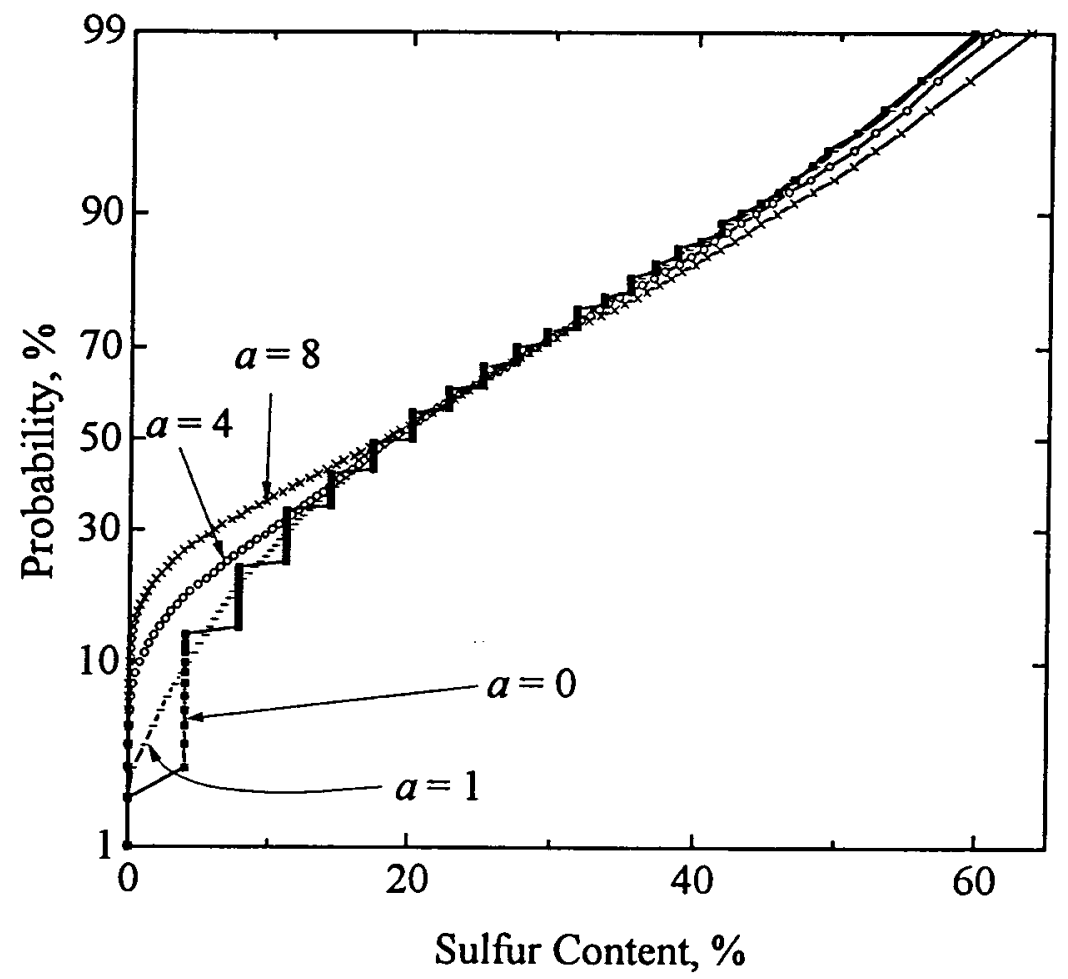

Figure 5.33: Effect of the spray concentration on coating distribution (results were obtained from Model III using Equation (4.19)). 
shown by Figure 5.33. A large value of $a$ corresponds to a rapid decrease in the spray concentration in the spray zone. As $\phi$ or $a$ increases, the size of spray zone is reduced and more particles leave the bed uncoated. The fact that a $40^{\circ}$ spray angle (i.e., equivalent to $\left.\left(h_{f d s}\right)_{\text {new }}=0.5\left(h_{f d s}\right)_{\phi=20^{\circ}}\right)$ describes the experimental data better as shown in Figure 5.24 and 5.25 , is probably due to the fact that the actual spray zone was lower than assumed. As mentioned in Section 5.3.1, this improvement was probably achieved by allowing coating to occur below the assumed $h_{f d s}$ and was not caused by an actual increase in $\phi$.

The step increases associated with the $a=0$ curve in Figure 5.33 and the curves representing smaller spray angles in Figure 5.32 resulted because a significant portion of bed particles have the same probability of receiving the same amount of coat in a discrete number of passes through the spray zone before leaving the bed. The first step represents the fraction of particles receiving one coat before leaving the bed, and subsequent steps represent the fractions of particles receiving more than one coat before leaving the bed. For $a \neq$ 0 , few bed particles have identical probabilities of passing through the same fraction of the spray zone; therefore, the steps disappear from the figures. These steps, however, can be seen in all Model II results (e.g., $N=\infty$ case in Figure 5.29).

\subsubsection{Effect of Feed Location}

Tsai (1986) claimed that the product quality improved as a result of introducing the urea feed near the wall of the bed. Particles which descend in the annulus near the wall, enter the lower portion of the spout where the spray zone is located. If all particles are forced into the spray zone, uncoated particles are avoided. However, Model II predictions in Table 5.13 indicate that the change in the coating distribution is small for $x_{e}\left(=W_{p} / W_{c}\right)=$ 0.05 . The explanation for this is that the forced feed ensures at least one pass through the spray zone, but since a small $x_{e}$ value indicates the amount of coat per cycle is very small and the total number of cycles is large, one additional cycle through the spray zone will not significantly add to the total coat on the particles. Under the operating conditions ex- 
Table 5.13: Effect of feed location (using Model II with $x_{c}=0.5, \bar{X}_{s}=0.25$ ).

\begin{tabular}{|c|c|c|c|c|c|c|c|}
\hline \multicolumn{5}{|c|}{$x_{e}=0.05$} & \multicolumn{5}{c|}{$x_{e}=0.2$} \\
\hline $\begin{array}{c}\text { Arbitrary feed } \\
\text { location }\end{array}$ & $\begin{array}{c}\text { Feed location at the } \\
\text { wall }\end{array}$ & \multicolumn{2}{c|}{$\begin{array}{c}\text { Arbitrary feed } \\
\text { location }\end{array}$} & $\begin{array}{c}\text { Feed location at the } \\
\text { wall }\end{array}$ \\
\hline$P_{k c}^{*}, \%$ & $\bar{X}_{s}, \%$ & $P_{k c}^{*}, \%$ & $\overline{X_{s}}, \%$ & $P_{k c}^{*}, \%$ & $\bar{X}_{s}, \%$ & $P_{k c}^{*}, \%$ & $\bar{X}_{s}, \%$ \\
\hline 5 & 0 & 0 & 0 & 17 & 0 & 0 & 0 \\
\hline 14 & 3 & 10 & 3 & 44 & 12 & 40 & 12 \\
\hline 22 & 6 & 19 & 6 & 63 & 21 & 64 & 21 \\
\hline 29 & 9 & 27 & 9 & 75 & 29 & 78 & 29 \\
\hline 36 & 12 & 34 & 12 & 84 & 35 & 87 & 35 \\
\hline 48 & 17 & 47 & 17 & 89 & 41 & 92 & 41 \\
\hline 57 & 21 & 57 & 21 & 93 & 45 & 95 & 45 \\
\hline 65 & 25 & 65 & 25 & 95 & 49 & 97 & 49 \\
\hline 71 & 29 & 72 & 29 & 97 & 52 & 98 & 52 \\
\hline 77 & 32 & 77 & 32 & 98 & 55 & 99 & 55 \\
\hline 81 & 35 & 81 & 35 & 99 & 58 & 99 & 58 \\
\hline 84 & 38 & 85 & 38 & 99 & 60 & 100 & 60 \\
\hline 87 & 41 & 88 & 41 & 99 & 62 & 100 & 62 \\
\hline 90 & 44 & 91 & 44 & 100 & 64 & 100 & 64 \\
\hline & & & $P_{k c}^{*}=$ cumulative probability & & \\
\hline
\end{tabular}

amined in this study, $x_{e}$ was less than 0.05 and only small changes were observed in the coating distribution for various feed locations. The effect of the feed location on the coating distribution for the conditions of Run C17 can be seen in Figure 5.34.

\subsubsection{Effect of Beds-in-Series}

The greatest improvement in the coating distribution can be achieved by operating several beds-in-series, as shown by Figures 4.2 and 5.35. Models I and IV both predict drastic reduction in uncoated and heavily coated fractions for large numbers of beds-in-series; the distributions approach those of the batch products (see Figure 4.3). These improvements in the product are of commercial importance and are discussed further in Section 5.4. (In Model IV, one bed size was used regardless of number of beds-in-series.) 


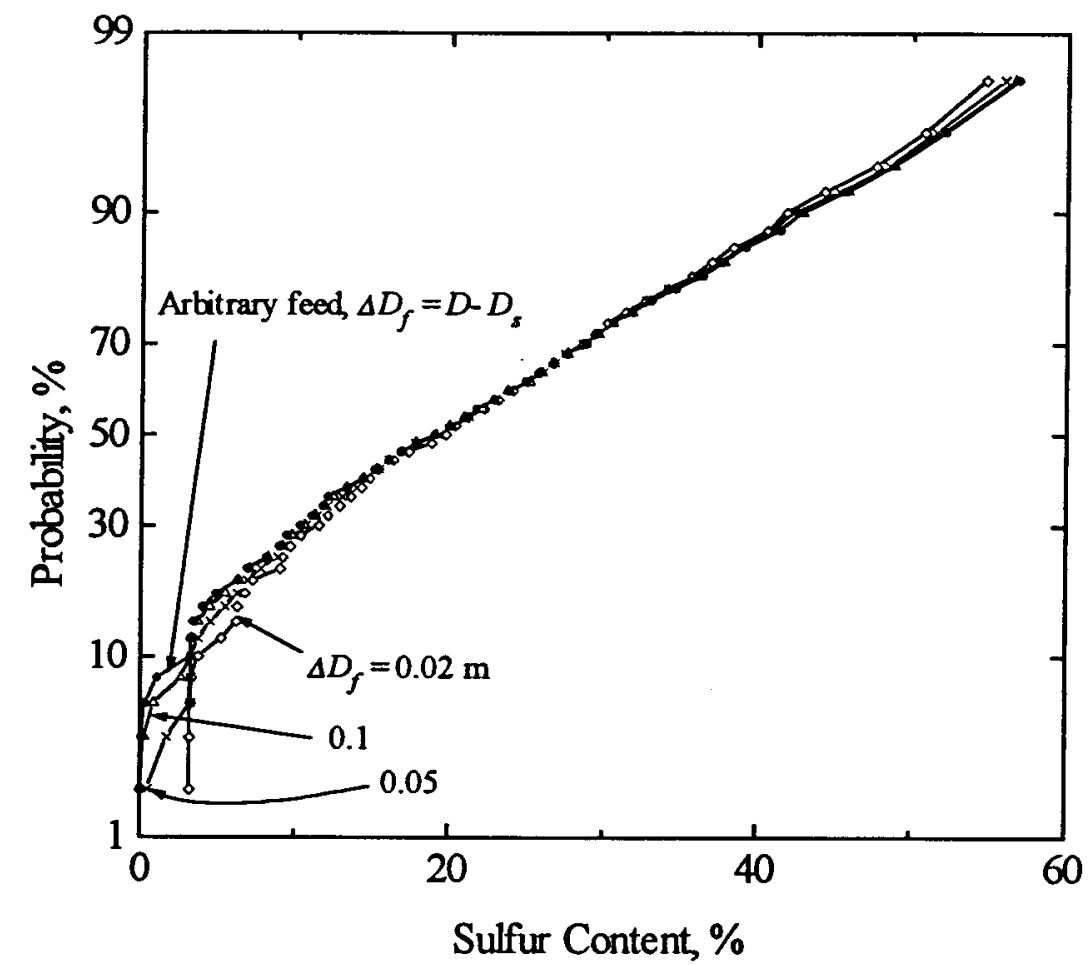

Figure 5.34: Effect of feed location (results from Model IV).

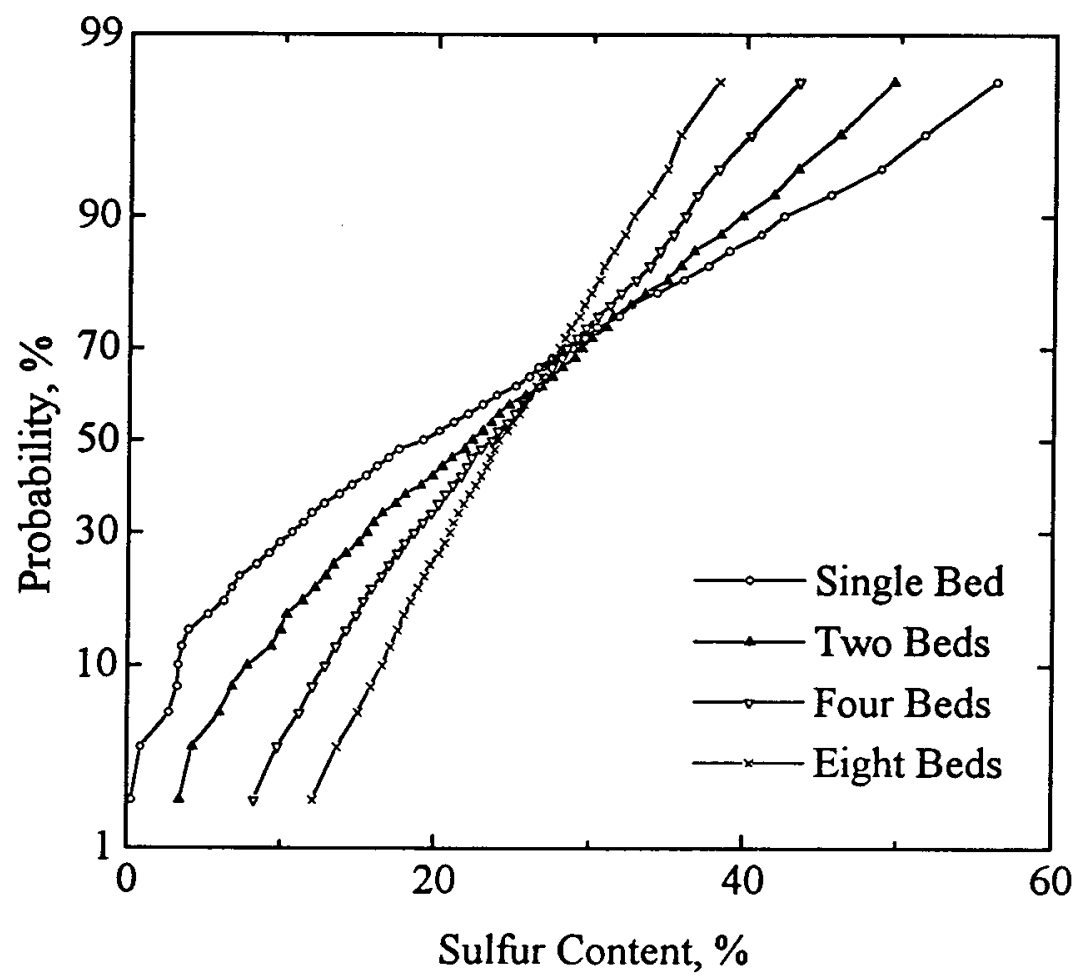

Figure 5.35: Product coating distributions for beds-in-series (from Model IV). 


\subsubsection{Effect of Model Variables, $x_{e}$ and $x_{c}$}

The variables $x_{c}$ and $x_{e}$ in Models II and III characterize two main factors influencing the coating distribution. Their relationships to the operating variables were not determined in this work as a large number of experiments are required (see Chapter 4); however, the effect of operating variables on these model variables can be explained qualitatively. The size of the spray zone is characterized by $x_{c}$, and increasing $x_{c}$ is equivalent to increasing the spray zone or reducing the by-pass zone. The number of times the particles pass through the spray zone is characterized by $x_{e}\left(=W_{p} / W_{c}\right)$, and increasing $x_{e}$ is equivalent to increasing the particle discharge rate, decreasing the circulation rate, or both.

The operating variables that affect $x_{c}$ are $Q_{s}, Q_{a}, W_{s}$ and $\phi . Q_{s}$ determines the impaction velocity of the droplets onto the bed particles and influences both $\eta$ and $N_{i}$ in Equations (2.26) and (4.34). $Q_{a}$ and $W_{s}$ determine the droplet size and influence $\eta$ in Equation (2.26). $\phi$ influences the spray zone directly. The value of $x_{c}$ increases with $Q_{a}$ and decreases with $Q_{s}, W_{s}$ and $\phi$.

The operating variables that affect $x_{e}$ are $W_{p}$ and $W_{c} . W_{p}$ is directly affected by $W_{u}$ and $W_{c}$ is influenced by $Q_{s}, H, D$ and $d_{i}$. $W_{c}$ increases with $Q_{s}, H, D$ and $d_{i}$. The value of $x_{e}$ increases with $W_{p}$ and decreases with $W_{c}$.

The effects of $x_{c}$ and $x_{e}$ on coating distribution are shown in Figures 5.36 and 5.37. The results presented in Figure 5.36 indicate that for $x_{e}=0.05$ and $x_{c}=0.6$, over $30 \%$ of particles receive three coats or less. Three coats on a particle are likely not enough to completely cover the particle. To increase the number of coats on the particles, $x_{e}$ must be decreased and $x_{c}$ increased (see Figure 5.37).

The variables $x_{e}$ and $x_{c}$ can be varied to simulate various types of beds. These variables are also useful in predicting the presence of uncoated urea particles in the product stream. 


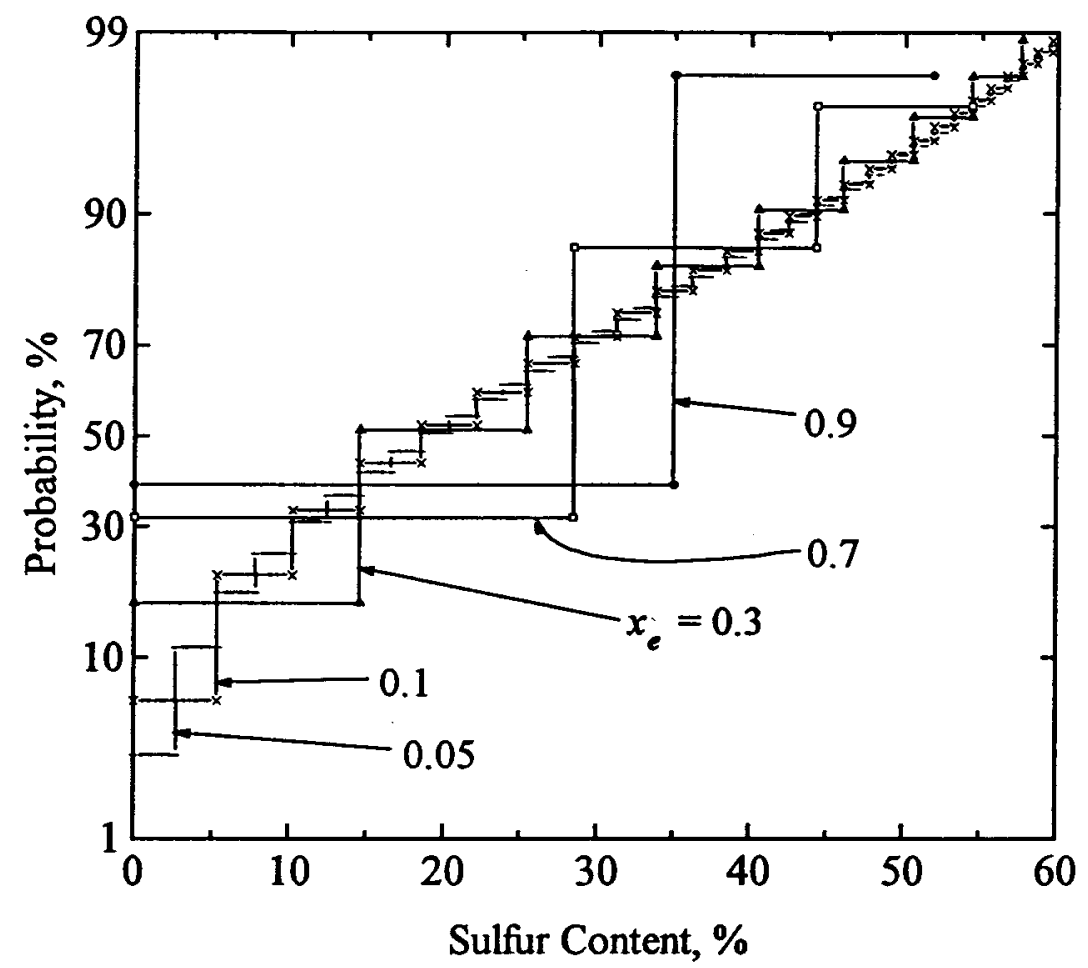

Figure 5.36: Sensitivity analysis of $x_{e}$ on coating distribution using Model II $\left(x_{c}=0.6\right)$.

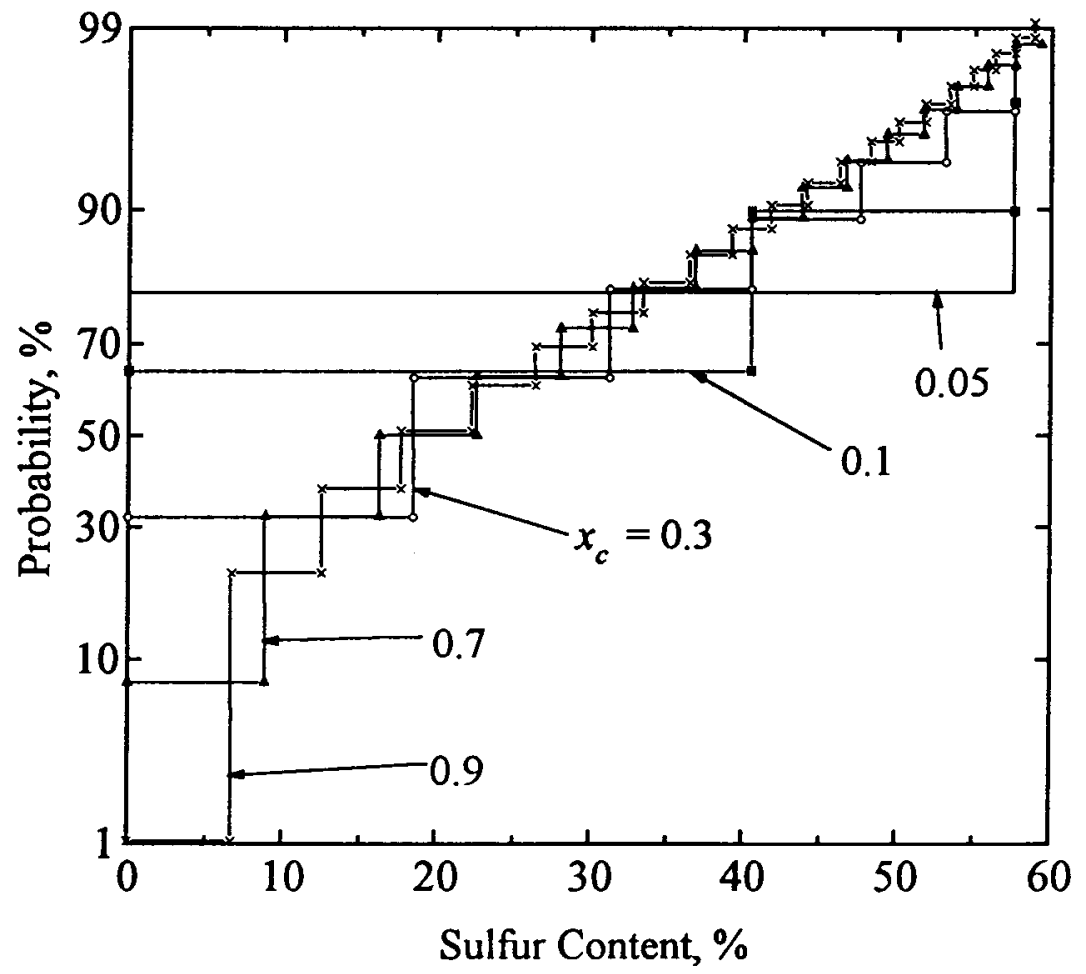

Figure 5.37: Sensitivity analysis of $x_{c}$ on coating distribution using Model II $\left(x_{e}=0.2\right)$. 
Both plug flow and CST residence time models can be represented by manipulating the $x_{e}$ and $x_{c}$ values. Plug flow is simulated when $x_{e}=1$ which is equivalent to a spouted bed equipped with a draft tube which also serves as a discharge tube. Continuous stirred tank behavior is simulated when $x_{e}=0$. Model I predictions can be duplicated by Model II when $x_{c}=1$. However, a finite fraction of uncoated urea particles in the product stream is only predicted when $x_{c}<1$. Note also that Model III becomes Model II when $a=0$.

\subsubsection{Sensitivity Analysis Using Model IV}

Certain operating variables $\left(Q_{s}, Q_{a}, d_{i}, H, D\right.$ and $\left.W_{p}\right)$ had little effect on the coating distribution and were not discussed in the previous section. In this section, the effects of all the principal variables are examined. Model IV provided good predictions of the measured data (see Figures 5.23 - 5.25 and 5.27); therefore, its results are used to identify the main variables influencing the product quality.

The principal factor affecting the product quality is the sulfur content. The amount of sulfur deposited on the urea product indicates the thickness of coating; the quality of the coat, in turn, determines the rate of urea dissolution. The model and experimental results in Figure 5.38 clearly indicate that the sulfur content is the major factor influencing the product quality.

Since the sulfur content is the dominant factor influencing the product quality, and because Model I is based only on $\bar{X}_{s}$, Model I should also provide a good estimate of the results. As shown by Figure 5.38, Model I does give a good estimate of Model IV results, and both models agree well with the experimental results regardless of the condition (steady state or transient) under which the samples were collected. This result is not surprising since the value of $x_{e}$ is very small for the operating conditions considered in this study. The discrepancy between the predicted and measured results are likely due to the presence of significant amounts of uncoated urea in the bed under transient conditions, as all coat- 


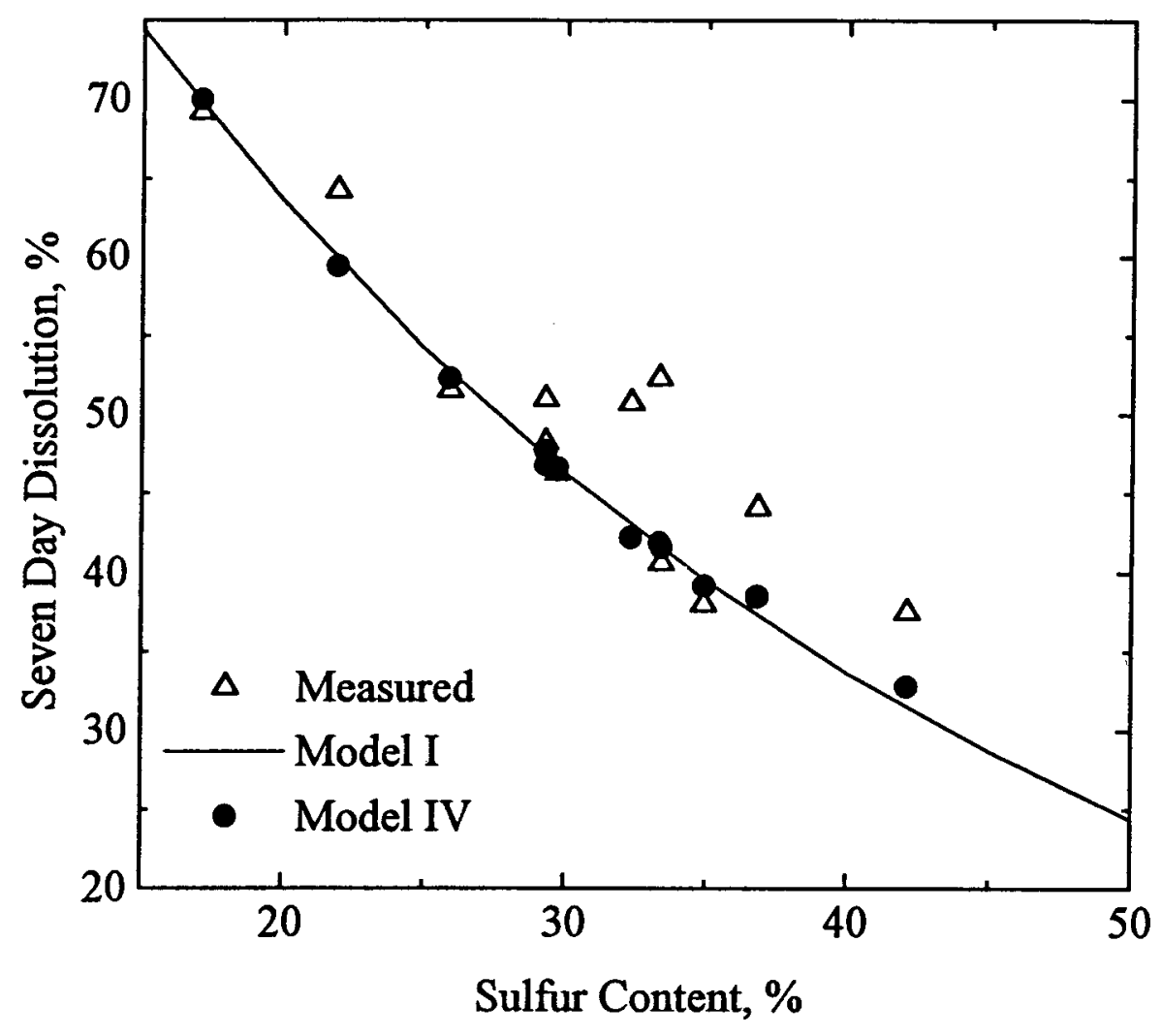

Figure 5.38: Model predictions of product quality.

ing operations started with uncoated urea and insufficient time was allowed for the bed to reach steady state.

The differences between the Model I and Model IV predictions are attributed to the effects of other operating variables on the product quality. To determine the effects of the principal operating variables, a sensitivity analysis was conducted. The sulfur content and the bed temperature were fixed at $25 \%$ and $60^{\circ} \mathrm{C}$, respectively, and only one variable was changed at a time. The range of model variables examined are given in Table 5.14 and the results are shown in Table 5.15. The same base condition was not used for all runs as a change in one variable often resulted in a change in other variables (e.g., an increase in the urea feed rate required an increase in the sulfur feed rate to produce $25 \%$ sulfur coated urea). $U / U_{m s}$ values of either 1.1 or 1.2 were used in all runs, unless otherwise specified. 
Table 5.14: Range of model variables examined for sensitivity analysis.

\begin{tabular}{|c|c|}
\hline Variable & Operating Range \\
\hline Spouting air velocity, $U / U_{m s}$ & $1.05-1.4$ \\
\hline Atomizing air flow rate, $Q_{a}$ & $0.44-0.78 \mathrm{~L} / \mathrm{s}$ \\
\hline Bed Height, $H$ & $0.25-0.55 \mathrm{~m}$ \\
\hline Bed Diameter, $D$ & $0.25-0.45 \mathrm{~m}$ \\
\hline Spouting air orifice diameter, $d_{i}$ & $21.2-28.2 \mathrm{~mm}$ \\
\hline Urea feed rate, $W_{u}$ & $6.43-22.9 \mathrm{~g} / \mathrm{s}$ \\
\hline Spray angle, $\phi$ & $12-180^{\circ}$ \\
\hline Bed particle size, $d_{n}$ & $2.1-4.3 \mathrm{~mm}$ \\
\hline Feed location, $\Delta D_{f}$ & $0.02 \mathrm{~m}-$ Arbitrary \\
\hline Sample size, $N$ & $50-5000$ particles \\
\hline Number of beds in series, $N_{\text {heds }}$ & $1-8$ beds \\
\hline
\end{tabular}

The range of model variables studied actually exceeded the range that was verified for the hydrodynamics study and, as a result, the coating model predictions may not provide accurate results for certain experimental conditions (e.g., for deep beds and large orifice sizes).

The results given in Table 5.15 show that the maximum change in $U_{D}$ is less than $4 \%$ for a single bed. Such a small change in the product quality may be explained by examining the effect of each operating variable on coating distribution; however, the effect of most operating variables may be explained more easily by considering the effects of Model II variables $x_{e}$ and $x_{c}$ (see Section 5.3.3.6). In this manner, the effects of many operating variables on the coating distribution can be simplified to the effect of just the two model variables.

For the operating conditions examined, $x_{e}<0.1$. For such small values of $x_{e}$, the effect of $x_{c}$ on the coating distribution is noticeable (see Figure 5.39) only for the inadequately coated $\left(\bar{X}_{s}<15 \%\right)$ and heavily coated $\left(\bar{X}_{s}>30 \%\right)$ particles, i.e., little change in the 
Table 5.15: Results of sensitivity analysis using Model IV $\left(\bar{X}_{s}=0.25, T=60^{\circ} \mathrm{C}\right)$.

\begin{tabular}{|c|c|c|c|c|c|c|c|}
\hline \multicolumn{2}{|c|}{ Bed Diameter } & \multicolumn{2}{|c|}{ Atomizing Air } & \multicolumn{2}{|c|}{ Urea Feed Rate } & \multicolumn{2}{|c|}{ Orifice Diameter } \\
\hline$D, \mathrm{~m}$ & $U_{D}, \%$ & $Q_{a}, \mathrm{~L} / \mathrm{s}$ & $U_{D}, \%$ & $W_{w}, \mathrm{~g} / \mathrm{s}$ & $U_{D}, \%$ & $d_{i}, \mathrm{~mm}$ & $U_{D}, \%$ \\
\hline 0.25 & 54.2 & 0.44 & 52.5 & 6.43 & 53.9 & 21.2 & 53.4 \\
\hline 0.35 & 53.6 & 0.61 & 52.2 & 12.3 & 54.2 & 24.7 & 54.2 \\
\hline 0.45 & 53.3 & 0.78 & 52.0 & 22.9 & 54.3 & 28.2 & 53.9 \\
\hline \multicolumn{2}{|c|}{ Bed Height } & \multicolumn{2}{|c|}{ Spouting Air } & \multicolumn{2}{|c|}{ Bed Particle Id. } & \multicolumn{2}{|c|}{ Spray Angle } \\
\hline$H, \mathrm{~m}$ & $U_{D}, \%$ & $U / U_{m s}$ & $U_{D}, \%$ & $d_{n}, \mathrm{~mm}$ & $U_{D}, \%$ & $\phi$ & $U_{D}, \%$ \\
\hline 0.25 & 53.9 & 1.05 & 54.7 & 2.1 & 54.5 & $180^{\circ}$ & 56.0 \\
\hline 0.35 & 53.9 & 1.1 & 54.2 & 3.2 & 54.4 & $36^{\circ}$ & 52.9 \\
\hline 0.45 & 54.2 & 1.2 & 54.4 & 4.3 & 54.6 & $18^{\circ}$ & 52.5 \\
\hline 0.55 & 54.2 & 1.4 & 53.7 & & & $12^{\circ}$ & 52.7 \\
\hline \multicolumn{8}{|c|}{ Feed Location } \\
\hline$\Delta D_{f}, \mathrm{~m}$ & 0.02 & 0.05 & 0.1 & 0.15 & $D-D_{s}$ & & \\
\hline$U_{D}, \%$ & 53.4 & 53.9 & 54.2 & 54.3 & 54.3 & & \\
\hline \multicolumn{8}{|c|}{ Sample Size } \\
\hline$N$ & 50 & 100 & 500 & 1000 & 5000 & & \\
\hline$U_{D}, \%$ & 58.4 & 53.3 & 54.9 & 54.0 & 54.2 & & \\
\hline \multicolumn{8}{|c|}{ Beds in Series } \\
\hline$N_{\text {beds }}$ & 1 & 2 & 4 & 8 & & & \\
\hline$U_{D}, \%$ & 54.0 & 46.4 & 40.8 & 35.9 & & & \\
\hline \multicolumn{8}{|c|}{ Model I Prediction: $U_{D}=54.2 \%$} \\
\hline
\end{tabular}

range $15 \%<\bar{X}_{s}<30 \%$ is observed for $x_{e}=0.05$, even though the $x_{c}$ value is quite different. This change in the coating distribution does not change the product quality significantly because the product quality is determined primarily by the fraction of particles having $15 \%<\bar{X}_{s}<30 \%$ (see Figure 5.26 or Equation 5.20 ).

The most significant improvement in product quality occurs when urea is coated in a series of beds. This result is consistent with the above explanation that the fraction in the range 


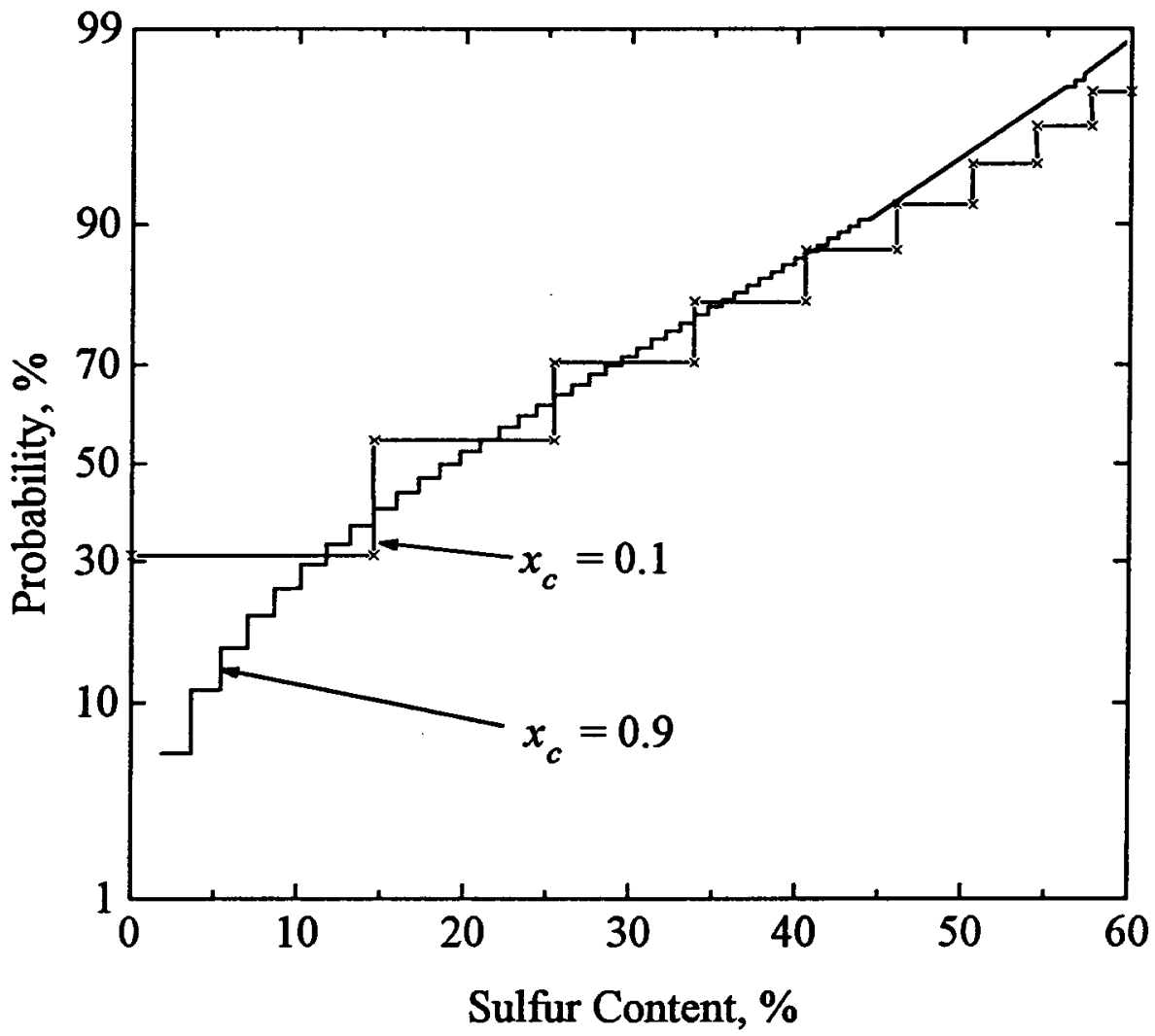

Figure 5.39: Effect of a large change in $x_{c}$ on coating distribution $\left(x_{e}=0.05\right)$.

$15 \%<\bar{X}_{s}<30 \%$ determines the product quality. Figure 5.35 clearly shows that this fraction becomes larger as the number of beds increases.

Since Model I provides an accurate prediction of the fraction in the range $15 \%<\bar{X}_{s}<30$ $\%$ (see Figure 5.24), it also provides an accurate prediction of the product quality (see Table 5.15). In addition, the Model I solution can be obtained easily; hence, it may be useful in obtaining quick and accurate estimates of the product quality. However, it should be noted that the inadequately coated and heavily coated fractions are also important (e.g., to the appearance of the product and design specifications which require information on the maximum bed particle size) and the Model I predictions of these fractions may be poor. 
The effect of sample size can also be seen in Table 5.15. The purpose of including these values was to check the error associated with the sample size. The results in terms of the deviations from the value obtained for 5000 particles are similar to those of the standard deviations calculated for the coating distribution (see Table 5.12).

\subsection{Commercial Implications}

The findings of this study are applicable to the design of commercial coating plants. Model I provides a quick estimate of the product quality suitable for preliminary plant designs, while Model IV may be used for more detailed studies. Models II and III can aid with conceptualizing spouted bed coating processes.

Commercial plants will normally have considerably higher capacities than the current apparatus. Higher capacities may be obtained in two ways: increasing the bed size and increasing the number of beds in parallel. The latter option should be considered only if the former option does not provide the desired capacity, because an increase in the number of beds can pose operational difficulties. These include controlling the distributions of air and liquid sulfur to different beds. Larger beds, on the other hand, may increase the production rate without sacrificing the product quality (presuming the hydrodynamics and $x_{e}$ stay constant).

It is unlikely that the product quality can be controlled by varying the production rate alone. As discussed already, the product quality is determined primarily by the number of beds in series. The product quality increases with the number of beds-in-series, but because operational difficulties also increase, the minimum number of beds that meets the design quality specification should be used. Note that the increase in the product quality is large when the number of beds increases from one to two beds, but the increase in the product quality becomes smaller for each additional bed. 


\section{Chapter 6. \\ Conclusions and Recommendations}

\subsection{Conclusions}

The spouted bed coating process for the batch-wise and continuous production of sulfur coated urea has been successfully modeled using mass and momentum balance equations and inertial sulfur droplet deposition as the dominating coating mechanism.

Specific conclusions that may be drawn from the results given in Chapter 5 are listed in the following sub-sections.

\subsubsection{Bed Hydrodynamics}

- The minimum spouting velocity $\left(U_{m s}\right)$ varies linearly with the bed height in conical beds.

- $U_{m s}$ varies proportionally with the total momentum due to atomizing air and spouting air flow into the bed.

- $U_{m s}$, axial pressure profile in the annulus and air velocity in the spout in conical beds are similar to the corresponding quantities in conical-cylindrical beds with the column diameter equal to $80 \%$ of the maximum diameter of conical beds.

- $U_{m s}$ for shallow beds could be well represented by the correlation given by Equation (5.4).

- The Morgan-Littman (1980) correlation provides accurate axial pressure profiles in the annulus for shallow spouted beds if the diameter modification for conical beds is used. 
- The mass and momentum balances suggested by Lefroy and Davidson (1969) give accurate predictions of air velocity in the spout, provided that the vector form of the Ergun (1952) equation for gas flow in the annulus and the modifications listed above are used.

\subsubsection{Spray Studies}

- Consistent sulfur atomization by the nozzle used in this work occurs in the range defined by Equations (5.8) and (5.9).

- The size of the sulfur droplets typically ranged from 6 to $50 \mu \mathrm{m}$ dia. and is well represented by a log-normal distribution.

- The average droplet size is affected primarily by the sulfur feed rate, and is well represented by the power-law correlation given by Equation (5.17).

- The dominant mechanism by which the sulfur droplets deposit onto the bed particles is inertial impaction.

\subsubsection{Coating Distribution and Product Quality}

- The spray angle, urea feed location and the number of beds-in-series significantly affect the amount of lightly coated and heavily coated particles in the sulfur coated urea product.

- The product quality is primarily affected by the average sulfur content of the product and the number of beds-in-series. Spray angle, feed location, atomizing and spouting air flow rates, urea and sulfur feed rates, and bed diameter also affect the product quality, but to a much lesser extent.

- The Monte Carlo method provides good representation of coating distribution if a sufficient number of particles are simulated.

- The spouted bed coating unit is capable of producing sulfur coated urea of widely varying quality at a high production rate. 


\subsection{Recommendations for Further Work}

Certain difficulties and assumptions in modeling the coating process resulted from the limitations of the one-dimensional models used in this work. A significant number of assumptions could be reduced if a fully two-dimensional (axi-symmetric) model is developed. For example, $h_{f d s}$ would not have to be assumed because the particle concentration and the spray distribution in the spout could be determined in a two-dimensional model. Some experimental work may be necessary to develop a model of the radial spray density in the spray region. The model should also be a three-phase model as the effects of sulfur spray on the bed hydrodynamics cannot be properly determined with the two-phase model developed in this work. The two-dimensional, two-phase model developed by Krzywanski (1992) would be a good starting point for such a model.

Before commercialization of the spouted bed process, a pilot plant consisting of several beds-in-series should be evaluated. Amongst the foreseeable problems are control of the distribution of air and sulfur to different beds, transportation of large particles from bed to bed, and separation of large particles from the product. These problems cannot be addressed until a pilot plant is actually available. A continuous sulfur supply system should also be incorporated into the pilot plant to study the effects of operating time.

The hydrodynamics of larger diameter beds should also be examined because many correlations used in this work may not apply to larger beds (Lim and Grace, 1987).

Finally, the sulfur droplet distribution in the spouted bed could not be directly measured using the devices examined in this work. A further study on such a device could be valuable and it would advance aerosol detection in complex environments. The devices studied here could be a good starting point for such an effort. 


\section{Nomenclature}

\begin{tabular}{|c|c|}
\hline$A_{a s}$ & Local spray area, $\mathrm{m}^{2}$ \\
\hline$A_{0}$ & Area of shutter opening, $\mathrm{m}^{2}$ \\
\hline$A_{p}$ & Projected area of spout particles per unit volume of spout, $\mathrm{m}^{-1}$ \\
\hline$A_{p}^{*}$ & Projected area (cross-sectional area) of a particle, $\mathrm{m}^{2}$ \\
\hline$A_{s}$ & Spout cross-sectional area, $\mathrm{m}^{2}$ \\
\hline$a$ & Exponent in Equation (4.17), dimensionless \\
\hline$C$ & Concentration of sulfur droplets in the gas phase, $\mathrm{kg} / \mathrm{m}^{3}$ \\
\hline$C_{c}$ & Spray concentration defined for Model III, $\mathrm{kg} / \mathrm{m}^{3}$ \\
\hline$C_{o}$ & Pitot tube coefficient, dimensionless \\
\hline$c$ & Fitted coefficient of $D$ in Equation (5.3) \\
\hline 8 & Diffusion Coefficient, $\mathrm{m}^{2} / \mathrm{s}$ \\
\hline$D$ & Column diameter, $\mathrm{m}$ \\
\hline$D\{z\}$ & Local bed diameter, $\mathrm{m}$ \\
\hline$D^{\prime}$ & $\begin{array}{l}\text { "Effective" bed diameter }\left(D^{\prime}=0.8 D_{m} \text { in conical section and } D^{\prime}=D \text { in }\right. \\
\text { conical-cylindrical section), } \mathrm{m}\end{array}$ \\
\hline$D_{25}$ & $\begin{array}{l}\text { Seven day urea dissolution for products with } 25 \% \text { sulfur content, } \\
\text { fractional }\end{array}$ \\
\hline$D_{a s}$ & Local diameter of the spray, $m$ \\
\hline$D_{f}$ & $D-\Delta D_{f} \mathrm{~m}$ \\
\hline$D_{i n i}$ & Location of feed particle on the surface of the bed during its first cycle, $m$ \\
\hline$D_{m}$ & Maximum annulus diameter for the conical bed, $m$ \\
\hline$D_{r}$ & Location of bed particles on the surface of the bed, $m$ \\
\hline$D_{s}$ & Spout diameter, $m$ \\
\hline$d_{a}$ & Diameter of atomizing air nozzle cap at top, $m$ \\
\hline
\end{tabular}


$d_{a t} \quad$ Diameter of nozzle orifice, $\mathrm{m}$

$d_{c} \quad$ Diameter of aerosol particle, $\mathrm{m}$

$d_{g c} \quad$ Geometric counted mean of droplet size, $m$

$d_{i} \quad$ Orifice diameter based on the flow area of $Q_{s}, \mathrm{~m}$

$d_{i}^{\prime} \quad$ Corrected orifice diameter in Equation (5.2), $\mathrm{m}$

$d_{o} \quad$ Shutter diameter based on the flow area, $m$

$d_{p} \quad$ Average diameter of bed particle, $\mathrm{m}$

$d_{p i} \quad$ Mean diameter of adjacent Tyler screen apertures, $m$

$d_{s} \quad$ Diameter of sulfur spray droplets, $\mathrm{m}$

$d_{s v} \quad$ Sauter mean (surface-volume) diameter, $\mathrm{m}$

$F\{t\} \quad$ Distribution function of residence time, dimensionless

$f_{1}, f_{2} \quad$ Coefficients of Ergun equation, $\mathrm{kg} / \mathrm{m}^{3} \mathrm{~s}$ and $\mathrm{kg} / \mathrm{m}^{4}$

$E_{D}, E_{D T}$, Collection efficiency due to diffusion, direct interception, gravitation and

$E_{G}, E_{I} \quad$ inertial impaction, dimensionless

$G \quad \rho U$

$G\left\{X_{s}\right\} \quad$ Exit sulfur content distribution, dimensionless

$g \quad$ Acceleration due to gravity, $\mathrm{m} / \mathrm{s}^{2}$

$H \quad$ Loosely packed static bed height, $\mathrm{m}$

$H_{c} \quad$ Height of cone base, $\mathrm{m}$

$H_{m} \quad$ Maximum spoutable bed depth, $\mathrm{m}$

h $\quad H / H_{m}$

$h_{f d s} \quad$ Height at which the spray coating begins, $m$

$k \quad$ Coefficient in Equation (2.3); number of passes through the spray zone

$k_{c} \quad$ Proportionality constant in Equation (4.19)

$k_{p} \quad$ Probability constant in Equation (4.20), $\mathrm{m}^{-1}$

$k_{\theta} \quad$ Angle of fully developed spray jet, ${ }^{\circ}$

$M_{t} \quad$ Momentum flow of spouting and atomizing air into the bed, $\mathrm{kg} \cdot \mathrm{m} / \mathrm{s}^{2}$ 


\begin{tabular}{|c|c|}
\hline$m_{p}$ & Total weight of the bed particles, $\mathrm{kg}$ \\
\hline$m_{s}$ & Weight of sulfur in sulfur coated urea, $\mathrm{kg}$ \\
\hline $\bar{m}_{s}$ & Mean weight of sulfur in sulfur coated urea, $\mathrm{kg}$ \\
\hline$m_{s}^{*}$ & Conditional weight of sulfur deposited on urea, $\mathrm{kg}$ \\
\hline$m_{s l}$ & $\begin{array}{l}\text { Amount of sulfur deposited on a bed particle per pass through the spray } \\
\text { zone, } \mathrm{kg}\end{array}$ \\
\hline$\overline{\boldsymbol{m}}_{\mathrm{sl}}$ & Mean weight of sulfur picked up per pass through spray zone, $\mathrm{kg}$ \\
\hline$m_{k}$ & Weight of urea in sulfur coated urea, $\mathrm{kg}$ \\
\hline$\overline{\boldsymbol{m}}_{\boldsymbol{u}}$ & Mean weight of urea in sulfur coated urea, $\mathrm{kg}$ \\
\hline$N$ & Sample size, particles; number of cycles, cycles \\
\hline$N_{a}^{*}$ & Deposition rate of sulfur droplets in the annulus between streamlines, $\mathrm{kg} / \mathrm{s}$ \\
\hline$N_{D I}$ & Interception parameter, dimensionless \\
\hline$N_{G}$ & Gravitational settling parameter, dimensionless \\
\hline$N_{i}$ & $\begin{array}{l}\text { Deposition rate of sulfur droplets on spout particles per unit volume of } \\
\mathrm{spout,} \mathrm{kg} / \mathrm{s} \cdot \mathrm{m}^{3}\end{array}$ \\
\hline$N_{R}$ & Numerically generated random number between 0 and 1 \\
\hline$n$ & Number of beds in series in Equation (4.7) \\
\hline $\bar{n}$ & Direction normal to streamlines, $\mathrm{m}$ \\
\hline$P$ & Pressure, $\mathrm{Pa}$ \\
\hline$P_{f}$ & Pressure in fluidized bed, $\mathrm{Pa}$ \\
\hline$P_{a}\{z\}$ & Pressure at $z, \mathrm{~Pa}$ \\
\hline $\operatorname{Pr}\{z\}$ & Probability of particle entering at $z$ into the spray zone, fractional \\
\hline$P_{k c}$ & Probability of particle entering the spray zone $k$ times, fractional \\
\hline$Q_{a}$ & Volumetric atomizing air flow rate, $\mathrm{m}^{3} / \mathrm{s}$ \\
\hline$Q_{l}$ & Flow rate of spray liquid, $\mathrm{m}^{3} / \mathrm{s}$ \\
\hline$Q_{m a}$ & Atomizing air flow rate at minimum atomization, $\mathrm{m}^{3} / \mathrm{s}$ \\
\hline$Q_{m s}$ & Volumetric flow rate at minimum spouting, $\mathrm{m}^{3} / \mathrm{s}$ \\
\hline
\end{tabular}


$Q_{s} \quad$ Volumetric spouting air flow rate, $\mathrm{m}^{3 / \mathrm{s}}$

$Q_{t} \quad$ Total volumetric flow rate $=Q_{a}+Q_{s} \mathrm{~m}^{3 / \mathrm{s}}$

$r \quad$ Radial distance from the centre of the bed, $m$

$S \quad$ Distance along the stream line, $\mathrm{m}$

$S_{d} \quad$ Standard deviation of $U_{m s}$ predictions, $\mathrm{m} / \mathrm{s}$

$S_{t} \quad$ Stoke's number, dimensionless

$T, T_{b} \quad$ Bed temperature, ${ }^{\circ} \mathrm{C}$

$t \quad$ Time, $s$

$\bar{t} \quad$ Mean residence time of solids, $s$

$t_{c} \quad$ Cycle time, $s$

$t_{r} \quad$ Residence time, $s$

$U \quad$ Superficial air velocity, $\mathrm{m} / \mathrm{s}$

U Vector form of air velocity, $\mathrm{m} / \mathrm{s}$

$U_{D} \quad$ Predicted seven day urea dissolution rate, fractional

$U_{D m} \quad$ Measured seven day urea dissolution rate, fractional

$U_{m s} \quad$ Minimum spouting velocity based on column diameter $(D), \mathrm{m} / \mathrm{s}$

$U_{m s}^{\prime} \quad$ Minimum spouting velocity based on $D^{\prime}, \mathrm{m} / \mathrm{s}$

$U_{r} \quad$ Volumetric rate of air cross-flow per unit area of spout-annulus interface, $\mathrm{m} / \mathrm{s}$

$U_{t} \quad$ Terminal settling velocity, $\mathrm{m} / \mathrm{s}$

$u \quad$ Interstitial air velocity, $\mathrm{m} / \mathrm{s}$

$u_{r} \quad$ Relative velocity between gas and liquid at the orifice, $\mathrm{m} / \mathrm{s}$

$V_{b} \quad$ Total bed volume, $\mathrm{m}^{3}$

$V_{r} \quad$ Entrainment rate of bed particles into the spout per unit area of spoutannulus interface, $\mathrm{m} / \mathrm{s}$

$v \quad$ Local bed particle velocity, $\mathrm{m} / \mathrm{s}$

$W_{c} \quad$ Circulation rate of bed particles, $\mathrm{kg} / \mathrm{s}$ 


$\begin{array}{ll}W_{p} & \text { Production rate, } \mathrm{kg} / \mathrm{s} \\ W_{s} & \text { Sulfur injection rate, } \mathrm{kg} / \mathrm{s} \\ W_{u} & \text { Urea feed rate, } \mathrm{kg} / \mathrm{s} \\ X & \text { Defined in Equation (2.13), dimensionless } \\ X_{s} & \text { Sulfur content, dimensionless } \\ \bar{X}_{s} & \text { Mean sulfur content of sulfur coated urea based on urea and sulfur feed } \\ \bar{X}_{s m} & \text { rates, fractional } \\ \bar{X}_{s p} & \text { Mean sulfur content of sulfur coated urea based on a crush test, fractional } \\ X_{s p, i} & \text { Mean sulfur content of simulated (SCU) particles, fractional } \\ x & \text { Sulfur content of individual particle, fractional } \\ x_{b} & z / H_{m} \\ x_{c} & \text { Probability of particle by-passing the spray zone, fractional } \\ x_{e} & \text { Probability of particle entering the spray zone, fractional } \\ x_{f} & \text { Probability of particle exitting the bed after each cycle, fractional } \\ & \text { Fraction of area occupied by the urea feed in the area defined by } \Delta D_{f w}, \\ x_{r} & \text { fractional } \\ Y & 1-x_{e}, \text { fractional } \\ z & \text { Defined in Equation (2.11) } \\ z_{c} & \text { Axial destance from base of the bed, } \mathrm{m} \\ & \text { Length of spray zone from base of the bed, } \mathrm{m} \\ & \text { Location of particle's entry into the spout for urea feed particles, } \mathrm{m}\end{array}$

Greek Letters

$\alpha_{p} \quad$ Defined in Equation (2.8)

$\alpha, \beta, \varepsilon, \gamma \quad$ Exponent in Equation (2.3)

$\beta_{p} \quad$ Particle-fluid interaction term, dimensionless 
$\Delta \quad$ Difference

$\Delta D_{f} \quad$ Twice the width of annulus covered by feed adjacent to the wall of the spouted bed at the top of the bed, $m$

$\varepsilon$

Voidage, fractional

$\Phi\{z\} \quad$ Probability density, $\mathrm{m}^{-1}$

$\phi$

Spray angle, ${ }^{\circ}$

$\phi_{s}$

Particle shape factor or sphericity, fractional

$\eta$

Collection efficiency, fractional

$\varphi$

Defined in equation (2.14), dimensionless

$\mu$

Fluid viscosity, $\mathrm{kg} \cdot \mathrm{m} / \mathrm{s}$

$\mu$

Viscosity of liquid spray, $\mathrm{kg} \cdot \mathrm{m} / \mathrm{s}$

$\theta$

Included spouted bed cone angle, ${ }^{\circ}$

$\rho$

Fluid density, $\mathrm{kg} / \mathrm{m}^{3}$

$\rho_{a}$

Atomizing air density, $\mathrm{kg} / \mathrm{m}^{3}$

$\rho_{b}$

Bulk density of loose-packed solids, $\mathrm{kg} / \mathrm{m}^{3}$

$\rho_{c}$

Aerosol density, $\mathrm{kg} / \mathrm{m}^{3}$

$\rho_{l}$

Density of liquid spray, $\mathrm{kg} / \mathrm{m}^{3}$

$\rho_{p}$

Particle density, $\mathrm{kg} / \mathrm{m}^{3}$

$\rho_{u}$

Density urea particles, $\mathrm{kg} / \mathrm{m}^{3}$

$\Sigma$

Sum

$\sigma$

Standard deviation (Table 5.12), fractional

$\sigma_{g}$

Log-standard geometric deviation, $m$

$\sigma_{l}$

Surface tension of liquid spray, dyn/cm

$\psi$

Stream function, dimensionless

$\nabla$

Directional gradient 


\section{Subscripts}

$\begin{array}{ll}o & \text { Condition at } z=0 \\ a & \text { per cycle } \\ a s & \text { Annulus; atomizing air } \\ H & \text { Atomizing air in spout } \\ i & \text { Condition at } z=\mathbf{H} \\ l & \text { Pertaining to an individual particle or 'cut' } \\ m a & \text { Liquid spray } \\ m f & \text { At minimum atomization } \\ m p & \text { At minimum fluidization } \\ m s & \text { At minimum pulsation } \\ p & \text { At minimum spouting } \\ s & \text { Bed particle } \\ k & \text { Spout; sulfur }\end{array}$




\section{References}

Abramovich, G.N., The Theory of Turbulent Jets, M.I.T Press, Cambridge, 1963.

Allen, S.E., "Sulfur-coated urea: cover controls nitrogen release", Trees and Turf Mag., June 1980, p54.

Allen, S.E., Terman, G.L. and Hunt, C.M., "Soluable and slow-release nitrogen fertilizer effects on grass forage as influenced by rate and placement", J. Agric. Sci. Can., v77, p397, 1971.

American Society of Mechanical Engineers, Fluid Meters: Their Theory and Application, (6th edition; Edited by H.S. Bean), The American Society of Mechanical Engineers, New York, 1971.

Amirshahidi, M.S., Ph.D. Thesis, Lehigh Univ., 1984.

Balasubramanian, M., Meisen, A. and Mathur, K.B., "Spouted bed collection of solid aerosols in the presence of electrical effects", CJChE, v 56, p297, 1978.

Barton, R.K., Rigby, G.R. and Ratcliffe, J.S., "Fluid-solids contacting in spouted beds", Mech. Chem. Eng. Trans., v4, p105, 1968.

Becker, H.A. and Sallans, H.R., "On the continuous, moisture diffusion controlled drying of solid particles in a well mixed, isothermal bed", Chem. Eng. Sci., v13, p97, 1961.

Behie, S.W., Beekmans, J.M., Knetting, P. and Bulani, W., "Aerosol particle collection in a vertical pneumatic transport line", CJChE, v50, p241, 1972.

Behie, S.W. and Beekmans, J.M., "On the efficiency of a venturi scrubber", CJChE, v51, p430, 1973.

Berruti, R., Rovero, G. and Piccinini, N., "A coating process in a spouted bed: a mathematical model", Ing. Chim. Ital., v20, No. 7-8, p51, 1984.

Bitron, M.D., "Atomization of liquids by supersonic air jets", Ind. Eng. Chem., v47, p23, 1955.

Blouin, G.M., Rindt, D.W. and Moore, O.E., "Sulfur-coated fertilizers for controlled release: Pilot plant production", J. Agr. Food. Chem., v19, p801, 1971. 
Bowen, I.G. and Davis, G.P., "Particle size distribution and the estimation of Sauter mean diameter", Shell Technical Report No. ICT/28, October, 1951.

Box, G.E., Hunter, W.G. and Hunter, J.S., Statistics for Experimenters, Wiley and Sons, New York, 1978.

Bridgewater, J. and Mathur, K.B., "Prediction of spout diameter in a spouted bed - A theoretical model", Powder Tech., v6, p183, 1972.

Brown, G.W., Modern Mathematics For Engineers, 1956 (Edited by Beckenback, E.F.), chapter 12, McGraw-Hill, New York, 1956.

Chaterjee, A., "Effect of particle diameter and apparent particle density on internal solid circulation rate in air-spouted beds", Ind. Eng. Chem. PDD., v9, p531, 1970.

Clift, R., Ghadiri, M. and Thambimuthu, K.V., "Filtration of gases in fluidised beds", Progress and Filtration and Separation, v2, (Edited by Wakeman, R.J.), Elsevier, Amsterdam, p75, 1981.

Clift, R., Grace, J.R. and Weber, M.E., Bubbles, Drops and Particles, Academic Press, New York, 1978.

Conroy, E.H. and Jonstone, H.F., "Combustion of sulfur in a venturi spray burner", Ind. Eng. Chem., v41, p 2741, 1949.

Cook, H.H. and Bridgewater, J., "Segregation in spouted beds", CJChE, v56, p636, 1978.

Cox, D.R., Renewal Theory, Methuen and Co. Ltd., London, pp91-101, 1967.

Dale, J.M. and Ludwig, A.C., Mechanical Properties of Sulfur Allotropes, Mat. Res. and Stand., 1965.

Davies, C.N., Aerosol Science, Academic Press, London, 1966.

Davis, L.H., "Two grass field trials with sulfur coated urea to examine its potential as a slow release nitrogen fertilizer in the UK", J. Sci. Food Agr., v24, p63, 1973.

Day, J.Y., Morgan, M.H., III and Littman, H., "Measurements of Spout Voidage Distributions, Particle Velocities and Particle Circulation Rates in Spouted Beds of Coarse Particles - II. Experimental Verification", Chem. Eng. Sci., v42, no.6, p1461, 1987.

Donahue, J. and Meyer, B., "The naming of sulphur allotropes", Elemental Sulphur (Edited by Meyer, B.), John Wiley and Sons, New York, 1965. 
Epstein, N. and Grace, J.R., Handbook of Powder Science and Technology, 1984 (Edited by Otten, L. and Fayed, M.E.), chapter 11, Van Nostrand Reinhold, New York, 1984.

Epstein, N. and Levine, S., "Non-Darcy Flow and Pressure Distrubution in a Spouted Bed", in Fluidization, Proc. 2nd Engng. Found. Conf., Cambridge, 1978 (Edited by Davidson, J.F. and Keairns D.L.), p98, Cambridge University Press, 1978.

Epstein, N., Lim, C.J. and Mathur, K.B., "Data and models for flow distribution and pressure drop in spouted beds", CJChE, v56, p436, 1978.

Ergun, S., "Flow through packed columns", Chem. Eng. Prog., v48, p89, 1952.

Freeport Sulphur Company (Edited by Tuller, W.N.), The Sulphur Data Book, McGrawHill, New York, 1954.

Gerald, C.F. and Wheatley, P.O., Applied Numerical Analysis, Addison-Wesley, Reading, 1984.

Gnyp, A.W., St. Pierre, C.C., Smith, D.S., Mozzon, D. and Steiner, J., "S-type pitot tube coefficients: An experimental investigation of the effect of pitot tube-sampling probe configurations on the magnitude of the S-type pitot tube coefficients for commercially available source sampling probes", (written for Ontario Ministry of the Environment), University of Winsor, 1975.

Grace, J.R., "Contacting modes and behaviour classification of gas-solid and other twophase suspensions", CJChE, v64, p353, 1986.

Grace, J.R., "Fluidized bed hydrodynamics", Chapter 8.1 in Handbook of Multiphase Systems, ed. G. Hetsroni, Hemisphere, Washington, 1982.

Grace, J.R. and Mathur, K.B., "Height and structure of the fountain region above spouted beds", CJChE, v56, p533, 1978.

Harnett, D.L., Statistical Methods, 3rd ed., Addison-Wesley, Reading, 1982.

Hidy, G.M., Aerosols: An Industrial and Environmental Science, Academic Press, Orlando, 1984.

Hill, Jr., C.G., Chemical Engineering Kinetics and Reactor Design, Wiley and Sons, New York, 1977.

Jackson, R., "The mechanics of fluidized beds", Trans. Instn. Chem. Engrs., v41, p13, 1968. 
Khoe, D.F., Ph.D. Dissertation, Delf Univ., 1980.

Kim, K.Y. and Marshall, Jr., W.R., "Drop size distributions from pneumatic atomizers, AIChE J., v17, p575, 1971.

Krzywanski, R., Ph. D. Dissertation, U.B.C., 1992.

Krzywanski, R., Epstein, N and Bowen, B.; "Multi-dimensional model of a spouted bed", CJChE, v70, p858, 1992.

Kugo, M., Watanabe, N., Uemaki, O. and Shibata, T., Bul. Hokkaido Univ. Sapporo Jap., v39, p95, 1965.

Kutluoglu, E., Grace, J.R., Murchie, K.W. and Cavanagh, P.H., "Particle segregation in spouted beds", CJChE, v61, p308, 1983.

Lefroy, G.A. and Davidson, J.F., "The mechanics of spouted beds", Trans. Inst. Chem. Engrs., v47, pT120, 1969.

Lewis, H.C., Edwards, D.G., Goglia, M.J., Rise, R.I. and Smith, L.W., "Atomization of liquids in high velocity gas streams", Ind. Eng. Chem., v40, p67, 1948.

Lim, C.J., Ph.D. Dissertation, U.B.C., 1975.

Lim, C.J. and Grace, J.R., "Spouted bed hydrodynamics in a $0.91 \mathrm{~m}$ diameter vessel", CJChE, v65, p366, 1987.

Lim, C.J. and Mathur, K.B., Fluidization, "Modeling of particle movement in spouted beds", Fluidization, Proc. 2nd Engng. Found. Conf., Cambridge, 1978 (Edited by Davidson, J.F. and Keairns D.L.), Cambridge University Press, p104, 1978.

Lim, J., B.A.Sc. Thesis, U.B.C., 1978.

Littman, H., Morgan, M.H., Narayanan, P.V., Kim, S.J., Dan, J. and Lazarek, G.M., "An axisymmetric model of flow in the annulus of a spouted bed of coarse particles. Model, experimental verification and residence time distribution", CJChE, v63, p188, 1985.

Littman, H., Narayanan, P.V., Tomlins, A.H. and Friedman, M.L., "A complete theoretical model for a first order isothermal catalytic reaction in a spouted bed", AIChE Symp. Ser. No. 205, v77, p174, 1981.

Littman, H., Morgan, M.H., Vukovic, D.V., Zdanski, F.K. and Grbavcic, Z.B., "A theory for predicting the maximum spoutable height in a spouted bed", CJChE, v55, p497, 1977. 
Lunde, K.E. and Lapple, C.E., "A critique on the state of the art of dust and mist collection", Chem. Eng. Prog., v53, No. 8, p385, 1957.

Mamuro, T. and Hattori, H., "Flow pattern of fluid in spouted beds", J. Chem. Eng. Japan, v1, p1, 1968.

Mann, U., Correspondence, Ind. Eng. Chem. Proc. Des. Dev., v17, p103, 1978.

Mann, U., "Analysis of spouted bed coating and granulation. 1. Batch operation", Ind. Eng. Chem. Proc. Des. Dev., v22, p288, 1983.

Mann, U. and Crosby, E.J., "Cycle time distribution measurements in spouted beds", CJChE, v53, p579, 1975.

Mann, U., Rubinovitch, M. and Crosby, E.J., "Characterization and analysis of continuous recycle systems: Part I. Single unit", AIChE J., v25, No. 5, p873, 1979.

Mani, U. and Rubinovitch, M., "Characterization and analysis of continuous recycle systems: Part II. Cascade", AIChE J., v27, No. 5, p829, 1981.

Mann, U., Crosby, E.J. and Rubinovitch, M., "Number of cycles distribution in circulating systems", Chem. Eng. Sci., v29, p761, 1974.

Mann, U. and Crosby, E.J., "Cycle time distribution measurements in spouted beds", CJChE, v53, p579, 1975.

Mathur, K.B. and Epstein, N., Spouted Beds, Academic Press, New York, 1974.

Mathur, K.B. and Lim, C.J., "Vapour phase chemical reaction in spouted beds: a theoretical model", Chem. Eng. Sci., v29, p789, 1974.

Mathur, K.B. and Gishler, P.E., "A technique for contacting gases with coarse solid particles", AIChE J., v1, p157, 1955.

McNab, G.S., "Prediction of spout diameter", Brit. Chem. Eng. and Proc. Tech., v17, p532, 1972.

McNab, G.S. and Bridgwater, J., "Spouted beds - estimation of spouting pressure drop and the particle size for deepest bed", Proc. European Congress on Particle Technology, Nuremberg, 1977.

Meisen, A., Lee, V. and Le, V., unpublished report, 1986.

Meisen, A., Bobkowicz, A.J., Cooke, N.E. and Farkas, E.J., "The separation of micronsize particles from air by diffusiophoresis", CJChE, v49, p449, 1971. 
Meisen, A. and Mathur, K.B., "The spouted bed aerosol collector: a novel device for separating small particles from gases", Multi-Phase Flow Systems Symp., Inst. Chem. Eng. Ser. No. 38, paper K3, 1974.

Meisen, A. and Mathur, K.B., "Production of sulphur-coated urea by the spouted bed process", Paper presented at the 2nd International Conference on Fertilizers. Proceeding of the British Sulfur Corporation - Part I, December 3-6, pXIV-2, 1978.

Moore, C., UBC ODEPACK: A Systematized Collection of ODE Solvers, University of British Columbia Computing Centre, 1989.

Morgan, M.H., III and Littman, H., "General Relationships for the Minimum Spouting Pressure Drop Ratio and the Spout-annular Interfacial Condition in a Spouted Bed.", Fluidization, Proc. 3rd Engng. Found. Conf., Henniker (Edited by Grace, J.R. and Matsen, J.M.), Plenum Press, New York, p287, 1980.

Morgan, M.H., III, Day, J.Y. and Littman, H., "Spout voidage distribution, stability and particle circulation rates in spouted beds of coarse particles - I. theory", Chem. Eng. Sci., v40, No. 8, p1367, 1985.

Mugele, R.A. and Evans, H.D., "Droplet size distribution in sprays", Ind. Eng. Chem., $\mathrm{v} 43, \mathrm{p} 1318,1951$.

Murphy, C.H., Handbook of Particle Sampling and Analysis Methods, Verlag Chemie International, Deerfield Beach, Fl., 1984.

Nicol, T., UBC RANDOM: Random Number Generators, University of British Columbia Computing Centre, 1986.

Nukiyama, S. and Tanasawa, Y., "An experiment on the atomization of liquid", Trans. Soc. Mech. Engrs. (Japan), v5, p1, 1939.

Orr, C., Jr. Particulate Technology, MacMillan, New York, 1966.

Perry, R.H., Green, D.W. and Maloney, J.O., Perry's Chemical Engineering Handbook, 6th ed., McGraw-Hill, New York, 1984.

Piccinini, N., Bernhard, A., Campagna, P. and Vallana, F., "Segregation phenomena in spouted beds", CJChE, v55, p122, 1977.

Piccinini, N., Grace, J.R. and Mathur, K.B., "Vapour phase chemical reaction in spouted beds: a theoretical model", Chem. Eng. Sci., v34, p1257, 1979. 
Quinlan, M.J. and Ratcliffe, J.S., "Consequential effects of air drying wheat - spouted bed design and operation", Mech. and Chem. Eng. Trans., Australia, p19, May

Randolph, A.D. and Larson, M.P., Theory of Particular Processes, Academic Press, New York, 1971.

Richardson, J.F. and Zaki, W.N., "Sedimentation and fluidization", Trans Inst. Chem. Eng., v32, p35, 1954.

Ripley, B., Stochastic Simulation, John Wiley \& Sons, New York, 1987.

Robinson, T. and Waldie, B., "Dependency of growth on granule size in a spouted bed granulator", CJChE, v56, p632, 1978.

Romankov, P.G. and Rashkovskaya, N.B., "Drying in a Suspended State" 2nd ed., in Russian. Chem. Publ. House, Leningrad Branch, 1968.

Rovero, G., Piccinini, W., Grace, J.R., Epstein, N. and Brereton, C.M.H., "Gas flow distribution in conical-base spouted beds", Chem. Eng. Sci., v38, No. 4, p557, 1983.

Rovero, G., Piccinini, N. and Lupo, A., "Solids velocities in full and half-sectional spouted beds", Entropie, No. 124, p43, 1985.

Rubinstein, R.Y., Simulation and the Monte Carlo Method, John Wiley \& Sons, New York, 1981.

Runnals, A., An Introduction to MTS, University of British Columbia Computing Centre, 1987.

Rushton, J.H. and Oldshue, J.Y., "Mixing - present theory and practice", Chem. Eng. Prog., v49, No. 4, p161, 1953.

Sauter, J., "Determining the efficiency of atomization by its fineness and uniformity", NACA Tech. Memo., p396, 1927.

Shirley, A.R. Jr., and Meline, R.S., New Uses of Sulfur, Advances in Chemistry Series, v140, AChS, Washington, D.C., 1975.

Singiser, R.E., Heiser, A.L. and Prillig, E.B., "Air-suspension tablet coating", Chem. Eng. Prog., v 62, no. 6, p107, 1966.

Stanek, V., Szekely, "Three dimensional flow of fluids through nonumiform packed beds", AIChE J., v20, p974, 1974.

Stauffer Chemical Co., Bulletin. 
Stocker, R.K., Ph.D. Dissertation, Univ. of Calgary, 1987.

Thambimuthu, Ph. D. Dissertation, University of Cambridge, 1980.

Thorley, B., Shaunby, J.B., Mathur, K.B. and Osberg, G.L., "An analysis of air and solid flow in spouted wheat bed", CJChE, v37, p184, 1959.

Tisdale, S.L., Nelson, W.L. and Beaton, J.D., Soil Fertility and Fertilizers (4th ed.), Macmillan, New York, 1985.

Tsai, S.E., M.A.Sc. Thesis, Dept. of Chemical Engineering, U.B.C., 1986.

Uemaki, O. and Mathur, K.B., "Granulation of ammonium sulfate fertilizer in a spouted bed", Ind. Eng. Chem. Proc. Des. Dev., v15, p504, 1976.

Vaessen, W., UBC NLP: Non-linear Function Optimization, University of British Columbia Computing Centre, 1984.

Waddington, D.V. and Duich, J.M., "Two grass field trials with sulfur coated urea to examine its potential as a slow release nitrogen fertilizer in the UK", Agron. J., v68, p812, 1976.

Wan-Fyong, F., Romankov, P.G. and Rashkovskaya, N.B., "Research on the hydrodynamics of the spouting bed", Zh. Prikl. Khim., v42, p609, 1969.

Weiss, P.J., M.A.Sc. Thesis, Dept. of Chemical Engineering, U.B.C., 1981.

Weiss, P.J. and Meisen, A., "Laboratory studies on sulphur-coating urea by the spouted bed process", CJChE, v61, p440, 1983.

Wu, S.W.M., Lim, C.J. and Epstein, N., "Hydrodynamics of spouted beds at elevated temperatures", Chem. Eng. Comm., v62, p251, 1987.

Zee, C.J., B.A.Sc. Thesis, U.B.C., 1977. 


\section{Appendix l: \\ Supporting Derivations and Methodology}

In this section, selected experimental and numerical methods used to determine various model and operating variables are provided. All symbols used in this section are consistent with the symbols used in the main body of this thesis, unless specified otherwise. The following is a list of supporting materials given in this section

I-1. Determination of Shutter Area

I-2. Model II Derivation for Forced Urea Feed

I-3. Calculation Method for Vector Ergun Eqaution

I-4. Sulfur Sampling Devices

I-5. Batch Coating Model

I-6. Minimum Spouting Velocity Predictions by Wan-Fyong et al. Equation 


\section{1-1. Determination of Shutter Area}

As shown by Figure 3.4, a shutter was used to control the area of spouting air flow into the bed. The shutter consisted of five leaves which formed a circle when the shutter was fully open and formed a pentagon when fully closed. The shape of the shutter opening was an irregular shape when the shutter was not fully closed or fully opened. In this section, the area of the shutter was determined by examining the shape of the shutter for all positions.

When the shutter was fully open, the shape of the opening was close to a perfect circle. this shape was assumed to be a circle and the radius of the circle is denoted by $R$. The

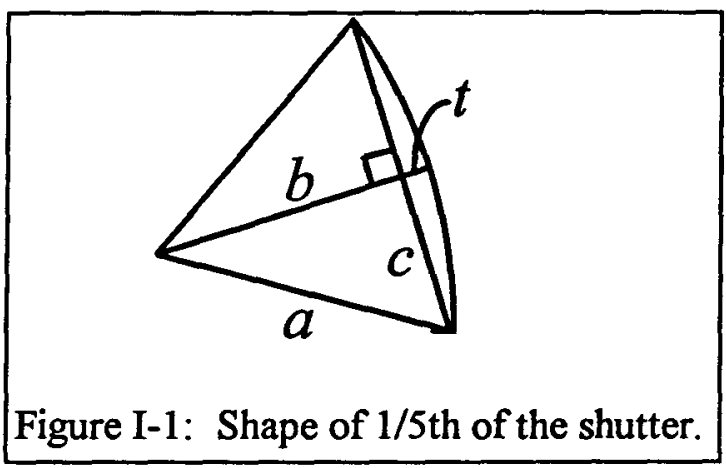
shape of the shutter was not a circle nor a pentagon when the shutter was not fully open, and the area of the shutter representing one-fifth of the area is shown in Figure I-1. The measured width of the shutter opening, $D_{e}$, is given by

$$
D_{e}=a+b+t
$$

where $a, b$ and $t$ are given in Figure I-1. The angle between lines $a$ and $b$ is equal to $36^{\circ}$; therefore,

$$
\text { and } \quad \begin{aligned}
b & =a \cos \left(36^{\circ}\right) \\
c & =\sqrt{a^{2}-b^{2}}
\end{aligned}
$$

The length of $t$ can be determined by considering a section of the fully open shutter shown in Figure I-2. The length of $t$ is given by

$$
t=R-L
$$

where $L=\sqrt{R^{2}-c^{2}}$

The angle, $\phi$, between $R$ and $L$ is then 


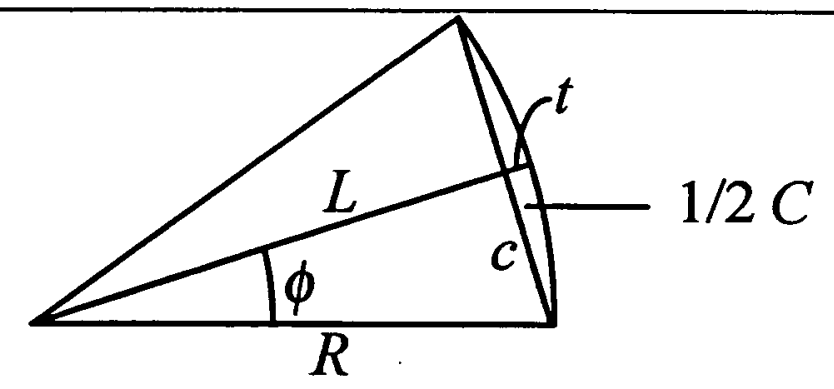

Figure I-2: Shape of a section of fully open shutter with the same base length $(t)$ shown in Figure I-

$$
\phi=\cos ^{-1}(L / R)
$$

and the area outside of the triangle is given by

$$
C=\phi R^{2}-c \cdot L
$$

By substituting Equations (I-2) and (I-4) into Equation (I-1), the value of $a$ can be determined:

$$
a=\frac{-\beta \pm \sqrt{\beta^{2}-4 \alpha \gamma}}{2 \alpha}
$$

where $\alpha=2\left(1+\cos 36^{\circ}\right)$

$$
\begin{aligned}
& \beta=2\left(R-D_{e}\right)\left(1+\cos 36^{\circ}\right) \\
& \gamma=D_{e}^{2}-2 D_{e} R
\end{aligned}
$$

Substituting $a$ into Equations (I-2) and (I-3) gives $b$ and $c$. The values of $t$ and $\phi$ can be determined from Equations (I-4) to (I-6). It follows that the area of the shutter $\left(A_{0}\right)$ shown in Figure I-1 is given by

$$
A_{o}=5(b \cdot c+C)
$$

and the orifice diameter, $d_{i}$, based on the flow area is given by

$$
d_{i}=\sqrt{A_{o} /(\pi / 4)}
$$




\section{I-2. Model II Derivation for Forced Urea Feed}

Model II derivation for the spouted bed coating unit that forces the feed into the spray zone is given in this section. The assumptions are that all feed enters the spray zone in the first cycle and that the volume of the bed particles does not change significantly. The simplified diagram of this system is shown below.

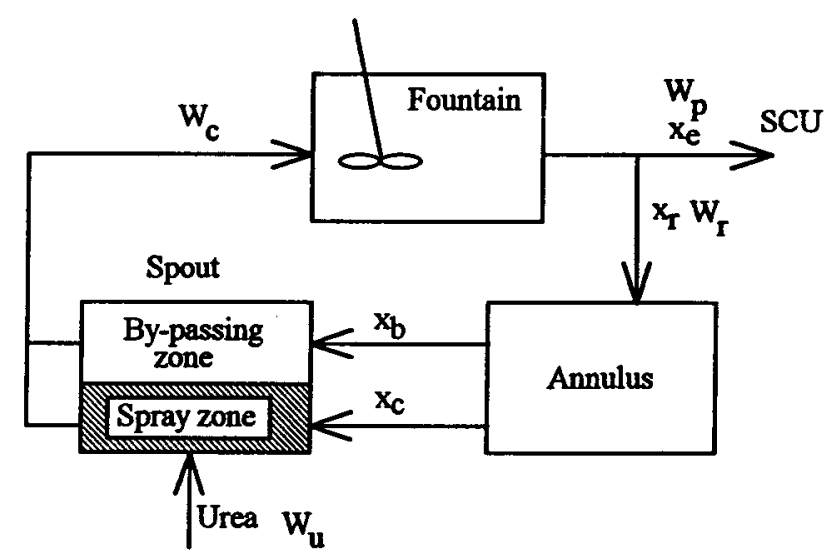

Figure I-3: Simplified flow sheet of a forced feed system.

Since the volume of the particles is assumed to be constant, the percentage of the feed that enters the spray zone is equal to that leaving the bed; therefore, the spray zone will be reduced by the same amount for the returning particles. It follows that

$$
x_{c}=\left(1-x_{c}-x_{b}^{*}\right) /\left(1-x_{c}\right)
$$

and $\quad x_{b}=x_{b}^{*} /\left(1-x_{e}\right)$

where $x_{c}$ and $x_{b}$ are probabilities of returning particles entering and by-passing the spray zone. $x_{b}^{*}$ is the absolute probability of particles by-passing the spray zone. The probability of particles not receiving any coat is zero since all particles are forced through the spray zone as it is introduced into the bed. The probability of particles receiving one or more coat is given by 


$$
\begin{aligned}
P_{1 c} & =x_{c}+x_{c} x_{b} x_{r}+x_{c} x_{b}^{2} x_{r}^{2}+x_{c} x_{b}^{3} x_{r}^{3}+\cdots \\
P_{2 c} & =x_{c} x_{c} x_{r}+2 x_{c} x_{c} x_{b}^{2} x_{r}+3 x_{c} x_{c} x_{b}^{2} x_{r}^{2}+4 x_{e} x_{b}^{3} x_{r}^{3}+\cdot \cdot \\
P_{3 c} & =x_{c} x_{c}^{2} x_{r}^{2}+3 x_{c} x_{c}^{2} x_{b}^{2} x_{r}+6 x_{c} x_{c}^{2} x_{b}^{2} x_{r}^{2}+10 x_{c} x_{c}^{2} x_{b}^{3} x_{r}^{3}+\cdot \cdot \\
& \vdots \\
P_{k c} & =\left(\begin{array}{l}
k \\
k
\end{array}\right) x_{c} x_{c}^{k-1} x_{r}^{k-1}+\left(\begin{array}{c}
k+1 \\
k
\end{array}\right) x_{c} x_{c} x_{b}^{2} x_{r}+\left(\begin{array}{c}
k+2 \\
k
\end{array}\right) x_{c} x_{c} x_{b}^{2} x_{r}^{2}+\cdots
\end{aligned}
$$

Using the infinite series representation in Table 4.2, this equation can be reduced to

$$
P_{k c}=\frac{x_{e} x_{c}^{k-1} x_{r}^{k-1}}{\left(1-x_{b} x_{r}\right)^{k}}
$$

and $\bar{m}_{s}$ using Equation (4.10) becomes

$$
\overline{m_{s}}=k \cdot m_{s 1}\left(x_{b}+x_{c} / x_{c}\right)
$$

the sulfur content distribution equation is obtained by substituting above expression into Equation (4.3), which is gives

$$
\overline{X_{s}}(k)=\frac{k \cdot x_{e} \overline{X_{s}}}{k \cdot x_{r} \bar{X}_{s}+\left(x_{b} x_{c}+x_{c}\right)\left(1-\overline{X_{s}}\right)} \text { for } k=1, \infty
$$




\section{1-3. Calculation Method for Vector Ergun Equation}

The basic derivation is identical to that given in Rovero et al. (1983). The derivation is shown here only for verification purposes.

The vector Ergun equation is given by

$$
-\nabla P_{a}=\mathbf{U}\left(f_{1}+f_{2}|\mathbf{U}|\right)
$$

Applying the curl operation to this equation gives

$$
-\nabla \times \mathbf{U}=\mathbf{U} \times \nabla \ln \left(f_{1}+f_{2}|\mathbf{U}|\right)
$$

Introducing stream functions defined for axisymmetric motion about the axis of the flow,

$$
u_{z}=-\frac{1}{r} \frac{\partial \psi}{\partial r} \text { and } u_{r}=\frac{1}{r} \frac{\partial \psi}{\partial z}
$$

Equation (I-21) can be rewritten as

$$
\begin{aligned}
& \left\{\frac{f_{1} r}{f_{2}}\left[\left(\frac{\partial \psi}{\partial z}\right)^{2}+\left(\frac{\partial \psi}{\partial r}\right)^{2}\right]^{1 / 2}+\left[2\left(\frac{\partial \psi}{\partial r}\right)^{2}+\left(\frac{\partial \psi}{\partial z}\right)^{2}\right]\right\} \frac{\partial^{2} \psi}{\partial r^{2}} \\
& +\left\{\frac{f_{1} r}{f_{2}}\left[\left(\frac{\partial \psi}{\partial z}\right)^{2}+\left(\frac{\partial \psi}{\partial r}\right)^{2}\right]^{1 / 2}+\left[2\left(\frac{\partial \psi}{\partial z}\right)^{2}+\left(\frac{\partial \psi}{\partial r}\right)^{2}\right]\right\} \frac{\partial^{2} \psi}{\partial z^{2}} \\
& -\left\{\frac{f_{1}}{f_{2}}\left[\left(\frac{\partial \psi}{\partial z}\right)^{2}+\left(\frac{\partial \psi}{\partial r}\right)^{2}\right]^{1 / 2}+\frac{2}{r}\left[\left(\frac{\partial \psi}{\partial z}\right)^{2}+\left(\frac{\partial \psi}{\partial r}\right)^{2}\right]\right\} \frac{\partial \psi}{\partial r} \\
& +2\left[\frac{\partial \psi}{\partial z} \frac{\partial \psi}{\partial r} \frac{\partial^{2} \psi}{\partial r \partial z}\right]=0
\end{aligned}
$$

Equation (I-10) was solved with the following boundary conditions:

at the wall,

$$
\psi=0
$$

at the bed surface,

$$
\partial \psi / \partial z=0
$$


and at the spout-annulus interface,

$$
\frac{\mathrm{d} P_{a}}{\mathrm{~d} z}=-\frac{1}{r} \frac{\partial \psi}{\partial r}\left\{f_{1}+\frac{f_{2}}{r}\left[\left(\frac{\partial \psi}{\partial z}\right)^{2}+\left(\frac{\partial \psi}{\partial r}\right)^{2}\right]^{1 / 2}\right\}
$$

$\mathrm{d} P / \mathrm{d} z$ was determined from the Morgan-Littman (1980) equation. Equations (I-23) to (I26) were expressed in second order finite differences forms and solved iteratively using a successive under-relaxation factor of 0.7 .

Using the calculated velocity field, the pressure profile was then determined by taking divergence of Equation ( $\mathrm{I}-20)$ and imposing the continuity condition for an incompressible fluid: i.e.,

$$
\nabla^{2} P_{a}=-f_{2}(\mathbf{U} \cdot \nabla \mathbf{U})
$$

Expansion yields

$$
\frac{1}{r} \frac{\partial}{\partial r}\left[r \frac{\partial P_{a}}{\partial r}\right]+\frac{\partial^{2} P_{a}}{\partial z^{2}}=-\frac{f_{2}}{\left(u_{r}^{2}+u_{z}^{2}\right)^{1 / 2}}\left\{u_{r}^{2} \frac{\partial u_{r}}{\partial r}+u_{r} u_{z}\left(\frac{\partial u_{z}}{\partial r}+\frac{\partial u_{r}}{\partial z}\right)+u_{z}^{2} \frac{\partial u_{z}}{\partial z}\right\}
$$

which is solved with the following boundary conditions:

at the wall

$$
\partial P_{a} / \partial \vec{n}=0
$$

at the bed surface

$$
P_{a}=P_{H}
$$

and at the spout-annulus interface, the pressure distribution given by the Morgan-Littman is applied. Second order finite difference approximations were used for all equations except at the cone wall boundary where a first order approximation was used. 


\section{1-4. Sulfur Sampling Devices}

Various sulfur sampling devices were tested for use in the spouted bed and none were found to be satisfactory. In this section, the problems associated with the devices considered for measuring the sulfur concentration in the spouted bed are summarized to aid those wishing to continue this work. Brief descriptions of each device as well as measurement procedures and problems associated with each device are provided in Tables I-1 to I-6.

All devices considered here include a tip with $1 \mathrm{~mm}$ opening. The direct sampling of sulfur (without heating the sampling tube) was not possible as molten sulfur smothered the tip of the sampler and immediately plugged the tip. Heated samplers were therefore considered. Series of tests were conducted with the packed bed heating element; however, electrical contact between the iron particles could not be established when the iron particles were in a loose state. Sintering of iron particles resulted in a very low electrical resistance across the packed bed and partial sintering resulted in a very fragile bed. Plate design and laser beam methods were also considered, but they were regarded difficult to construct and costly.

Finally, the quartz tube designs using $\mathrm{NiCr}$ wires to heat the tip were built and tested. The $3 / 8^{\prime \prime}$ three-tube was found too large to place in the spout. A smaller 1/4" two-tube was found to be a satisfactory size for sampling in the spout. However, this design also failed. The problems inherent to this design were that the quartz tube eventually broke as the tube was moved around the bed (i.e., slight force against this tube broke the tube), the exposed $\mathrm{NiCr}$ wires could not withstand the presence of urea dust and the impact of $\mathrm{SCU}$ particles, and wire sizes that can be used were limited. 
The main problem associated with hot tip samplers was that the samplers must stay red hot in order to convert $\mathrm{S}$ to $\mathrm{SO}_{2}$ upon impaction of $\mathrm{S}$. None of the samplers could accomodate enough room for insulating material. As a result, the heating tip was cooled by the air, bed materials, and sulfur spray. Unless a considerable amount of wattage can be forced into the heating elements, the element cannot stay red hot. The temperature gradient in the sampler tube caused urea to melt over the heating wires which prevented the wires from getting red hot. Due to space limitations on the sampler, the maximum wire size that can be used to heat the tip was limited to $1 / 30^{n}$, and this wire size limited the maximum heating capacity of the wires to approximately $120 \mathrm{~W}$.

In summary, an appropriate device for measuring the sulfur concentration in the spouted bed had to meet the following criteria:

- the device has to be slim enough to enter the spout without upsetting the upstream conditions,

- the device must withstand the presence of excess sulfur and urea dust at the tip,

- the tube must be strong enough to withstand the collisions to fast moving bed particles and lateral movements through the annulus,

- and the device must be large enough to accomodate electrical heating element (the heating element should not be exposed to urea or sulfur dust).

None of the devices considered in this work met all these conditions. 
Table I-1: Direct sulfur sampling.

Description of Device

Method of Sampling

Collection Mechanism

Method of Analysis

Likely Problems
$1 / 8^{\prime \prime} \mathrm{SS}$ tube connected to an in-line filter and a vacuum pump.

Vacuum suction; isokinetic sampling; length of sample timed using a stop-watch; calibrate sulfur collected as a result of introdution and withdrawl of sampler in and out of the spout

Direct sulfur sampling

Gravitational method using $\mathrm{CS}_{2}$ after urea is washed using $\mathrm{H}_{2} \mathrm{O}$; filter and parts leading to the filter are analyzed for the sulfur content

Plugging of the sampler tip due to solidification of sulfur at the tip of the sampler, and due to excess amount of sulfur near the spray nozzle; difficulty of moving the sampler into the spout using short tube (which must be inserted through the side of the wall) - longer tubes will be difficult for analysis using the gravitational method; hazards of working with $\mathrm{CS}_{2}$

Table I-2: Hot tip: packed bed $\mathrm{S} \Rightarrow \mathrm{SO}_{2}$ converter.

Description of Device

Method of Sampling

Collection Mechanism

Method of Analysis

Problems Observed
3/8" SS outer tube with alumina inner sleeve and high resistant (electrically) pellets sintered into a packed bed; the packed bed is heated to a red hot state by passing electricity through it; the tip end of the sampler serves as an electric lead and the other lead is inserted through the side of the sampler using non-electric conducting fittings

Vacuum suction; isokinetic sampling; by electrically heating the bed, $\mathrm{S}$ is converted into $\mathrm{SO}_{2}$

Heating and reacting spray deposits into $\mathrm{SO}_{2}$

Amount of $\mathrm{SO}_{2}$ determined via $\mathrm{SO}_{2}$ analyzer, and amount of $\mathrm{S}$ determined from volumetric flow rates and stoichiometry

Plugging of the tip due to difficulties in heating the tip electrically; difficulties of sintering the bed to give a certain electrical resistance - usually the resistance became too low (small voidage) or too high (little sintering, resulting in no electric contact); localized heating 
Table I-3: Hot tip: plate $\mathrm{S} \Rightarrow \mathrm{SO}_{2}$ converter.

Description of Device $3 / 8^{\text {n }} \mathrm{SS}$ outer tube with alumina inner sleeve and high resistant (electrically) plate; the plate is heated to a red hot state by passing electricity through it; two leads are inserted through the side of the sampler using non-electric conducting fittings

Method of Sampling Vacuum suction; isokinetic sampling; by electrically heating the plate, $\mathrm{S}$ is converted into $\mathrm{SO}_{2}$

Collection Mechanism Heating the spray (deposited via inertial impaction onto the plate) and converting $\mathrm{S}$ to $\mathrm{SO}_{2}$

Method of Analysis Amount of $\mathrm{SO}_{2}$ determined via $\mathrm{SO}_{2}$ analyzer, and amount of $\mathrm{S}$ determined from volumetric flow rates and stoichiometry

Likely Problems Plugging of the tip due to difficulties in heating the tip electrically; difficulties of manufacturing the plate with the electrical leads, and variable density plate

Table I-4: Hot tip: laser beam $\mathrm{S} \Rightarrow \mathrm{SO}_{2}$ converter.

\begin{tabular}{ll}
\hline Description of Device & $\begin{array}{l}3 / 8^{\prime \prime} \mathrm{mm} \text { quartz or SS tube with a beam sink (spherical ball } \\
\text { bearing) located at the end of the sampler }\end{array}$ \\
Method of Sampling & $\begin{array}{l}\text { Vacuum suction; isokinetic sampling; heat the ball with the la- } \\
\text { ser beam }\end{array}$ \\
Collection Mechanism & $\begin{array}{l}\text { Zapping the } \mathrm{S} \text { spray (deposited via inertial impaction onto the } \\
\text { ball) into } \mathrm{SO}_{2}\end{array}$ \\
Method of Analysis & $\begin{array}{l}\text { Amount of } \mathrm{SO}_{2} \text { determined via } \mathrm{SO}_{2} \text { analyzer, and amount of S } \\
\text { determined from volumetric flow rates and stoichiometry }\end{array}$ \\
Likely Problems & $\begin{array}{l}\text { Inaccurate trajectory of the laser beam due to vibrations of the } \\
\text { sampler in the bed contributed by the air and particles hitting } \\
\text { the sampler; plugging of the tip due to difficulties in heating } \\
\text { the tip electrically; incorporation of the laser beam to the exist- } \\
\text { ing apparatus due to lengthy nature of laser beam source; cost } \\
\text { and availabiltiy of laser beam }\end{array}$ \\
\hline
\end{tabular}


Table I-5: Hot tip: three tube hot wire $\mathrm{S} \Rightarrow \mathrm{SO}_{2}$ converter.

\begin{tabular}{ll}
\hline Description of Device & $\begin{array}{l}3 / 8^{\prime \prime} \text { quartz outer tube, } 1 / 4^{\prime \prime} \text { quartz inner shell and } 1 / 8 \text { " quartz } \\
\text { heating tube to hold the heating element; the tip and the inner } \\
\text { shell are heated using } \mathrm{NiCr} \text { wires; two electrical leads are } \\
\text { separated by the with the quart tubes for the inner shell and } \\
\text { outer wires are exposed to the bed }\end{array}$ \\
Method of Sampling & $\begin{array}{l}\text { Vacuum suction; isokinetic sampling; by electrically heating } \\
\text { the wire, } \mathrm{S} \text { is converted into } \mathrm{SO}_{2}\end{array}$ \\
Collection Mechanism & $\begin{array}{l}\text { Spray deposits on to the tip of the inner shell via inertial im- } \\
\text { paction onto the plate, and converts to } \mathrm{SO}_{2} \text { upon impaction }\end{array}$ \\
Method of Analysis & $\begin{array}{l}\text { Amount of } \mathrm{SO}_{2} \text { determined via } \mathrm{SO}_{2} \text { analyzer, and amount of } \mathrm{S} \\
\text { determined from volumetric flow rates and stoichiometry }\end{array}$ \\
Problems Observed & Too large to be placed in the spout
\end{tabular}

Table I-6: Hot tip: two tube hot wire $\mathrm{S} \Rightarrow \mathrm{SO}_{2}$ converter.

Description of Device $1 / 4^{\prime \prime}$ quartz outer shell and 1/8" quartz inner tube to hold the heating element; the tip and the inner shell are heated using $\mathrm{NiCr}$ wires; two electrical leads are separated by the with the quart tubes for the inner shell and outer wires separated physically by the strength of the wires; all wires are exposed to the material

Method of Sampling Vacuum suction; isokinetic sampling; by electrically heating the wire and therefore the inner shell, $\mathrm{S}$ is converted into $\mathrm{SO}_{2}$

Collection Mechanism Spray deposits on to the tip of the inner shell via inertial impaction onto the inner shell, and converts to $\mathrm{SO}_{2}$ upon impaction

Method of Analysis Amount of $\mathrm{SO}_{2}$ determined via $\mathrm{SO}_{2}$ analyzer, and amount of $\mathrm{S}$ determined from volumetric flow rates and stoichiometry

Problems Observed $\quad \mathrm{NiCr}$ wires cannot withstand the presence of urea, urea vapour and impaction of bed particles; melting and reaction of urea at the sides of the sampler, and eventually making its way to the heating elements and greatly lowering the temperature (the heating element could not sustain the red color) of the heating element; presence of large urea particles in the bed as result of urea agglomeration due to melted urea 


\section{I-5. Batch Coating Model}

Model IV may be manipulated to predict coating distributions of batch products. In the continuous coating operation, the simulation of a particle in the bed begins as the particle enters the bed and the simulation ends as the particle leaves the bed. The probability associated with the urea feed location determines the path of the particle during its first cycle through the bed, and the probability associated with the returning particles from the fountain determines the subsequent paths. The exit probability of the particle in the fountain determines the termination of the simulation for each particle. In the batch operation, all particles have the same residence time in the bed, and hence, the probabilities associated with the feed location and with the particles exiting no longer apply. Instead, the total residence time of the particle indicates the termination of the simulation. The coating amount a particle receives in each cycle can be calculated in the same way as a particle in the continuous operation; therefore, the only additional information required to determine the coating distribution for the batch product is the cycle time.

The cycle time for the particle is calculated from Equation (4.52) and the total residence time from Equation (4.55). The simulation ends when the total cycle time is equal to the total operating time.

A correction to the above equation is necessary for the first cycle. In the first cycle or at the start of a coating operation, bed particles could be located anywhere in the bed. To determine the location of each particle, the Monte Carlo method is applied. First, the vertical position of the particle in the annulus is determined using a random number $\left(N_{R}\right)$ :

$$
\begin{aligned}
& N_{R}=\left(V_{t c 0}-A_{s} h_{i}\right) / V_{t o t}, \text { for conical section } \\
& N_{R}=\left(V_{c}+\left(h_{i}-h_{c}\right) A-A_{s} h_{i}\right) / V_{t o t}, \text { for cylindrical section }
\end{aligned}
$$

where $V_{t c o}=$ bed volume in the conical section; function of $h_{i}$

$A_{s}=$ area of the spout 


$$
\begin{aligned}
& V_{\text {tot }}=\text { total bed volume } \\
& V_{c}=\text { total bed volume in conical section } \\
& A=\text { bed surface area in the cylindrical section } \\
& h_{c}=\text { cone height }
\end{aligned}
$$

The radial position $\left(D_{i}\right)$ at $h_{i}$ is determined using another random number,

$$
N_{R}=\left(D_{i}^{2}-D_{s}^{2}\right) /\left(D^{2}\left\{h_{i}\right\}-D_{s}^{2}\right)
$$

where $D\{z\}=$ bed diameter at $h_{i}$

$$
D_{s}=\text { spout diameter }
$$

The location where the particle enters the spout $\left(h_{s}\right)$ can be calculated from the following particle mass balance which assumes no radial velocity gradient in the annulus:

$$
\left.v_{a}\left(1-\varepsilon_{a}\right) A_{a}\right|_{h_{t}}=\left.v_{s}\left(1-\varepsilon_{s}\right) A_{s}\right|_{z_{s}}
$$

where $v_{a}=$ particle velocity in the annulus at $h_{i}$

$A_{a}=$ annulus area at $h_{i}$

$\varepsilon_{a}, \varepsilon_{s}=$ voidages in the annulus and the spout, respectively

$v_{s}=$ particle velocity in the spout at $z_{s}$

The value of $z_{s}$ can be determined from a root finding method. The values $v_{a}$ and $A_{a}$ are given by

$$
v_{a}=\left.\left(1-\varepsilon_{s}\right) v_{s} A_{s}\right|_{h_{i}} /\left.\left(1-\varepsilon_{a}\right) A_{a}\right|_{h_{i}}
$$

and $A_{a}=\frac{\pi}{4}\left(D_{b}+2 h_{i} \tan 30^{\circ}\right)$, for conical section

$A_{a}=\frac{\pi}{4} D^{2}$, for cylindrical section

where $D_{b}=$ bed diameter at the base of the bed

$D=$ bed diameter of the cylindrical section of the bed

The cycle time can then be calculated from Equation (4.52) with $h_{i}$ and $z_{s}$ as the limits. For subsequent cycles, the cycle time can be calculated using the same equation with the limits of $H$ and $z_{s}$. 


\section{I-6. Minimum Spouting Velocity Predictions by Wan-Fyong et al. Equation}

The Wan-Fyong et al. (1969) equation was correlated using their conical (plus short cylinder) bed data obtained under the following operating conditions: cone angle $=10-70^{\circ} ; d_{i}$ $=26-76 \mathrm{~mm} ; H=70-300 \mathrm{~mm} ; d_{p}=0.35-4 \mathrm{~mm} ; \rho_{s}=450-1390 \mathrm{~kg} / \mathrm{m}^{3} ; \rho_{b}=200-790$ $\mathrm{kg} / \mathrm{m}^{3}$. For $60^{\circ}$ conical bed, their equation becomes

$$
\left(U_{i}\right)_{m s}=0.748 U_{t}\left(H / d_{i}\right)^{0.82}
$$

where $U_{i}=4 Q_{m s} / \pi d_{i}^{2}$ and $U_{t}$ is the average terminal velocity of bed particles. The agreement between this equation and the experimental data for the conical bed was good using the corrected $d_{i}^{\prime}$ (see Figure I-4); however, it suggests $H^{0.82}$ and $d_{i}^{1.18}$ relationship with $Q_{m s}$ (in this work $H^{0.96}$ and $d_{i}^{0.37}$ relationship was found with $Q_{m s}$ ).

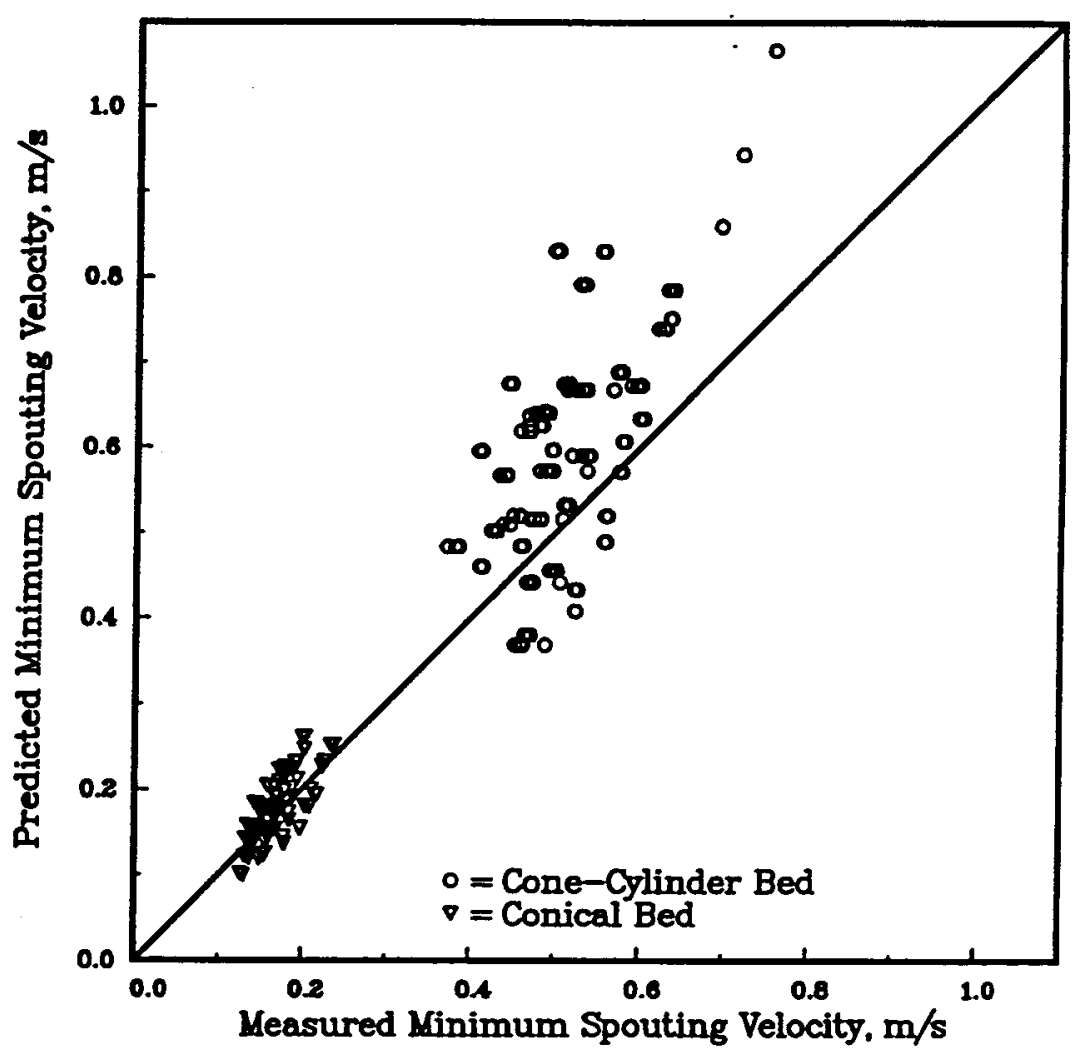

Figure I-4: Minimum spouting velocity predictions using Wan-Fyong et al. (1969) equation. 


\section{Appendix II: \\ Experimental Data and Calculated Results}

This section includes measured data and calculated results that are too lengthy to be in the main body of this thesis. All data are converted to SI units and tabulated in the following order:

Table II-1: Minimum spouting velocity data.

Table II-2: Spray drop size distribution.

Table II-3: Measured sulfur content of individual particles for selected continuous runs.

Table II-4: Predicted sulfur content of individual particles for selected continuous runs.

Table II-5: Measured sulfur content of individual particles for batch nuns shown in

Figures 4.3 and 5.25 . 
Table II-1: Minimum spouting velocity data.

\begin{tabular}{cccccccc}
$d_{\boldsymbol{p}}, \mathrm{mm}$ & $\rho_{\boldsymbol{p}}, \mathrm{kg} / \mathrm{m}^{3}$ & $d_{\boldsymbol{i}}, \mathrm{mm}$ & $Q_{\boldsymbol{a}}, \mathrm{L} / \mathrm{s}$ & $Q_{\boldsymbol{s}}, \mathrm{L} / \mathrm{s}$ & $H, \mathrm{~m}$ & $D, \mathrm{~m}$ & $T,{ }^{\circ} \mathrm{C}$ \\
\hline 2.33 & 1471 & 25.1 & 0.000 & 20.8 & 0.255 & 0.24 & $\mathbf{1 8}$ \\
2.33 & 1471 & 25.1 & 0.447 & 20.5 & 0.255 & 0.24 & 18 \\
2.33 & 1471 & 25.1 & 0.878 & 20.1 & 0.255 & 0.24 & 18 \\
2.33 & 1471 & 29.9 & 0.000 & 22.5 & 0.255 & 0.24 & 18 \\
2.33 & 1471 & 29.9 & 0.447 & 22.1 & 0.255 & 0.24 & 18 \\
2.33 & 1471 & 29.9 & 0.878 & 21.6 & 0.255 & 0.24 & 18 \\
2.33 & 1471 & 29.9 & 0.447 & 21.3 & 0.255 & 0.24 & 55 \\
2.33 & 1471 & 29.9 & 0.878 & 20.3 & 0.255 & 0.24 & 55 \\
2.33 & 1471 & 29.9 & 0.000 & 22.0 & 0.255 & 0.24 & 55 \\
2.33 & 1471 & 25.1 & 0.447 & 19.7 & 0.255 & 0.24 & 58 \\
2.33 & 1471 & 25.1 & 0.878 & 18.9 & 0.255 & 0.24 & 58 \\
2.33 & 1471 & 25.1 & 0.000 & 20.2 & 0.255 & 0.24 & 58 \\
2.33 & 1471 & 29.9 & 0.447 & 21.3 & 0.255 & 0.24 & 71 \\
2.33 & 1471 & 29.9 & 0.878 & 20.3 & 0.255 & 0.24 & 71 \\
2.33 & 1471 & 29.9 & 0.000 & 21.6 & 0.255 & 0.24 & 71 \\
2.33 & 1471 & 29.9 & 0.447 & 24.7 & 0.360 & 0.24 & 55 \\
2.33 & 1471 & 29.9 & 0.878 & 24.1 & 0.360 & 0.24 & 55 \\
2.33 & 1471 & 29.9 & 0.000 & 25.2 & 0.360 & 0.24 & 55 \\
2.33 & 1471 & 25.1 & 0.447 & 22.9 & 0.360 & 0.24 & 55 \\
2.33 & 1471 & 25.1 & 0.878 & 22.1 & 0.360 & 0.24 & 55 \\
2.33 & 1471 & 25.1 & 0.000 & 23.2 & 0.360 & 0.24 & 55 \\
2.36 & 1490 & 29.9 & 0.447 & 21.7 & 0.255 & 0.24 & 62 \\
2.36 & 1490 & 29.9 & 0.878 & 20.6 & 0.255 & 0.24 & 62 \\
2.36 & 1490 & 29.9 & 0.000 & 22.3 & 0.255 & 0.24 & 62 \\
2.36 & 1490 & 25.1 & 0.447 & 20.3 & 0.255 & 0.24 & 62 \\
2.36 & 1490 & 25.1 & 0.878 & 19.5 & 0.255 & 0.24 & 62 \\
2.36 & 1490 & 25.1 & 0.000 & 20.8 & 0.255 & 0.24 & 62 \\
2.27 & 1427 & 29.9 & 0.447 & 20.9 & 0.260 & 0.24 & 61 \\
2.27 & 1427 & 29.9 & 0.878 & 19.9 & 0.260 & 0.24 & 61 \\
2.27 & 1427 & 29.9 & 0.000 & 21.2 & 0.260 & 0.24 & 61 \\
2.27 & 1427 & 25.1 & 0.447 & 19.1 & 0.260 & 0.24 & 61 \\
2.27 & 1427 & 25.1 & 0.878 & 18.3 & 0.260 & 0.24 & 61 \\
2.27 & 1427 & 25.1 & 0.000 & 19.4 & 0.260 & 0.24 & 61 \\
2.16 & 1335 & 29.9 & 0.000 & 20.1 & 0.270 & 0.24 & 20 \\
2.16 & 1335 & 29.9 & 0.447 & 19.5 & 0.270 & 0.24 & 20 \\
2.16 & 1335 & 29.9 & 0.878 & 18.7 & 0.270 & 0.24 & 20 \\
2.16 & 1335 & 25.1 & 0.000 & 18.6 & 0.270 & 0.24 & 20 \\
2.16 & 1335 & 25.1 & 0.447 & 18.3 & 0.270 & 0.24 & 20 \\
2.16 & 1335 & 25.1 & 0.878 & 17.7 & 0.270 & 0.24 & 20 \\
& & & & & & &
\end{tabular}


Table II-1 continued.

\begin{tabular}{|c|c|c|c|c|c|c|c|}
\hline$d_{p}, \mathrm{~mm}$ & $\rho_{p}, \mathrm{~kg} / \mathrm{m}^{3}$ & $d_{i}, \mathrm{~mm}$ & $Q_{a}, \mathrm{~L} / \mathrm{s}$ & $Q_{s}, \mathrm{~L} / \mathrm{s}$ & $H, \mathrm{~m}$ & $D, \mathrm{~m}$ & $T,{ }^{\circ} \mathrm{C}$ \\
\hline 2.16 & 1335 & 29.9 & 0.000 & 23.9 & 0.405 & 0.24 & 20 \\
\hline 2.16 & 1335 & 29.9 & 0.447 & 23.6 & 0.405 & 0.24 & 20 \\
\hline 2.16 & 1335 & 29.9 & 0.878 & 23.3 & 0.405 & 0.24 & 20 \\
\hline 2.16 & 1335 & 25.1 & 0.000 & 22.0 & 0.405 & 0.24 & 20 \\
\hline 2.16 & 1335 & 25.1 & 0.447 & 21.6 & 0.405 & 0.24 & 20 \\
\hline 2.16 & 1335 & 25.1 & 0.878 & 21.2 & 0.405 & 0.24 & 20 \\
\hline 2.16 & 1335 & 29.9 & 0.000 & 18.7 & 0.270 & 0.24 & 60 \\
\hline 2.16 & 1335 & 29.9 & 0.447 & 18.3 & 0.270 & 0.24 & 60 \\
\hline 2.16 & 1335 & 29.9 & 0.878 & 17.6 & 0.270 & 0.24 & 60 \\
\hline 2.16 & 1335 & 25.1 & 0.000 & 17.5 & 0.270 & 0.24 & 60 \\
\hline 2.16 & 1335 & 25.1 & 0.447 & 16.8 & 0.270 & 0.24 & 60 \\
\hline 2.16 & 1335 & 25.1 & 0.878 & 16.0 & 0.270 & 0.24 & 60 \\
\hline 2.16 & 1335 & 29.9 & 0.000 & 22.6 & 0.405 & 0.24 & 60 \\
\hline 2.16 & 1335 & 29.9 & 0.447 & 22.3 & 0.405 & 0.24 & 60 \\
\hline 2.16 & 1335 & 29.9 & 0.878 & 21.6 & 0.405 & 0.24 & 60 \\
\hline 2.16 & 1335 & 25.1 & 0.000 & 20.2 & 0.405 & 0.24 & 60 \\
\hline 2.16 & 1335 & 25.1 & 0.447 & 19.8 & 0.405 & 0.24 & 60 \\
\hline 2.16 & 1335 & 25.1 & 0.878 & 19.2 & 0.405 & 0.24 & 60 \\
\hline 2.8 & 927 & 24.7 & 0.000 & 24.2 & 0.297 & 0.45 & 24 \\
\hline 2.8 & 927 & 28.2 & 0.000 & 25.1 & 0.297 & 0.45 & 24 \\
\hline 2.8 & 927 & 30.8 & 0.000 & 25.3 & 0.297 & 0.45 & 24 \\
\hline 2.8 & 927 & 35.1 & 0.000 & 26.9 & 0.297 & 0.45 & 24 \\
\hline 2.8 & 927 & 24.7 & 0.528 & 23.7 & 0.297 & 0.45 & 24 \\
\hline 2.8 & 927 & 28.2 & 0.528 & 24.5 & 0.297 & 0.45 & 24 \\
\hline 2.8 & 927 & 30.8 & 0.528 & 25.0 & 0.297 & 0.45 & 24 \\
\hline 2.8 & 927 & 35.1 & 0.528 & 26.5 & 0.297 & 0.45 & 24 \\
\hline 2.8 & 927 & 24.7 & 0.868 & 23.4 & 0.297 & 0.45 & 24 \\
\hline 2.8 & 927 & 28.2 & 0.868 & 24.0 & 0.297 & 0.45 & 24 \\
\hline 2.8 & 927 & 30.8 & 0.868 & 24.3 & 0.297 & 0.45 & 24 \\
\hline 2.8 & 927 & 35.1 & 0.868 & 26.0 & 0.297 & 0.45 & 24 \\
\hline 2.8 & 927 & 21.2 & 0.000 & 20.2 & 0.249 & 0.45 & 24 \\
\hline 2.8 & 927 & 24.7 & 0.000 & 21.3 & 0.249 & 0.45 & 24 \\
\hline 2.8 & 927 & 28.2 & 0.000 & 22.1 & 0.249 & 0.45 & 24 \\
\hline 2.8 & 927 & 30.8 & 0.000 & 22.7 & 0.249 & 0.45 & 24 \\
\hline 2.8 & 927 & 35.1 & 0.000 & 24.0 & 0.249 & 0.45 & 24 \\
\hline 2.8 & 927 & 21.2 & 0.528 & 19.6 & 0.249 & 0.45 & 24 \\
\hline 2.8 & 927 & 24.7 & 0.528 & 20.8 & 0.249 & 0.45 & 24 \\
\hline 2.8 & 927 & 28.2 & 0.528 & 21.8 & 0.249 & 0.45 & 24 \\
\hline 2.8 & 927 & 30.8 & 0.528 & 22.1 & 0.249 & 0.45 & 24 \\
\hline
\end{tabular}


Table II-1 continued.

\begin{tabular}{|c|c|c|c|c|c|c|c|}
\hline$d_{p}, \mathrm{~mm}$ & $\rho_{p}, \mathrm{~kg} / \mathrm{m}^{3}$ & $d_{i}, \mathrm{~mm}$ & $Q_{a}, \mathrm{~L} / \mathbf{s}$ & $Q_{s}, \mathrm{~L} / \mathrm{s}$ & $H, \mathrm{~m}$ & $D, \mathbf{m}$ & $T,{ }^{\circ} \mathrm{C}$ \\
\hline 2.8 & 927 & 35.1 & 0.528 & 23.5 & 0.249 & 0.45 & 24 \\
\hline 2.8 & 927 & 21.2 & 0.868 & 19.4 & 0.249 & 0.45 & 24 \\
\hline 2.8 & 927 & 24.7 & 0.868 & 20.3 & 0.249 & 0.45 & 24 \\
\hline 2.8 & 927 & 28.2 & 0.868 & 21.2 & 0.249 & 0.45 & 24 \\
\hline 2.8 & 927 & 30.8 & 0.868 & 21.5 & 0.249 & 0.45 & 24 \\
\hline 2.8 & 927 & 35.1 & 0.868 & 22.5 & 0.249 & 0.45 & 24 \\
\hline 2.8 & 927 & 21.2 & 0.000 & 23.6 & 0.304 & 0.45 & 60 \\
\hline 2.8 & 927 & 24.7 & 0.000 & 24.9 & 0.304 & 0.45 & 60 \\
\hline 2.8 & 927 & 28.2 & 0.000 & 25.6 & 0.304 & 0.45 & 60 \\
\hline 2.8 & 927 & 30.8 & 0.000 & 26.0 & 0.304 & 0.45 & 60 \\
\hline 2.8 & 927 & 35.1 & 0.000 & 27.6 & 0.304 & 0.45 & 60 \\
\hline 2.8 & 927 & 21.2 & 0.528 & 23.5 & 0.304 & 0.45 & 60 \\
\hline 2.8 & 927 & 24.7 & 0.528 & 24.5 & 0.304 & 0.45 & 60 \\
\hline 2.8 & 927 & 28.2 & 0.528 & 25.2 & 0.304 & 0.45 & 60 \\
\hline 2.8 & 927 & 30.8 & 0.528 & 25.8 & 0.304 & 0.45 & 60 \\
\hline 2.8 & 927 & 35.1 & 0.528 & 26.9 & 0.304 & 0.45 & 60 \\
\hline 2.8 & 927 & 21.2 & 0.868 & 23.1 & 0.304 & 0.45 & 60 \\
\hline 2.8 & 927 & 24.7 & 0.868 & 24.2 & 0.304 & 0.45 & 60 \\
\hline 2.8 & 927 & 28.2 & 0.868 & 24.8 & 0.304 & 0.45 & 60 \\
\hline 2.8 & 927 & 30.8 & 0.868 & 25.2 & 0.304 & 0.45 & 60 \\
\hline 2.8 & 927 & 35.1 & 0.868 & 26.0 & 0.304 & 0.45 & 60 \\
\hline 2.8 & 927 & 21.2 & 0.000 & 19.6 & 0.241 & 0.45 & 60 \\
\hline 2.8 & 927 & 24.7 & 0.000 & 20.5 & 0.241 & 0.45 & 60 \\
\hline 2.8 & 927 & 28.2 & 0.000 & 21.1 & 0.241 & 0.45 & 60 \\
\hline 2.8 & 927 & 30.8 & 0.000 & 21.7 & 0.241 & 0.45 & 60 \\
\hline 2.8 & 927 & 35.1 & 0.000 & 22.6 & 0.241 & 0.45 & 60 \\
\hline 2.8 & 927 & 21.2 & 0.528 & 19.3 & 0.241 & 0.45 & 60 \\
\hline 2.8 & 927 & 24.7 & 0.528 & 20.2 & 0.241 & 0.45 & 60 \\
\hline 2.8 & 927 & 28.2 & 0.528 & 20.6 & 0.241 & 0.45 & 60 \\
\hline 2.8 & 927 & 30.8 & 0.528 & 21.2 & 0.241 & 0.45 & 60 \\
\hline 2.8 & 927 & 35.1 & 0.528 & 22.2 & 0.241 & 0.45 & 60 \\
\hline 2.8 & 927 & 21.2 & 0.868 & 18.7 & 0.241 & 0.45 & 60 \\
\hline 2.8 & 927 & 24.7 & 0.868 & 19.5 & 0.241 & 0.45 & 60 \\
\hline 2.8 & 927 & 28.2 & 0.868 & 19.8 & 0.241 & 0.45 & 60 \\
\hline 2.8 & 927 & 30.8 & 0.868 & 20.2 & 0.241 & 0.45 & 60 \\
\hline 2.8 & 927 & 35.1 & 0.868 & 21.5 & 0.241 & 0.45 & 60 \\
\hline 2.7 & 1385 & 21.2 & 0.000 & 23.0 & 0.245 & 0.45 & 22 \\
\hline 2.7 & 1385 & 24.7 & 0.000 & 24.9 & 0.245 & 0.45 & 22 \\
\hline 2.7 & 1385 & 28.2 & 0.000 & 26.3 & 0.245 & 0.45 & 22 \\
\hline
\end{tabular}


Table II-1 continued.

\begin{tabular}{|c|c|c|c|c|c|c|c|}
\hline$d_{p}, \mathrm{~mm}$ & $\rho_{p}, \mathrm{~kg} / \mathrm{m}^{3}$ & $d_{i}, \mathrm{~mm}$ & $Q_{a}, \mathrm{~L} / \mathrm{s}$ & $Q_{S}, \mathrm{~L} / \mathrm{s}$ & $H, \mathrm{~m}$ & $D, \mathrm{~m}$ & $T,{ }^{\circ} \mathrm{C}$ \\
\hline 2.7 & 1385 & 30.8 & 0.000 & 26.6 & 0.245 & 0.45 & 22 \\
\hline 2.7 & 1385 & 35.1 & 0.000 & 28.1 & 0.245 & 0.45 & 22 \\
\hline 2.7 & 1385 & 21.2 & 0.528 & 22.7 & 0.245 & 0.45 & 22 \\
\hline 2.7 & 1385 & 24.7 & 0.528 & 24.4 & 0.245 & 0.45 & 22 \\
\hline 2.7 & 1385 & 28.2 & 0.528 & 25.8 & 0.245 & 0.45 & 22 \\
\hline 2.7 & 1385 & 30.8 & 0.528 & 26.3 & 0.245 & 0.45 & 22 \\
\hline 2.7 & 1385 & 35.1 & 0.528 & 27.8 & 0.245 & 0.45 & 22 \\
\hline 2.7 & 1385 & 21.2 & 0.868 & 22.2 & 0.245 & 0.45 & 22 \\
\hline 2.7 & 1385 & 24.7 & 0.868 & 23.8 & 0.245 & 0.45 & 22 \\
\hline 2.7 & 1385 & 28.2 & 0.868 & 24.9 & 0.245 & 0.45 & 22 \\
\hline 2.7 & 1385 & 30.8 & 0.868 & 25.5 & 0.245 & 0.45 & 22 \\
\hline 2.7 & 1385 & 35.1 & 0.868 & 26.6 & 0.245 & 0.45 & 22 \\
\hline 2.7 & 1385 & 21.2 & 0.000 & 27.7 & 0.289 & 0.45 & 22 \\
\hline 2.7 & 1385 & 24.7 & 0.000 & 28.6 & 0.289 & 0.45 & 22 \\
\hline 2.7 & 1385 & 28.2 & 0.000 & 29.5 & 0.289 & 0.45 & 22 \\
\hline 2.7 & 1385 & 30.8 & 0.000 & 30.2 & 0.289 & 0.45 & 22 \\
\hline 2.7 & 1385 & 35.1 & 0.000 & 31.6 & 0.289 & 0.45 & 22 \\
\hline 2.7 & 1385 & 21.2 & 0.528 & 27.4 & 0.289 & 0.45 & 22 \\
\hline 2.7 & 1385 & 24.7 & 0.528 & 28.3 & 0.289 & 0.45 & 22 \\
\hline 2.7 & 1385 & 28.2 & 0.528 & 29.1 & 0.289 & 0.45 & 22 \\
\hline 2.7 & 1385 & 30.8 & 0.528 & 29.8 & 0.289 & 0.45 & 22 \\
\hline 2.7 & 1385 & 35.1 & 0.528 & 31.2 & 0.289 & 0.45 & 22 \\
\hline 2.7 & 1385 & 21.2 & 0.868 & 27.0 & 0.289 & 0.45 & 22 \\
\hline 2.7 & 1385 & 24.7 & 0.868 & 28.0 & 0.289 & 0.45 & 22 \\
\hline 2.7 & 1385 & 28.2 & 0.868 & 28.7 & 0.289 & 0.45 & 22 \\
\hline 2.7 & 1385 & 30.8 & 0.868 & 29.3 & 0.289 & 0.45 & 22 \\
\hline 2.7 & 1385 & 35.1 & 0.868 & 30.8 & 0.289 & 0.45 & 22 \\
\hline 2.7 & 1385 & 21.2 & 0.000 & 24.2 & 0.243 & 0.45 & 65 \\
\hline 2.7 & 1385 & 24.7 & 0.000 & 25.4 & 0.243 & 0.45 & 65 \\
\hline 2.7 & 1385 & 28.2 & 0.000 & 26.0 & 0.243 & 0.45 & 65 \\
\hline 2.7 & 1385 & 30.8 & 0.000 & 26.3 & 0.243 & 0.45 & 65 \\
\hline 2.7 & 1385 & 35.1 & 0.000 & 28.5 & 0.243 & 0.45 & 65 \\
\hline 2.7 & 1385 & 21.2 & 0.528 & 23.8 & 0.243 & 0.45 & 65 \\
\hline 2.7 & 1385 & 24.7 & 0.528 & 24.8 & 0.243 & 0.45 & 65 \\
\hline 2.7 & 1385 & 28.2 & 0.528 & 25.7 & 0.243 & 0.45 & 65 \\
\hline 2.7 & 1385 & 30.8 & 0.528 & 26.0 & 0.243 & 0.45 & 65 \\
\hline 2.7 & 1385 & 35.1 & 0.528 & 27.9 & 0.243 & 0.45 & 65 \\
\hline 2.7 & 1385 & 21.2 & 0.868 & 23.2 & 0.243 & 0.45 & 65 \\
\hline 2.7 & 1385 & 24.7 & 0.868 & 24.2 & 0.243 & 0.45 & 65 \\
\hline
\end{tabular}


Table II-1 continued.

\begin{tabular}{cccccccc}
$d_{p}, \mathrm{~mm}$ & $\rho_{p}, \mathrm{~kg} / \mathrm{m}^{3}$ & $d_{i}, \mathrm{~mm}$ & $Q_{a}, \mathrm{~L} / \mathrm{s}$ & $Q_{s}, \mathrm{~L} / \mathrm{s}$ & $H, \mathrm{~m}$ & $D, \mathrm{~m}$ & $T,{ }^{\circ} \mathrm{C}$ \\
\hline 2.7 & 1385 & 28.2 & 0.868 & 25.4 & 0.243 & 0.45 & 65 \\
2.7 & 1385 & 30.8 & 0.868 & 25.4 & 0.243 & 0.45 & 65 \\
2.7 & 1385 & 35.1 & 0.868 & 26.9 & 0.243 & 0.45 & 65 \\
2.7 & 1385 & 21.2 & 0.000 & 27.7 & 0.289 & 0.45 & 65 \\
2.7 & 1385 & 24.7 & 0.000 & 28.5 & 0.289 & 0.45 & 65 \\
2.7 & 1385 & 28.2 & 0.000 & 29.3 & 0.289 & 0.45 & 65 \\
2.7 & 1385 & 30.8 & 0.000 & 29.8 & 0.289 & 0.45 & 65 \\
2.7 & 1385 & 35.1 & 0.000 & 31.8 & 0.289 & 0.45 & 65 \\
2.7 & 1385 & 21.2 & 0.528 & 27.3 & 0.289 & 0.45 & 65 \\
2.7 & 1385 & 24.7 & 0.528 & 28.3 & 0.289 & 0.45 & 65 \\
2.7 & 1385 & 28.2 & 0.528 & 28.8 & 0.289 & 0.45 & 65 \\
2.7 & 1385 & 30.8 & 0.528 & 29.3 & 0.289 & 0.45 & 65 \\
2.7 & 1385 & 35.1 & 0.528 & 31.2 & 0.289 & 0.45 & 65 \\
2.7 & 1385 & 21.2 & 0.868 & 27.0 & 0.289 & 0.45 & 65 \\
2.7 & 1385 & 24.7 & 0.868 & 27.8 & 0.289 & 0.45 & 65 \\
2.7 & 1385 & 28.2 & 0.868 & 28.4 & 0.289 & 0.45 & 65 \\
2.7 & 1385 & 30.8 & 0.868 & 28.8 & 0.289 & 0.45 & 65 \\
2.7 & 1385 & 35.1 & 0.868 & 30.4 & 0.289 & 0.45 & 65 \\
2.7 & 1385 & 24.7 & 0.000 & 33.7 & 0.332 & 0.45 & 65 \\
2.7 & 1385 & 28.2 & 0.000 & 34.9 & 0.332 & 0.45 & 65 \\
2.7 & 1385 & 24.7 & 0.528 & 33.5 & 0.332 & 0.45 & 65 \\
2.7 & 1385 & 28.2 & 0.528 & 34.5 & 0.332 & 0.45 & 65 \\
2.7 & 1385 & 30.8 & 0.528 & 36.7 & 0.332 & 0.45 & 65 \\
2.7 & 1385 & 24.7 & 0.868 & 33.2 & 0.332 & 0.45 & 65 \\
2.7 & 1385 & 28.2 & 0.868 & 34.2 & 0.332 & 0.45 & 65 \\
2.7 & 1385 & 30.8 & 0.868 & 35.6 & 0.332 & 0.45 & 65 \\
2.3 & 1045 & 24.7 & 0.000 & 22.0 & 0.293 & 0.45 & 65 \\
2.3 & 1045 & 28.2 & 0.000 & 23.2 & 0.293 & 0.45 & 65 \\
2.3 & 1045 & 30.8 & 0.000 & 23.8 & 0.293 & 0.45 & 65 \\
2.3 & 1045 & 35.1 & 0.000 & 26.0 & 0.293 & 0.45 & 65 \\
2.3 & 1045 & 24.7 & 0.528 & 21.2 & 0.293 & 0.45 & 65 \\
2.3 & 1045 & 28.2 & 0.528 & 22.6 & 0.293 & 0.45 & 65 \\
2.3 & 1045 & 30.8 & 0.528 & 23.5 & 0.293 & 0.45 & 65 \\
2.3 & 1045 & 35.1 & 0.528 & 25.4 & 0.293 & 0.45 & 65 \\
2.3 & 1045 & 24.7 & 0.868 & 20.6 & 0.293 & 0.45 & 65 \\
2.3 & 1045 & 28.2 & 0.868 & 21.7 & 0.293 & 0.45 & 65 \\
2.3 & 1045 & 30.8 & 0.868 & 22.3 & 0.293 & 0.45 & 65 \\
2.3 & 1045 & 35.1 & 0.868 & 23.8 & 0.293 & 0.45 & 65 \\
2.3 & 1045 & 24.7 & 0.000 & 26.4 & 0.341 & 0.45 & 65 \\
& & & & & & &
\end{tabular}


Table II-1 continued.

\begin{tabular}{|c|c|c|c|c|c|c|c|}
\hline$d_{p}, \mathrm{~mm}$ & $\rho_{p}, \mathrm{~kg} / \mathrm{m}^{3}$ & $d_{i}, \mathrm{~mm}$ & $Q_{a}, \mathrm{~L} / \mathrm{s}$ & $Q_{s}, \mathrm{~L} / \mathrm{s}$ & $H, \mathrm{~m}$ & $D, \mathbf{m}$ & $T,{ }^{\circ} \mathrm{C}$ \\
\hline 2.3 & 1045 & 28.2 & 0.000 & 27.0 & 0.341 & 0.45 & 65 \\
\hline 2.3 & 1045 & 30.8 & 0.000 & 28.0 & 0.341 & 0.45 & 65 \\
\hline 2.3 & 1045 & 35.1 & 0.000 & 30.4 & 0.341 & 0.45 & 65 \\
\hline 2.3 & 1045 & 24.7 & 0.528 & 25.6 & 0.341 & 0.45 & 65 \\
\hline 2.3 & 1045 & 28.2 & 0.528 & 26.7 & 0.341 & 0.45 & 65 \\
\hline 2.3 & 1045 & 30.8 & 0.528 & 27.5 & 0.341 & 0.45 & 65 \\
\hline 2.3 & 1045 & 35.1 & 0.528 & 29.8 & 0.341 & 0.45 & 65 \\
\hline 2.3 & 1045 & 24.7 & 0.868 & 25.0 & 0.341 & 0.45 & 65 \\
\hline 2.3 & 1045 & 28.2 & 0.868 & 26.2 & 0.341 & 0.45 & 65 \\
\hline 2.3 & 1045 & 30.8 & 0.868 & 27.0 & 0.341 & 0.45 & 65 \\
\hline 2.3 & 1045 & 35.1 & 0.868 & 29.0 & 0.341 & 0.45 & 65 \\
\hline 2.3 & 1045 & 24.7 & 0.000 & 30.8 & 0.344 & 0.45 & 65 \\
\hline 2.3 & 1045 & 28.2 & 0.000 & 31.6 & 0.344 & 0.45 & 65 \\
\hline 2.3 & 1045 & 30.8 & 0.000 & 33.0 & 0.344 & 0.45 & 65 \\
\hline 2.3 & 1045 & 35.1 & 0.000 & 35.8 & 0.344 & 0.45 & 65 \\
\hline 2.3 & 1045 & 24.7 & 0.528 & 30.3 & 0.344 & 0.45 & 65 \\
\hline 2.3 & 1045 & 28.2 & 0.528 & 32.1 & 0.344 & 0.45 & 65 \\
\hline 2.3 & 1045 & 30.8 & 0.528 & 32.5 & 0.344 & 0.45 & 65 \\
\hline 2.3 & 1045 & 35.1 & 0.528 & 35.3 & 0.344 & 0.45 & 65 \\
\hline 2.3 & 1045 & 24.7 & 0.868 & 29.9 & 0.344 & 0.45 & 65 \\
\hline 2.3 & 1045 & 28.2 & 0.868 & 31.6 & 0.344 & 0.45 & 65 \\
\hline 2.3 & 1045 & 30.8 & 0.868 & 31.9 & 0.344 & 0.45 & 65 \\
\hline 2.3 & 1045 & 35.1 & 0.868 & 34.5 & 0.344 & 0.45 & 65 \\
\hline 2.7 & 1385 & 21.2 & 0.000 & 23.7 & 0.245 & 0.24 & 18 \\
\hline 2.7 & 1385 & 24.7 & 0.000 & 25.2 & 0.245 & 0.24 & 18 \\
\hline 2.7 & 1385 & 28.2 & 0.000 & 26.0 & 0.245 & 0.24 & 18 \\
\hline 2.7 & 1385 & 30.8 & 0.000 & 27.1 & 0.245 & 0.24 & 18 \\
\hline 2.7 & 1385 & 35.1 & 0.000 & 28.1 & 0.245 & 0.24 & 18 \\
\hline 2.7 & 1385 & 21.2 & 0.357 & 23.3 & 0.245 & 0.24 & 18 \\
\hline 2.7 & 1385 & 24.7 & 0.357 & 24.9 & 0.245 & 0.24 & 18 \\
\hline 2.7 & 1385 & 28.2 & 0.357 & 25.8 & 0.245 & 0.24 & 18 \\
\hline 2.7 & 1385 & 30.8 & 0.357 & 27.0 & 0.245 & 0.24 & 18 \\
\hline 2.7 & 1385 & 35.1 & 0.357 & 28.1 & 0.245 & 0.24 & 18 \\
\hline 2.7 & 1385 & 21.2 & 0.528 & 23.2 & 0.245 & 0.24 & 18 \\
\hline 2.7 & 1385 & 24.7 & 0.528 & 24.8 & 0.245 & 0.24 & 18 \\
\hline 2.7 & 1385 & 28.2 & 0.528 & 25.7 & 0.245 & 0.24 & 18 \\
\hline 2.7 & 1385 & 30.8 & 0.528 & 26.7 & 0.245 & 0.24 & 18 \\
\hline 2.7 & 1385 & 35.1 & 0.528 & 28.0 & 0.245 & 0.24 & 18 \\
\hline 2.7 & 1385 & 21.2 & 0.698 & 23.0 & 0.245 & 0.24 & 18 \\
\hline
\end{tabular}


Table II-1 continued.

\begin{tabular}{|c|c|c|c|c|c|c|c|}
\hline$d_{p}, \mathrm{~mm}$ & $\rho_{p}, \mathrm{~kg} / \mathrm{m}^{3}$ & $d_{i}, \mathrm{~mm}$ & $Q_{a}, \mathrm{~L} / \mathrm{s}$ & $Q_{s}, \mathrm{~L} / \mathrm{s}$ & $H, \mathrm{~m}$ & $D, \mathrm{~m}$ & $T,{ }^{\circ} \mathrm{C}$ \\
\hline 2.7 & 1385 & 24.7 & 0.698 & 24.6 & 0.245 & 0.24 & 18 \\
\hline 2.7 & 1385 & 28.2 & 0.698 & 25.4 & 0.245 & 0.24 & 18 \\
\hline 2.7 & 1385 & 30.8 & 0.698 & 26.5 & 0.245 & 0.24 & 18 \\
\hline 2.7 & 1385 & 35.1 & 0.698 & 27.7 & 0.245 & 0.24 & 18 \\
\hline 2.7 & 1385 & 21.2 & 0.868 & 22.8 & 0.245 & 0.24 & 18 \\
\hline 2.7 & 1385 & 24.7 & 0.868 & 24.5 & 0.245 & 0.24 & 18 \\
\hline 2.7 & 1385 & 28.2 & 0.868 & 25.3 & 0.245 & 0.24 & 18 \\
\hline 2.7 & 1385 & 30.8 & 0.868 & 26.3 & 0.245 & 0.24 & 18 \\
\hline 2.7 & 1385 & 35.1 & 0.868 & 27.5 & 0.245 & 0.24 & 18 \\
\hline 2.7 & 1385 & 21.2 & 0.000 & 23.6 & 0.245 & 0.24 & 65 \\
\hline 2.7 & 1385 & 24.7 & 0.000 & 25.3 & 0.245 & 0.24 & 65 \\
\hline 2.7 & 1385 & 28.2 & 0.000 & 26.2 & 0.245 & 0.24 & 65 \\
\hline 2.7 & 1385 & 30.8 & 0.000 & 27.1 & 0.245 & 0.24 & 65 \\
\hline 2.7 & 1385 & 35.1 & 0.000 & 28.8 & 0.245 & 0.24 & 65 \\
\hline 2.7 & 1385 & 21.2 & 0.357 & 23.5 & 0.245 & 0.24 & 65 \\
\hline 2.7 & 1385 & 24.7 & 0.357 & 25.0 & 0.245 & 0.24 & 65 \\
\hline 2.7 & 1385 & 28.2 & 0.357 & 25.9 & 0.245 & 0.24 & 65 \\
\hline 2.7 & 1385 & 30.8 & 0.357 & 26.8 & 0.245 & 0.24 & 65 \\
\hline 2.7 & 1385 & 35.1 & 0.357 & 28.5 & 0.245 & 0.24 & 65 \\
\hline 2.7 & 1385 & 21.2 & 0.528 & 23.3 & 0.245 & 0.24 & 65 \\
\hline 2.7 & 1385 & 24.7 & 0.528 & 24.9 & 0.245 & 0.24 & 65 \\
\hline 2.7 & 1385 & 28.2 & 0.528 & 25.8 & 0.245 & 0.24 & 65 \\
\hline 2.7 & 1385 & 30.8 & 0.528 & 26.7 & 0.245 & 0.24 & 65 \\
\hline 2.7 & 1385 & 35.1 & 0.528 & 28.4 & 0.245 & 0.24 & 65 \\
\hline 2.7 & 1385 & 21.2 & 0.698 & 23.1 & 0.245 & 0.24 & 65 \\
\hline 2.7 & 1385 & 24.7 & 0.698 & 24.7 & 0.245 & 0.24 & 65 \\
\hline 2.7 & 1385 & 28.2 & 0.698 & 25.6 & 0.245 & 0.24 & 65 \\
\hline 2.7 & 1385 & 30.8 & 0.698 & 26.2 & 0.245 & 0.24 & 65 \\
\hline 2.7 & 1385 & 35.1 & 0.698 & 28.0 & 0.245 & 0.24 & 65 \\
\hline 2.7 & 1385 & 21.2 & 0.868 & 22.8 & 0.245 & 0.24 & 65 \\
\hline 2.7 & 1385 & 24.7 & 0.868 & 24.4 & 0.245 & 0.24 & 65 \\
\hline 2.7 & 1385 & 28.2 & 0.868 & 25.3 & 0.245 & 0.24 & 65 \\
\hline 2.7 & 1385 & 30.8 & 0.868 & 25.8 & 0.245 & 0.24 & 65 \\
\hline 2.7 & 1385 & 35.1 & 0.868 & 27.7 & 0.245 & 0.24 & 65 \\
\hline 2.7 & 1385 & 30.8 & 0.000 & 28.7 & 0.280 & 0.24 & 65 \\
\hline 2.7 & 1385 & 30.8 & 0.528 & 28.3 & 0.280 & 0.24 & 65 \\
\hline 2.7 & 1385 & 30.8 & 0.528 & 30.9 & 0.330 & 0.24 & 65 \\
\hline 2.7 & 1385 & 30.8 & 0.528 & 32.1 & 0.370 & 0.24 & 65 \\
\hline 2.7 & 1385 & 30.8 & 0.528 & 33.7 & 0.430 & 0.24 & 65 \\
\hline
\end{tabular}


Table II-1 continued.

\begin{tabular}{|c|c|c|c|c|c|c|c|}
\hline$d_{p}, \mathrm{~mm}$ & $\rho_{p}, \mathrm{~kg} / \mathrm{m}^{3}$ & $d_{i}, \mathrm{~mm}$ & $Q_{a}, \mathrm{~L} / \mathrm{s}$ & $Q_{s}, \mathrm{~L} / \mathrm{s}$ & $H, \mathrm{~m}$ & $D, \mathrm{~m}$ & $T,{ }^{\circ} \mathrm{C}$ \\
\hline 2.8 & 927 & 21.2 & 0.000 & 21.0 & 0.265 & 0.24 & 65 \\
\hline 2.8 & 927 & 24.7 & 0.000 & 22.7 & 0.265 & 0.24 & 65 \\
\hline 2.8 & 927 & 28.2 & 0.000 & 23.3 & 0.265 & 0.24 & 65 \\
\hline 2.8 & 927 & 30.8 & 0.000 & 24.4 & 0.265 & 0.24 & 65 \\
\hline 2.8 & 927 & 35.1 & 0.000 & 26.1 & 0.265 & 0.24 & 65 \\
\hline 2.8 & 927 & 21.2 & 0.357 & 20.8 & 0.265 & 0.24 & 65 \\
\hline 2.8 & 927 & 24.7 & 0.357 & 22.4 & 0.265 & 0.24 & 65 \\
\hline 2.8 & 927 & 28.2 & 0.357 & 23.0 & 0.265 & 0.24 & 65 \\
\hline 2.8 & 927 & 30.8 & 0.357 & 24.1 & 0.265 & 0.24 & 65 \\
\hline 2.8 & 927 & 35.1 & 0.357 & 25.8 & 0.265 & 0.24 & 65 \\
\hline 2.8 & 927 & 21.2 & 0.528 & 20.6 & 0.265 & 0.24 & 65 \\
\hline 2.8 & 927 & 24.7 & 0.528 & 22.0 & 0.265 & 0.24 & 65 \\
\hline 2.8 & 927 & 28.2 & 0.528 & 22.8 & 0.265 & 0.24 & 65 \\
\hline 2.8 & 927 & 30.8 & 0.528 & 23.8 & 0.265 & 0.24 & 65 \\
\hline 2.8 & 927 & 35.1 & 0.528 & 25.6 & 0.265 & 0.24 & 65 \\
\hline 2.8 & 927 & 21.2 & 0.698 & 20.6 & 0.265 & 0.24 & 65 \\
\hline 2.8 & 927 & 24.7 & 0.698 & 21.7 & 0.265 & 0.24 & 65 \\
\hline 2.8 & 927 & 28.2 & 0.698 & 22.5 & 0.265 & 0.24 & 65 \\
\hline 2.8 & 927 & 30.8 & 0.698 & 23.3 & 0.265 & 0.24 & 65 \\
\hline 2.8 & 927 & 35.1 & 0.698 & 25.5 & 0.265 & 0.24 & 65 \\
\hline 2.8 & 927 & 21.2 & 0.868 & 20.3 & 0.265 & 0.24 & 65 \\
\hline 2.8 & 927 & 24.7 & 0.868 & 21.4 & 0.265 & 0.24 & 65 \\
\hline 2.8 & 927 & 28.2 & 0.868 & 22.2 & 0.265 & 0.24 & 65 \\
\hline 2.8 & 927 & 30.8 & 0.868 & 22.6 & 0.265 & 0.24 & 65 \\
\hline 2.8 & 927 & 35.1 & 0.868 & 25.0 & 0.265 & 0.24 & 65 \\
\hline 2.3 & 1045 & 21.2 & 0.000 & 20.8 & 0.275 & 0.24 & 65 \\
\hline 2.3 & 1045 & 24.7 & 0.000 & 21.3 & 0.275 & 0.24 & 65 \\
\hline 2.3 & 1045 & 28.2 & 0.000 & 21.8 & 0.275 & 0.24 & 65 \\
\hline 2.3 & 1045 & 30.8 & 0.000 & 22.5 & 0.275 & 0.24 & 65 \\
\hline 2.3 & 1045 & 35.1 & 0.000 & 24.3 & 0.275 & 0.24 & 65 \\
\hline 2.3 & 1045 & 21.2 & 0.357 & 20.4 & 0.275 & 0.24 & 65 \\
\hline 2.3 & 1045 & 24.7 & 0.357 & 21.1 & 0.275 & 0.24 & 65 \\
\hline 2.3 & 1045 & 28.2 & 0.357 & 21.5 & 0.275 & 0.24 & 65 \\
\hline 2.3 & 1045 & 30.8 & 0.357 & 22.0 & 0.275 & 0.24 & 65 \\
\hline 2.3 & 1045 & 35.1 & 0.357 & 23.7 & 0.275 & 0.24 & 65 \\
\hline 2.3 & 1045 & 21.2 & 0.528 & 20.4 & 0.275 & 0.24 & 65 \\
\hline 2.3 & 1045 & 24.7 & 0.528 & 21.0 & 0.275 & 0.24 & 65 \\
\hline 2.3 & 1045 & 28.2 & 0.528 & 21.3 & 0.275 & 0.24 & 65 \\
\hline 2.3 & 1045 & 30.8 & 0.528 & 21.7 & 0.275 & 0.24 & 65 \\
\hline
\end{tabular}


Table II-1 continued.

\begin{tabular}{cccccccc}
$d_{p}, \mathrm{~mm}$ & $\rho_{p}, \mathrm{~kg} / \mathrm{m}^{3}$ & $d_{i}, \mathrm{~mm}$ & $Q_{a}, \mathrm{~L} / \mathrm{s}$ & $Q_{s}, \mathrm{~L} / \mathrm{s}$ & $H, \mathrm{~m}$ & $D, \mathrm{~m}$ & $T,{ }^{\circ} \mathrm{C}$ \\
\hline 2.3 & 1045 & 35.1 & 0.528 & 23.3 & 0.275 & 0.24 & 65 \\
2.3 & 1045 & 21.2 & 0.698 & 20.1 & 0.275 & 0.24 & 65 \\
2.3 & 1045 & 24.7 & 0.698 & 20.7 & 0.275 & 0.24 & 65 \\
2.3 & 1045 & 28.2 & 0.698 & 20.8 & 0.275 & 0.24 & 65 \\
2.3 & 1045 & 30.8 & 0.698 & 21.4 & 0.275 & 0.24 & 65 \\
2.3 & 1045 & 35.1 & 0.698 & 22.8 & 0.275 & 0.24 & 65 \\
2.3 & 1045 & 21.2 & 0.868 & 19.6 & 0.275 & 0.24 & 65 \\
2.3 & 1045 & 24.7 & 0.868 & 20.2 & 0.275 & 0.24 & 65 \\
2.3 & 1045 & 28.2 & 0.868 & 20.4 & 0.275 & 0.24 & 65 \\
2.3 & 1045 & 30.8 & 0.868 & 21.0 & 0.275 & 0.24 & 65 \\
2.3 & 1045 & 35.1 & 0.868 & 22.4 & 0.275 & 0.24 & 65 \\
2.3 & 1045 & 21.2 & 0.528 & 21.6 & 0.275 & 0.24 & 65 \\
2.3 & 1045 & 24.7 & 0.528 & 22.4 & 0.275 & 0.24 & 65 \\
2.3 & 1045 & 28.2 & 0.528 & 22.5 & 0.275 & 0.24 & 65 \\
2.3 & 1045 & 30.8 & 0.528 & 23.8 & 0.275 & 0.24 & 65 \\
2.3 & 1045 & 35.1 & 0.528 & 25.2 & 0.275 & 0.24 & 65
\end{tabular}


Table II-2: Spray drop size distribution.

\begin{tabular}{llllllllllll}
$d_{s} \backslash \mathrm{Run} \#$ & $1 \mathrm{a}$ & $1 \mathrm{~b}$ & $1 \mathrm{c}$ & $2 \mathrm{a}$ & $2 \mathrm{~b}$ & 3 & 4 & 5 & 6 & 7 & 8 \\
\hline 293 & 7 & 2 & 0 & 7 & 0 & 5 & 0 & 7 & 0 & 1 & 0
\end{tabular}

$\begin{array}{llllllllllll}2.93 & 7 & 2 & 0 & 7 & 0 & 5 & 0 & 7 & 0 & 1 & 0\end{array}$

$\begin{array}{llllllllllll}8.79 & 15 & 3 & 16 & 41 & 16 & 57 & 62 & 21 & 29 & 12 & 8\end{array}$

$\begin{array}{llllllllllll}14.65 & 60 & 20 & 41 & 69 & 40 & 76 & 40 & 20 & 23 & 20 & 40\end{array}$

$\begin{array}{llllllllllll}20.51 & 58 & 32 & 17 & 50 & 37 & 46 & 33 & 12 & 7 & 10 & 22\end{array}$

$\begin{array}{llllllllllll}26.37 & 48 & 10 & 9 & 21 & 11 & 23 & 19 & 6 & 4 & 3 & 41\end{array}$

$\begin{array}{llllllllllll}32.23 & 18 & 6 & 7 & 12 & 7 & 11 & 11 & 5 & 3 & 2 & 34\end{array}$

$\begin{array}{llllllllllll}38.09 & 13 & 3 & 11 & 5 & 11 & 4 & 13 & 1 & 3 & 3 & 29\end{array}$

$\begin{array}{llllllllllll}43.95 & 6 & 4 & 5 & 5 & 4 & 4 & 14 & 5 & 2 & 0 & 30\end{array}$

$\begin{array}{llllllllllll}49.8 & 4 & 4 & 2 & 1 & 3 & 5 & 11 & 4 & 0 & 2 & 16\end{array}$

$\begin{array}{llllllllllll}55.66 & 3 & 5 & 5 & 2 & 3 & 2 & 3 & 1 & 0 & 1 & 17\end{array}$

$\begin{array}{llllllllllll}61.52 & 3 & 2 & 2 & 0 & 4 & 4 & 4 & 1 & 0 & 0 & 12\end{array}$

$\begin{array}{llllllllllll}67.38 & 2 & 2 & 2 & 2 & 1 & 1 & 8 & 1 & 0 & 1 & 7\end{array}$

$\begin{array}{llllllllllll}73.24 & 2 & 1 & 1 & 1 & 0 & 2 & 0 & 0 & 0 & 1 & 5\end{array}$

$\begin{array}{llllllllllll}79.1 & 0 & 1 & 0 & 0 & 0 & 2 & 3 & 1 & 0 & 0 & 7\end{array}$

$\begin{array}{llllllllllll}84.96 & 3 & 1 & 0 & 1 & 0 & 3 & 1 & 0 & 0 & 1 & 2\end{array}$

$\begin{array}{llllllllllll}90.82 & 2 & 0 & 1 & 0 & 0 & 2 & 2 & 0 & 0 & 0 & 3\end{array}$

$\begin{array}{llllllllllll}96.68 & 2 & 2 & 1 & 0 & 1 & 0 & 0 & 0 & 0 & 0 & 2\end{array}$

$\begin{array}{llllllllllll}102.54 & 2 & 0 & 0 & 1 & 0 & 1 & 1 & 0 & 0 & 0 & 1\end{array}$

$\begin{array}{llllllllllll}108.4 & 1 & 0 & 0 & 1 & 0 & 2 & 1 & 0 & 0 & 1 & 1\end{array}$

$\begin{array}{llllllllllll}114.26 & 1 & 1 & 0 & 1 & 1 & 0 & 1 & 0 & 1 & 0 & 0\end{array}$

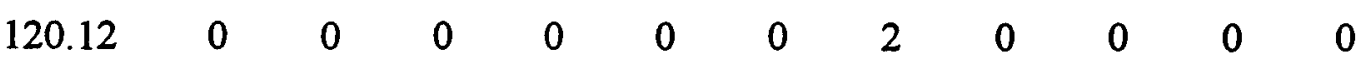

$\begin{array}{llllllllllll}125.98 & 1 & 1 & 0 & 1 & 0 & 0 & 0 & 0 & 0 & 0 & 3\end{array}$

$\begin{array}{llllllllllll}131.84 & 0 & 0 & 0 & 0 & 0 & 0 & 0 & 0 & 0 & 0 & 1\end{array}$

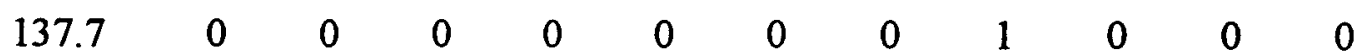
$\begin{array}{llllllllllll}143.55 & 0 & 0 & 1 & 0 & 0 & 0 & 0 & 0 & 0 & 0 & 2\end{array}$

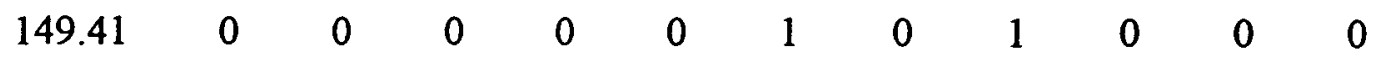
$\begin{array}{llllllllllll}>150 & 3 & 1 & 2 & 2 & 1 & 0 & 0 & 0 & 1 & 0 & 4\end{array}$

$\begin{array}{llllllllllll}\text { Total (N) } & 254 & 101 & 123 & 223 & 140 & 251 & 229 & 87 & 73 & 58 & 287\end{array}$ 
Table II-2 continued.

\begin{tabular}{cccccccccccc}
$d_{\boldsymbol{S}} \mathrm{IRun} \#$ & 9 & 10 & 11 & 12 & 13 & 14 & $14 \mathrm{a}$ & $14 \mathrm{~b}$ & $14 \mathrm{c}$ & 15 & 16 \\
\hline 2.93 & 0 & 0 & 0 & 0 & 0 & 23 & 0 & 0 & 0 & 0 & 2 \\
8.79 & 57 & 47 & 48 & 53 & 103 & 71 & 165 & 17 & 306 & 28 & 49 \\
14.65 & 37 & 18 & 88 & 33 & 91 & 40 & 216 & 23 & 179 & 29 & 34 \\
20.51 & 14 & 10 & 49 & 20 & 57 & 24 & 108 & 6 & 92 & 20 & 12 \\
26.37 & 5 & 6 & 42 & 12 & 42 & 17 & 57 & 8 & 44 & 18 & 6 \\
32.23 & 8 & 7 & 33 & 15 & 22 & 8 & 41 & 3 & 21 & 17 & 8 \\
38.09 & 9 & 3 & 22 & 13 & 29 & 5 & 27 & 5 & 18 & 8 & 4 \\
43.95 & 13 & 3 & 21 & 11 & 19 & 6 & 27 & 5 & 9 & 6 & 4 \\
49.8 & 12 & 3 & 14 & 8 & 19 & 6 & 22 & 1 & 15 & 0 & 1 \\
55.66 & 4 & 0 & 12 & 7 & 11 & 4 & 6 & 4 & 12 & 5 & 1 \\
61.52 & 1 & 2 & 2 & 3 & 10 & 6 & 6 & 0 & 10 & 2 & 1 \\
67.38 & 3 & 0 & 8 & 4 & 6 & 1 & 10 & 2 & 2 & 0 & 0 \\
73.24 & 1 & 2 & 6 & 1 & 5 & 3 & 1 & 0 & 3 & 0 & 1 \\
79.1 & 4 & 0 & 3 & 0 & 2 & 1 & 4 & 0 & 4 & 1 & 0 \\
84.96 & 1 & 1 & 0 & 1 & 2 & 2 & 1 & 2 & 4 & 1 & 1 \\
90.82 & 5 & 1 & 2 & 0 & 3 & 2 & 0 & 1 & 4 & 1 & 0 \\
96.68 & 0 & 0 & 3 & 0 & 1 & 2 & 2 & 0 & 1 & 0 & 1 \\
102.54 & 4 & 0 & 0 & 1 & 1 & 3 & 2 & 0 & 4 & 0 & 0 \\
108.4 & 1 & 1 & 0 & 3 & 3 & 3 & 0 & 0 & 4 & 1 & 0 \\
114.26 & 1 & 0 & 2 & 2 & 0 & 2 & 1 & 0 & 0 & 0 & 0 \\
120.12 & 1 & 2 & 0 & 0 & 2 & 0 & 0 & 0 & 0 & 0 & 0 \\
125.98 & 0 & 1 & 0 & 0 & 0 & 1 & 0 & 0 & 0 & 0 & 0 \\
131.84 & 1 & 0 & 0 & 0 & 0 & 0 & 0 & 1 & 1 & 0 & 0 \\
137.7 & 0 & 1 & 0 & 1 & 0 & 1 & 0 & 0 & 3 & 0 & 0 \\
143.55 & 0 & 1 & 0 & 0 & 0 & 1 & 0 & 0 & 0 & 0 & 0 \\
149.41 & 0 & 0 & 0 & 1 & 0 & 0 & 0 & 0 & 3 & 0 & 0 \\
$>150$ & 2 & 2 & 1 & 5 & 2 & 4 & 0 & 2 & 5 & 3 & 3 \\
& & & & & & & & & & &
\end{tabular}

$\begin{array}{llllllllllll}\text { Total }(\mathrm{N}) & 184 & 111 & 356 & 194 & 430 & 236 & 696 & 80 & 744 & 140 & 128\end{array}$ 
Table II-3: Measured sulfur content of individual particles for selected runs (sulfur content values given in percent).

\begin{tabular}{|c|c|c|c|c|c|c|c|c|}
\hline Run: 17a & $17 \mathrm{~b}$ & 19 & 24 & 23 & 28 & 29 & 38 & 39 \\
\hline 0.0 & 0.0 & 0.0 & 0.0 & 0.8 & 0.0 & 0.0 & 0.0 & 0.0 \\
\hline 0.0 & 0.0 & 0.0 & 0.0 & 1.4 & 0.0 & 0.0 & 0.0 & 0.0 \\
\hline 0.2 & 3.5 & 0.0 & 0.0 & 1.5 & 0.0 & 0.0 & 0.3 & 0.0 \\
\hline 1.3 & 3.5 & 0.0 & 2.2 & 3.0 & 0.9 & 0.6 & 1.0 & 0.1 \\
\hline 1.7 & 4.9 & 6.2 & 4.7 & 6.4 & 2.5 & 3.7 & 1.0 & 0.9 \\
\hline 2.2 & 5.0 & 10.6 & 5.7 & 6.8 & 10.0 & 4.9 & 3.2 & 2.2 \\
\hline 2.5 & 5.5 & 12.1 & 8.5 & 9.4 & 12.5 & 5.6 & 4.2 & 3.4 \\
\hline 6.0 & 7.0 & 13.9 & 15.3 & 11.2 & 13.5 & 6.0 & 4.2 & 4.4 \\
\hline 7.3 & 8.9 & 14.5 & 16.3 & 12.2 & 14.6 & 9.0 & 8.3 & 6.0 \\
\hline 7.7 & 11.1 & 16.5 & 16.7 & 13.8 & 14.7 & 10.9 & 9.1 & 7.7 \\
\hline 11.9 & 11.4 & 17.5 & 17.2 & 14.0 & 15.2 & 12.0 & 9.6 & 8.5 \\
\hline 15.2 & 12.6 & 23.2 & 18.9 & 17.3 & 15.4 & 13.3 & 11.2 & 8.7 \\
\hline 16.5 & 12.7 & 25.3 & 19.4 & 20.6 & 17.5 & 13.5 & 11.6 & 10.0 \\
\hline 19.4 & 14.4 & 32.3 & 19.6 & 23.1 & 17.7 & 13.6 & 11.7 & 10.6 \\
\hline 19.7 & 15.3 & 32.6 & 19.7 & 23.3 & 17.9 & 13.9 & 12.6 & 10.8 \\
\hline 20.5 & 15.8 & 33.9 & 21.9 & 23.9 & 18.4 & 16.1 & 13.0 & 11.5 \\
\hline 20.6 & 19.4 & 34.6 & 23.6 & 25.7 & 18.7 & 16.6 & 13.4 & 13.4 \\
\hline 20.6 & 20.1 & 34.8 & 23.7 & 27.6 & 19.3 & 17.5 & 16.5 & 14.0 \\
\hline 21.5 & 21.0 & 36.5 & 25.1 & 29.7 & 19.4 & 19.2 & 17.1 & 14.1 \\
\hline 22.0 & 22.7 & 36.7 & 25.7 & 30.1 & 20.6 & 19.8 & 17.2 & 17.1 \\
\hline 24.0 & 24.2 & 37.8 & 26.0 & 32.0 & 21.1 & 19.9 & 19.2 & 17.4 \\
\hline 25.0 & 26.7 & 38.3 & 26.2 & 34.3 & 21.7 & 21.5 & 19.4 & 17.7 \\
\hline 26.3 & 28.8 & 38.7 & 28.9 & 35.1 & 22.1 & 23.3 & 20.1 & 18.0 \\
\hline 28.6 & 29.4 & 39.0 & 30.9 & 37.6 & 22.6 & 23.5 & 20.9 & 19.0 \\
\hline 29.5 & 29.5 & 40.2 & 32.1 & 38.8 & 22.9 & 23.9 & 21.9 & 19.1 \\
\hline 29.8 & 30.5 & 40.8 & 32.2 & 38.8 & 23.0 & 26.6 & 22.2 & 19.7 \\
\hline 30.8 & 32.0 & 41.2 & 34.7 & 40.0 & 23.7 & 29.1 & 24.7 & 22.3 \\
\hline 32.4 & 32.9 & 41.4 & 35.2 & 42.8 & 26.0 & 29.5 & 26.6 & 24.2 \\
\hline 33.3 & 33.3 & 42.7 & 35.5 & 48.3 & 26.1 & 32.3 & 26.8 & 24.3 \\
\hline 35.3 & 34.9 & 42.7 & 37.5 & 49.0 & 26.8 & 32.4 & 27.1 & 24.8 \\
\hline 36.0 & 35.7 & 43.7 & 37.8 & 49.9 & 27.4 & 33.1 & 31.3 & 26.9 \\
\hline 39.0 & 36.1 & 43.8 & 37.9 & 50.6 & 27.5 & 33.5 & 31.6 & 27.0 \\
\hline 40.1 & 36.8 & 45.2 & 39.4 & 52.5 & 27.9 & 33.9 & 31.8 & 28.0 \\
\hline 41.4 & 37.0 & 46.9 & 40.3 & 55.8 & 28.5 & 33.9 & 34.5 & 28.3 \\
\hline 42.5 & 38.6 & 47.7 & 40.6 & 58.6 & 29.2 & 34.2 & 35.6 & 31.0 \\
\hline 43.4 & 38.9 & 48.2 & 41.3 & 65.4 & 30.6 & 35.1 & 35.9 & 34.0 \\
\hline 44.2 & 39.3 & 48.7 & 44.8 & 65.5 & 32.9 & 35.7 & 40.0 & 34.6 \\
\hline 46.4 & 47.8 & 53.3 & 45.2 & 66.6 & 35.0 & 38.0 & 42.6 & 36.5 \\
\hline 46.9 & 54.6 & 56.5 & 46.4 & 68.2 & 40.0 & 38.1 & 53.2 & 41.5 \\
\hline 49.6 & 58.3 & 58.6 & 57.4 & 68.5 & 50.3 & 40.7 & 55.1 & 47.1 \\
\hline
\end{tabular}


Table II-4: Predicted sulfur content of individual particles for selected continuous runs (sulfur content values given in percent).

\begin{tabular}{|c|c|c|c|c|c|c|}
\hline Run: 17 & 19 & 24 & 23 & 28 & 29 & 38 \\
\hline 0.1 & 0.5 & 1.1 & 3.4 & 0.7 & 0.2 & 0.0 \\
\hline 3.2 & 3.7 & 2.8 & 4.8 & 5.0 & 2.8 & 0.5 \\
\hline 3.6 & 4.3 & 4.7 & .4 .8 & 5.0 & 2.8 & 1.9 \\
\hline 3.7 & 7.2 & 4.7 & 5.1 & 5.5 & 3.0 & 3.5 \\
\hline 4.2 & 7.7 & 4.9 & 5.7 & 7.1 & 4.6 & 3.6 \\
\hline 6.8 & 9.1 & 5.4 & 7.0 & 9.6 & 5.4 & 4.4 \\
\hline 7.0 & 11.3 & 6.8 & 9.0 & 10.7 & 6.0 & 5.5 \\
\hline 8.3 & 12.5 & 8.6 & 9.3 & 13.2 & 7.6 & 6.7 \\
\hline 10.1 & 13.7 & 9.1 & 10.2 & 14.0 & 8.1 & 6.9 \\
\hline 10.5 & 15.1 & 9.6 & 11.3 & 15.0 & 8.7 & 7.4 \\
\hline 11.1 & 16.3 & 10.8 & 13.1 & 16.7 & 10.1 & 8.4 \\
\hline 12.5 & 17.5 & 12.8 & 13.5 & 17.6 & 10.6 & 9.7 \\
\hline 13.4 & 18.8 & 13.2 & 14.4 & 18.9 & 11.4 & 10.2 \\
\hline 14.1 & 20.0 & 13.9 & 16.0 & 20.0 & 12.3 & 11.2 \\
\hline 15.2 & 21.0 & 15.1 & 17.1 & 20.9 & 13.1 & 12.0 \\
\hline 16.3 & 22.4 & 16.6 & 18.0 & 21.9 & 14.1 & 12.6 \\
\hline 17.4 & 24.0 & 17.5 & 19.2 & 23.5 & 15.0 & 13.2 \\
\hline 18.6 & 25.1 & 18.4 & 20.5 & 24.9 & 15.7 & 13.8 \\
\hline 19.6 & 26.4 & 19.6 & 21.8 & 26.5 & 16.4 & 14.5 \\
\hline 20.4 & 28.1 & 20.6 & 22.7 & 27.7 & 17.2 & 15.3 \\
\hline 21.4 & 29.2 & 21.7 & 23.8 & 28.7 & 18.0 & 16.4 \\
\hline 22.2 & 30.2 & 22.7 & 25.0 & 30.1 & 19.2 & 17.2 \\
\hline 23.2 & 31.6 & 23.6 & 26.2 & 31.5 & 20.2 & 17.9 \\
\hline 24.3 & 32.5 & 24.9 & 27.3 & 32.4 & 21.1 & 18.5 \\
\hline 25.0 & 33.8 & 26.2 & 28.6 & 33.5 & 21.9 & 19.5 \\
\hline 26.1 & 35.1 & 27.5 & 29.9 & 34.6 & 22.8 & 20.1 \\
\hline 27.3 & 36.4 & 28.8 & 31.4 & 35.7 & 23.9 & 21.3 \\
\hline 28.3 & 37.6 & 30.2 & 32.7 & 36.8 & 24.7 & 22.4 \\
\hline 29.3 & 38.5 & 31.0 & 34.2 & 37.7 & 25.2 & 23.3 \\
\hline 30.4 & 39.7 & 32.4 & 35.3 & 39.4 & 26.7 & 24.0 \\
\hline 31.0 & 41.1 & 33.3 & 36.4 & 40.6 & 28.5 & 24.9 \\
\hline 32.3 & 42.2 & 34.3 & 37.4 & 41.6 & 29.7 & 26.6 \\
\hline 34.3 & 43.3 & 35.7 & 38.9 & 43.0 & 30.8 & 27.8 \\
\hline 35.4 & 44.2 & 37.0 & 39.9 & 44.6 & 31.9 & 28.6 \\
\hline 36.5 & 45.7 & 38.0 & 41.7 & 45.7 & 32.9 & 29.7 \\
\hline 37.5 & 46.8 & 39.6 & 43.0 & 47.0 & 34.5 & 31.1 \\
\hline 38.7 & 48.2 & 40.3 & 44.3 & 48.0 & 35.3 & 32.1 \\
\hline 39.9 & 50.1 & 41.8 & 45.6 & 49.7 & 36.6 & 33.0 \\
\hline 41.4 & 51.1 & 43.2 & 47.0 & 51.2 & 37.9 & 33.6 \\
\hline 42.5 & 52.7 & 44.1 & 48.0 & 52.4 & 39.3 & 34.9 \\
\hline 43.3 & 54.4 & 45.4 & 49.0 & 54.2 & 40.8 & 36.8 \\
\hline
\end{tabular}


Table II-4 continued.

$\begin{array}{lllllll}45.2 & 55.2 & 46.8 & 50.7 & 55.6 & 43.2 & 38.3 \\ 46.6 & 56.9 & 49.2 & 53.0 & 57.3 & 44.9 & 40.2 \\ 49.6 & 59.5 & 51.0 & 54.7 & 59.0 & 46.9 & 41.5 \\ 51.7 & 61.0 & 52.9 & 57.4 & 60.7 & 48.3 & 43.0 \\ 54.0 & 63.2 & 55.1 & 58.8 & 63.5 & 50.7 & 45.0 \\ 56.0 & 65.2 & 57.9 & 60.9 & 65.8 & 53.3 & 47.3 \\ 58.7 & 69.3 & 61.0 & 64.3 & 68.2 & 56.6 & 52.0 \\ 61.8 & 72.6 & 65.8 & 69.3 & 71.3 & 61.7 & 55.9 \\ 75.5 & 83.9 & 85.4 & 87.9 & 83.5 & 72.3 & 66.0\end{array}$

Table II-5: Measured sulfur content of individual particles for batch runs shown in Figures 4.3 and 5.25 (sulfur content values given in percent).

\begin{tabular}{|c|c|c|c|c|c|c|c|}
\hline \multirow{2}{*}{\multicolumn{4}{|c|}{ Data given in Figure 4.3}} & \multicolumn{4}{|c|}{ Data given in Figure 5.25} \\
\hline & & & & \multicolumn{2}{|c|}{$t=210 \mathrm{~s}$} & \multicolumn{2}{|c|}{$t=480 \mathrm{~s}$} \\
\hline 4.3 & 20.6 & 24.7 & 28.2 & 0.7 & 10.3 & 3.2 & 23.1 \\
\hline 9.4 & 20.7 & 25.0 & 28.5 & 1.2 & 11.9 & 3.6 & 24.0 \\
\hline 11.4 & 20.7 & 25.1 & 28.6 & 1.3 & 11.9 & 4.1 & 24.9 \\
\hline 11.6 & 20.9 & 25.1 & 28.8 & 1.9 & 12.3 & 5.0 & 25.9 \\
\hline 13.1 & 21.0 & 25.2 & 28.8 & 2.0 & 12.6 & 5.7 & 26.1 \\
\hline 13.8 & 21.6 & 25.3 & 28.9 & 2.2 & 13.2 & 6.0 & 26.7 \\
\hline 15.1 & 22.0 & 25.7 & 29.4 & 2.6 & 13.7 & 7.1 & 28.1 \\
\hline 16.6 & 22.0 & 25.7 & 29.4 & 3.1 & 14.5 & 8.7 & 28.5 \\
\hline 17.0 & 22.2 & 25.8 & 29.5 & 3.4 & 14.6 & 8.7 & 29.0 \\
\hline 18.3 & 22.3 & 25.9 & 30.3 & 3.7 & 15.9 & 8.9 & 29.2 \\
\hline 18.7 & 22.5 & 26.0 & 30.5 & 3.9 & 16.8 & 10.2 & 29.8 \\
\hline 18.7 & 22.6 & 26.6 & 30.6 & 4.0 & 16.9 & 13.3 & 30.1 \\
\hline 19.1 & 22.8 & 26.9 & 30.7 & 5.4 & 17.4 & 13.4 & 31.0 \\
\hline 19.3 & 22.8 & 27.2 & 31.3 & 5.4 & 17.7 & 15.0 & 31.4 \\
\hline 19.3 & 22.8 & 27.3 & 31.5 & 5.4 & 17.8 & 16.1 & 31.7 \\
\hline 19.5 & 23.0 & 27.3 & 31.5 & 6.9 & 17.8 & 16.6 & 32.0 \\
\hline 19.5 & 23.3 & 27.5 & 32.0 & 7.5 & 17.9 & 17.4 & 33.7 \\
\hline 19.5 & 23.3 & 27.6 & 32.2 & 8.1 & 18.2 & 18.5 & 33.8 \\
\hline 19.8 & 23.4 & 27.6 & 32.3 & 8.4 & 19.3 & 18.6 & 34.9 \\
\hline 20.0 & 23.4 & 27.7 & 33.4 & 8.5 & 19.7 & 21.2 & 35.6 \\
\hline 20.0 & 24.7 & 28.0 & 34.3 & 8.5 & 21.2 & 22.0 & 37.6 \\
\hline 20.0 & 24.7 & 28.0 & 34.4 & 9.4 & 22.5 & 22.1 & 38.5 \\
\hline 20.0 & 24.7 & 28.1 & 34.8 & 9.4 & 26.9 & 22.2 & 39.5 \\
\hline 20.2 & 24.7 & 28.1 & 37.6 & 10.0 & 27.1 & 22.5 & 39.8 \\
\hline 20.5 & 24.7 & 28.2 & 37.6 & 10.2 & 33.8 & 22.9 & 40.2 \\
\hline
\end{tabular}




\section{Appendix III: \\ Computer Program Listings}

The source code listings (written in FORTRAN) and a description of the main computer program used to determine the coating distribution and product quality are given in this section. The numerical procedure, solution methods and definitions of selected variables in the COMMON statements used in the program are described first. The computer program represents Model IV and includes hydrodynamics, spray distribution and Monte Carlo models.

The procedure for solving Model IV including the solution methods for solving the mass and momentum equations in the spout and the fluid flow in the annulus are given below:

\section{Numerical Procedure}

(i) Read in the experimental data, convert equipment units to metric units using calibration equations, and determine properties of air using equations of state.

(ii) Calculate bed parameters such as $U_{m f} U_{m s}, v_{t}$ and $D_{s}$;

(iii) Determine the axial pressure distribution $\left(P_{a}\{z\}\right)$ using Equations (2.11) and (4.23), and calculate the values of SPLINE parameters (cubic interpolation parameters);

(iv) Solve for the steam function $(\psi)$ using Equation (I-23), velocities $\left(u_{a}\{z, r\}\right)$ using Equation (I-22) and pressures $\left(P_{a}\{z, r\}\right)$ in the annulus using Equation (I-28); determine the fluid flow rate across the spout-annulus interface and calculate the values of SPLINE parameters for the exchange flux $U_{r}$;

(v) Solve Equations (4.27), (4.29), (4.30), (4.36) and (4.37) to determine $\varepsilon_{s}, v_{s} u_{s}$ and $C_{s}$ and determine the corresponding values of SPLINE parameters;

(vi) Calculate $m_{s l}$ at various positions on the surface of the bed $\left(D_{r}\right)$ and the values of SPLINE parameters; 
(vii) Determine the particle sulfur content distribution with the Monte Carlo methods outlined in Section 4.2.3.3.

In all steps, the calculated information is stored as SPLINE parameters which provide this information whenever it is required through a simple calculation.

Solution Methods for Steps (iv) and (v)

In step (iv), the vector Ergun (1952) equation was written in terms of the stream function (i.e., Equation (I-23)) which was then expressed in second order finite differences forms and solved using an successive under-relaxation factor of 0.7 . The grid size was set in such a way that nodes were located on the conical wall. Twenty-one grids (between $D$ and $D_{s}$ ) were used in the radial direction, and the number of grids in the axial direction was calculated from the number of grids in the radial direction, cone angle, $H$ and $D$. In most cases, the number of grids in the axial direction exceeded that in the radial direction. The convergence criterion of of $10^{-9}$ for the maximum change in the stream function value from iteration to iteration was used. (Decreasing this value to $10^{-10}$ did not significantly improve the results, but the number of iterations required to meet the criterion nearly doubled). For most runs, convergence was reached in under 5000 iterations using an under-relaxation factor of 0.7 . No attempt was made to find the optimum relaxation factor; however, the rate of convergence appeared to be slower with smaller values, and larger values resulted in no convergence.

The same method was applied for solving the pressure profile in the annulus (i.e., Equation (I28)), except that linear or first order approximations were used to predict the normal pressure derivative (Equation (I-29)) at the conical wall.

In step (v), the set of non-linear, coupled ordinary differential equations were solved using LSODE (in UBC ODEPACK, Moore, 1989) which utilizes the Gear method (Gerald and Wheatley, 1984). The solution could be obtained in most cases provided the equations were rewritten (and solved) in terms of $u_{s}, u_{s} \varepsilon_{s}$ and $v_{s}^{2}\left(1-\varepsilon_{s}\right)$. Absolute and relative tolerance values of $10^{-12}$ and $10^{-5}$, respectively, were used. 
Variables in the COMMON statements

'H.COM1' contains operating variables, bed constants and properties of bed particles:

AAO Cross-sectional area of the annulus in the cylindrical section

AAB Cross-sectional area of the cylindrical portion of the bed above the shutter and below the cone

AR Archimedes number

AS Cross-sectional area of the spout

CK1,CK2 Coefficients of the Ergun (1952) equation

DB Diameter of the cylindrical portion of the bed above the shutter and below the cone

DCOL $\quad D_{c}$

DI $\quad d_{i}$

DIST $\quad d_{i}^{\prime}$

DNI Diameter of the atomizing air orifice for the air cap of the nozzle

DP $\quad d_{p}$

DPF $\quad \Delta P_{f} ;$ Epstein and Grace (1984)

DPU. Diameter of urea particles

DS $\quad D_{s}$

ETAC $\quad \eta$

GRAV $g$

$\mathrm{H} \quad H$

HB Length of the cylindrical portion of the bed above the shutter and below the cone

HCOAT $h_{f d s}$

HHM $\quad H / H_{m}$

HM $\quad H_{m} ;$ McNab and Bridgwater (1977)

LFEED Feed type code - forced or arbitrary feed of urea

NDS Dimension of the arrays used for all SPLINE routines

NPRINT Print code

$\mathrm{PH} \quad P_{H}$

$\mathrm{PI}, \mathrm{PI} 4 \pi, \pi / 4$

PSI Sphericity of bed particles

QA $\quad Q_{a}$

QS $Q_{s}$

QT $Q_{t}$

$\mathrm{RHO} \rho$ 
RHOAT Density of atomizing air

RHOP $\quad \rho_{p}$

RHOSF Density of liquid sulfur @ $150^{\circ} \mathrm{C}$

RHOSM Density of monoclinic sulfur

RHOSR Density of rhombic sulfur

RHOU Density of solid urea

$\mathrm{T} \quad T_{b}$

TA Temperature of atomizing air

TV Terminal velocity based on the average bed particle size; Grace (1986)

TAN30 $\tan \left(30^{\circ}\right)$

THETA Included cone angle

UAHM $U_{a H m}=0.9 U_{m r}$, Epstein et al. (1978); Superficial gas velocity at maximum spoutable bed depth

UCOL Superficial air velocity based on the column diameter

UMF $\quad U_{m f} ;$ Grace (1982)

UMSMG $U_{m s}$ based on the Mathur-Gishler equation

UNIVC Universal gas constant

VISC $\mu$

VOID $\varepsilon_{m f}$

WMA Molecular weight of air

WS $\quad W_{s}$

WU $\quad W_{u}$

WTOT $\quad W_{i}$; Production rate of sulfur coated urea

XSM $\quad \bar{X}_{s m}$

XS $\quad \bar{X}_{s}$

XUD $\quad U_{D}$; Seven day dissolution of urea in the product

'H.COM4' includes the following arrays that are used in SPLINE routines:

$\begin{array}{ll}\text { CS } & C_{s} \\ \text { CZ } & z \\ \text { ES } & \varepsilon_{s} \\ \text { NSP } & \text { Dimension of } R \text { and } Z \text { arrays } \\ \text { PAN } & P_{a} \\ \text { QAN } & Q_{a}\end{array}$




$\begin{array}{ll}\text { R } & \text { Radial distance from the centre of the bed } \\ \text { SMT } & \text { Amount of sulfur on a bed particle calculated by the Monte Carlo method } \\ \text { VS } & v_{s} \\ \text { Z } & \text { Vertical distance from the base of the bed } \\ \text { ZMT } & \text { Vertical distance corresponding to SMT }\end{array}$


PROGRAM SPCOAT

IMPLICIT REAL*8(A-H,O-Z)

INCLUDE 'H.COM1'

REAL AAR(6),BB(20,6),AAZ(20),PLX(21, 101)

COMMON /GRIM/ PLX,AAZ,AAR,BB,NA,NS,IRO,IZO,NR

CALL INITIA

WRITE $\left.(8,)^{\prime}\right)^{\prime}$ VOID $=$ ', VOID

$\mathrm{KK}=0$

NPRINT $=0$

$10 K K=K K+1$

IF(KK.EQ.20) THEN

CALL PRINTO

STOP

ENDIF

NPRINT $=$ NPRINT + 1

CALLL DATINS

CALL STREAM

C IF(KK.GE.2) CALL PLOTA2

CALL PLOTS1

CALL PLOTMT

GOTO 10

END

\section{SUBROUTINE DATINS}

IMPLICIT REAL*8(A-H,O-Z)

INCLUDE 'H.COM1'

READ(5, ",END=5)NRUN, IDI,R1, RA, RS, FU,TB,IDCOL,IH,XSM,XUD,IC,WTOT WRITE(8,")NRUN,IDI,R1,RA,RS,FU,TB,IDCOL,IH,XSM,XUD,IC,WTOT GOTO 20

5 CONTINUE

C CALL PLOTAL

WRITE $(8, *)^{\prime}$ * That"s All Folks! **1

CALL PRINTO

STOP

20 CONTINUE

WS $=$ CAL( $4, R S, R S)$

$\mathrm{WU}=\mathrm{CAL}(5, \mathrm{FU}, \mathrm{FU})$

CALL DIPRTY(IH,IDCOL,IDI,IC)

WRITE $\left(8,{ }^{*}\right)^{\prime} \mathrm{RUNH}, \mathrm{H}, \mathrm{D}, \mathrm{DI}, \mathrm{WU}, W S, W T O T, X S, X S M, D P={ }^{\prime}, \mathrm{NRUN}, \mathrm{H}, \mathrm{DCOL}$

+ DI,WU,WS,WTOT,XS,XSM,DP

IF(IDCOL.EQ.2) DCOL $=$ DCOL $* 0.81$

$T=T B+273$

$T A=180 .+273$

CALL EOS(T,RHO,VISC)

CALL EOS(TA, RHOAT,VISCAT)

$Q A=C A L(3, R A, R H O A T)$

$Q S=C A L(1, R 1, R H O)$

$Q T=Q A+Q S$

$X U D=X U D / 100$

$\mathrm{PH}=0$.

WRITE $(8, *)^{*} T, T A, R H O, V I S C=$, T, TA, RHO,VISC

$\mathrm{UCOL}=\mathrm{QT} / \mathrm{PI} / \mathrm{DCOL}$ * 2

IF (DI.GT.25. "DP) WRITE(7,*)'DI/DP > 25, UNSTABLE BED'

$\left.A R=D P^{* *} 3^{*}(R H O P-R H O)\right)^{*}$ GRAV * RHO / VISC**2

$H M=D C O L * 2 / D P *(D C O L / D I) * *(2 . / 3) * 700.1 A$.$R *$

( $\left.\left(1 .+35.9 E-6^{*} A R\right)^{* *} .5-1.\right)^{* * 2}$

RHOB $=(1 .- \text { VOID })^{*}$ RHOP

$\mathrm{GU}=\mathrm{RHO} \cup \mathrm{CO}$

WRITE $(8, *)^{\prime} 1111$, RHOB, VOID, RHOP= ' RHOB, VOID, RHOP

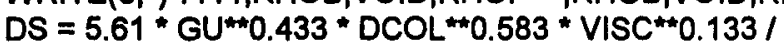
(RHOB*RHO*GRAV)**0.283

WRITE(8,")'2222, VISC,AR, RHOB,QS= ',VISC,AR,RHOB, QS

CANGLE $=20$

HCOAT $=$ DS/2./DATAN(CANGLE/180.*P1/2.) 
WRITE $(8, *)$ 'HCOAT,CANG,DS = ',HCOAT,CANGLE,DS

$A S=D S^{* * 2 *} P \mid 4$

$A A B=P 14^{*}\left(D B^{*} D B-D S^{*} D S\right)$

$A A O=P \mid 4^{*}(D C O L * * 2-D S * D S)$

$P M A X=H^{*}(R H O P-R H O)$ * (1.-VOID) * GRAV

$X 11=Q A N\left(.1^{*} .0254\right)^{* * 2} 2^{*} \mathrm{DI} \mathbf{N}^{* *} 2^{*} \mathrm{QS}$

CALL SUBUMS(DIST,UMS)

CALL SPRAY(QAMIN,QAMAX)

C $\quad D I=D I S T$

ETAC = DSP**2*RHOSF/18.NISC/DP

DPST $=$ DP* $\left(R H O O^{*} \text { GRAV*}^{*}(\mathrm{RHOP}-\mathrm{RHO}) \mathrm{NISC}^{* *} 2\right)^{* *}(1 . / 3$.

UMFST $=$ DSQRT $\left(27.2 * \star 2+.0408^{\star} A R\right)-27.2$

STO $=\left(R_{H O *} 2 N I S C / G R A V /(R H O P-R H O)\right)^{\star \star}(1 . / 3$.

UMF = UMFST/STO/DPST

WDP $=$ DLOG 10 (DPST)

VTST $=10^{* *}\left(-1.64758+2.47864^{*} W D P-1.09703^{*} W D P^{* * 2}\right.$ $+0.17129 * W D P * * 3$ )

TV $=$ VTST/STO

UAHM $=0.9 *$ UMF

$H H M=H / H M$

DPPSI=DP*PSI

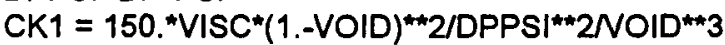

CK2 $=1.75^{*}$ RHO $^{*}(1 .-$ VOID $) / D P P S I N O D^{* * 3}$

$\mathrm{DPF}=-\mathrm{H}^{*}(\mathrm{CK} 1+\mathrm{CK} 2 * \mathrm{UMF})^{*} \mathrm{UMF}$

WRITE (7,65)NRUN,DP, RHOP,RHO,QT,QA,H,DCOL,DI,VOID,PSI

C WRITE(11,66)RUNNO(1:8),PRTYPE(1:12),QT,QA,H,DCOL,DI,RHO WRITE(7,67)WS, WU,DSP, QAMIN, QAMAX,DIST,XS,XSM,XUD,UMS WRITE(7,70)DS, UCOL, UMF, UAHM, TV, HM,DPF, PMAX,CK1,CK2 65 FORMAT('1' $/$ th Run \# ',12/' Dp = ',F6.4,' Rhop = ',F5.0,

+ 'Rho $=$ 'F4.2' ${ }^{\prime}$ Qs $=$ ' $F 7.5$,

+' $\mathrm{Qa}=$ ',F7.5' H = ',F4.2, $\mathrm{D}=$ =',F4.2,' Di = ',F6.4,

+ ' Void = ',F5.3,' Psi (Sphericity) = ',F4.2)

66 FORMAT(A9,A14,2X,F7.5,F9.5,F6.2,F6.2,F8.4,F6.2) 67 FORMAT(' Ws = 'F7.5,' Wu = 'F7.5,' DSP(Spray) $=$ ',F9.71

+ ' Qamin = ',F7.5,' Qamax = ',F7.5,' Di" = ',F6.41

+ 'Xs = ',F4.3,' Xsm = ',F4.3,' Xud =',F4.3,'Ums = ',F7.5)

70 FORMAT(' Ds = ',F6.4,' Ucol = ',F5.3,' Umf = ',F5.3,

+' UaHm = ',F5.3r TV (Term Vel.) = ',F4.2,' $\mathrm{Hm}=$ ',

+ F4.2,' Dpf (dPmf) = ',F7.1, Pmax = ',F6.1/

+ 'CK1 (Ergun) = ',F6.1,' CK2 (Ergun) = ',F6.1,II)

RETURN

END
SUBROUTINE DIPRTY(IH,IDCOL,IDI,IC)

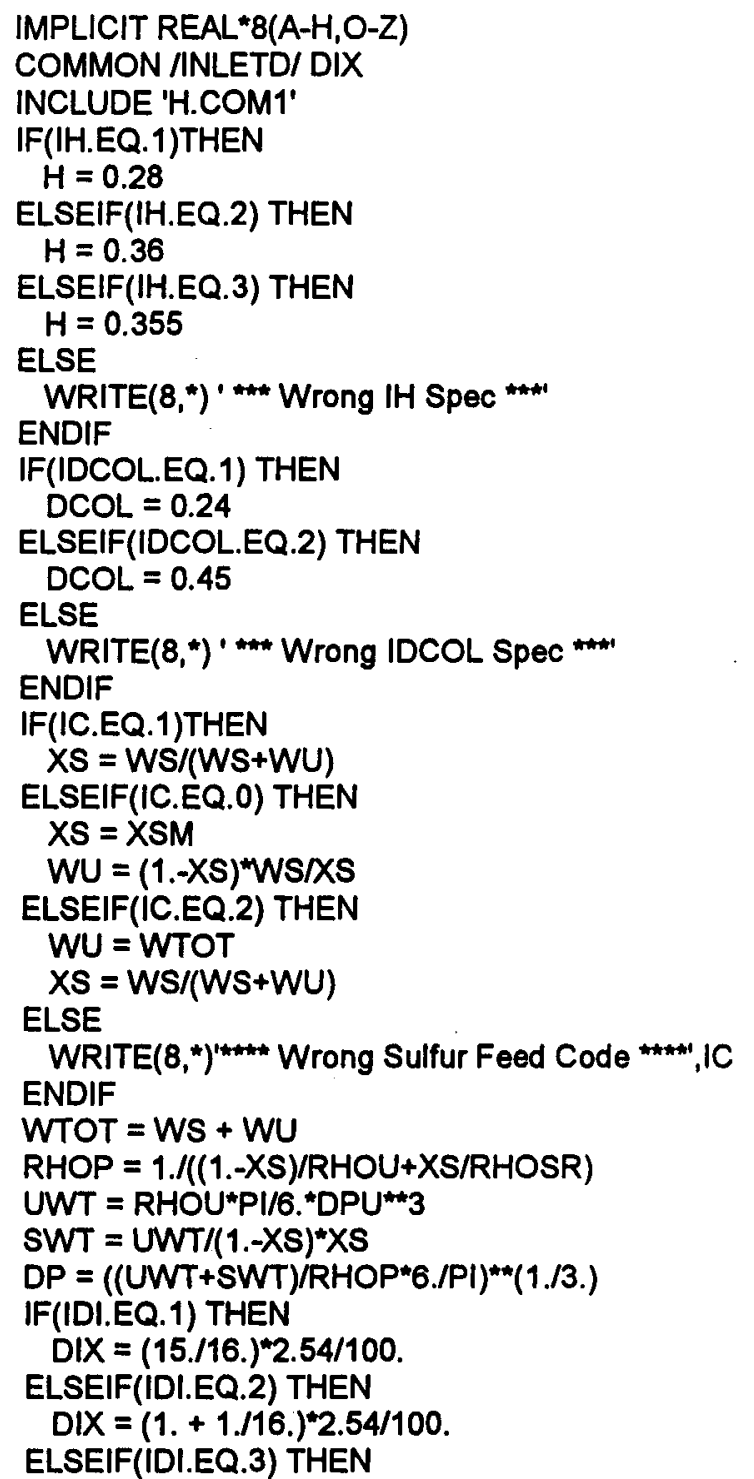


$D I X=(1 .+3 . / 16 .)^{\star} 2.54 / 100$.

ELSEIF(IDI.EQ.4) THEN

$D I X=(1 .+9 . / 32 .)^{*} 2.54 / 100$

ELSEIF(IDI.EQ.5) THEN

$D I X=(1 .+7 . / 16 .)^{\star 2} 2.54 / 100$

ELSE

WRITE $(8, *)^{* * * * * *}$ Wrong DI Spec *****1 STOP

ENDIF

$D I=C A L(8, D I X, D I X)$

END

\section{SUBROUTINE SUBUMS(DIPR,UMS)}

IMPLICIT REAL*8(A-H,O-Z)

EXTERNAL FUMS

INCLUDE 'H.COM1'

DIMENSION WORK(80)

$X=D I$

$N F=1$

SCALE $=1 . D 10$

EPSL $=1 . D-10$

IC $=1$

$\mathrm{Ml}=\mathbf{5 0 0}$

LOG $=6$

CALL DPOWEL(FM,X,NF, SCALE,EPSL,IC,MI,LOG,WORK,IE,FUMS, *32)

GOTO 33

32 CONTINUE

WRITE $\left(7,{ }^{\prime}\right)^{\prime}$ error in POWEL, IE = ',IE

33 CONTINUE

DIPR $=X$

GH = DSQRT(2. *GRAV*H)

UMS $=13.50^{*}(\mathrm{DP} / \mathrm{DCOL})^{\star \star 1} 1.165{ }^{*}(\mathrm{DIPR} / \mathrm{DCOL}){ }^{\star \star 0} 0.3715$

$+\quad *(\mathrm{DCOL} / \mathrm{H}){ }^{\star *} 0.1475^{*}((\mathrm{RHOP}-\mathrm{RHO}) / \mathrm{RHO}){ }^{* *} 0.2894^{*} \mathrm{GH}$

WRITE $\left(8,{ }^{*}\right)^{\prime}$ 'values Of $F M, X$ are ',FM, $X$

END 
DOUBLE PRECISION FUNCTION FUMS $(X, N D)$

IMPLICIT REAL $*$ (A-H,O-Z)

INCLUDE 'H.COM1'

$V A=Q A P \mid 4 / D A T^{\star *} 2$

$A I=P \mid 4^{*} D I^{* * 2}$

$\mathrm{GH}=\mathrm{DSQRT}\left(2 .{ }^{*} \mathrm{GRAV}{ }^{\star H}\right)$

$X X=X$

IF(X.LE.0.) THEN

FUMS $=-X^{\star} 10000 .+1000$

RETURN

ENDIF

UMS $=13.50 *(D P / D C O L) \star \star 1.165 *(X X / D C O L) \star \star 0.3715$

$+\quad(D C O L / H)^{\star \star} 0.1475^{\star}((\mathrm{RHOP}-\mathrm{RHO}) / \mathrm{RHO})^{\star \star} 0.2894^{\star} \mathrm{GH}$

QMS $=\mathrm{UMS}^{\star} \mathrm{PI}^{\star}{ }^{\star} \mathrm{DCOL}^{* \star 2}$

$Q T X=Q M S+Q A$

$V T X=Q T X|X X * 2 / P| 4$

QMS2 = QTX*VTX*Al-(WS*VA+RHOAT*QA*VA)*AI/RHO

IF(QMS2.LE.0.) QMS2 = -QMS2*1000.

QMS1 = DSQRT(QMS2)

FUMS $=((Q M S-Q M S 1) * 1000 .)^{* * 2}$

RETURN

END
SUBROUTINE SPRAY(QAMIN, QAMAX)

IMPLICIT REAL*8(A-H,O-Z)

INCLUDE 'HCOM1'

UAMIN $=13.7893+1.6086^{*} \mathrm{WS}^{\star} 3600$

UAMAX $=3.881397+4.592215^{\star} W S^{\star} 3600$.

QAMIN = UAMIN*DAT*2*PI4

QAMAX = UAMAX*DAT**2*PI4

DSP $\left.=0.261012^{\star}(\text { QAQAMIN })^{\star \star 1.7015 *(W S / R H O S F / Q A ~}\right)^{\star \star 1.8759}$ RETURN

END 
SUBROUTINE HSPOUT

IMPLICIT REAL ${ }^{\star 8}(A-H, O-Z)$

EXTERNAL FUNC

INCLUDE 'H.COM1'

INCLUDE 'H.COM4'

DIMENSION Y(4), RTOL(4), ATOL(4), RWORK(80),IWORK(25), IPAK(100)

DATA RTOL $4 * 1 . D-5 / A T O L / 4 * 1 . D-12 /$ TTOL,ITASK, ISTATE, IOPT/1,1,1,0/

DATA LRW,LIW,MF/80,25,22/MUA/4/

* Solve the Lefroy Davidson Equations

$$
\mathrm{UO}=\mathrm{QT} / \mathrm{PI}|\mathrm{4} / \mathrm{D}|^{* * 2}
$$

$V S(1)=0$

$U S(1)=\cup 0$

$E S(1)=1$.

CS $(1)=W S / P I 4 / D S^{* *} 2 / U_{0}$

ISTATE $=1$

WRITE $(7,102) \mathrm{HB}, 1.0, \mathrm{U}, 0 ., \mathrm{CS}(1)$

102 FORMAT(I/4X,'H',10X,'VOID.',7X,'AIR V.',6X,'PART. V.',3X, 'SULF. C'//5G12.4)

103 FORMAT(7G12.4)

$$
\begin{aligned}
& X 1=C Z(1) \\
& N=N S P
\end{aligned}
$$

$N=N S P$

C $\quad \mathrm{DCOL}=0.445$

$Y(1)=U_{0}$

$Y(2)=\cup 0$

$Y(3)=0 . D 0$

$Y(4)=C S(1)$

DO $200 \mathrm{I}=2, \mathrm{~N}$

$$
X 2=C Z(I)
$$

CALL LSODE(FUNC,MUA,Y,X1,X2,ITOL,RTOL,ATOL,ITASK,ISTATE,

1 IOPT, RWORK, LRW,IWORK, LIW,JAC, MF)

IF(ISTATE.NE.2) WRITE $(8,)^{\prime * * \star \star \star \star *}$ ISTATE $=$ ', ISTATE

$\mathrm{V} 8=0 . \mathrm{DO}$

VOIDM1 $=1 .-Y(1) / Y(2)$

IF(Y(3).GT.0.)V8 = DSQRT(Y(3)/(1.DO-VOIDM1))

IF(I/2*2.EQ.I)WRITE(7,103)X2,Y(1)/Y(2),Y(2),V8,Y(4)

IF(X2.GT.H)THEN

WRITE $(8, *)^{\prime} \mathrm{Z}>\mathrm{H}, \mathrm{Z}={ }^{\prime}, \mathrm{X} 2$

GOTO 200

\section{ENDIF}

$V S(I)=V 8$
$U S(I)=Y(2)$

$E S(1)=Y(1) /(2)$

$\operatorname{CS}(1)=Y(4)$

$\operatorname{PRINT}(1, N P R I N T, 1)=Y(4)$

200 CONTINUE

$\operatorname{PRINT}(1, N P R I N T, 1)=\mathrm{CS}(1)$

$W S L=C S(N)^{*} U S(N) * E S(N) * A S$

$W C I R C=V S(N)^{*}(1 .-E S(N))^{\star} A S^{\star} R H O P$

WPROD $=W U /(1 .-X S)$

WRITE $(7,203)$ WSL,WSLMS*100 WCIRC,WPROD

203 FORMAT( $r$ Sulfur Lost $=$ ',G12.4, $\mathrm{KG} / \mathrm{S}=$ = ', G12.4, ${ }^{\prime} \%$ '

- 'Part Circ Rate = ',G12.4,' KG/S, Prod Rate = ',G12.4)

CALL MYSPLN(CZ,CS,CQ,CR,CT,N,NDS)

CALL MYSPLN(CZ,VS, VQ,VR,VT,N,NDS)

CALL MYSPLN(CZ,ES,EQ,ER,ET,N,NDS)

CALL MYSPLN(CZ,US,UQ,UR,UT,N,NDS)

WRITE $(8, *)^{\prime}$ HSPOUT OK

RETURN

END 
SUBROUTINE FUNC(M,X,Y,Z)

* This subroutine provides RHS of the differential equations.

* Parameters are

- $\quad Z=$ array containing RHS values of differential equations.

* $X=$ independent variabLE, $Z / H$

- $Y=$ array containing dependent variables

- $M=$ number of differential equations

IMPLICIT REAL ${ }^{\star 8}(A-H, O-Z)$

REAL*8Z(M), Y(M)

INCLUDE 'H.COM1'

$E=Y(1) / Y(2)$

C WRITE( 8,1$) X, Y(1), Y(2), Y(3), E$

C 1 FORMAT(' $X, U^{\star} E, U, V 2 * E, E=', 5 G 11.4$ )

$E M=1 .-E$

$V S=0$.

IF(EM.GT.0)THEN

$V S 2=Y(3) / E M$

$\mathrm{VS}=0$.

IF(VS2.GT.0.)VS = DSQRT(Y(3)/EM)

ENDIF

IF(E.LE.0.)E $=0.000001$

DUV $=Y(2)-V S$

$U V=$ DABS(DUV) $)^{\star D U V}$

$B=F S \mid(E, 1)$

$B B=B * U V$

$D P Z=$ QAINT $(X, 10)$

DQZ $=$ QAINT $(X, 8)$

$Z(1)=-D Q Z / A S$

$Z(2)=-D P Z / Y(2) / R H O-B B / R H O / Y(1)-Z(1) / E$

$Z(3)=B B / R H O P-D P Z / R H O P{ }^{*} E M-E M^{*}(R H O P-R H O)^{*} G R A V / R H O P$

IF(X.GE.HCOAT) THEN

$Z(4)=-\left(1.5^{\star} E M^{\star} E T A(D A B S(D U V))^{\star} D A B S(D U V) / D P+Z(1)\right)^{*}$

$+Y(4) / Y(1)$

C $Z(4)=-\left(4 . / D S * D Q Z+1.5^{\star} E M^{\star} E T A(D A B S(D U V))^{\star} D A B S(D U V) / D P+Z(1)\right)^{\star}$

$C+Y(4) / Y(1)$

ELSE

$Z(4)=-Z(1)^{\star} Y(4) / Y(1)$

ENDIF

RETURN

END

Appendix III: Computer Program Listings

\section{DOUBLE PRECISION FUNCTION ETA(X)}

* Calculates impaction efficiency

IMPLICIT REAL*8(A-H,O-Z)

INCLUDE 'H.COM1'

$S S=E T A C * X$

IF(SS.LE.0.083DO)THEN

$\mathrm{ETA}=0 . \mathrm{DO}$

ELSEIF(SS.GT.0.6D0)THEN

$E T A=S S * S S /(S S+0.5 D 0){ }^{\star \star 2} 2$

ELSE

$E T A=0.036-0.2323^{\star} S S+2.422^{\star} S S^{\star} S S-2.033^{\star} S S^{\star *}$

ENDIF

END 
DOUBLE PRECISION FUNCTION FSI $(X, I)$

* FLUID-SOLID interaction term estimation.

* 1. RICHARDSON-ZAKI EQUATION

2. ERGUN'S EQUATION

- 3. MODIFIED (BY 4) R-Z EQUATION

* 4. MORGAN \& LITTMAN CORRELATION

IMPLICIT REAL*8(A-H,O-Z)

INCLUDE 'H.COM1'

IF(I.EQ.1)THEN

$F S I=0.33^{*}(1 .-X)^{*} R H O / D P / X * 1.78$

ELSEIF(I.EQ.2)THEN

$F S I=1.75^{\star}(1 .-X)^{\star}$ RHO/DP

ELSEIF(I.EQ.3)THEN

$F S I=0.109^{*}(1 .-X)^{\star} \mathrm{RHO} / D P / X * 1.64$

ELSEIF(I.EQ.4)THEN

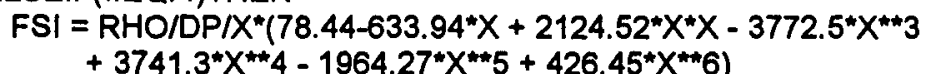

$+$

ELSE

WRITE(7,")'ERROR IN FSI CODE'

ENDIF

RETURN

END
DOUBLE PRECISION FUNCTION CAREA(X)

* This function finds the area of the annulus (cone-base).

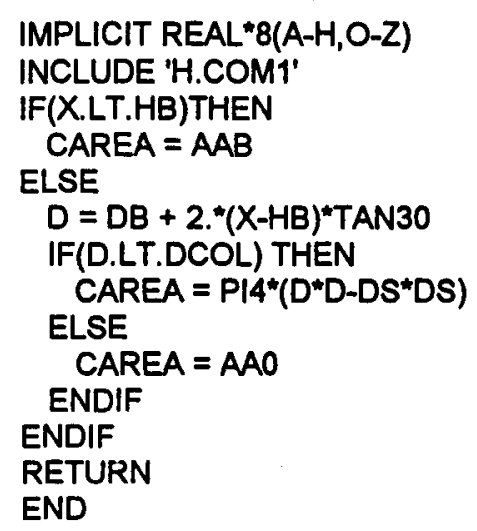




\section{FUNCTION INTT(X,Y,N,DX)}

- Determines the interval at which $X$ belongs. Used with Spline * calculations.

\section{IMPLICIT REAL*8(A-H,O-Z)}

REAL*8 Y(N)

INTT $=1$

$\mathrm{J}=\mathrm{N}-1$

$30 \mathrm{~K}=(\mathrm{INTT}+\mathrm{J}) / 2$

IF $(X . L T . Y(K)) J=K$

IF(X.GE.Y(K))INTT $=K$

IF(J.NE.INTT+1)GOTO 30

$D X=X-Y(I N T T)$

RETURN

END

\section{DOUBLE PRECISION FUNCTION QAINT(X,ICODE)}

IMPLICIT REAL*8(A-H,O-Z)

INCLUDE 'H.COM1'

INCLUDE 'H.COM4'

IF(ICODE.LT.3)THEN

$I=I N T T(X, D M T, N S P, D X)$

ELSEIF(ICODE.GE.11) THEN

$I=I N T T(X, Z, N Z, D X)$

ELSE

$I=I N T T(X, C Z, N S P, D X)$

ENDIF

IF(ICODE.EQ.1)THEN

QAINT $=Z M T(1)+Z M T Q(I)^{*} D X+Z M T R(I)^{*} D X * D X+Z M T S(I)^{*} D X * * 3$

ELSEIF(ICODE.EQ.2)THEN

QAINT = SMT $(1)+$ SMTQ $(1)^{*} D X+$ SMTR $(1)^{*} D X * D X+S M T S(I)^{*} D X * * 3$

ELSEIF(ICODE.EQ.3)THEN

C $\quad$ QAINT $=P A(1)+P Q(I)^{\star} D X+P R(1) * D X * 2+P S(1)^{\star} D X^{\star *} 3$

QAINT $=C S(1)+C Q(I) * D X+C R(I) * D X * * 2+C T(1)^{*} D X * 3$

ELSEIF(ICODE.EQ.4)THEN

QAINT $=$ US $(I)+U Q(I) * D X+U R(I)^{\star D} D X^{\star \star 2} 2+U T(I) * D X * * 3$

ELSEIF(ICODE.EQ.5)THEN

QAINT $=$ VS $(1)+V Q(I) * D X+V R(I)^{*} D X X^{*} D X+V T(I)^{*} D X * * 3$

ELSEIF(ICODE.EQ.6)THEN

QAINT $=E S(I)+E Q(I) * D X+E R(I) \star D X * \star 2+E T(I) * D X * * 3$

ELSEIF(ICODE.EQ.7)THEN

QAINT = QAN(I)+QANQ $(1)^{*} D X+Q A N R(I) * D X * 2+Q A N S(I)^{*} D X * * 3$

ELSEIF(ICODE.EQ.8)THEN

QAINT = DQAN $(1)+D Q A N Q(I) * D X+D Q A N R(I) * D X * * 2+D Q A N S(1) * D X * * 3$

ELSEIF(ICODE.EQ.9)THEN

QAINT = PAN(I)+PANQ $(1)^{*} D X+P A N R(I)^{*} D X * * 2+P A N S(1) * 0 X * 3$

ELSEIF(ICODE.EQ.10)THEN

QAINT $=$ DPAN $(I)+D P A N Q(I)^{*} D X+D P A N R(I)^{*} D X * * 2+D P A N S(I) * D X^{* * 3}$

ELSEIF(ICODE.EQ.11)THEN

QAINT $=Q Z A(I)+Q Z A Q(I)^{*} D X+Q Z A R(I)^{\star} D X *+2+Q Z A S(I)^{\star} D X^{* * 3}$

ELSEIF(ICODE.EQ.12)THEN

QAINT = RAD(I)+RADQ(I)*DX+RADR(I)*DX*2+RADS(I)*DX**3

ELSE

WRITE $(7, *)$ ' ERROR IN CODE IN QAINT, ICODE = ' ICODE

ENDIF

RETURN

END 
DOUBLE PRECISION FUNCTION PMOR(X)

IMPLICIT REAL*8(A-H,O-Z)

INCLUDE 'H.COM1'

$A C=R H O * U M F * T V /(R H O P-R H O) / G R A V / D I$

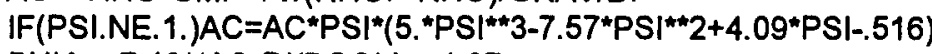

PHIA $=7.18^{*}(A C-D I / D C O L)+1.07$

IF(AC.GT.0.07)WRITE(8, $)^{\prime}$ ' P-MOR NOT VALID, AC>.07 = ',AC $X X 1=1 . /(X / D C O L+1$.

$\mathrm{XQT}=.1$

BB1 $=2{ }^{*}(X X 1-2 . * X Q T)-2 . *(1 . X Q T)+4 . *(1 .-X Q T)^{* \star 2 / P H I A}$

$C C 1=(X X 1-2 .)^{*}\left(X X 1-2 .{ }^{*} X Q T\right)-4 .^{*}(1 .-X Q T)^{* *} 2^{*} X X 1 / P H I A$

$Y 1=-B B 1 / 2 .+D S Q R T\left(B B 11^{* *} 2-4 . *^{*} C C 1\right) / 2$.

C $Y 2=-B B 1 / 2-D S Q R T\left(B B 1 * * 2-4 .{ }^{*} \mathrm{CC} 1\right) / 2$

$\mathrm{DPFO}=-\mathrm{DPF} * \mathrm{X} / \mathrm{H}$

PMOR $=(1 .-Y 1)^{*} D P F O$

RETURN

END

\section{SUBROUTINE PLOTA2}

IMPLICIT REAL*8(A-H,O-Z)

DIMENSION IPAK $(800)$

REAL AX(30),P(30),PAV(30)

INCLUDE 'H.COM1'

CALL DSPDEV('PLOT')

CALL NOBRDR

CALL AREA2D $(6.0,6.0)$

CALL PHYSOR(1.,2.)

CALL THKCRV(0.03)

CALL XREVTK

CALL YREVTK

CALL COMPLX

CALL YAXANG(0.)

CALL XNAME('Height, m\$', 100)

CALL YNAME('Pressure in Annulus, KPa\$',100)

CALL YTICKS(3)

CALL XTICKS(2)

C CALL HEADIN('Pressure Profile in Annulus $\$ 1,-100,-3,1$ )

CALL GRAF $(0,1, .4,0,3,1.3)$

CALL SCLPIC(2.)

CALL FRAME

PMORH $=$ PMOR $(H) / 1000$.

$A X(1)=0$.

$P(1)=P M O R H-P M O R(0 . D 0) / 1000$

$D O 30 \mathrm{I}=2,21$

$Z Z Z=H^{*}(1-1) / 20$

$A X(1)=Z Z Z$

$P(I)=P M O R H-P M O R(Z Z Z) / 1000$.

30 CONTINUE

CALL CURVE(AX,P,21,0)

CALL ENDGR(0)

CALL ENDPL(0)

END 
SUBROUTINE PLOTS 1

IMPLICIT REAL *8(A-H,O-Z)

DIMENSION IPAK $(500)$

REAL AX(20), V(20), VAJ(20),AX2(2)

INCLUDE 'H.COM1'

CALL DSPDEV('PLOT')

CALL NOBRDR

CALL AREA2D $(6.0,6.0)$

CALL PHYSOR(1.,2.)

CALL THKCRV(0.03)

CALL XREVTK

CALL YREVTK

CALL COMPLX

CALL YAXANG(0.)

CALL XNAME('Height, $m \$ 1,100$

CALL YNAME('Air Velocity in Spout, $\mathrm{m} / \mathrm{s} \$$ ', 100)

CALL YTICKS(3)

CALL XTICKS(2)

$\operatorname{CALL~GRAF}(0, .1,36,0.10 ., 50$.

CALL FRAME

CALL SCLPIC(2.)

CALL HSPOUT

$D X=(H-H B) / 20$.

START $=H B$

DO $201=1,20$

$X X X=S T A R T+D X *$

$A X(I)=X X X$

$V(I)=$ QAINT $(X X X, 4)$

20 CONTINUE

CALL CURVE(AX,V,20,0)

CALL ENDGR(0)

CALL ENDPL(0)

CALL DSPDEV('PLOT')

CALL NOBRDR

CALL AREA2D $(6.0,6.0)$

CALL PHYSOR $(1,2$.

CALL THKCRV(0.03)

CALL XREVTK

CALL YREVTK

CALL COMPLX

CALL YAXANG(0.)

Appendix III: Computer Program Listings
CALL XNAME('Height, m\$',100)

CALL YNAME('Concentration in Spout, $\mathrm{kg} / \mathrm{m} 3 \$ 1,100$ )

CALL YTICKS(3)

CALL XTICKS(2)

CALL GRAF(0,.1,.36,0,05,.15)

CALL FRAME

CALL SCLPIC(2.)

DO 23 I $=1,20$

$X X X=S T A R T+D X * 1$

$A X(1)=X X X$

$V(1)=$ QAINT $(X X X, 3)$

23 CONTINUE

CALL CURVE $(A X, V, 20,0)$

CALL ENDGR(O)

CALL ENDPL(0)

END 


\section{SUBROUTINE STREAM}

* This pgm determines the stream functions in the annulus using

* the boundary condition

- str=0@ wall

- $d(s t r) / d z=0 @$ annulus surface

- $P=$ function given by Morgan @ spout-annulus interface

\section{IMPLICIT REAL*8(A-H,O-Z)}

INCLUDE 'H.COM1'

INCLUDE 'H.COM4'

DIMENSION STR(101, 101),VEL(5,101,101),DPMOR(101)

DIMENSION RW(101),PMORG(101),PERG(101,101)

REAL X1,X2,Y1,Y2,RSIZ,ZSIZ,XSTEP, YSTEP

REAL AAR(6),BB(20,6),AAZ(20),PLX(21,101)

COMMON /GRIM/ PLX,AAZ,AAR, BB,NA,NS,IRO, IZO,NR

COMMON WORK $(50000)$

COMMON /FSTRSB/ F1,F2,RX,DELR,DELRZ2, ILIN

DATA NRM,EPS,MAXIT/51,1.D-9,7000/

ILIN $=1$

$N R M=20$

ALPHA $=0.7$

ALPHAM $=1$. $-A L P H A$

$A L P 2=A L P H A$

$A L P 2 M=1 .-A L P 2$

$\mathrm{F} 1=\mathrm{CK} 1$

$\mathrm{F} 2=\mathrm{CK} 2$

$\mathrm{RS}=\mathrm{DS} / 2$.

$\mathrm{RB}=\mathrm{DB} / 2$.

$\mathrm{RO}=\mathrm{DCOL} / 2$

IF(DCOL.GT.0.24) RO $=$ RO/0.81

$N R=N R M+1$

$N R P=N R+1$

NRMM $=N R-2$

DELR $=($ RO-RS $) / N R M$

DELR2 = DELR ${ }^{\star \star 2}$

DELZ = DELR/TAN30

$R(1)=R S$

$\mathrm{IZO}=1$

IRO = 1

IF(RS.GE.RB)THEN

$Z(1)=(R S-R B) / T A N 30+H B$
NZS $=3$

ELSE

IRO = IRO + 1

$R W 0=D E L R+R S$

2 IF(RWO.GT.RB) THEN

$Z(1)=(R W 0-R B)^{\star} T A N 30+H B$

IF(Z(1).GT.DELR) THEN

$4 \quad Z(1)=Z(1)-D E L$

$I Z 0=\mid Z O+1$

IF(Z(1).GT.DELZ) GOTO

ENDIF

$N Z S=120+1$

WRITE $(8, \star)$ 'HERE DS $\angle D B$, IRO = ',IRO GOTO 6

ENDIF

$R W O=R W O+D E L R$

IRO = IRO + 1

GOTO 2

ENDIF

6 CONTINUE

IROM = IRO - 1

IROMM $=$ IROM - 1

$I Z O M=I Z O-1$

$I Z O P=I Z O+1$

DELRZ $=$ DELR*DELZ

DELRZ2 $=(\text { DELR/DELZ })^{* * 2}$

$N Z P=1$

$8 Z 0=Z(1)+D E L Z^{*} N Z P$

$N Z P=N Z P+1$

IF(ZO.LT.H) GOTO 8

$N Z=N Z P-1$

$N Z M=N Z-1$

$N Z M M=N Z-2$

WRITE $(8, *)$ ' N,NR, NZ,ALPHA,DELR,Z1 = 'NRM,NR,NZ,ALPHA,DELR,Z(1)

WRITE $\left(8,{ }^{*}\right)$ ' DS,DB,F1,F2,H,DCOL= ',DS,DB,F1,F2,H,DCOL

$\mathrm{PMORH}=\mathrm{PMOR}(\mathrm{H})$

PMORG(1) = PMORH - PMOR(Z(1))

$R W(1)=R B+(Z(1)-H B) * T A N 30$

DO $101=2, N Z$

$Z(I)=Z(I-1)+D E L Z$

$R W(I)=R B+(Z(I)-H B) * T A N 30$

IF $(Z(I) \cdot L T . H B) R W(I)=R B$ 
IF(RW(I).GT.RO) RW(I) = RO

$\operatorname{PMORG}(I)=$ PMORH-PMOR $(Z(I))$

10 CONTINUE

DPMOR(1) = (4. *PMORG(2)-PMORG(3)-3. *PMORG(1))/2./DELR

DPMOR(NZ) $=\left(P M O R G(N Z-2)-4 .{ }^{\star} P M O R G(N Z M)+3 .{ }^{*} P M O R G(N Z)\right) / 2 . / D E L R$ DO $15 \mathrm{I}=2, \mathrm{NZM}$

DPMOR(I) $=($ PMORG $(1+1)-P M O R G(1-1)) / 2 . / D E L R$

15 CONTINUE

17 FORMAT('I,PMORG,DPMOR = ',/5G12.5)

C $\quad$ DO $181=1, N Z$

C WRITE(8,17)I,PMORG(I),DPMOR(I)

C 18 CONTINUE

C STOP

DO $20 \mathrm{~J}=2, \mathrm{NR}$

IF(J.LT.IRO)R $(J)=R(J-1)+D E L R$

IF(J.GE.IRO)R(J) = RW(IZO+J-IRO)

C WRITE $\left(8,{ }^{*}\right)^{\prime} R(J), R W, N R={ }^{\prime}, R(J), R W(J),(R(J)-R S) / D E L R$ 20 CONTINUE

DELZS = H - Z(NZ)

ZDIVS = DELZS/DELZ

WRITE $\left(8,{ }^{\star}\right)^{\prime}$ DELZS,DELZ,DELR = ', DELZS,DELZ,DELR

ZDIVSP $=1 .+$ ZDIVS

ZDIVSM $=1 .-1$. IZDIVS

DNM $=2 *(1+D E L R Z 2)$

DNMS $=2 . *(1 .+D E L R Z 2 / Z D I V S)$

$E 1=(1 .+D E L R / 2.) / D N M$

$E 2=(1 .-D E L R / 2.) / D N M$

$E 3=$ DELRZ2/DNM

$E 4=E 1 * D N M / D N M S$

$E 5=E 2 * D N M / D N M S$

E6 = 2. ${ }^{*} D E L R Z 2 / Z D I V S P / D N M S$

IF(IRO.GT.1) THEN

$D E L Z B=Z(1)$

ZDIVB $=$ DELZB/DELZ

ZDIVBP $=1 .+$ ZDIVB

IF(IRO.EQ.2)THEN

DELRB = RB - RS

ELSE

$D E L R B=R B-I R O M$ * DELR - RS

ENDIF

RDIVB = DELRB/DELR

RDIVBP $=1$. + RDIVB
RDIVBM = 1. - 1./RDIVB

ENDIF

$I I=120$

$J J=I R 0$

DO $50 \mathrm{I}=1, \mathrm{NZP}$

DO $40 \mathrm{~J}=1, \mathrm{NRP}$

$\operatorname{STR}(I, J)=0$

PERG $(I, J)=P M O R G(1)$

$\operatorname{IF}(R(J) . L T . R W(I)) S T R(I, J)=(R W(I)-R(J)) \star Z(I) \star 1 . D-3$

IF(II.EQ.I.AND.JJ.EQ.J)THEN

$\|=\|+1$

$\mathrm{J} J=\mathrm{J} J+1$

$\operatorname{STR}(I, J)=0$.

ENDIF

DO $30 K=1,5$

$\operatorname{VEL}(K, I, J)=1$

30 CONTINUE

40 CONTINUE

$\operatorname{STR}(I, N R)=0$.

50 CONTINUE

55 CONTINUE

DO 1000 ITER=1, MAXIT

DIFMAX $=0$.

*

if th

*

IF(IRO.EQ.1)THEN

DR1 = -STR(2,NRP)/2./DELR

DR1M = -STR(1,NRP)/DELR

$\mathrm{DZ1}=\operatorname{STR}(3,1) / 2 . / \mathrm{DELZ}$

DZR = (VEL $(1,3,1)-D R 1 M) / 2 . / D E L Z$

CALL FSTR(DZ1,DR1,DZR, C1,C2,C3,C4,C5,C6)

$\mathrm{CO}=\mathrm{DPMOR}(2){ }^{\star R S} /\left(\mathrm{F} 1+\mathrm{F} 2 / \mathrm{RS}^{*} \mathrm{DSQRT}\left(\mathrm{DZ} 1^{* * 2+D R} 1^{* * 2}\right)\right)$

$\mathrm{C7}=2{ }^{\circ} \mathrm{C} 1-\mathrm{C5}$

STRNEW = ALPHAM ${ }^{\star S T R}(2,1)+A L P H A *(C 5 * S T R(2,2)+$

$+\quad$ C7*STR(2,NRP)+C2*DELRZ2*

$+\quad(\operatorname{STR}(3,1)+S T R(1,1))+C 4^{*}$ DELR2)/2./C6

DIFMAX = DMAX1(DIFMAX,DABS(STRNEW-STR(2,1))

$\operatorname{STR}(2, N R P)=-C O * 2 .{ }^{*} D E L R$

$\operatorname{VEL}(1,2,1)=$ DR1 
$\operatorname{VEL}(2,2,1)=D Z 1$

$\operatorname{STR}(2,1)=$ STRNEW

$\mathrm{DZ1}=\operatorname{STR}(2,1) / \mathrm{DELZ}$

$\mathrm{CO}=\mathrm{DPMOR}(1)^{\star} \mathrm{RS} /\left(\mathrm{F} 1+\mathrm{F} 2 / \mathrm{RS}^{\star} \mathrm{DSQRT}\left(\mathrm{DZ} 1^{\star \star} 2+\mathrm{DR} 1 \mathrm{M}^{\star \star} 2\right)\right)$

$\operatorname{STR}(2, N R P)=-C 0^{*} D E L R$

- If the spout diameter is less than the cone neck

ELSEIF(IRO.EQ.2) THEN

DR1 $=-S T R(1, N R P) / 2 . / D E L R B$

$D Z 1=S T R(2,1) / 2 . / D E L Z B$

$\mathrm{DZR}=(\mathrm{STR}(2, \mathrm{NRP}) / 2 . / \mathrm{DELR}-\mathrm{DR} 1+\mathrm{DR} 1 / \mathrm{ZDIVB}) / 2 . / \mathrm{DELZ}$

CALL FSTR(DZ1,DR1,DZR,C1,C2,C3,C4,C5,C6)

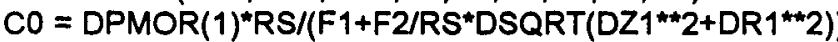

STRNEW $=$ ALPHAM ${ }^{*}$ TTR $(1,1)+A L P H A *\left(\left(C 1-C 3^{\star} D E L R B / 2 .\right)^{*}\right.$

$+\quad \operatorname{STR}(1, N R P)+2 .{ }^{*} C 2 / Z D I V B P * D E L R Z 2^{\star} R D I V B * 2^{*} \operatorname{STR}(2,1)$

$+\quad+C 4^{\star}$ DELRB $\left.{ }^{\star *} 2\right) / 2 . /\left(C 1+C 2 / Z D I V B^{\star} D E L R Z 2^{*} R D I V B^{\star \star} 2\right)$

DIFMAX = DMAX1(DIFMAX,DABS(STRNEW-STR $(2,1))$ )

$\operatorname{STR}(2, N R P)=-\mathrm{CO}^{*} 2 .{ }^{*} \mathrm{DELRB}$

$\operatorname{VEL}(1,1,1)=$ DR1

$\operatorname{VEL}(2,1,1)=D Z 1$

$\operatorname{STR}(1,1)=$ STRNEW

IF(IZO.GT.2) THEN

DO $60 \mathrm{I}=2,120 \mathrm{M}$

$\mathrm{IP}=1+1$

IM $=1-1$

DR1 $=-S T R(1, N R P) / 2 . / D E L R B$

$D Z 1=(S T R(I P, 1)-S T R(I M, 1)) / 2 . / D E L Z$

DZR = (STR(IP,NRP)-STR(IM,NRP))/4./DELRB/DELZ

CALL FSTR(DZ1,DR1,DZR,C1,C2,C3,C4,C5,C6)

$C 0=D P M O R(1) * R S /\left(F 1+F 2 / R S * D S Q R T\left(D Z 11^{* *} 2+D R 1 * * 2\right)\right)$

STRNEW $=$ ALPHAM ${ }^{\star} S T R(1,1)+A L P H A^{*}\left(\left(C 1-C 3^{\star} D E L R B / 2 .\right)^{\star}\right.$

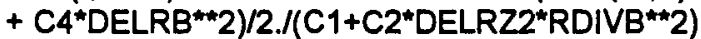

DIFMAX = DMAX 1 (DIFMAX,DABS(STRNEW-STR $(1,1))$ )

$\operatorname{STR}(I, N R P)=-C 0^{\star} 2 .{ }^{*} D E L R$

$\operatorname{VEL}(1,1,1)=\mathrm{DR} 1$

$\operatorname{VEL}(2,1,1)=D Z 1$

$\operatorname{STR}(1,1)=$ STRNEW

60 CONTINUE

ENDIF

$1=120$
$\mathbb{P}=1+1$

IM $=1-1$

DR1 $=-S T R(1, N R P) / 2 . / D E L R$

$\mathrm{DZ1}=(\mathrm{STR}(\mathrm{IP}, 1)-\mathrm{STR}(\mathrm{IM}, 1)) / 2 . / \mathrm{DELZ}$

DZR = $(V E L(1, I P, 1)-V E L(1, I M, 1)) / 2 . / D E L Z$

CALL FSTR(DZ1,DR1,DZR,C1,C2,C3,C4,C5,C6)

$\mathrm{CO}=\mathrm{DPMOR}(\mathrm{I}){ }^{\star R S} /\left(\mathrm{F} 1+\mathrm{F} 2 / \mathrm{RS}^{\star} \mathrm{DSQRT}\left(\mathrm{DZ} 1^{\star *} 2+\mathrm{DR} 1^{\star *} 2\right)\right)$

$\mathrm{C} 7=2 .{ }^{\star} \mathrm{C} 1-\mathrm{C} 5$

STRNEW = ALPHAM*STR $(1,1)+A L P H A *(C 5 * S T R(1,2)+$

$+\quad$ C7*STR(I,NRP)+C2*DELRZ2*

$\left.+\quad(S T R(I P, 1)+S T R(I M, 1))+C 4^{\star} D E L R 2\right) / 2 . / C 6$

DIFMAX = DMAX1(DIFMAX,DABS(STRNEW-STR $(1,1))$ )

$\operatorname{STR}(I, N R P)=-$ CO*2. "DELR

$\operatorname{VEL}(1,1,1)=\mathrm{DR} 1$

$\operatorname{VEL}(2, I, 1)=D Z 1$

$\operatorname{STR}(1,1)=$ STRNEW

" If the second $r$ node exist, calculate str at $I=1$

DR1 $=(\operatorname{STR}(1,2)-S T R(1, N R P)) / 2 . / D E L R$

$D Z 1=(\operatorname{STR}(2,1)-\operatorname{STR}(1,1)+\operatorname{STR}(1,1) / Z D I V B) / 2 . / D E L Z$

$D Z R=(V E L(1,2,1)-D R 1+D R 1 / Z D I V B) / 2 . / D E L Z$

CALL FSTR(DZ1,DR1,DZR,C1,C2,C3,C4,C5,C6)

$\mathrm{CO}=\mathrm{DPMOR}(1)^{\star} \mathrm{RS} /\left(\mathrm{F} 1+\mathrm{F} 2 / \mathrm{RS}^{\star} \mathrm{DSQRT}\left(\mathrm{DZ1} 1^{\star \star} 2+\mathrm{DR} 1^{\star \star *} 2\right)\right)$

$\mathrm{C} 7=2{ }^{*} \mathrm{C} 1-\mathrm{C} 5$

STRNEW $=$ ALPHAM*STR(1,1)+ALPHA* ${ }^{*} 5^{\star} S T R(1,2)+$

+ $\quad$ C7*STR(1,NRP)+2. *C2*DELRZ2/ZDIVPB*

$\left.+\quad \operatorname{STR}(2,1)+C 4^{*} D E L R 2\right) / 2 . /(C 1+C 2 * D E L R Z 2 / Z D I V B)$

DIFMAX = DMAX1(DIFMAX,DABS(STRNEW-STR $(1,1))$ )

$\operatorname{STR}(1, N R P)=\operatorname{STR}(1,2)-\operatorname{CO}{ }^{*}$. ${ }^{* D E L R}$

$\operatorname{VEL}(1,1,1)=$ DR1

$\operatorname{VEL}(2,1,1)=\mathrm{DZ1}$

$\operatorname{STR}(1,1)=$ STRNEW

IF(IROM.LE.2) GOTO 80

DO $70 \mathrm{~J}=2$,IROMM

$J P=J+1$

$J M=J-1$

DR1 $=(\operatorname{STR}(1, J P)-S T R(1, J M)) / 2 . / D E L R$

$D Z 1=(\operatorname{STR}(2, J)-S T R(1, J)+S T R(1, J) / Z D I V B) / 2 . / D E L Z$

DZR = (VEL(1,2,J)-DR1+DR1/ZDIVB)/2./DELZ

$R X=R(J)$ 
CALL FSTR(DZ1,DR1,DZR,C1,C2,C3,C4,C5,C6)

$\mathrm{C} 7=2 .{ }^{*} \mathrm{C} 1-\mathrm{C} 5$

STRNEW $=$ ALPHAM ${ }^{*} T R(1, J)+A L P H A *\left(C 5^{*} \operatorname{STR}(1, J P)+\right.$

$\left.+\quad \operatorname{STR}(2, J)+C 4^{*} D E L R 2\right) / 2 . /\left(C 1+C 2^{*} D E L R Z 2 / Z D I V B\right)$

DIFMAX = DMAX1 (DIFMAX,DABS(STRNEW-STR $(1, J))$ )

$\operatorname{VEL}(1,1, J)=D R 1$

$\operatorname{VEL}(2,1, \mathrm{~J})=\mathrm{DZ} 1$

$\operatorname{STR}(1, \mathrm{~J})=$ STRNEW

70 CONTINUE

80 CONTINUE

DR1 $=($ RDIVBM* $S T R(1, I R O M)-S T R(1, I R O M M) / 2 / D E L R$

DZ1 $=(\operatorname{STR}(2, I R O M)-S T R(1, I R O M)+S T R(1, I R O M) / Z D I V B) / 2 . / D E L Z$

$D Z R=(V E L(1,2, I R O M)-D R 1+D R 1 / Z D I V B) / 2 . J D E L Z$

$R X=R(I R O M)$

CALL FSTR(DZ1,DR1,DZR,C1,C2,C3,C4,C5,C6)

STRNEW = ALPHAM*STR $(1$, IROM $)+A L P H A^{*}\left(\left(2 .{ }^{*} C 1 / Z D I V B P+C 3^{*} D E L R B / 2.\right)+\right.$

$+\quad$ *STR(1,IROMM)+2.*C2*DELRZ2/ZDIVBP*

$+\quad \operatorname{STR}(2$, IROM)+C4*DELR2)/2./(C1/RDIVB+C2*DELRZ2/ZDIVB

$+\quad$-RDIVBM*C $3^{*}$ DELRB/4.)

DIFMAX = DMAX1(DIFMAX,DABS(STRNEW-STR(1,IROM)))

$\operatorname{VEL}(1,1, \mathrm{IROM})=\mathrm{DR} 1$

$\operatorname{VEL}(2,1, \mathrm{ROM})=\mathrm{DZ1}$

$\operatorname{STR}(1, \mathrm{IROM})=$ STRNEW

* If the second $r$ node exist, calculate str at $1>1>1 Z 0$

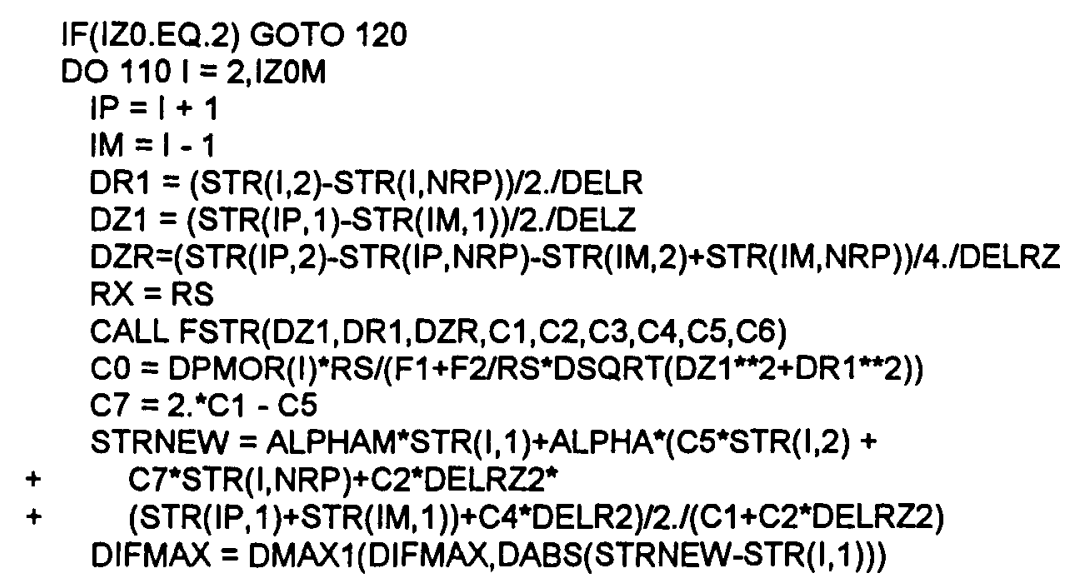




\section{$\mathrm{RX}=\mathrm{RS}$}

CALL FSTR(DZ1,DR1,DZR,C1,C2,C3,C4,C5,C6)

$\mathrm{CO}=\mathrm{DPMOR}(\mathrm{IZO})^{\star} \mathrm{RS} /\left(\mathrm{F} 1+\mathrm{F} 2 / \mathrm{RS}^{*} \mathrm{DSQRT}\left(\mathrm{DZ} 1^{* \star} 2+\mathrm{DR} 1^{\star \star} 2\right)\right)$

$\mathrm{C} 7=2{ }^{*} \mathrm{C} 1-\mathrm{C} 5$

STRNEW = ALPHAM ${ }^{\star} S T R(I Z 0,1)+A L P H A *(C 5 \star S T R(I Z O, 2)+$

$+$

$C 7^{\star}$ STR $($ IZO,NRP $)+C 2^{\star}$ DELRZ2*

$+\quad$ (STR (IZOP, 1)+STR(IZOM,1))+C4*DELR2)/2./(C1+C2*DELRZ2) DIFMAX = DMAX1(DIFMAX,DABS(STRNEW-STR(IZO,1)))

$\operatorname{STR}(I Z O, N R P)=\operatorname{STR}(I Z 0,2)-\mathrm{CO}^{\star} 2 .{ }^{*} \mathrm{DELR}$

$\operatorname{VEL}(1, \mid Z 0,1)=D R 1$

$\operatorname{VEL}(2, \mid Z 0,1)=D Z 1$

$\operatorname{STR}(I Z O, 1)=S T R N E W$

IF(IROM.LE.2) GOTO 135

DO $130 \mathrm{~J}=2, \mathrm{IROMM}$

$\mathrm{JP}=\mathrm{J}+1$

$J M=J-1$

DR1 $=(S T R(I Z O, J P)-S T R(I Z O, J M)) / 2 . / D E L R$

$D Z 1=(S T R(I Z O P, J)-S T R(I Z O M, J)) / 2 . / D E L Z$

$D Z R=(V E L(1, I Z O P, 1)-V E L(1, I Z O M, 1)) / 2 . / D E L Z$

$R X=R(J)$

CALL FSTR(DZ1,DR1,DZR,C1,C2,C3,C4,C5,C6)

$\mathrm{C} 7=2 .{ }^{*} \mathrm{C} 1-\mathrm{C} 5$

STRNEW $=$ ALPHAM ${ }^{\star} S T R(I Z O, J)+A L P H A *(C 5 * S T R(I Z O, J P)+$ $C 7^{\star} S T R(I Z O, J M)+C 2 * D E L R Z 2 *$

$\left.+\quad(S T R(I Z O P, J)+S T R(I Z O M, J))+C 4^{\star} D E L R 2\right) / 2 . /\left(C 1+C 22^{\star} D E L R Z 2\right)$ DIFMAX = DMAX $1(D I F M A X, D A B S(S T R N E W-S T R(I Z O, J)))$

$\operatorname{VEL}(1, I Z 0, J)=D R 1$

$\operatorname{VEL}(2, \mid Z 0, J)=D Z 1$

$\operatorname{STR}(I Z O, J)=$ STRNEW

130

CONTINUE

135 CONTINUE

DR1 = -STR(IZO,IROMM)/2./DELR

$D Z 1=(S T R(I Z O P, I R O M)-S T R(I Z O M, I R O M)) / 2 . / D E L Z$

DZR = (VEL(1,IZOP,IROM)-VEL(1, IZOM,IROM))/2./DELZ

$\mathrm{RX}=\mathrm{R}(\mathrm{IROM})$

CALL FSTR(DZ1,DR1,DZR,C1,C2,C3,C4,C5,C6)

$C 7=2 .{ }^{\star} \mathrm{C} 1$ - $\mathrm{C} 5$

STRNEW = ALPHAM ${ }^{\star S T R}(I Z O, I R O M)+A L P H A *\left(C 5^{\star} S T R(I Z O, I R 0)+\right.$

C7*STR(IZO,IROMM)+C2*DELRZ2*

$\left.+\quad(S T R(I Z O P, I R O M)+S T R(I Z O M, I R O))+C 4{ }^{\star} D E L R 2\right)$

$+\quad 12 . /(C 1+C 2 * D E L R Z 2)$

DIFMAX = DMAX1(DIFMAX,DABS(STRNEW-STR(IZO,IROM)))
VEL(1,IZO,IROM) = DR1

$\operatorname{VEL}(2, I Z O, I R O M)=D Z 1$

STR $(I Z O, I R O M)=$ STRNEW

ENDIF

DO 140 I = NZS,NZM

$\mathrm{IP}=1+1$

IM $=1-1$

$D Z 1=(S T R(I P, 1)-S T R(I M, 1)) / 2 . / D E L Z$

DR1 $=(\operatorname{STR}(1,2)-S T R(I, N R P)) / 2 . / D E L R$

DZR = (STR (IP,2)-STR(IM,2)-STR(IP,NRP)+STR(IM,NRP))/4./DELRZ

$\mathrm{RX}=\mathrm{RS}$

CALL FSTR(DZ1,DR1,DZR,C1,C2,C3,C4,C5,C6)

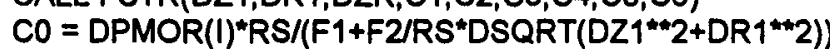

$C 7=2{ }^{\star} \mathrm{C} 1-\mathrm{C} 5$

STRNEW $=$ ALPHAM ${ }^{\star} S T R(1,1)+A L P H A * C 5^{\star}$ STR $(1,2)+C 7^{\star}$ STR $(1, N R P)$

$\left.+\quad+C 2 * D E L R Z 2 *(S T R(I P, 1)+S T R(I M, 1))+C 44^{\star} D E L R 2\right) / 2 . / C 6$

DIFMAX = DMAX1(DIFMAX,DABS(STRNEW-STR $(1,1))$ )

$\operatorname{VEL}(1, I, 1)=\mathrm{DR} 1$

VEL $(2,1,1)=D Z 1$

$\operatorname{STR}(1,1)=$ STRNEW

$\operatorname{STR}(1, N R P)=\operatorname{STR}(1,2)-\operatorname{CO}{ }^{\star} 2$. DELR

DO $137 \mathrm{~J}=2$, NRM

$J P=J+1$

$J M=J-1$

IF(RW(I).LT.RO.AND.R(JP).EQ.RW(I)) THEN

DZ1 = STR(IP,J)/2./DELZ

DR1 = -STR $(I, J M) / 2 . / D E L R$

DZR $=(\operatorname{STR}(\mathrm{I}, \mathrm{J})-\mathrm{STR}(\mathrm{IP}, \mathrm{JM})) / 4 . / D E L R Z$

$R X=R(J)$

CALL FSTR(DZ1,DR1,DZR,C1,C2,C3,C4,C5,C6)

$C 7=2 * C 1-C 5$

STRNEW = ALPHAM*STR $(I, J)+A L P H A *(C 5 * S T R(I, J P)+$

$+\quad C 7^{\star} S T R(1, J M)$

$+\quad+$ C2*DELRZ2*(STR(IP,J)+STR $(I M, J))+C 4^{*}$ DELR2)/2./C6 DIFMAX = DMAX1(DIFMAX,DABS(STRNEW-STR $(I, J))$ )

$\operatorname{VEL}(1, I, J)=$ DR1

VEL $(2, I, J)=D Z 1$

$\operatorname{STR}(I, J)=$ STRNEW

GOTO 140

ENDIF

DZ1 = (STR $(I P, J)-S T R(I M, J)) / 2 . / D E L Z$

DR1 $=(\operatorname{STR}(\mathrm{I}, \mathrm{JP})-\mathrm{STR}(\mathrm{I}, \mathrm{JM})) / 2 . / D E L R$

Appendix III: Computer Program Listings 


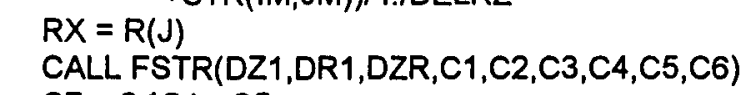

Appendix III: Computer Program Listings
CALL FSTR(DZ1,DR1,DZR,C1,C2,C3,C4,C5,C6)

$\mathrm{C} 7=2 .{ }^{\star} \mathrm{C} 1$ - $\mathrm{C} 5$

STRNEW $=$ ALPHAM ${ }^{\star} S T R(N Z, J)+A L P H A^{*}\left(C 5^{\star} S T R(N Z, J P)+\right.$

$+\quad$ C7*STR(NZ,JM)+2. ${ }^{*} \mathrm{C} 2{ }^{*}$ DELRZ2/ZDIVSP*

$+\quad$ STR(NZM J)+C4*DELR2)/2/(C1+C2*DELRZ2/ZDIVSP)

DIFMAX = DMAX1(DIFMAX,DABS(STRNEW-STR(NZ,J)))

$\operatorname{VEL}(1, N Z, J)=D R 1$

VEL(2,NZ,J) $=D Z 1$

$\operatorname{STR}(N Z, J)=$ STRNEW

$\operatorname{STR}(N Z P, J)=S T R(N Z, J)$

IF(RW(NZ).LT.RO.AND.R(JP).EQ.RW(NZ))GOTO 160

150 CONTINUE

160 CONTINUE

C IF(ITER/100*100.EQ.ITER)WRITE(8,170)ITER,STR(NZ,1), STR(NZ,5)

$\mathrm{C}+, \mathrm{STR}(\mathrm{NZ}, 10), \mathrm{STR}(\mathrm{NZ}, 15), \mathrm{STR}(\mathrm{NZ}, \mathrm{NR})$,DIFMAX

170 FORMAT(I5,6G12.3)

IF (DIFMAX.LT.EPS)GOTO 2000

1000 CONTINUE

WRITE $\left(6,{ }^{*}\right)$ ' Convergence Failure - DIFMAX = ',DIFMAX

2000 IF(ILIN.EQ.0)THEN

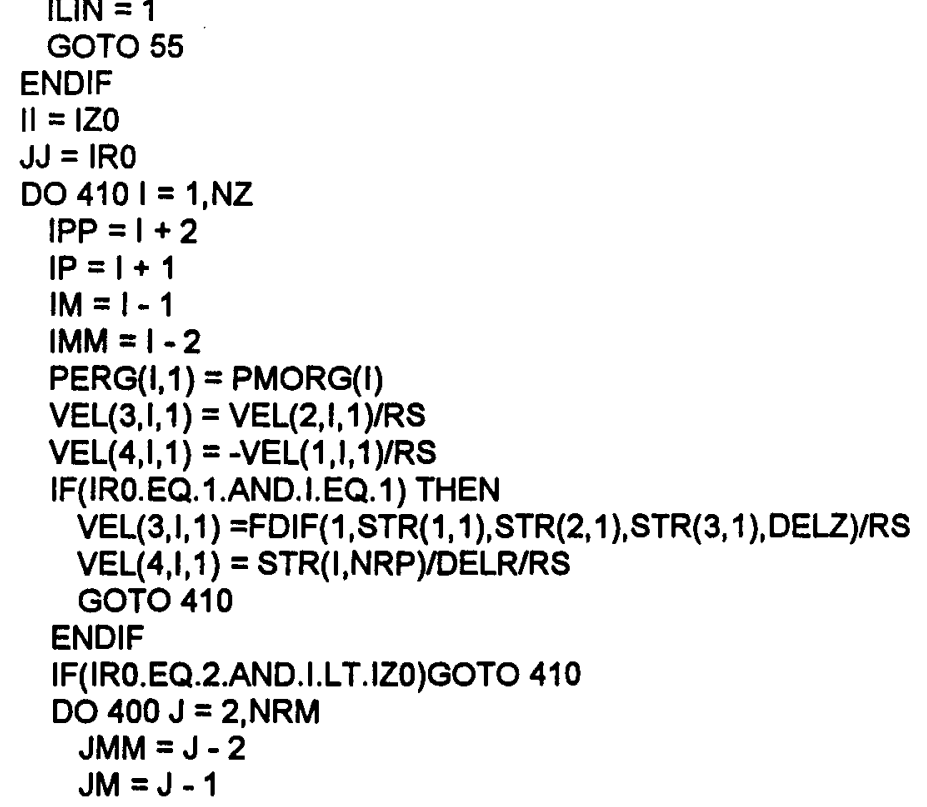


IF (JMM.EQ.0) JMM = NRP

IF(IR0.EQ.1.AND.J.EQ.2.AND.I.EQ.J) THEN

$\operatorname{VEL}(3, I, J)=\operatorname{FDIF}(1, \operatorname{STR}(I, J), \operatorname{STR}(I P, J), \operatorname{STR}(I P P, J), D E L Z)$

$$
/ R(J)
$$

$\operatorname{VEL}(4, I, J)=-\operatorname{FDIF}(3, \operatorname{STR}(1, J), \operatorname{STR}(I, 1), \operatorname{STR}(I, N R P), D E L R)$

$+\quad \operatorname{RR}(\mathrm{J})$

$\|=\|+2$

$\mathrm{J} J=\mathrm{J} J+2$

GOTO 410

ELSEIF(II.EQ.I.AND.JJ.EQ.J) THEN

$\operatorname{VEL}(3, I, J)=F D I F(1, \operatorname{STR}(I, J), \operatorname{STR}(I P, J), \operatorname{STR}(I P P, J), D E L Z)$

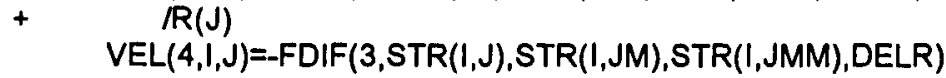

$+\quad \operatorname{lR}(\mathrm{J})$

$\|=\|+1$

$\mathrm{JJ}=\mathrm{JJ}+1$

GOTO 410

ELSE

$\operatorname{VEL}(3, I, J)=\operatorname{VEL}(2, I, J) / R(J)$

$\operatorname{VEL}(4,1, J)=-\operatorname{VEL}(1, I, J) / R(J)$

IF(I.LT.II.AND.J+1.EQ.JJ) GOTO 410

$$
\text { ENDIF }
$$

400 CONTINUE

$$
\operatorname{VEL}(3, I, N R)=0 \text {. }
$$

VEL $(4, I, N R)=-F D I F(3, S T R(I, N R), S T R(I, N R M), S T R(I, N R M M), D E L R)$

$$
+\quad \text { IR(NR) }
$$

410 CONTINUE

$$
I I=I Z 0
$$

$\mathrm{JJ}=\mathrm{IRO}$

MJUN $=0$

WRITE $(8, *) ' N Z, N R=$ ',NZ,NR

DO $4301=1, N Z$

$I P P=1+2$

$I P=1+1$

$I M=1-1$

IMM $=1-2$

IF(I.EQ.1.AND.IRO.EQ.1) GOTO 430

IF(I.LT.IZO.AND.IRO.EQ.2) GOTO 430

DO $420 \mathrm{~J}=2$, NRM

$\mathrm{JMM}=\mathrm{J}-2$

$J M=J-1$

IF(JMM.EQ.0) JMM = NRP
UAR = VEL $(3,1, J)$

$U Z=V E L(4, I, J)$

IF(UAR+UZ.LE.0.)WRITE(8,*)'ZERO AT*',1,J,NZ

$J P=J+1$

IF(I.EQ.NZ) THEN

DURR = FDIF(2,VEL(3,1,JP),VEL $(3,1, J M), X X, D E L R)$

DUZR = FDIF $(2, \operatorname{VEL}(4,1, J P), V E L(4,1, J M), X X, D E L R)$

DURZ $=\left(\right.$ ZDIVSM ${ }^{*}$ VEL $\left.(3,1, J)-V E L(3, I M, J)\right) / 2 . / D E L Z$

DUZZ $=(\operatorname{VEL}(4,1, J)-\operatorname{VEL}(4, I M, J)) / 2$. IDELZ

VEL $(5,1, J)=F 2^{\star}\left(U^{*}{ }^{* *} 2^{*}\right.$ UURR+UAR*UZ*(DUZR+DURZ)

$+\quad$ +UZ**2*DUZZ/ DSQRT(UAR**2+UZ**2)

ELSEIF(IRO.EQ.1.AND.J.EQ.2.AND.I.EQ.J) THEN

DURR $=(\operatorname{VEL}(3,1, J)-V E L(3,1, J M)) / D E L R$

DURZ = FDIF $(1, \operatorname{VEL}(3, I, J), \operatorname{VEL}(3, I P, J), V E L(3, I P P, J), D E L Z)$

DUZR $=$ (VEL $(4, I, J)-V E L(4, I, J M)) / D E L R$

DUZZ = FDIF $(1, \operatorname{VEL}(4, I, J), \operatorname{VEL}(4, \operatorname{IP}, J), \operatorname{VEL}(4, I P P, J), D E L Z)$

VEL $(5, I, J)=F 2^{*}\left(\right.$ UAR ${ }^{* *} 2^{*}$ DURR+UAR*UZ*(DUZR+DURZ)

$+\quad+U Z^{\star \star} 2^{\star}$ DUZZ)/ DSQRT(UAR $\left.{ }^{\star \star} 2+U Z^{\star *} 2\right)$

$\|11=\|+2$

$\mathrm{J}=\mathrm{JJ}+2$

GOTO 430

ELSEIF(II.EQ.I.AND.JJ.EQ.J) THEN

DURR = FDIF $(3, \operatorname{VEL}(3,1, J), \operatorname{VEL}(3,1, J M), \operatorname{VEL}(3,1, J M M), D E L R)$

DURZ = FDIF $(1, \operatorname{VEL}(3,1, J), \operatorname{VEL}(3, I P, J), \operatorname{VEL}(3, I P P, J), D E L Z)$

DUZR = FDIF(3,VEL(4,I,J), VEL(4,I,JM), VEL(4,I,JMM),DELR)

DUZZ = FDIF(1,VEL(4,I,J),VEL(4,IP,J),VEL $(4, I P P, J), D E L Z)$

IF(J.EQ.2.AND.IRO.EQ.2) THEN

DURR $=($ VEL $(3,1, J)-V E L(3,1, J M)) / D E L R$

DUZR $=(\operatorname{VEL}(4, I, J)-V E L(4, I, J M)) / D E L R$

\section{ENDIF}

$\operatorname{VEL}(5, I, J)=F 2 *\left(U A R * 2^{*}\right.$ DURR+UAR*UZ*(DUZR+DURZ)

$+U Z^{*+2 * D U Z Z) / ~ D S Q R T(U A R * * 2+U Z * * 2) ~}$

$\|=\|+1$

$J J=J J+1$

GOTO 430

ELSE

DURR = FDIF(2,VEL(3,I,JP), VEL(3,I,JM),XX,DELR

DURZ = FDIF (2 VEL $(3, I P, J)$ VEL $(3, I M, J), X X, D E L Z)$

DUZR = FDIF $(2, \operatorname{VEL}(4,1, J P), \operatorname{VEL}(4,1, J M), X X, D E L R)$

DUZZ = FDIF $(2, V E L(4, I P, J), V E L(4, I M, J), X X, D E L Z)$

VEL $(5, I, J)=F 2 *(U A R * 2 * D U R R+U A R * U Z *(D U Z R+D U R Z)$ $\left.+U Z^{* *} 2 * D U Z Z\right) /$ DSQRT(UAR**2+UZ**2)

Appendix III: Computer Program Listings 
IF(I.LT.II.AND.J+1.EQ.JJ) GOTO 430

ENDIF

420 CONTINUE

$\mathrm{UAR}=\mathrm{VEL}(3, \mathrm{I}, \mathrm{NR})$

$U Z=V E L(4, I, N R)$

IF(UAR+UZ.LE.0.)WRITE(8, $\left.{ }^{*}\right)$ 'ZERO AT ',I,NR

IF(MJUN.EQ.0)THEN

DURR = FDIF(3,VEL(3,I,NR),VEL(3,I,NRM),VEL(3,I,NRMM),DELR)

DURZ = FDIF(1,VEL(3,I,NR),VEL(3,IP,NR), VEL(3,IPP,NR),DELZ)

DUZR = FDIF $(3, V E L(4, I, N R), V E L(4, I, N R M), V E L(4, I, N R M M), D E L R)$

DUZZ = FDIF(1,VEL(4,I,NR),VEL(4,IP,NR),VEL(4,IPP,NR),DELZ)

VEL $(5, I, N R)=F 2^{*}\left(U A R^{\star \star} 2 * D U R R+U A R^{*} U Z^{\star}(D U Z R+D U R Z)\right.$

$$
+Z^{* *} 2^{\star} \text { DUZZ)/ DSQRT(UAR }{ }^{\star \star} 2+U Z^{* *} 2 \text { ) }
$$

MJUN $=1$

ELSEIF(I.EQ.NZ) THEN

DURR = FDIF(3,VEL(3,I,NR), VEL(3,I,NRM), VEL(3,I,NRMM),DELR) DURZ = FDIF $(3, \operatorname{VEL}(3, I, N R), V E L(3, I M, N R), V E L(3, I M M, N R), D E L Z)$

DUZR = FDIF (3,VEL(4,I,NR),VEL (4,I,NRM), VEL (4,I,NRMM),DELR)

DUZZ = FDIF(3,VEL(4,I,NR),VEL(4,IM,NR),VEL(4,IMM,NR),DELZ)

VEL $(5, I, N R)=F 2^{\star}\left(U A R^{\star \star} 2^{*} D U R R+U A R^{\star} U Z^{\star}(D U Z R+D U R Z)\right.$ $\left.+U Z^{\star \star} 2^{\star} D U Z Z\right) / D S Q R T\left(U A R^{\star \star} 2+U Z^{\star \star} 2\right)$

ELSE

DURR = FDIF(3,VEL(3,I,NR),VEL(3,I,NRM), VEL(3,I,NRMM),DELR)

DURZ = FDIF(1,VEL(3,IP,NR),VEL(3,IM,NR),XX,DELZ)

DUZR = FDIF $(3, V E L(4, I, N R), V E L(4, I, N R M), V E L(4, I, N R M M), D E L R)$

DUZZ = FDIF $(1, V E L(4, I P, N R), V E L(4, I M, N R), X X, D E L Z)$

VEL(5,I,NR)=F2*(UAR ${ }^{\star *} 2^{\star} D U R R+U A R * U Z^{*}$ (DUZR+DURZ)

$$
\text { ENDIF }
$$$$
\left.+Z^{* *} 2^{*} \text { DUZZ)/ DSQRT(UAR }{ }^{\star \star} 2+U Z^{\star \star} 2\right)
$$

430 CONTINUE

EPSP $=$ EPS*PERG $(1,1)$

WRITE $\left(8,{ }^{*}\right)^{\prime} E P S$, EPSP $=$ ',EPS,EPSP

ANG $=1.16$.

RDIVC $=2 .{ }^{*} D \operatorname{COS}(\mathrm{ANG})^{\star *} 2-1$.

ZDIVC $=2 .{ }^{\star}$ DCOS(ANG) ${ }^{\star}$ DSIN(ANG) ${ }^{\star}$ TAN30

DO 7000 ITER $=1$, MAXIT

II $=120$

$\mathrm{JJ}=\mathrm{IRO}$

DIFMAX $=0$.

DO $4501=1, \mathrm{NZ}$

$\mathrm{IP}=1+1$

IM $=1-1$
IF(I.EQ.1.AND.IRO.EQ.1) GOTO 450

IF(I.LT.IZO.AND.IRO.EQ.2) GOTO 450

DO $440 \mathrm{~J}=2$, NR

$\mathrm{JM}=\mathrm{J}-1$

$\mathrm{JP}=\mathrm{J}+1$

IF(I.EQ.NZ) THEN

$E 4=(1 .+D E L R / 2 . / R(J)) / D N M S$

$E 5=(1 .-D E L R / 2 / R(J)) / D N M S$

PERNEW=PERG $(I, J)^{\star A L P 2 M+A L P 2 *}{ }^{\star}\left(E 4^{*} P E R G(I, J P)+\right.$

$+\quad$ E5*PERG $(\mathrm{I}, \mathrm{JM})+E 6 * P E R G(I M, J)+V E L(5, I, J)^{\star D E L L R 2 / D N M S) ~}$

DIFMAX = DMAX1(DIFMAX,DABS(PERNEW-PERG $(1, J)))$

PERG $(I, J)=$ PERNEW

IF(II.EQ.I.AND.JJ.EQ.J) PERG(I,JP) $=0$

ELSE

$E 1=(1 .+D E L R / 2 . / R(J)) / D N M$

$E 2=(1 .-D E L R / 2 . / R(J)) / D N M$

PERNEW=PERG $(1, J)^{*} A L P 2 M+A L P 2 *(E 1 * P E R G(I, J P)+$

E2*PERG(I,JM)+E3*(PERG(IM,J)+PERG(IP,J))

$+V E L(5, I, J) * D E L R 2 / D N M)$

DIFMAX = DMAX1(DIFMAX,DABS(PERNEW-PERG(I,J)))

PERG $(I, J)=$ PERNEW

ENDIF

IF(IRO.EQ.1.AND.J.EQ.2.AND.I.EQ.J) THEN

$\mathrm{P} 2$ = $\mathrm{PERG}(\mathrm{I}, \mathrm{JM})$

$P 1=P E R G(I M, J M)$

PERG $(I M, J)=P 1+(P 2-P 1) * Z D I V C$

$\|=\|+2$

$J \mathrm{~J}=\mathrm{J} J+5$

GOTO 450

ELSEIF(II.EQ.I.AND.JJ.EQ.J) THEN

IF(J.GT.2) THEN

$J M M=J-2$

$P 1=P E R G(I M, J M)+(P E R G(I M, J M M)-P E R G(I M, J M))^{\star} R D I V C$

$P 2=P E R G(1, J M)+(P E R G(1, J M M)-P E R G(1, J M))^{\star} R D I V C$

PERG $(I M, J)=P 1+(P 2-P 1)^{\star} Z D I V C$

ELSE

P2 = PERG $(1, J M)$

P1 = PERG $(\mid \mathrm{M}, J M)$

PERG $(I M, J)=P 1+(P 2-P 1)^{\star} Z$ ZDIVC

ENDIF

IF(J.EQ.NR) PERG(I,NRP) = PERG(I,NRM)

$\|=\|+1$

Appendix III: Computer Program Listings 


$$
\mathrm{JJ}=\mathrm{JJ}+1
$$

GOTO 450

ELSEIF(I.LT.II.AND.JP.EQ.JJ)THEN

PERG $(l, J P)=P E R G(I, J)$

GOTO 450

$$
\text { ENDIF }
$$

440 CONTINUE

$$
\text { PERG }(I, N R P)=\text { PERG }(1, N R M)
$$

450 CONTINUE

C IF(ITER/10*10.EQ.ITER)WRITE $(8,170)$ ITER,PERG(NZ,1),PERG(NZ,5)

$C+$,PERG(NZ, 10),PERG(NZ, 15),PERG(NZ,NR), DIFMAX IF (DIFMAX.LT.EPSP)GOTO 8000

7000 CONTINUE

WRITE $\left(6,{ }^{\star}\right)$ ' CONVERGENCE FAILURE $\|$ - DIFMAX = ',DIFMAX

8000 CONTINUE

$N S P=N Z P$

$N S P M=N S P-1$

$D D X=(H-Z(1)) /(N S P-1$.

$J J=\mid R O$

DO $510 I=1, N Z$

$\mathrm{IP}=1+1$

IF(I.EQ.1.AND.IRO.EQ.1) THEN

$Q Z A(I)=0$.

$\mathrm{JJ}=2$

GOTO 510

ELSEIF(I.LT.IZO.AND.IRO.EQ 2) THEN

$Q Z A(I)=P I^{*} V E L(4, I, J) *(R S+R B) / 2 .{ }^{*}(R B-R S)$

GOTO 510

ENDIF

$\mathrm{QZA}(\mathrm{I})=0$.

DO $500 \mathrm{~J}=1, \mathrm{JJ}-1$

$J P=J+1$

$Q Z A(I)=Q Z A(I)+P I^{\star}\left(V E L(4, I, J) * R(J)+V E L(4, I, J P)^{\star} R(J P)\right) / 2 .{ }^{*} D E L R$

500 CONTINUE

$J \mathrm{~J}=\mathrm{JJ}+1$

IF(JJ.GT.NR) JJ $=$ NR

510 CONTINUE

CALL MYSPLN(Z,QZA,QZAQ,QZAR,QZAS,NZ,NDS)

$C Z(1)=Z(1)$

$\operatorname{PAN}(1)=\operatorname{PMORG}(1)$

$\operatorname{QAN}(1)=0$.
WRITE $(8, *)^{\prime} \mathrm{DDX}=$ ',DDX

DO $4901=2$, NSPM

$C Z(I)=C Z(I-1)+D D X$

$\operatorname{PAN}(1)=$ PMORH - PMOR(CZ(1))

QAN $(I)=$ QAINT $(C Z(I), 11)$

490 CONTINUE

$C Z(N S P)=H$

$P A N(N S P)=0$

QAN $(N S P)=Q Z A(N Z)$

$\operatorname{DPAN}(1)=$ (4."PAN(2)-PAN(3)-3."PAN(1))/2./DELR

DPAN(NSP) $=\left(\right.$ PAN(NSP-2)-4. ${ }^{*}$ PAN(NSPM)+3. ${ }^{* P A N(N S P)) / 2 . / D E L R ~}$

$\operatorname{DQAN}(1)=\left(4 .{ }^{*}\right.$ QAN(2)-QAN(3)-3.*QAN(1))/2./DELR

DQAN(NSP) $=($ QAN(NSP-2)-4."QAN(NSPM)+3. *QAN(NSP) $) / 2 . / D E L R$ DO $5201=2$, NSPM

DPAN $(I)=($ PAN $(I+1)-P A N(I-1)) / 2 . / D E L R$

DQAN $(1)=($ QAN $(1+1)-$ QAN $(1-1)) / 2 . / D E L R$

520 CONTINUE

CALL MYSPLN(CZ,QAN, QANQ, QANR, QANS, NSP,NDS)

CALL MYSPLN(CZ,DQAN,DQANQ,DQANR,DQANS,NSP,NDS)

CALL MYSPLN(CZ,PAN,PANQ,PANR,PANS,NSP,NDS)

CALL MYSPLN(CZ,DPAN,DPANQ,DPANR,DPANS,NSP,NDS)

DO $6040 \mathrm{I}=1, \mathrm{NZ}$

DO $6030 \mathrm{~J}=1, \mathrm{NR}$

$\operatorname{PLX}(J, I)=\operatorname{PERG}(I, J)$

6030 CONTINUE

6040 CONTINUE

RETURN

1111 WRITE $(8,5000)((\operatorname{STR}(I, J), J=1, N R, 2), I=1, N Z)$

C WRITE $(8,5000)((P E R G(1, J), J=1, N R, 2), l=1, N Z)$

C WRITE $(8,5000)($ VEL $(1, I, J), J=1, N R, 2), I=1, N Z)$

C WRITE $(8,5000)($ VEL $(2, I, J), J=1, N R, 2), 1=1, N Z)$

C WRITE $(8,5000)((\operatorname{VEL}(3, I, J), J=1, N R, 2), I=1, N Z)$

C WRITE $(8,5000)($ VEL $(4, I, J), J=1, N R, 2), I=1, N Z)$

C WRITE $(8,5000)((\operatorname{VEL}(5, I, J), J=1, N R, 2), l=1, N Z)$

\section{$\mathrm{RSIZ}=3.0$}

$Z S I Z=(Z(N Z)-Z(1))^{\star 2} .5 /(R O-R S)+0.5$

$X 1=0.02$

$X S T E P=0.02$

$X 2=R(N R)$

$Y 1=$ DNINT(100."Z(1))/100.

YSTEP $=0.04$ 
$\mathrm{Y} 2=\mathrm{Z}(\mathrm{NZ})$

IF(RO.GT.0.121) THEN

$\mathrm{RS} I \mathrm{Z}=3.5$

$Z S I Z=(Z(N Z)-Z(1)) * 3.0 /(R 0-R S)+0.5$

$X S T E P=0.04$

$$
\text { ENDIF }
$$

5000 FORMAT(11G12.3)

CALL DSPDEV('PLOT')

CALL AREA2D(RSIZ,ZSIZ)

CALL COMPLX

CALL XNAME('Radius, m',1)

CALL YNAME('Height, $\left.m^{\prime}, 1\right)$

CALL GRAF(X1,XSTEP,X2,Y1,YSTEP,Y2)

CALL FRAME

CALL BCOMON(50000)

DO $5020 \mathrm{I}=1, \mathrm{NZ}$

DO $5010 \mathrm{~J}=1, \mathrm{NR}$

$\operatorname{PLX}(J, I)=\operatorname{STR}(1, J)^{\star 1}$.E5

5010 CONTINUE

5020 CONTINUE

CALL CONMAK(PLX,NR,NZ,'SCALE')

CALL CONLIN $(0$, 'SOLID','LABEL',2,5)

CALL CONLIN $(1$, 'DASH','NOLABELS', 1,3)

CALL CONANG(90.)

CALL RASPLN(0.25)

CALL CONTUR(2,'LABELS', 'DRAW')

CALL ENDPL(O)

CALL DONEPL

CALL DSPDEV('PLOT')

CALL AREA2D(RSIZ,ZSIZ)

CALL COMPLX

CALL XNAME('Radius, $m$ ', 1 )

CALL YNAME('Height, $m$ ', 1)

CALL GRAF(X1,XSTEP,X2,Y1,YSTEP,Y2)

CALL FRAME

CALL BCOMON $(50000)$

DO $5040 \mathrm{I}=1, \mathrm{NZ}$

DO $5030 \mathrm{~J}=1, \mathrm{NR}$

$\operatorname{PLX}(J, I)=\operatorname{PERG}(1, J)$

5030 CONTINUE

5040 CONTINUE

CALL CONMAK(PLX,NR,NZ,'SCALE')
CALL CONLIN ( 0 ,'SOLID' 'LABEL', 2,5)

CALL CONLIN (1,'DASH', 'NOLABELS', 1,3)

CALL CONANG(90.)

CALL RASPLN $(0.25)$

CALL CONTUR(2,'LABELS','DRAW')

CALL ENDPL(O)

CALL DONEPL

END 
SUBROUTINE FSTR(DZ1,DR1,DZR,C1,C2,C3,C4,C5,C6)

IMPLICIT REAL*8(A-H,O-Z)

COMMON /FSTRSB/ F1,F2,RX,DELR,DELRZ2, ILIN

IF(ILIN.EQ.1)THEN

A2STR $=\mathrm{DZ1} 1^{\star \star 2} 2+\mathrm{DR} 1{ }^{*} 2$

IF(A2STR.LE.0.)WRITE $\left(8,{ }^{*}\right)$ ' YEP, A2STR = ',A2STR

A1STR = DSQRT(A2STR)

$\mathrm{C} 1=\mathrm{F} 1{ }^{\star} \mathrm{RX} / \mathrm{F} 2^{\star} \mathrm{A} 1 \mathrm{STR}+\mathrm{A} 2 \mathrm{STR}+\mathrm{DR} 1^{\star \star 2}$

$C 2=C 1-D R 1^{* \star 2} 2+D Z 1 * \star 2$

$C 3=-\left(F 1 / F 2+2 . / R X^{* A 1 S T R}\right)^{\star A} A 1 S T R$

$C 4=2 .{ }^{\star} D Z R{ }^{\star} D R 1{ }^{\star} D Z 1$

$C 5=C 1+C 3^{*} D E L R / 2$

$C 6=C 1+C 2 * D E L R Z 2$

RETURN

ENDIF

$\mathrm{C} 1=10$.

$C 2=10$.

$\mathrm{C} 3=1$

$\mathrm{C} 4=1$

$C 5=C 1+C 3^{\star} D E L R / 2$

$C 6=C 1+C 2 * D E L R Z 2$

END

Appendix III: Computer Program Listings
DOUBLE PRECISION FUNCTION FDIF(K,X,Y,Z,DEL)

* $\mathrm{K}: 1$ = FORWARD 2 = CENTRAL 3 = BACKWARD

IMPLICIT REAL"8(A-H,O-Z)

IF(K.EQ.1) THEN

FDIF $=(-3 . * X+4 . * Y-Z) / 2 . / D E L$

ELSEIF(K.EQ.2) THEN

FDIF $=(X-Y) / 2 . / D E L$

ELSEIF(K.EQ 3) THEN

FDIF $=\left(3 .{ }^{*} X-4 .{ }^{*} Y+Z\right) / 2 . / D E L$

ELSE

WRITE(8,*)' Error in FDIF code, code $=$ ', $K$

ENDIF

END 
DOUBLE PRECISION FUNCTION QAERG(X)

IMPLICIT REAL*8(A-H,O-Z)

INCLUDE 'H.COM1'

QAERG $=P \mid{ }^{*} Q A I N T(X, 11)^{*}$ QAINT $(X, 12)$

END

\section{SUBROUTINE PLOTMT}

IMPLICIT REAL*8(A-H,O-Z)

EXTERNAL SS

INCLUDE 'H.COM1'

INCLUDE 'H.COM4'

DIMENSION STOT(10000)

REAL XP1(10000),PR1(10000),XP2(100)

$\mathrm{N}=\mathrm{NSP}$

VAH $=V S(N) *(1 .-E S(N)) /((D C O L D S) * 2-1) /.(1 .-V O I D)$

$\operatorname{DMT}(\mathrm{N})=\mathrm{DCOL}$

$Z M T(N)=C Z(1)$

$\operatorname{DMT}(1)=\mathrm{DS}$

$\mathrm{ZMT}(1)=\mathrm{H}$

DO $101=2, \mathrm{~N}-1$

$J=1-1$

$\mathrm{ZMT}(\mathrm{N}-\mathrm{J})=\mathrm{CZ}(\mathrm{I})$

$X X X=D_{C O L}^{* *} 2-D S^{* *} 2^{*} V S(I) N A H^{*}(1 .-E S(I)) /(1 .-V O I D)$

IF(XXX.LE.0)WRITE(8,")'XXX,CZ(I),VS(I),VAH,ES(I)'

$+\quad X X X, C Z(I), V S(I), V A H, E S(I)$

$\mathrm{DMT}(\mathrm{N}-\mathrm{J})=\mathrm{DS}$

IF(XXX.GT.0.) DMT(N-J) = DSQRT $(X X X)$

IF(DMT(N-J).LE.DS) DMT(N-J) = DS+0.001* $(H-Z M T(N-J)) / H$

10 CONTINUE

WRITE $\left(8,{ }^{*}\right) ' Z M T$ NOT OK

CALL MYSPLN(DMT,ZMT,ZMTQ,ZMTR,ZMTS,N,NDS)

WRITE(8,")'ZMT OK'

$\operatorname{SMT}(1)=0$.

DO $201=2, N$

$\operatorname{SMT}(I)=0$.

IF(ZMT(I-1).GT.HCOAT) CALL NC4AD(SS,ZMT(I),ZMT(I-1), $0.5 \mathrm{D}-1, \mathrm{SMT}(\mathrm{I}), \mathrm{NFC})$

SMT $(I)=$ SMT $(1-1)+$ SMT $(1)$

C WRITE(7,")I, DMT(I), ZMT(I), SMT(I)

20 CONTINUE

C WRITE(7,*)1, DMT(1), ZMT(1), SMT(1)

C CALL MYSPLN(DMT,SMT,SMTQ,SMTR,SMTS,N,NDS)

C

* Randomly choose particle landing position and start the

* simulation UWT = PI/6. "DPU**3*RHOU 
SURE $=W S^{*} U W T M U$

VEXIT $=W U /(1 .-X S) / R H O P$

$V T O T=V S(N)^{*}(1 .-E S(N))^{*} P \mid 4^{*} D S^{\star \star 2} 2$

VCIRC $=$ VTOT - VEXIT

PEXIT = VEXITNTOT

PTOT $=$ VTOTNCIRC

WRITE $(7,8)$ SURE, VCIRC, VEXIT,PEXIT,PTOT

8 FORMAT(' SURE,VCIRC,VEXIT,PEXIT,PTOT = ',5G12.5)

$M M M=1000$

LFEED $=1$

$C 1=D S^{* * 2}$

CALL DSPDEV('PLOT')

CALL ATRANS

CALL NOBRDR

CALL COMPLX

CALL AREA2O $(5.5,5$.

CALL XNAME('Sulfur Content\$',100)

CALL YNAME('Cumulative Probability\$',100)

CALL XREVTK

CALL YREVTK

C CALL HEADIN('Sulfur Content Distribution\$',-100,3,1)

CALL YAXANG $(0$.

CALL GRAF $(0.0 .1,8,0.0,20,1$.

CALL THKCRV(3)

CALL SCLPIC(2.)

47 CONTINUE

IF(LFEED.EQ.1) THEN

DFEED $=$ DCOL-0.06

$\mathrm{CB} 1=\mathrm{DFEED} * * 2$

$\mathrm{CC}=\mathrm{DCOL}{ }^{\star \star 2} 2-\mathrm{CB} 1$

$\mathrm{C} 2=\mathrm{CB} 1-\mathrm{C} 1$

$\mathrm{C} 3=\mathrm{DCOL} * * 2-\mathrm{C} 1$

XFEED $=$ WU/RHOUNTOT ${ }^{*} \mathrm{C} 3 / \mathrm{CC}$

IF (XFEED.GT.1.) THEN

WRITE $\left(8,{ }^{*}\right)$ 'XFEED too large, inc. feed area, XF=',XFEED

STOP

ENDIF

$C A=C 2+(1 .-X F E E D) * C C$

$\mathrm{CB}=\mathrm{CA}(1$ 1. $\mathrm{XFEED})$

$\mathrm{CB} 2=-\mathrm{C} 2 /(1-\mathrm{XFEED})$

$X F M=C 2 /\left(C 2+(1 .-X F E E D){ }^{*} C C\right)$

WRITE $(8,113) \mathrm{C1}, \mathrm{C} 2, \mathrm{C} 3, \mathrm{CA}, \mathrm{CB}, \mathrm{CB} 1, \mathrm{CB} 2, \mathrm{DFEED}, \mathrm{XFEED}, \mathrm{XFM}$
113 FORMAT('C1,C2,C3,CA,CB, CB1,CB2,DFEED,XFEED,XFM = ' $+\quad 5$ G12.5/5G12.5)

ELSEIF(LFEED.EQ.0) THEN

$C=D C O L * \star 2-C 1$

ELSE

WRITE $\left(8,{ }^{\star}\right)$ ' Wrong Feed Code, LFEED = ',LFEED

STOP

ENDIF

WRITE $\left(7,{ }^{*}\right)$ 'XFM, XFEED, DFEED = ', XFM,XFEED, DFEED

XRAND $=$ RAND $(831$.)

DO $39011=1$ MMM

XRAND $=$ FRAND $(1.0)$

XRAND $=$ XRAND*PTOT

IF(LFEED.EQ.1) THEN

$D M T I=D S Q R T(C B 1+X R A N D * C C)$

ELSE

DMTI = DSQRT $(C 1+$ XRAND*C)

ENDIF

$\|=$ INTT(DMTI,DMT,NSP,DX)

STOT $(I 1)=S M T(I I)+(S M T(I I+1)-S M T(I I)) * D X /(D M T(I 1+1)-D M T(I I))$

C $\quad$ STOT $(11)=$ QAINT(DMTI, 2$)$

C WRITE(8,71)XRAND,DMTI,STOT(I1)

C IF(STOT(I1).LT.0.) STOT(I1) $=0$.

391 XRAND $=$ FRAND $(1.0)$

XRAND $=$ XRAND * PTOT

IF(XRAND.GT.1.0)GOTO 390

IF(LFEED.EQ.0) THEN

$D M T I=D S Q R T(C 1+X R A N D * C)$

ELSE

IF(XRAND.LE.XFM) THEN

DMTI = DSQRT $(C 1+X R A N D * C A)$

ELSE

$\mathrm{DMTI}=\mathrm{DSQRT}\left(\mathrm{CB} 1+\mathrm{CB} 2+\mathrm{XRAND}{ }^{\star} \mathrm{CB}\right)$

ENDIF

ENDIF

II = INTT(DMTI,DMT,NSP,DX)

$S M T I=S M T(I I)+(S M T(I I+1)-S M T(I I))^{\star D X X}(D M T(I I+1)-D M T(I I))$

C $\quad$ SMTI $=$ QAINT(DMTI,2)

C WRITE(8,71)XRAND,DMTI,STOT(I1)

C 71 FORMAT('XRAND,DMTI,STOT = ',3G12.5)

C IF(SMTI.LT.O.) SMTI $=0$.

STOT $(11)=$ STOT $(11)+$ SMTI

Appendix III: Computer Program Listings 
GOTO 391

390 CONTINUE

SUM1 $=0$.

SUMUD $=0$.

C WRITE( $\left(7,{ }^{\star}\right)^{\prime} l$, STOT, XP1 :: UWT = ',UWT

DO $55711=1$, MMM

SUM1 = SUM1 +STOT(11)

$X P 1(11)=$ STOT $(11) /($ STOT $(11)+$ UWT $)$

C WRITE(7,*)|1, STOT(I1),XP1(11)

557 CONTINUE

SAVG $=$ SUM1/(SUM1+UWT*MMM)

SUM2 $=0$.

DO $395 \mathrm{I}=1, \mathrm{MMM}$

SUM2 = SUM2 + (SAVG-XP1(I) $)^{\star \star 2}$

$X P 1(1)=$ STOT $(I) /(S T O T(1)+U W T)^{\star} X S / S A V G$

TANHC $=(0.19823-X P 1(I)) / X P 1(1) /(0.715183-X P 1(1))$

PARTUD $=($ DTANH $($ TANHC $)+1) /$.

IF(XP1(I).GT.0.715183) PARTUD $=0$

SUMUD = SUMUD + PARTUD

395 CONTINUE

UDC = SUMUD/MMM

XUDC $=U D C^{*}(1 .-X S)$

STD $=(\text { SUM2 } /(M M M-1))^{\star \star} 5$

CALL CUMP(MMM,XP1,PR1,UWT)

DO $3971=1,50$

PRINT(3,NPRINT,I) $=X P 1\left(\left.1\right|^{\star 20}\right)$

397 CONTINUE

WRITE $(7, *)$ 'S AVERAGE = ',SAVG,' ST.DEV. = ',STD

WRITE $(7, *)$ 'UWT = ', UWT,' UREA DIS = ',UDC, 'XUDC = ',XUDC

CALL CURVE(XP1,PR1,MMM,0)

CALL DASH

C IF (LFEED.EQ.1) GOTO 48

IF (LFEED.EQ.1) GOTO 51

LFEED $=1$

GOTO 47

48 CONTINUE

DO $49 \mathrm{JJ}=1,40$

$\operatorname{PR} 1(\mathrm{JJ})=\mathrm{JJ} / 40$

$\operatorname{READ}\left(4,{ }^{*}\right) S C 1$

$X P 1(J J)=S C 1 / 100$

C $\quad X P 2(J J)=S C 2 / 100$.

49 CONTINUE
CALL CURVE(XP1,PR1,40,-1)

C CALL MARKER(5)

C CALL CURVE(XP2,PR1,40,-1)

51 CONTINUE

CALL FRAME

CALL ENDGR(0)

CALL ENDPL(0)

C REWIND (4)

555 CONTINUE

CALL DSPDEV('PLOT')

CALL NOBRDR

CALL COMPLX

CALL AREA2D $(5.5,5$.)

CALL XNAME('Height, $m \$ ', 100)$

CALL YNAME('Coating Amount per Cycle, kg\$',100)

CALL XREVTK

CALL YREVTK

C CALL HEADIN('Density of Coating amount/CS',-100,3,1)

CALL YAXANG(0.)

IF(HCOAT.LE.0.)CALL GRAF(0,0.05,0.3,0.0,25E-4, 1.E-4)

IF(HCOAT.GT.0.)CALL GRAF(0, 0.05,0.3,0.0,.2E-6,. 1E-5)

CALL THKCRV(3)

DO 567 I = 1, N

$X P 1(I)=Z M T(1)$

$\operatorname{PR} 1(1)=$ SMT $(1)$

PRINT(2,NPRINT,I) = SMT(I)

567 CONTINUE

CALL CURVE(XP1,PR1,N,0)

CALL FRAME

CALL ENDGR(O)

CALL ENDPL(O)

RETURN

END 
SUBROUTINE PROBTR(A,B,IC)

* Initialization routine for probability plot for *disspla

ENTRY TRANS $(A, B, I C)$

CALL YPRTRN $(A, B, I C)$

RETURN

END

SUBROUTINE CUMP(M,X1,Y1,WTU)

* Sorts and calculates cumulative \#'s for plotting

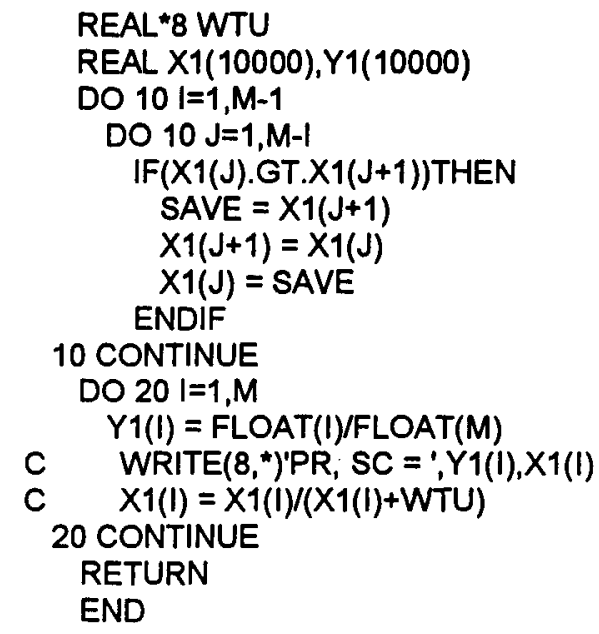




\section{SUBROUTINE PRINTO}

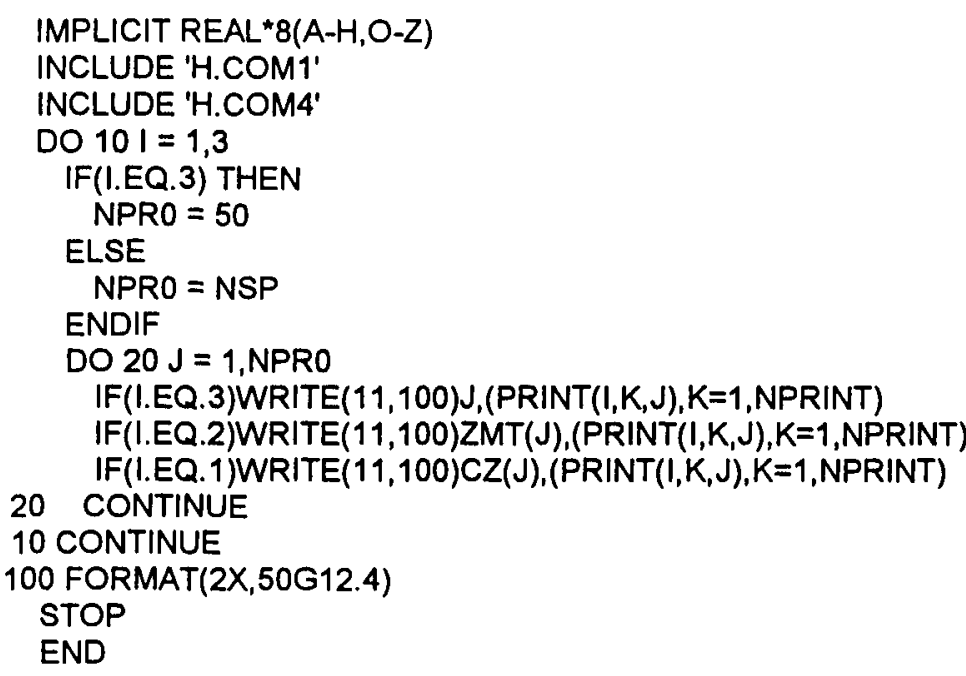

100 FORMAT(2X,50G12.4)

STOP

END

\section{DOUBLE PRECISION FUNCTION SS(X)}

* Finds the amount of sulfur a particle picks up in the spout

\section{IMPLICIT REAL*8(A-H,O-Z)}

INCLUDE 'H.COM1'

$I=\operatorname{INTT}(X, C Z, 11, D X)$

$V=\operatorname{QAINT}(X, 5)$

$E=\operatorname{QAINT}(X, 6)$

$U=$ QAINT $(X, 4)$

$C=$ QAINT $(X, 3)$

IF(V.LE.O.DO.OR.X.LT.HCOAT)THEN

$S S=0$.

RETURN

ENDIF

SS $=$ PI4*C*ETA(DABS(U-V))*DABS(U-V)*DP**2N RETURN

END 


\section{SUBROUTINE INITIA}

* This subroutine assigns the following constants to be used

* in the spouted bed hydrodynamics and coating calculations:

- $\mathrm{PI}=\mathrm{pi} \quad \mathrm{P} / 4=\mathrm{pi} / 4$

- THETA = half cone angle

- $W M A=$ molecular weight of air

- RHOSM = density of monoclinic sulfur

" RHOSR = " " rhombic sulfur

- $\mathrm{RHOU}=$ " "urea

- DPU = averaged diameter of urea

- GRAV = gravitational constant

* UNIVC = universal constant

- $\mathrm{DCOL}=$ column diameter

* $\mathrm{HB}=$ the distance from the shuttle to the bottom of the cone

- $\mathrm{DB}=$ the diameter of cone base

- TAN30 = tangent of 30 degrees

- VOID = loose packed voidage of the bed.

* NDS = dimension size for the arrays in SPLINE

- DAT = nozzle opening for air

- $\mathrm{AAT}=$ area of flow through the nozzle cap

IMPLICIT REAL"8(A-H,O-Z)

INCLUDE 'H.COM1'

NDS $=101$

$\mathrm{PI}=\mathrm{DACOS}(-1 . \mathrm{D0})$

$\mathrm{PI} 4=\mathrm{PI} / 4$.

THETA $=\mathrm{PI} / 6$.

TAN3O $=$ DTAN $($ THETA $)$

WMA $=29.0$

RHOSF $=1780$

RHOSM $=1960$.

RHOSR $=2007$.

RHOU $=1335$

$\mathrm{DPU}=0.00216$

GRAV $=9.8$

UNIVC $=.08206$

$\mathrm{DNI}=0.0029$

DAT $=0.002921$

AAT $=$ PI4*DAT $* 2$

$\mathrm{HB}=.01$

$\mathrm{DB}=.04$

Appendix III: Computer Program Listings
VOID $=0.42$

$P S \mid=1$.

\section{* Voidage calculations.}

C $\quad H=0.25+0.017$

C $\quad X S=0.31$

C $\quad W B=6.02273$

C $\quad V=C A L(9, H, H)$

C $\quad$ VP $=W B^{\star}((1 .-X S) / R H O U+X S / R H O S R)$

C VOID $=(V-V P) N$ RETURN

END 


\section{SUBROUTINE EOS(XT,DENSTY,VISCOT)}

* This subroutine calculates density and viscosity of air.

* Parameters are:

* $T=$ Temperature in Kelvin as INPUT

* $\quad D=$ Will contain density data in Kg/Cubic Metres AS OUTPUT

* $V=$ " viscosity " "Pa/s or kg.m/s AS "

IMPLICIT REAL*8(A-H,O-Z)

INCLUDE 'H.COM1'

* Density calculated from the ideal gas law. DENSTY $=1$. "WMAVUNIVCIXT

- Viscosity calculated from the correlation by Neufeld (1972) given in

* The Properties of Gases and Liquids (1977).

TSTAR $=$ XT/78.6

OMEGA $=1.16145 / \mathrm{TSTAR}^{\star *} 0.14874+0.52487^{\star} \mathrm{EXP}\left(-0.7732^{\star}{ }^{*} \mathrm{TSTAR}\right)$

$+\quad+2.16178^{\star} \operatorname{EXP}\left(-2.43787^{\star}\right.$ TSTAR $)$

VISCOT $=10.43676^{\star} X T^{\star *} .5 / O M E G A * 1 . E-7$

RETURN

END

\section{DOUBLE PRECISION FUNCTION CAL $(I, X, T)$}

* This subroutine provides calibration correlations for

* 1 = 1: Spouting air rotameter 1 (the larger of the two)

" 2: " " " 2 (the smaller " " ")

* 3: Atomizing air rotameter

* 4: Sulfur rotameter

- 5: Urea Feeder

* 6: S-type pitot tube

- 7: Smaller pitot tube

- 8: Effective shutter diameter at the spouting air inlet

* 9: Bed volume

* Parameters are

- 1 = Type of equipment as given above

* $X$ : for $I=1$ to $4, X=$ float position ( 4 - lower ball) $\wedge 5, X=$ feeder setting

$\wedge 6$ to^ $7, X=$ water manometer height difference in meters

$\wedge 8, X=$ diameter of shutter inlet, metres

$\wedge 9, X=$ bed height, metres

$T=$ density of air where the measurement was taken

$\wedge$ - dummy value of $T$ may be given for they are not used

* Units of CAL:

for I = 1 to 3 : cubic meters/s

4 to $5: \mathrm{kg} / \mathrm{s}$

6 to $7: \mathrm{m} / \mathrm{s}$

8: $\mathrm{m}$

9: cubic metres

IMPLICIT REAL ${ }^{8}(\mathrm{~A}-\mathrm{H}, \mathrm{O}-\mathrm{Z})$

IF(I.EQ.2)THEN

IF(X.LE.0.DO)THEN

$\mathrm{CAL}=0 . \mathrm{DO}$

RETURN

ENDIF

$Q=0.0194+0.0058196^{\star} X-1.12 D-5^{\star} X^{\star} X$

$+$ $+2.99820-8 * X^{* * 3}$

RETURN

ELSEIF(I.EQ.1)THEN

IF(X.LE.O.DO)THEN 
$C A L=0 . D 0$

RETURN

ENDIF

$Q=0.10089+0.0147172^{\star} X+1.55 D-5^{\star} X^{\star} X$

$\mathrm{CAL}=\mathrm{Q} / \mathrm{T} / 60$

RETURN

ELSEIF(I.EQ.3)THEN

IF(X.LE. O.DO)THEN

$\mathrm{CAL}=0 . \mathrm{DO}$

RETURN

ENDIF

C $\quad Q=(32.3-636.12 /(X-41.2)) / 1000 . / 60$.

C $\quad \mathrm{CAL}=\mathrm{Q}^{* 1.2} \mathrm{~T}$

$C A L=\left(0.811+0.2085^{\star} X\right) / 60 . / T / 1000$.

RETURN

ELSEIF(I.EQ.4)THEN

IF(X.LE. O.DO)THEN

$\mathrm{CAL}=0 . \mathrm{DO}$

RETURN

ENDIF

$C A L=\left(0.0117605^{\star} X-0.047784\right) / 60$.

RETURN

ELSEIF(I.EQ.5)THEN

$C A L=\left(.749-1.485^{\star} X+.452^{\star} X^{\star} X\right) / 60$.

RETURN

ELSEIF(1.EQ.6)THEN

CAL $=0.827^{\star}$ DSQRT $\left(2 * 9.8^{*} 998 . * / T\right)$

RETURN

ELSEIF(I.EQ.7)THEN

CAL $=1.06^{\star}$ DSQRT $\left(2^{*} 9.8^{*} 998 .^{*} \mathrm{XT}\right)$

RETURN

ELSEIF(I.EQ.8)THEN

$R=23 / 32 * 0254$

IF(X.GT.R*2.)THEN

PRINT*, ** DI BIGGER THAN POSSIBLE ***

STOP

ENDIF

$P I=D A C O S(-1 . D 0)$

$A 1=1,+D C O S(P \mid / 5$.

$A 2=1 .-D C O S(P \mid / 5 .)^{\star \star 2}$

$A=A 2+A 1^{n+2}$

Appendix III: Computer Program Listings
$B=-2 . * A 1^{*}(X-R)$

$C=(X-R)^{\star *} 2-R^{\star} R$

$X L 1=\left(-B+D S Q R T\left(B^{\star} B-4 .{ }^{\star} A^{*} C\right)\right) / 2 . / A$

$\mathrm{XL2}=\mathrm{XL1}{ }^{\star} \mathrm{DCOS}(\mathrm{PI} / 5$.)

$X L 3=D S Q R T\left(X L 1 * 2-X L 22^{\star * 2}\right)$

C

$X L 4=D S Q R T\left(R^{\star} R-X L 3^{* \star 2}\right)$

$X L 5=R-X L 4$

$A 3=5 .{ }^{*} X L 2^{*} X L 3+5 .{ }^{*}\left(D A S I N(X L 3 / R)^{\star} R^{*} R\right.$ $X$ X3*XL4) $^{*}$

$C A L=D S Q R T(4 . / P I *(A 3-.8107318 D-4))$

CAL = DSQRT(4./PI*(A3-1.*0.8107318D-4))

RETURN

ELSEIF(I.EQ.9)THEN

$\mathrm{PI} 4=\mathrm{DACOS}(-1 . \mathrm{D} 0) / 4$

$A N G=30$.

$\mathrm{DB}=0.04$

$H B=0.01$

$A B=P / 4{ }^{*} D B^{\star} D B$

$V B=H B^{*} A B$

$\mathrm{PHI}=\mathrm{ANG} / 180 .{ }^{\star} \mathrm{P} / 4^{\star} 4$

$H N=D B / 2 . / D T A N(P H I)$

$V N=H N^{*} A B / 3$

$D=0.24$

$A=P \mid 4^{*} D * D$

$\mathrm{HC}=\mathrm{D} / 2 . / \mathrm{DTAN}(\mathrm{PHI})$

$V C=A^{*} H C / 3$

$V P=V C-V N$

$\mathrm{HT}=\mathrm{HC}-\mathrm{HN}+\mathrm{HB}$

C $\quad \mathrm{VT}=\mathrm{VP}+\mathrm{VB}$

$H T=0.18320509$

$V T=0.31323016532 \mathrm{D}-2$

$A=0.4523893780696 \mathrm{D}-1$

$V A=A^{*}(X-H T)$

$C A L=V A+V T$

RETURN

ENDIF

PRINT *,$* *$ UNKNOWN CODE IN CALIBRATION SUB. ***

STOP

END 


\section{SUBROUTINE PTIN(L,N,A,B,C)}

* This subroutine finds the minimum and maximum values of arrays

- $A$ and $B$ as well as determine the axis scales of plots.

- Parameters are

$L=$ no. of divisions in axis'

$N=$ no. of data in $A$ and $B$

$A, B=$ array of $x$ and $y$ data respectively

$C=$ array containing the output values

$1,6=$ minimum $x$ - and $y$-axis values respectively

$2,7=$ length of " " " " scales

$3,8=$ maximum " " " values "

$4,9=$ minimum value in $A$ and $B$

$5,10=$ maximum " " " "

REAL $A(N), B(N), C(10)$

$A M=A(N)$

$B M=B(N)$

$A S=A(1)$

$B S=B(1)$

DO $10 I=1, N$

IF(AM.LT.A(I))AM=A(I)

IF(BM.LT.B(I))BM=B(I)

IF(BS.GT.B(I))BS=B(I)

IF(AS.GT.A(I))AS=A(I)

10 CONTINUE

$A D E L=(A M-A S) / L$

FACT $=1$.

IF(ADEL.LT.10.)THEN

$I=1$

$1000 \mathrm{FACT}=10$ **1

IF(ADEL*FACT.LT. 10)THEN

$1=1+1$

GOTO 1000

ENDIF

ELSEIF(ADEL.GE. 100)THEN

$I=-1$

2000 FACT $=10$.**1

IF(ADEL*FACT.GT. 100)THEN

$1=1-1$

GOTO 2000

ENDIF
ENDIF

$A D E L=I N T((A D E L * F A C T+5 .) / 5 .)^{*} 5 / F A C T$

$A 1=I N T(A S / A D E L) \star A D E L$

IF(A1.LT.0.)A1 = A1-ADEL

$A 3=A D E L * L+A 1$

IF(A3.LT.AM)A3=A3+ADEL

$B D E L=(B M-B S) / L$

$F A C T=1$

IF(BDEL.LT.10.)THEN

$l=1$

3000 FACT $=10^{*+1}$

IF(BDEL*FACT.LT. 10)THEN

$I=1+1$

GOTO 3000

ENDIF

ELSEIF(BDEL.GE. 100)THEN

$1=1$

4000 FACT $=1 / 110$ **

IF(BDEL*FACT.GT.100)THEN

$I=1+1$

GOTO 4000

ENDIF

ENDIF

BDEL $=$ INT((BDEL *FACT+5.)/5. $)^{\star} 5 / F A C T$

$B 1=I N T(B S / B D E L) \star B D E L$

IF(B1.LT.0.)B1 = B1-BDEL

$B 3=B D E L+L+B 1$

IF(B3.LT.BM)B3=B3+BDEL

$C(1)=A 1$

$C(2)=A D E L$

$C(3)=A 3$

$C(6)=B 1$

$C(7)=B D E L$

$C(8)=B 3$

$C(4)=A S$

$C(5)=A M$

$C(9)=B S$

$C(10)=B M$

RETURN

END

Appendix III: Computer Program Listings 


\section{SUBROUTINE MYPLO(L,M,N,A,B,XN,YN,T,C)}

* This subroutine plots data given in A and $B$. Multi plots in

* one graph are possible per call. Parameters are

- $L=$ total number of data to be plotted

- $M=$ array containing the specifications of number \& type of data to be plotted, ie. $0=$ no symbols, just a line, $1=e v e r y$ point connected by a line, $-1=$ every point not connected by a line

* $\quad N=$ array containing the number of data in the respective plots

- $A, B=x$ and $y$ data

- $X N, Y N, T=$ titles of $x$ - and $y$-axis and plot

* $\quad C=$ array containing the scales of the axis' usually determined from PTIN subroutine

REAL $A(L), B(L), C(10), X(200), Y(200)$

DIMENSION M(10),N(10)

CHARACTER $50 \times N, Y N, T$

CALL DSPDEV('PLOT')

CALL NOBRDR

CALL AREA2D $(6.0,6.0)$

CALL PHYSOR $(1,2.2)$

CALL THKCRV(0.03)

CALL XREVTK

CALL YREVTK

CALL BLSYM

CALL YAXANG(0.)

CALL XNAME $(X N, 100)$

CALL YNAME(YN,100)

CALL YTICKS(2)

CALL XTICKS(2)

CALL COMPLX

CALL HEADIN $(T, 100,-3,1)$

CALL GRAF(C(1),C(2),C(3),C(6),C(7),C(8))

C CALL GRAF(C(6),C(7),C(8),C(1),C(2),C(3))

CALL FRAME

MARK $=14$

DO $10 \mathrm{~J}=1, \mathrm{~N}(1)$

$X(J)=A(J)$

$Y(J)=B(J)$

10 CONTINUE

CALL MARKER(2)

CALL CURVE $(X, Y, N(1), M(1))$
IF(N(10).LE. 1)GOTO 200

$\mathrm{NP}=0$

DO $20 \mathrm{I}=2, \mathrm{~N}(10)$

$N P=N(l-1)+N P$

$D O 30 \mathrm{~J}=1, \mathrm{~N}(\mathrm{I})$

$X(J)=A(J+N P)$

$Y(J)=B(J+N P)$

30 CONTINUE

CALL MARKER(MARK+1)

CALL CURVE $(X, Y, N(I), M(I))$

20 CONTINUE

200 CALL ENDGR(0)

CALL ENDPL(0)

RETURN

END 
SUBROUTINE MYSPLN(X,Y,Q,R,S,N,NDS)

* Interpolation using cubic splines with fitted end points.

- Input: $X$ Array of independent $X$-values

* Y Array of dependent $y$-values

$\mathrm{N}$ Number of data points

- Output: Q,R,S Coefficients of cubic spline equations

IMPLICIT REAL*8(A-H,O-Z)

REAL"8 Y(N),X(N),H(200),A(200),B(200), C(200),D(200), TAB(200)

REAL ${ }^{\star} 8 \mathrm{Q}(\mathrm{NDS}), \mathrm{R}(\mathrm{NDS}), \mathrm{S}(\mathrm{NDS})$

* Write an error message if $N<2^{*} M$

$M=4$

IF(N.LT.M)THEN

PRINT*,'ORDER OF DIFFERENCES GREATER THAN NO OF DATA'

STOP

ELSEIF(N.LT.2*M)THEN

PRINT*,'NO OF END PTS TO BE FITTED EXCEED NO OF DATA PTS.' STOP

ENDIF

* Find coefficients A4 and B4 using divided difference method.

* The method considered here only makes use of $m$ end points on

* either end.

$\mathrm{NM}=\mathrm{N}-1$

$M P=M+1$

$M M=M-1$

$\mathrm{KIO}=\mathrm{N}-\mathrm{M}$

II $=1$

DO $10 \mathrm{I}=1, \mathrm{NM}$

IF(I.LE.MM.OR.I.GT.KIO)THEN

$T A B(I I)=(Y(I+1)-Y(I)) /(X(1+1)-X(I))$

$\|=\|+1$

ENDIF

10 CONTINUE

$10=11-M$

DO $20 \mathrm{~J}=3, \mathrm{M}$

DO $20 \mathrm{l}=1, \mathrm{MP}-$

$K M=10+1$

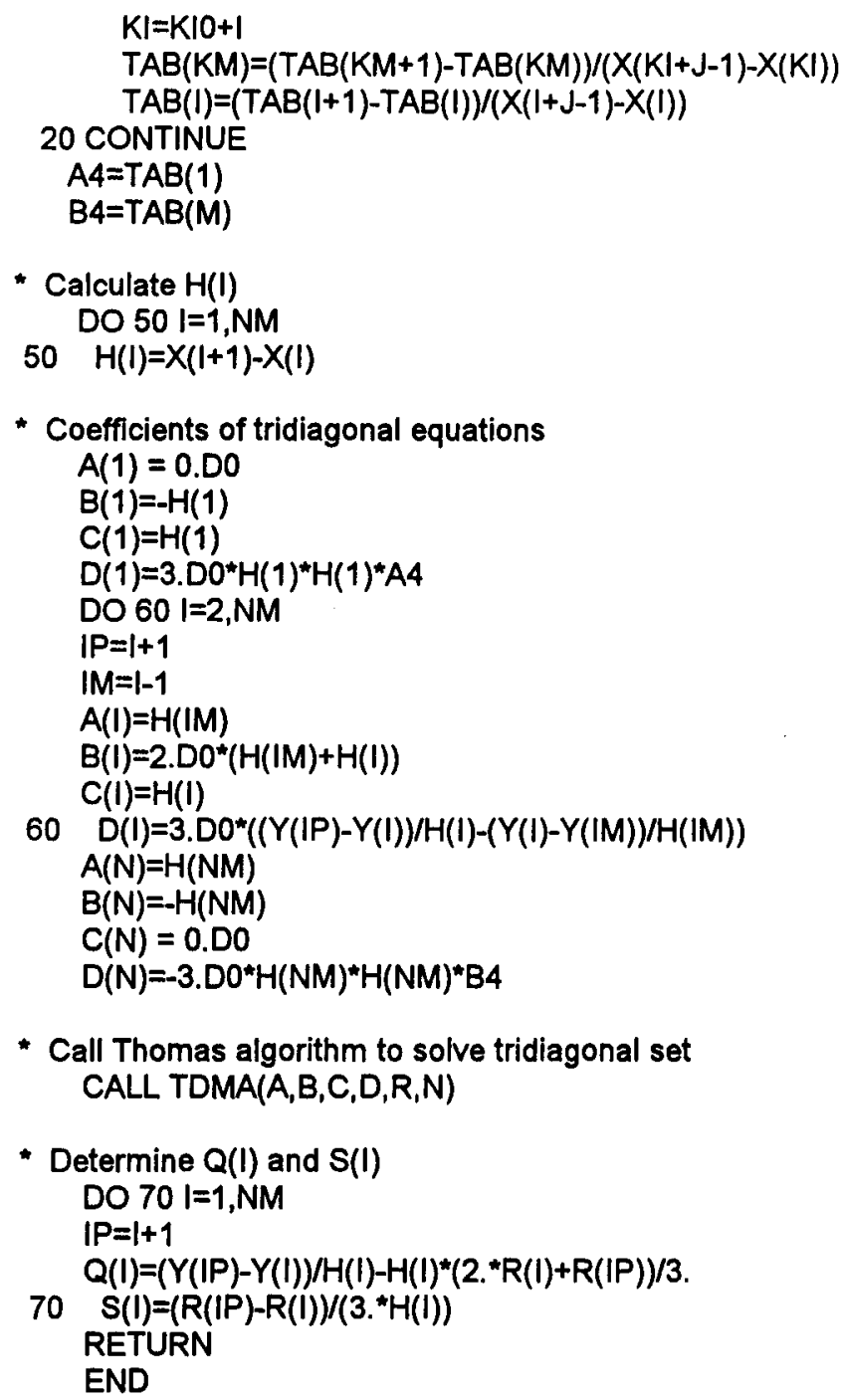

* Coefficients of tridiagonal equations

$A(1)=0.00$

$B(1)=-H(1)$

$C(1)=H(1)$

$D(1)=3 . D 0^{*} H(1)^{*} H(1)^{*} A 4$

DO $601=2, N M$

$|\mathrm{P}=|+1$

$M=1-1$

$A(I)=H(I M)$

$B(I)=2 . D 0^{*}(H(I M)+H(I))$

$\mathrm{C}(\mathrm{I})=\mathrm{H}(\mathrm{I})$

$60 D(I)=3 . D^{*}((Y(I P)-Y(I)) / H(I)-(Y(I)-Y((M)) / H(I M))$

$A(N)=H(N M)$

$B(N)=-H(N M)$

$C(N)=0 . D 0$

$D(N)=-3 . D 0^{\star} H(N M){ }^{\star} H(N M)^{\star} B 4$

- Call Thomas algorithm to solve tridiagonal set CALL TDMA(A,B,C,D,R,N)

- Determine $Q(I)$ and $S(I)$ DO $70 \mathrm{I}=1, \mathrm{NM}$ $|P=|+1$ $Q(I)=(Y(I P)-Y(I)) / H(I)-H(I) *\left(2 .{ }^{*} R(I)+R(I P)\right) / 3$.

$70 S(I)=(R(I P)-R(I)) /\left(3 .{ }^{*} H(I)\right)$ RETURN END 


\section{SUBROUTINE TDMA(A,B,C,D,X,N)}

* Thomas algorithm

IMPLICIT REAL"8(A-H,O-Z)

DIMENSION A(N),B(N),C(N),D(N),X(N),P(201), Q(201)

$\mathrm{NM}=\mathrm{N}-1$

$P(1)=-C(1) / B(1)$

$\mathrm{Q}(1)=\mathrm{D}(1) / \mathrm{B}(1)$

DO $10 \mathrm{I}=2, \mathrm{~N}$

$\mathrm{IM}=\mathrm{I}-1$

$D E N=A(I) * P(I M)+B(I)$

$P(I)=-C(I) / D E N$

$10 Q(I)=(D(I)-A(I) * Q(I M)) / D E N$

$X(N)=Q(N)$

DO $20 \|=1, N M$

$1=\mathrm{N}-\mathrm{-1}$

$20 X(1)=P(1)^{n} X(1+1)+Q(I)$

RETURN

END

\section{SUBROUTINE NC4AD(F,A,B,EPS,SUM,N)}

- This subroutine utilies the Newton-Cotes four panel method

- to integrate the function $F$ between the limits $A$ and $B$ with an

* accuracy of EPS. The integrated area is return as SUM and the

* total number of function evaluation is returned as $\mathrm{N}$.

IMPLICIT REAL $* 8(A-H, O-Z)$

DIMENSION H(20), TOL(20),SR(20),XR(20), F1(20),F2(20),F3(20)

DIMENSION F5(20),F6(20),F7(20),F8(20),F9(20),F4(20)

* Initialization: set convergence tolerance slightly lower than

"the calculated value.

IMAX $=20$

$\mathrm{N}=5$

SUM $=0 . D 0$

$\mathrm{X} 1=\mathrm{A}$

$H(1)=(B-A) / 4 . D O$

TOL(1) $=63$. DO*EPS

* Calculate the step sizes and the tolerances at each level

* up to 20 levels.

DO 10 I=2, IMAX

$\mathrm{IM}=\mid-1$

$H(I)=H(I M) / 2 . D O$

$\mathrm{TOL}(I)=\mathrm{TOL}(\mathrm{IM}) / 2 . \mathrm{DO}$

10 CONTINUE

$\mathrm{HIM}=\mathrm{H}(\mathrm{IMAX}) / 2 . \mathrm{DO}$

$X R(1)=B$

$F 1(1)=F(A)$

$F 3(1)=F(A+H(1))$

$F 5(1)=F\left(A+2 . D 0^{*} H(1)\right)$

$F 7(1)=F(B-H(1))$

$F 9(1)=F(B)$

* Calculate the total area in level 1.

$\mathrm{S}=\mathrm{H}(1) / 22.5 \mathrm{DO} 0^{*}\left(7 . \mathrm{DO} 0^{*}(\mathrm{~F} 1(1)+\mathrm{F} 9(1))+32 . \mathrm{DO}^{*}(\mathrm{~F} 3(1)+\mathrm{F} 7(1))\right.$

$+$

$1=1$

$$
\left.+12 . \mathrm{DO}{ }^{\star} \mathrm{F} 5(1)\right)
$$

$20 \mathrm{~N}=\mathrm{N}+4$

$F 2(I)=F(X 1+H(I) / 2 . D 0)$

$F 4(I)=F\left(X 1+1.5 D 0^{\star} H(I)\right.$ 
$F 6(I)=F\left(X 1+2.5 D 0^{*} H(I)\right)$

$F 8(I)=F\left(X 1+3.5 D 0^{*} H(I)\right)$

$\mathrm{SL}=\mathrm{H}(\mathrm{I}) / 45 . \mathrm{DO} 0^{*}\left(7 . \mathrm{DO}^{*}(\mathrm{~F} 1(\mathrm{I})+\mathrm{F} 5(\mathrm{I}))+32 . \mathrm{DO} 0^{*}(\mathrm{~F} 2(\mathrm{I})+\mathrm{F} 4(\mathrm{I}))\right.$

1 $\left.+12 . \mathrm{DO} 0^{*} \mathrm{~F} 3(\mathrm{I})\right)$

$\mathrm{SR}(\mathrm{I})=\mathrm{H}(\mathrm{I}) / 45 . \mathrm{DO} *\left(7 . \mathrm{DO}^{\star}(\mathrm{F} 5(\mathrm{I})+\mathrm{Fg}(\mathrm{I}))+32 . \mathrm{DO} *(\mathrm{~F} 6(\mathrm{I})+\mathrm{F} 8(\mathrm{I}))\right.$

1

$$
\left.+12.00^{\star} \mathrm{F} 7(1)\right)
$$

* If tolerance not met, divide the LHS area in two and redo

* the calculations at 20.

IF(DABS(SL+SR(I)-S).GT.TOL(I))THEN

$I M=1$

$I=1+1$

IF(I.GT.IMAX)THEN

WRITE $(8,70) \times 1$

RETURN

ENDIF

$S=S L$

$F 1(I)=F 1(I M)$

$F 3(I)=F 2(I M)$

$F 5(I)=F 3(I M)$

$F 7(I)=F 4(I M)$

$F 9(I)=F 5(I M)$

$X R(I)=X 1+4 . D 0 * H(I)$

* If the convergence criteria is satisfied, then add the area

* and restart the calculations with the next section.

$$
\text { ELSE }
$$

SUM $=S U M+S L+S R(I)$

$\mathrm{X} 1=\mathrm{X} 1+4 . \mathrm{DO} * \mathrm{H}(\mathrm{I})$

DO $40 \mathrm{~J}=1,1$

IF(DABS(X1-XR(J)).LT.HIM) GOTO 50

40 CONTINUE

$50 \quad I=J$

IF(I.EQ.1)RETURN

IM=|-1

$S=S R(I M)$

$F 1(I)=F 5(I M)$

$\mathrm{F} 3(\mathrm{I})=\mathrm{F6}$ (IM)

$F 5(I)=F 7(I M)$

$F 7(I)=F 8(I M)$

$F 9(I)=F 9(I M)$

ENDIF

Appendix III: Computer Program Listings
GOTO 20

70 FORMAT(1X,'warning - Integration Fails Beyond $X=$ ',G12.5

END 


\section{Appendix IV: \\ Calibration Results}

In this section calibration results are provided for air, sulfur and urea flowmeters, and pitot tubes used in this work (and described in Chapter 3). The rotameters for the spouting air were calibrated against standard ASME orifice meter using 1/2" and 1 1/4" orifice sizes. The rotameter for the atomizing air was calibrated against Stainless Steel Wet Test Meter (manufactured by Fisher Scientific; capacity: $680 \mathrm{~L} / \mathrm{h}$; Model Type 63115). Sulfur and urea flowmeters were calibrated from direct measurements of the flow rates using a stopwatch and weight balance. Pitot tubes were calibrated against a standard ASME pitot tube in a $0.30 \times 0.41$ by $3.7 \mathrm{~m}$ long wind tunnel attached to an Axial Flow Fan (size 15; type B-VANE; manufactured by Canadian Blower \& Forge Co. Ltd.).

The calibration results are shown in Figures IV-1 to IV-8. The solid lines in the figures represent the calibration equations as they were used in all calculations in this work. The calibration equations were derived using either linear or non-linear regressions using UBC NLP (Vaessen, 1984) available under the UBC MTSG (Runnals, 1989) main frame operating system, and are listed in Table IV-1. 
Table IV-1: Calibration equations for flowmeters and refractometer.

Figure Flowmeter

1 Spouting air rotameter 1 $Q_{s}\left(\frac{\mathrm{kg}}{\min }\right)=0.0194+0.00482 R_{R}-1.12 \times 10^{-5} R_{R}^{2}+3.00 \times 10^{-8} R_{R}^{3}$

2 Spouting air rotameter $2 Q_{s}\left(\frac{\mathrm{kg}}{\mathrm{min}}\right)=0.0101+0.0147 R_{R}-1.55 \times 10^{-5} R_{R}^{2}+8.31 \times 10^{-8} R_{R}^{3}$

3 Atomizing air rotameter $Q_{a}(\mathrm{~L} / \mathrm{min})=0.811+0.209 R_{R}$

4 Sulfur rotameter

$W_{s}(\mathrm{~kg} / \mathrm{min})=0.0118 R_{R}-0.0478$

5 Urea feeder

$Q_{s}(\mathrm{~kg} / \mathrm{min})=0.749-1.49 R_{R}+0.452 R_{R}^{2}$

$6 \quad 1 / 16 "$ pitot tube

$C_{o}($ Equation $(5.6))=1.06$

7 S-type pitot tube

$C_{o}($ Equation $(5.6))=0.827$

8 Refractometer
$C_{u}(\mathrm{~g} / \mathrm{mL})=7.20 \Delta\left(R_{I}\right)-63.5 \Delta\left(R_{I}\right)^{2}+3240 \Delta\left(R_{I}\right)^{3}$

where $\Delta\left(R_{I}\right)=R_{I}-1.3345 ; 1.3345=R_{I}$ for distilled water

$R_{R}=$ Rotameter reading or urea feeder setting; $R_{I}=$ Refractive index reading. 


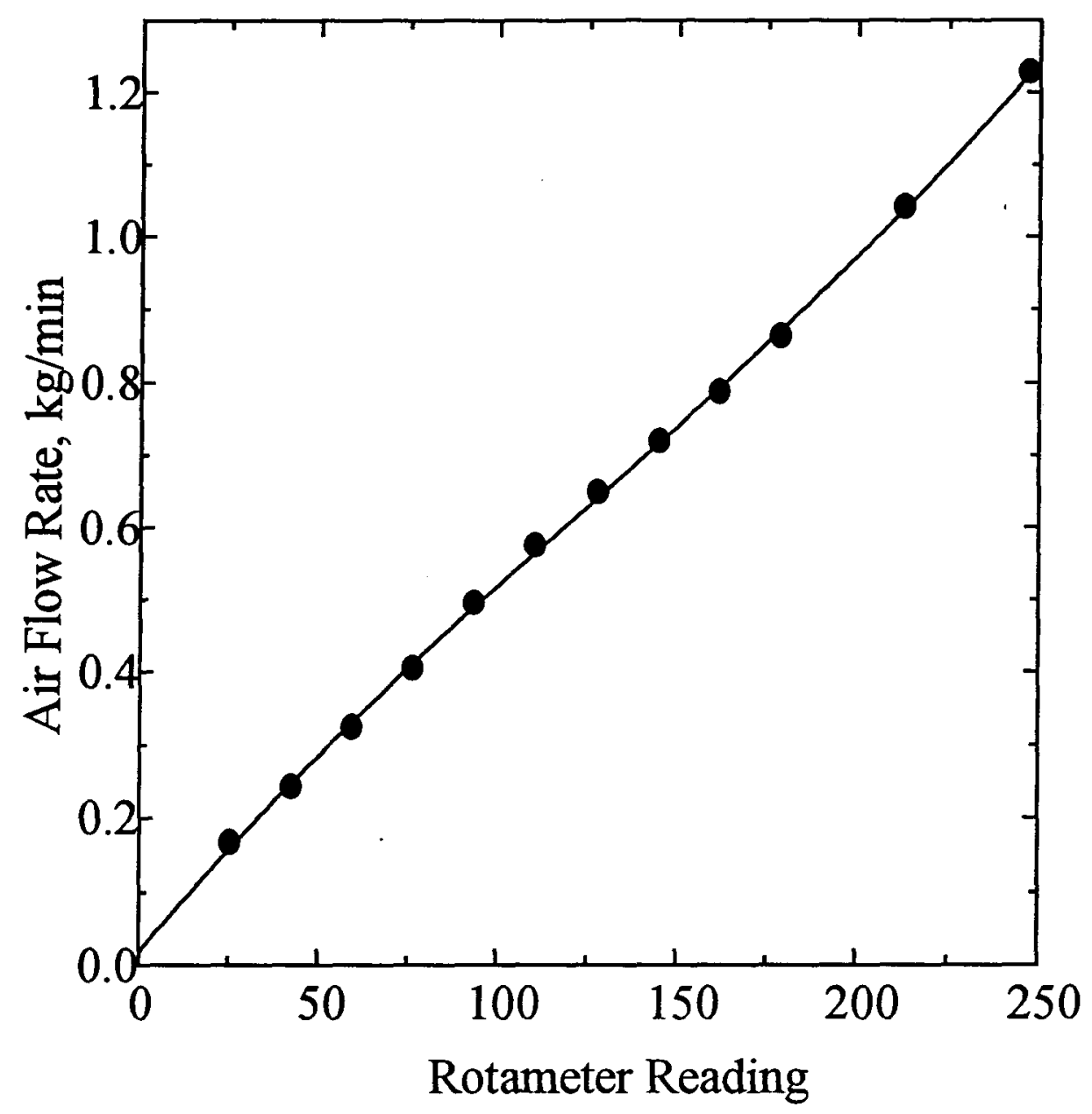

Figure IV-1: Calibration curve for the lower capacity spouting air rotameter. 


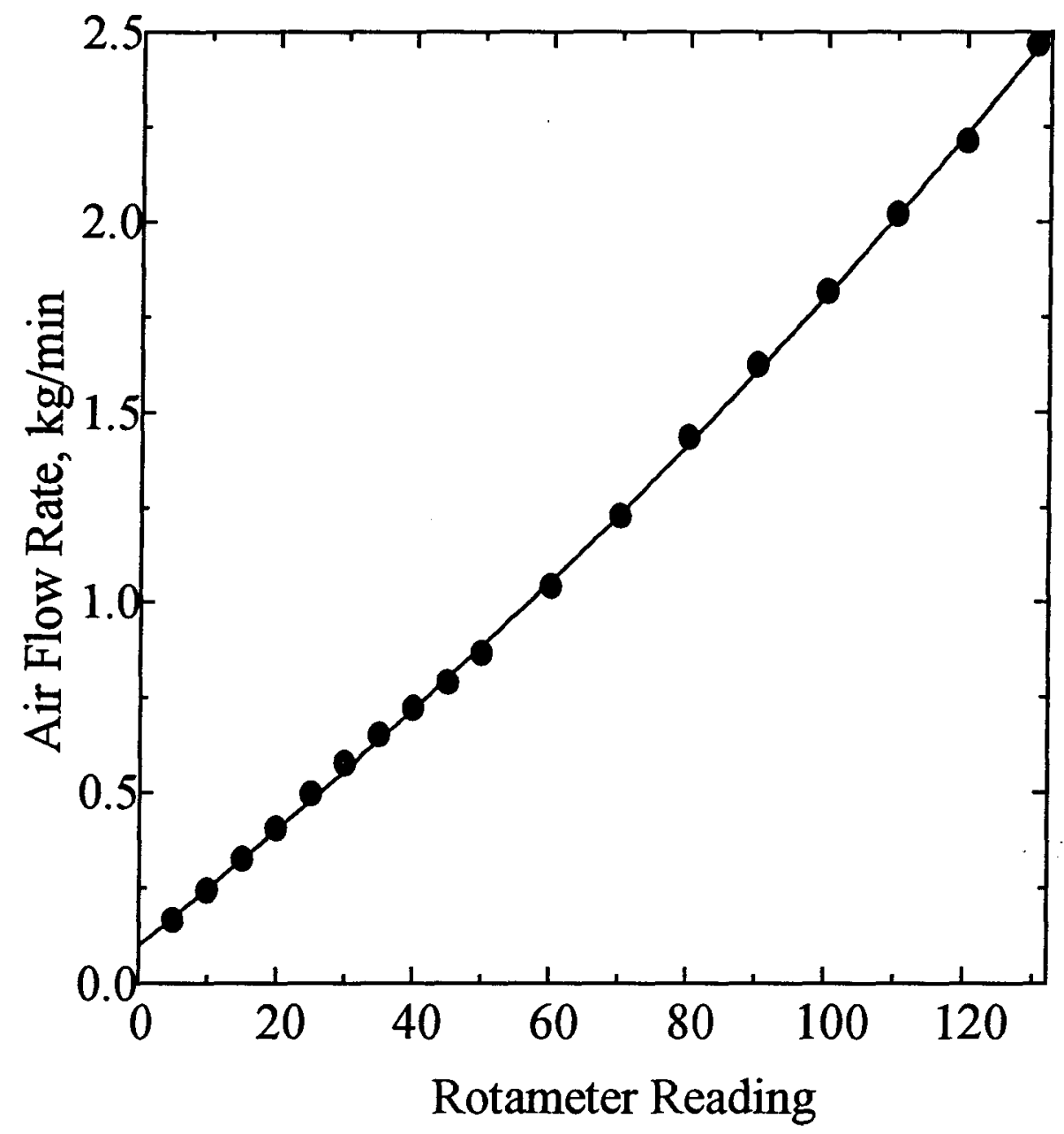

Figure IV-2: Calibration curve for the higher capacity spouting air rotameter. 


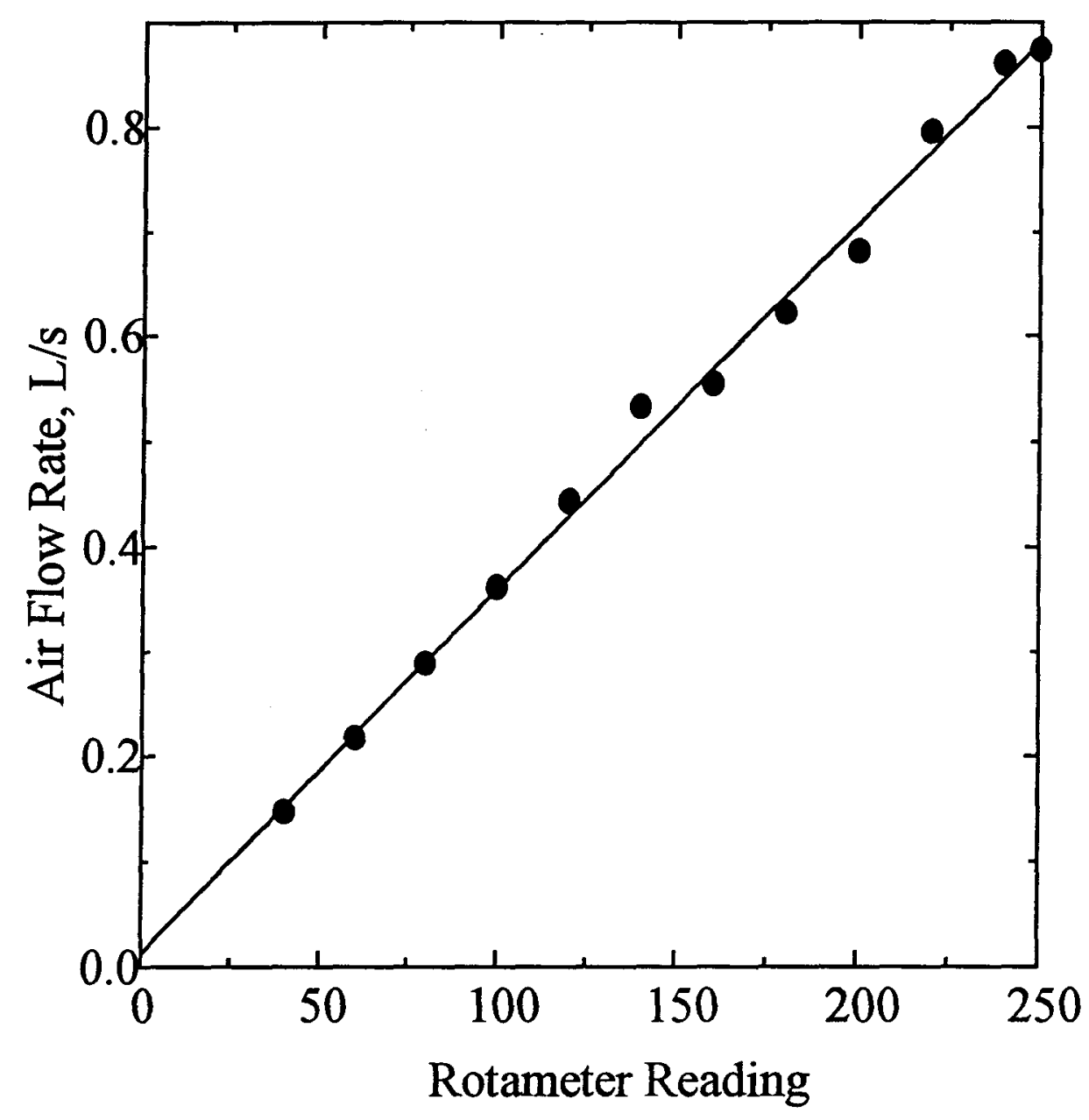

Figure IV-3: Calibration curve for the atomizing air rotameter. 


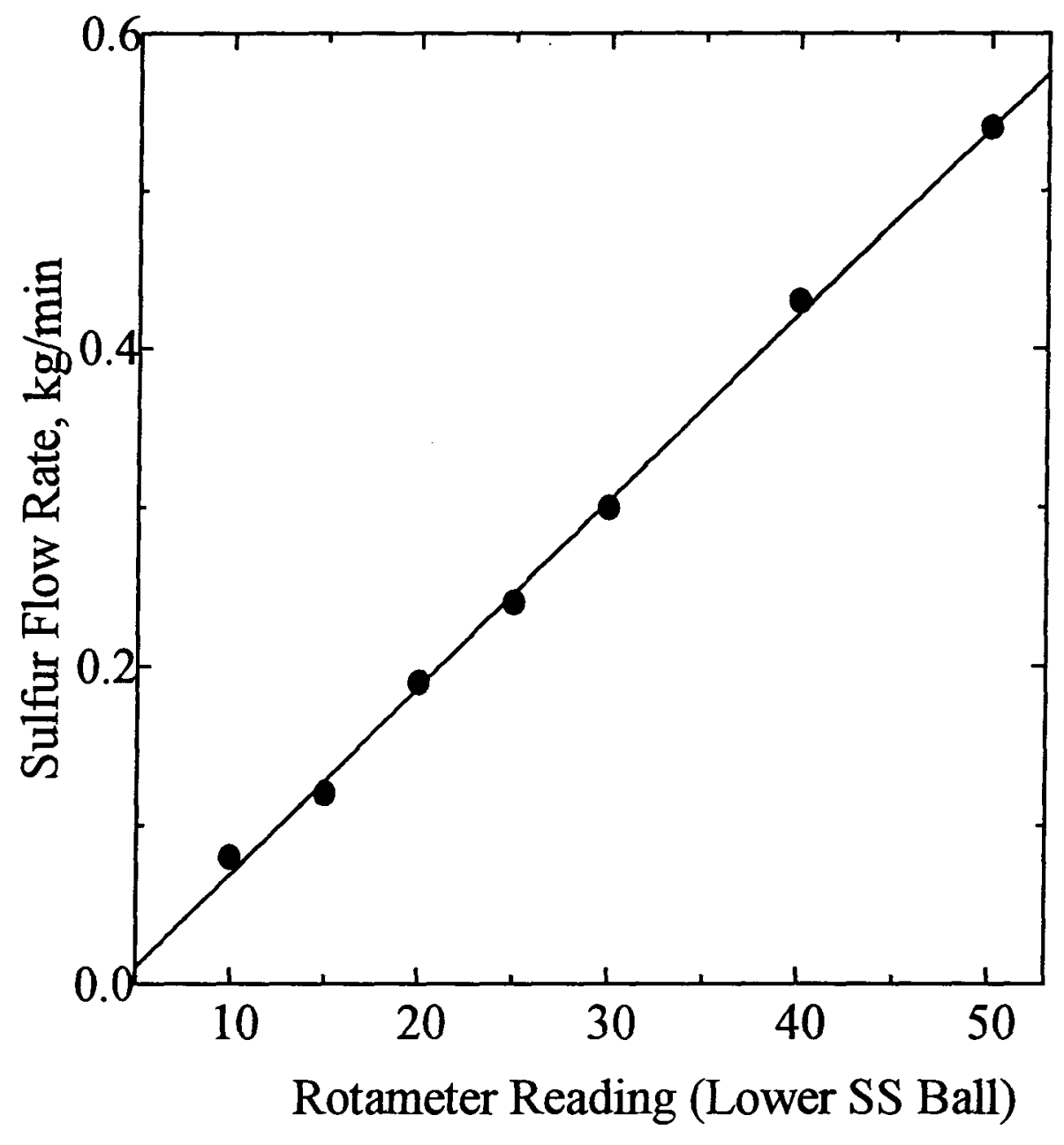

Figure IV-4: Calibration curve for the sulfur rotameter. 


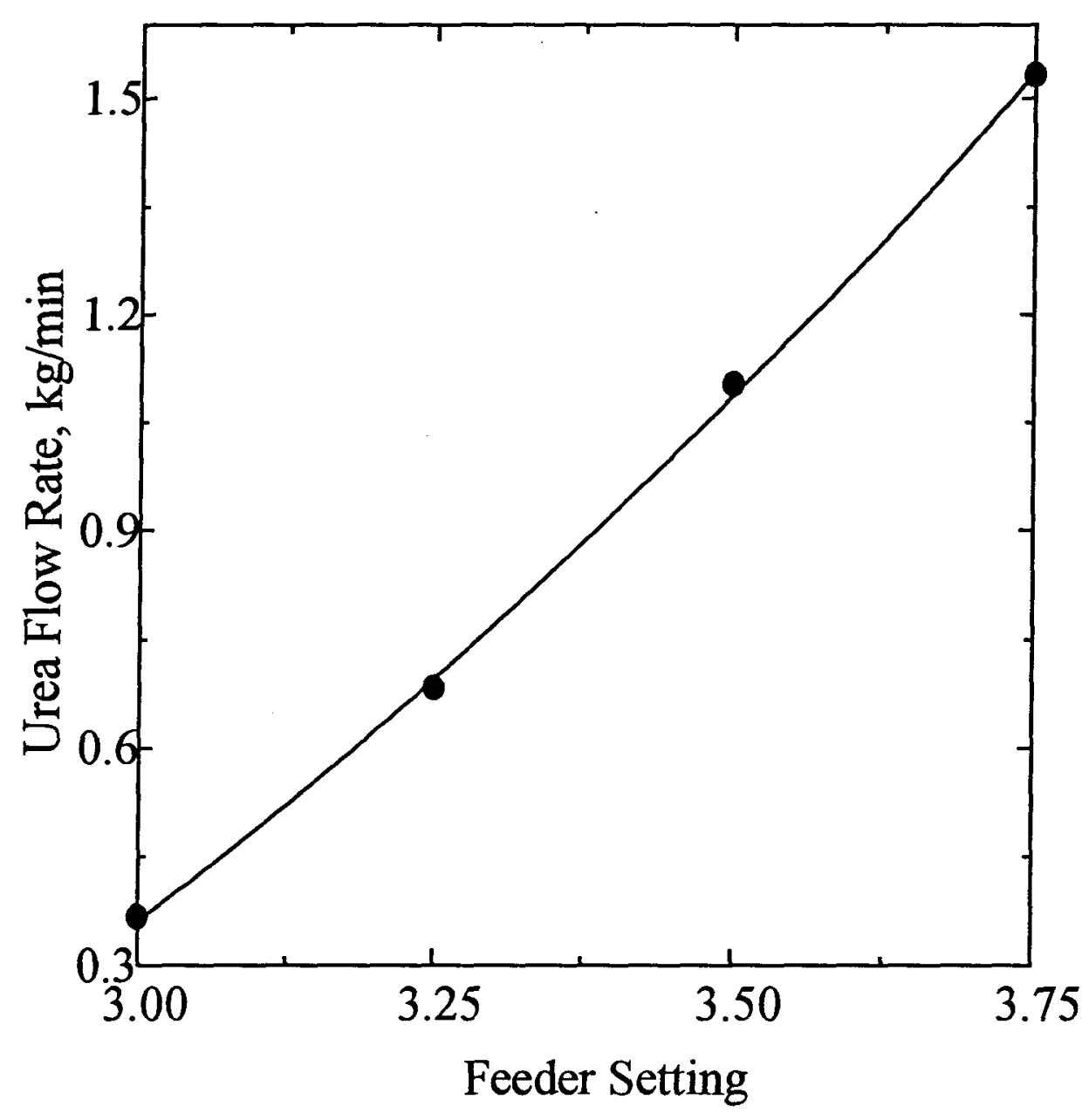

Figure IV-5: Calibration curve for the urea feeder. 


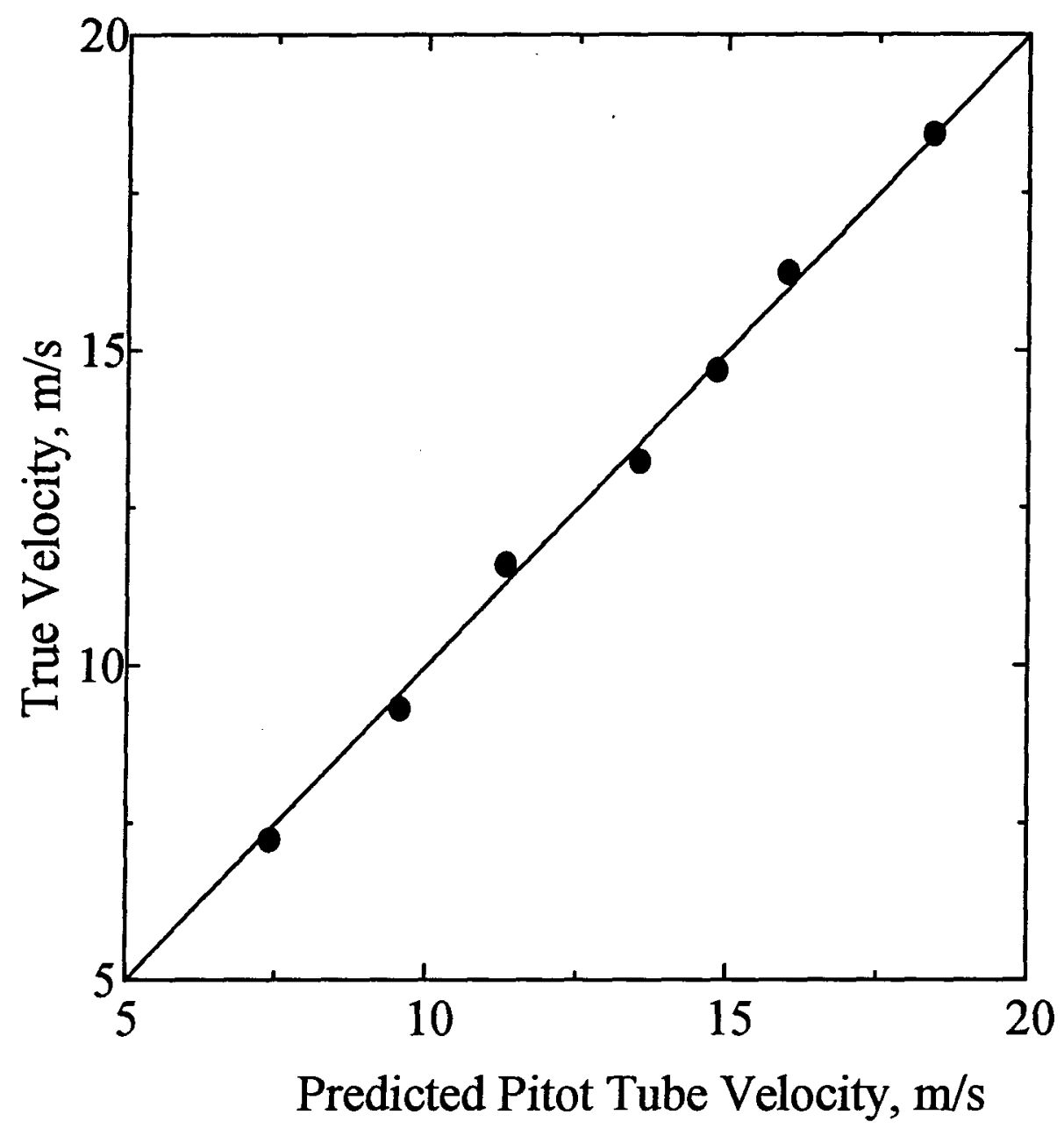

Figure IV-6: Predictions using the calibrated value of $C_{o}$ for the static-pitot tube. 


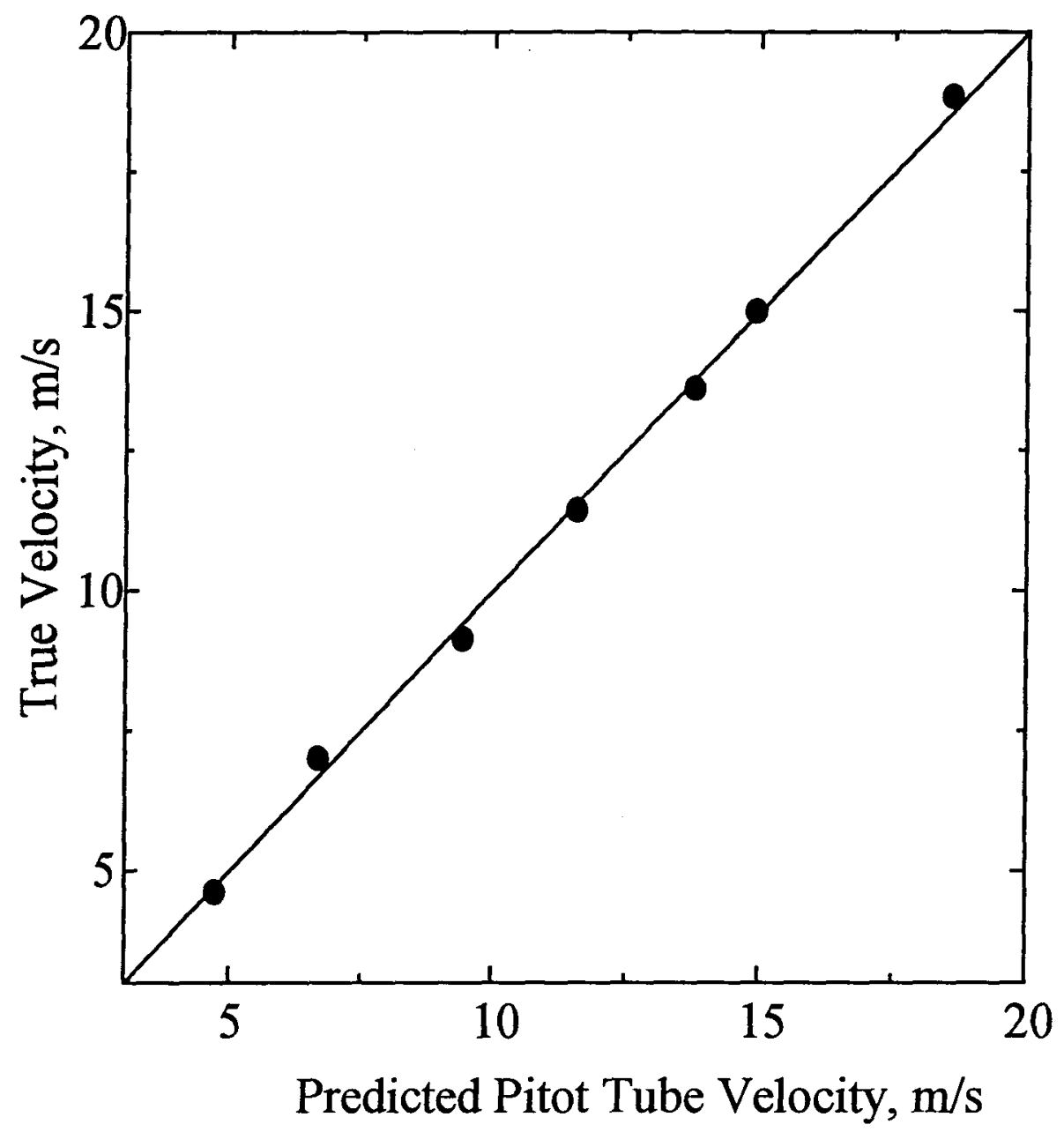

Figure IV-7: Predictions using the calibrated value of $C_{o}$ for the S-type pitot tube. 


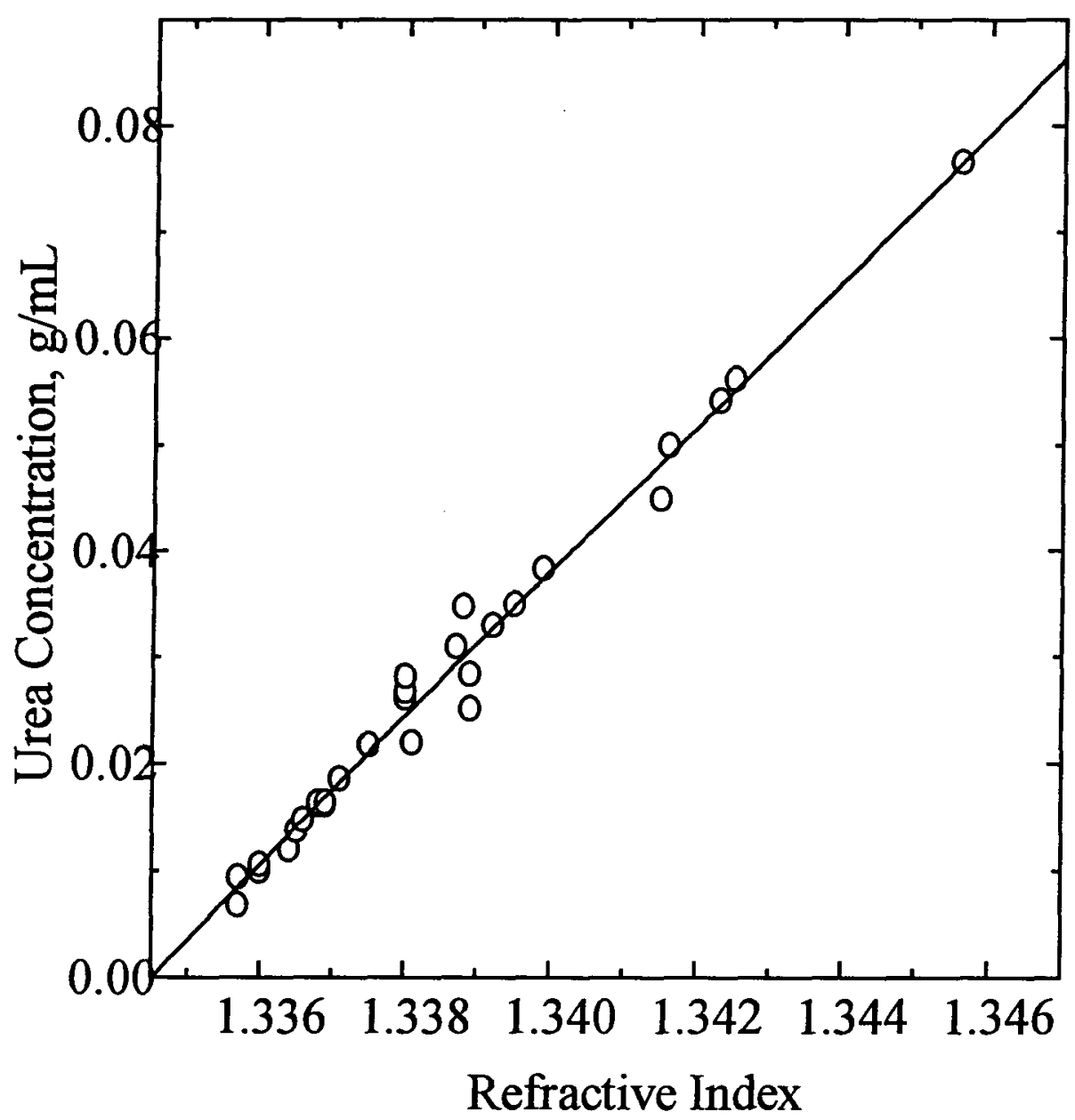

Figure IV-8: Calibration curve for the Abbey refractometer. 